\title{
ADAPTIVE CAPACITY IN ISRAELI SOCIAL CHANGE NONPROFITS
}

\author{
by \\ Nancy Jill Strichman \\ B.A. in History, University of Pennsylvania, 1992 \\ M.A. in Public Administration, Columbia University, 1996
}

Submitted to the Graduate Faculty of Education in partial fulfillment of the requirement for the degree of $\mathrm{PhD}$ in Education

\author{
University of Pittsburgh \\ 2005
}




\title{
UNIVERSITY OF PITTSBURGH SCHOOL OF EDUCATION
}

This dissertation was presented by

Nancy Jill Strichman

\author{
R. Tony Eichelberger, PhD, Professor \\ Rafael Engel, PhD, Associate Professor \\ Mary Margaret Kerr, PhD, Associate Professor \\ Fathi Marshod, M.A. \\ Dissertation Director: W. E. Bickel, PhD, Professor
}


Copyright $\odot$ by Nancy Strichman

2005 


\title{
ASSESSING NONPROFITS' ORGANIZATIONAL READINESS FOR ADAPTIVE CAPACITY
}

\author{
Nancy Jill Strichman \\ University of Pittsburgh, 2005
}

In order for nonprofit organizations to sustain themselves in today's environment, they need to continually learn from their experiences and adapt to changing circumstances. This 'adaptive capacity', considered one of the essential organizational capacities for enabling nonprofits to achieve their mission, requires nonprofits to act as 'learning organizations' and to use evaluation as a tool to enhance organizational learning and performance (Letts, Ryan \& Grossman, 1999; Sussman, 2003). A theoretical framework for assessing a nonprofit's 'organizational readiness' to improve its adaptive capacity was developed and applied to ten social change nonprofits in Israel. In this descriptive cross sectional study, a questionnaire and individual interviews with staff and board members were used to gain insight into the nonprofits' work environments and learning cultures. The results demonstrate the utility of the conceptual framework while providing insight into the dayto-day realities of organizational life that help to shape the adaptive capacity of the ten nonprofits in the sample. Practical implications can be drawn regarding the development of capacity building strategies and possible focal points for the strengthening of adaptive capacity among social change nonprofits. 


\section{ACKNOWLEDGEMENTS}

I would first like to express my gratitude to my dissertation chair and advisor, Professor William Bickel. From his early encouragement to apply for a $\mathrm{PhD}$ to his unwavering support throughout the three year process, Professor Bickel was always there guiding me along with important ideas and practical wisdom. I feel incredibly fortunate to have had my dissertation experience shaped to such a great extent by his significant contribution and constant enthusiasm for the work.

Special thanks are also due to Fathi Marshood, my committee member and mentor in Israel, who has enriched the study in every way. He provided invaluable help in determining my research sample and by offering his vast expertise in the field of nonprofits in Israel. His optimism and faith in the power of nonprofits to bring about social change during difficult times in Israel served as a constant source of motivation for me.

I would also like to extend my sincere appreciation to the other members of my committee, Professor R. Tony Eichelberger, Dr. Rafael Engel, and Dr. Mary Margaret Kerr for their keen interest and wholehearted support of the study. My committee provided me with meaningful insights and provocative questions throughout the process. I am also lucky to have been able to share this journey with my research colleague, Judith Toure, who has cheered me on since our first day in Core class together.

I wish to acknowledge the nonprofits operating in Israel that agreed to participate in the study. The staff members of these nonprofits are truly inspiring, and I will always be indebted to them for opening themselves up and allowing me inside their organizations.

I certainly would not have made it through such as process without the support of my wonderful family. To my siblings, Debbie, Andrea and Jeff, I want to thank for their countless words of encouragement and for always helping me to keep things in perspective (and with my sense of humor intact). I am, of course, especially grateful to my parents and grandparents, who always 
placed such a high priority on education and encouraged me throughout the many stages in my life that ultimately led me to pursue a $\mathrm{PhD}$. I can especially thank the first Dr. Mom in the family who is not only my best editor and cheerleader but the most significant role model that I could be lucky enough to have. I know how proud she and my Mama (who just celebrated her $95^{\text {th }}$ birthday) are of me, and I know that Papa would have been too. Lastly, I want to thank my adorable family: my dear Ofer, who supported me in every way imaginable, and my two little ones, Yonatan and Nomi, who are my everything. 


\section{TABLE OF CONTENTS}

1.0 INTRODUCTION AND CONTEXT OF THE STUDY

1.1 BACKGROUND__ 1

1.2 PROBLEM STATEMENT__ 7

1.3 OVERVIEW OF THE STUDY__ 9

2.0 LITERATURE REVIEW

2.1 LEARNING ORGANIZATIONS 12

2.1.1 Exploring Transformational Learning ___ 13

2.1.2 The "Learning System" of a Learning Organization ___ 16

2.1.3 Summarizing a Theoretical Framework for a Learning Organization___ 36

2.2 EVALUATION IN ORGANIZATIONS 37

2.2.1 Evaluation and Organizational Learning ___ 37

2.2.2 Evaluative Inquiry as Participatory Evaluation __ 39

2.3 PRACTICAL METHODS OF ASSESSMENT _ _ 47

2.3.1 Litmus Tests__ 48

2.3.2 Assessment Tools __ 51

2.4 THE NONPROFIT CONTEXT _ 61

2.4.1 The Nonprofit Sector in Israel ___ 62

2.4.2 Culture of Nonprofits___ 64

2.4.3 Culture of Philanthropy ___ 65

2.4.4 Capacity Building for Nonprofit Organizations ___ 67

2.4.5 Adaptive Capacity___ 69

2.4.6 Organizational Readiness___ 72

2.5 IMPLICATIONS FOR THE STUDY__ 75

3.0 METHODOLOGY AND DATA ANALYSIS _ 79

3.1 CONTEXT OF THE STUDY _ 79

3.2 RESEARCH QUESTIONS AND RATIONALE___ 80

3.3. SITE SELECTION — 82

3.4 METHOD _ 84

3.6 DATA ANALYSIS TECHNIQUES _ 89

3.7 PILOT TEST__ 91

3.8 LIMITATIONS AND STRENGTHS OF THE STUDY __ 92

3.9 PRESENTATION OF THE RESEARCH FINDINGS _ 93

4.0 RESULTS _ _ 95

4.1 SHARED VISION — 96

4.2 INQUISITIVENESS/OPENNESS__ 106

4.3 SYSTEMS/ EVALUATIVE THINKING__ 117

4.4 SOCIAL CAPITAL _ 127

4.5 NETWORK/EXTERNAL CONNECTEDNESS ___ 138 
5.1 REVIEW AND REFLECTIONS ON THE STUDY 147

5.2 SUMMARY OF THE RESEARCH 148

5.3 DIRECTIONS FOR FUTURE RESEARCH 152

5.4 IMPLICATIONS FOR PRACTICE 154

APPENDICES

APPENDIX A: ASSESSMENT QUESTIONNAIRE FOR NONPROFITS 159 APPENDIX B: DEVELOPMENT OF ASSESSMENT QUESTIONNAIRE 164 APPENDIX C: INTERVIEW GUIDE FOR STAFF AND BOARD 167 APPENDIX D: FRAMEWORK FOR ASSESSMENT OF ADAPTIVE CAPACITY 168 APPENDIX E: CODING CHART OF QUALITATIVE DATA AND SAMPLE INTERVEWS APPENDIX F: PILOT QUESTIONNAIRE APPENDIX G: QUESTIONAIRE RESULTS BY ORGANIZATION 170 APPENDIX H: DESCRIPTIVE STATISTICS BY ORGANIZATION 174 177 


\section{LIST OF TABLES}

Table 1: Effective Communities of Practice (Preskill \& Torres, 2000a, p. 29) ___ 28

Table 2: Informal Learning in the Workplace (Volpe \& Marsick, 1999) ___ 32

Table 3: A Theoretical Framework for a "Learning Organization" ___ 36

Table 4: Dimensions of the Learning Organization Questionnaire (DLOQ)

Table 5: Readiness for Organizational Learning and Evaluation Instrument (ROLE)__ 56

Table 6: Organizational 'Learning Capacity' Assessment ___ 58

Table 7: Traditional Funding Approach to Nonprofits___ 66

Table 8: Four Dimensions of Adaptive Capacity (Sussman, 2004) _ 70

Table 9: Assessing Learning Culture Scale Items (Botcheva, White \& Huffman, 2002) ___ 74

Table 10: Understanding Organizational Readiness for Adaptive Capacity Building__ 77

Table 11: Background Information on the Ten Participating Nonprofits___ 83

Table 12: Data Collected from the Participating Organizations ___ 88

Table 13 : Aspects of Adaptive Capacity for the Participating Nonprofits 146

Table 14: Potential Pitfalls and Potential Remedies for Enhancing Adaptive Capacity __ 156 


\section{LIST OF FIGURES}

Figure 1: Preconditions for Influencing Social Change 7

Figure 2: Barriers to Influencing Social Change 8

Figure 3: "The Whole is Greater than the Sum of Its Parts" (Sweeney, 2001) 23

Figure 4: Illustrations of Cause and Effect (Sweeney, 2001) 24

Figure 5: Review of Barriers to Influencing Social Change 76 


\subsection{INTRODUCTION AND CONTEXT OF THE STUDY}

\subsection{BACKGROUND}

The sustainability of nonprofit organizations represents one of the most pressing issues currently facing the public sector (Wolf, 1999). ${ }^{1}$ Rising costs, technological changes, greater competition among providers, increasingly diverse constituents and higher expectations from the public and funders are significant factors to which nonprofits respond in today's environment (Wolf, 1999). To remain viable, nonprofits, like all organizations, need to continually adapt themselves to an environment characterized by rapid changes. In recent years, we read a great deal about the impact of trends such as globalization, advanced communication, and technology on our society. Just as individuals and communities must learn to cope with today's fast-paced reality, organizations must also continually assess their changing environment. Because "we basically do not know what the world of tomorrow will really be like except that it will be different," organizations have to become "perpetual learners" (Schein, 1992, p. 361). Nonprofits, currently suffering from a "crisis of confidence," are under particular pressure to demonstrate their organizational effectiveness (Wolf, 1999, p. 2) and to maximize their social impact.

In recent years, organizational capacity building efforts for nonprofits have been the subject of considerable debate and significant activity (e.g., Light et al., 2004). Capacity building efforts are broadly defined by Light et al. (2004) as activities "designed to improve the performance of an organization by strengthening its leadership, management, or administration" (p. 13). Adaptive capacity, which requires nonprofits to act as learning organizations and to use evaluation as a tool to enhance organizational learning and performance (Letts, Ryan \& Grossman 1999; Sussman, 2003), is considered the most critical dimension of organizational capacity (Connolly \& York, 2003). This study explores the 'organizational readiness' of social change nonprofits in Israel to improve their adaptive capacity.

\footnotetext{
${ }^{1}$ Wolf (1999) is referring specifically to nonprofits operating in the United States. While keeping in mind the very distinctive political and social circumstances of Israel, it is possible to generally apply the overall contextual framework of American nonprofits and the relevant literature to Israeli nonprofits (See Subsection 2.4.1).
} 
In this chapter, selected concepts from the research on capacity building, organizational learning and evaluation that together contribute to the overall conceptualization of adaptive capacity are introduced. First, a short discussion on organizational capacity building sets the stage for the study and highlights the unique context of the nonprofit organization. Aspects of adaptive capacity, particularly two critical elements of adaptive capacity, the creation of work environments that are conducive to learning ('learning organizations'), and the practical use of evaluation, are discussed. Following a brief review of these key concepts, the research problem and the context of the study are addressed.

\subsubsection{Organizational Capacity Building}

In an environment that is characterized by both growing needs and shrinking resources, nonprofits around the world are challenged to effectively confront increasingly complex social issues and create social value. Traditionally, organizational effectiveness for nonprofits has been measured by a vital mission, an active board of directors, capable staff and volunteers and stable finances (Heuer, 1999). Today high performing nonprofits need to also be responsive, innovative, and act as 'learning organizations' (Letts, Ryan \& Grossman, 1999; Heuer, 1999). What is necessary, according to Letts, Ryan and Grossman (1999), is to redefine the challenge for nonprofits, shifting the focus from the implementation of successful programs to the building of effective organizations. As the authors explain, "the missing ingredient in the prevalent, program-centered conception of social impact is organizational capacity" (Letts, Ryan \& Grossman, p. 3). In recent years, there is greater awareness of the importance in investing in this "missing ingredient."

While there are a variety of types of capacity building (Blumenthal, 2003), the general approach by nonprofits and funders represents a willingness to invest in the basic operating functions of the organization and ultimately enhance the long term stability of the organization. Capacity building has been described in detail as "the development of an organization's core skills and capabilities, such as leadership, management, finance and fundraising, programs and evaluation, in order to build the organization's effectiveness and sustainability" (Campobasso \& 
Davis, 2001, p.1). ${ }^{2}$ Capacity building efforts, designed to strengthen organizations at any stage of development, can be of value to those established organizations seeking to improve their performance as well as to new organizations hoping to get beyond "basic survival" (Campobasso \& Davis, 2001, p.1).

Basic survival, or sustainability, considered as one of the greatest challenges facing nonprofits today, depends on its organization's ability to anticipate change and to adapt to varying circumstances (Wolf, 1999). ${ }^{3}$ In the nonprofit sector, this essential organizational capacity for enabling nonprofits to achieve social impact has been described as 'adaptive capacity' (Letts, Ryan \& Grossman, 1999). It is adaptive capacity that supports learning, innovativeness and responsiveness, and ensures that the nonprofit is fulfilling its organizational mission; "For an organization to be more than the sum of its programs, it needs the ability to ask, listen, reflect and adapt" (Letts, Ryan \& Grossman, 1999, p. 21). Among the four core organizational capacities that are considered critical for nonprofits: adaptive capacity, leadership capacity, management capacity and technical capacity, adaptive capacity is considered as the most vital (Connolly \& York, 2003). Adaptive capacity requires nonprofits to act as learning organizations and to use evaluation as a tool to enhance organizational learning and performance (Letts, Ryan \& Grossman 1999; Sussman, 2003). Both of these important aspects of adaptive capacity are explored briefly in the following two sections.

\subsubsection{Learning Organizations}

For any type of organization, the capacity to learn is considered as crucial for ensuring long term organizational stability and productivity (Garvin, 2000; Argyris \& Schon, 1996; Senge, 1990). The term 'learning organization', popularized by Senge in the early 1990's, has become part of the everyday vocabulary in the management literature. The theoretical framework of a learning organization, outlined in Chapter Two, draws from the field of organizational learning, as well as from theories of social capital, knowledge management and informal learning. While there is no

\footnotetext{
${ }^{2}$ For more information about various approaches to capacity building, also see Draper, 2000, Blumenthal, 2003.

${ }^{3}$ There are varying definitions of sustainability. Wolf (1999), for example cites the importance of an organization's ability to be sustainable is relevant to its constituent's needs, accountable, managed effectively and financially sound, while placing the ability to adapt to change as the main indicator for sustainability. David (2002) discusses the sprit, values, niche and capacity of an organization as the essential ingredients for sustainability.
} 
universally accepted definition of a learning organization, there is general agreement that organizational learning is a long-term, extensive process that incorporates the acquisition and application of new knowledge and insights. Bickel, Millet and Nelson (2002) offer a comprehensive definition of the concept:

A learning organization at its core is one that evidences a commitment to inquiry, exhibits fluid information exchange across organizational boundaries (external and internal), possesses knowledge management systems that facilitate collective learning, and demonstrates strategic as well as tactical decision-making based upon what is being learned (p. 1).

Organizations can learn only when individuals learn (Senge, 1990, Preskill \& Torres, 1999; Garvin, 2000), and a critical element in learning organizations is the kind of environment that can successfully foster these types of processes and practices. Volpe and Marsick (1999) summarize factors that enhance learning in the workplace:

People learn more when they continually scan their environment, heighten their awareness around learning, pay attention to goals and turning points, and develop skills of reflection while taking action. Finally, people learn more easily in a culture of collaboration and trust (p. 1).

Most organizations would, of course, like to consider themselves as successful in creating an organizational environment that enhances their ability to act as 'learning organizations'. Yet it is often the case that learning organizations are "embraced in theory but are surprisingly rare" in practice (Garvin, 2000, p. ix). Why is it so difficult for an organization to foster an environment that is conducive to becoming a learning organization? Perhaps it is because the concepts of a learning organization are considered too abstract (Davenport \& Prusak, 1998) and difficult to translate into action (Garvin, 2000). Or perhaps the challenges of organizational change are continually underestimated and the expectations for immediate results are too high. Indeed, as Ziegler (1999) reports, "the learning process is far more ambiguous, messy and elusive in reality than is depicted in the popular management literature" (p. 62).

It is widely acknowledged in the literature that the ability to become a learning organization is significantly affected and shaped by the organization's culture that can either inhibit or facilitate learning (Schein, 1992). It is possible to consider if organizations tend to minimize the complexity of cultural transformation and embark upon organizational change efforts without first understanding the culture that they seek to change. Are the current obstacles to learning 
acknowledged? Or, alternatively, are the positive aspects of the organization's culture and values that should be reinforced systematically identified? An essential step towards becoming a learning organization is to assess the current condition of the organization and its readiness for organizational learning (Marsick and Watkins, 2003; Preskill \& Torres, 2000b; Davidson, 2001). Furthermore, ongoing assessment of the organization's culture and work environment can also serve as a guide to change and allow the organization to continually measure its progress. It is important to acknowledge that becoming a learning organization is not a goal that can be achieved so we can check it off the "to do" list, but rather a "way of organizational life" (Raelin, 2000, p. 28), a "state of being" (Ziegler, 1999, p. 65).

\subsubsection{Evaluation as Learning}

The process of evaluation, generally conceptualized as the gathering of information to inform decision-making, is the second critical component in ensuring that an organization has adaptive capacity. Whereas traditional evaluation methods have often created the impression of a 'report card' mentality, recent approaches are pushing evaluation beyond its original boundaries of primarily being used to judge merit and worth (Gray, 1998, p. xv). Increasingly, participatory models of evaluation are being adopted by organizations in an effort to empower its staff, create multiple learning opportunities and promote organizational development (Patton, 1998; Preskill \& Torres, 1999, 2000a; Cousins \& Earl, 1995).

A brief description of a participatory evaluation model (Patton, 1998) clarifies how evaluation to enhance learning and organizational performance can be distinguished from more traditional approaches to evaluation. ${ }^{4}$

1. Evaluation helps to promote organizational effectiveness.

2. Evaluation represents an ongoing, continuous process.

3. Evaluation is designed to enhance learning.

4. Evaluation is carried out by organizational members.

5. Evaluation is accessible and "doable". (Patton, 1998)

\footnotetext{
${ }^{4}$ Patton (1998) is referring specifically to 'co-evaluation'. Various models of participatory evaluation will be further discussed in depth in Chapter Two.
} 
Evaluation to support ongoing learning focuses on organizational effectiveness, with an emphasis on the assessment of the entire organization versus the assessment of a particular project or program. A second distinguishing feature is that evaluation is ongoing. Whereas traditional evaluation is episodic and designed to provide summative reports at the end of the project, evaluation to support organizational learning is formative in its nature and integrated in the everyday activities of the organization. ${ }^{5}$ A third attribute of participatory evaluation is the promotion of evaluation as learning with an emphasis on how to improve rather than making a summative judgment of merit or worth. The fourth characteristic of participatory evaluation is evaluation as internal. Rather than being determined by external demands for accountability, "the new vision of evaluation is that the highest form of accountability is self-accountability" (Patton, p. xii). Last, the view of evaluation as doable departs from the traditional social science approaches that have made evaluation "inaccessible, academic and often irrelevant" (Patton, p. xiii).

In Chapter Two, participatory models of evaluation are explored, with a particular focus on the role of evaluative inquiry in organizations and its emphasis on factors such as collaborative learning, linkage between learning and performance, training in inquiry skills, and solicitation of diverse perspectives (Preskill \& Torres, 1999, p. xx). By their very nature, participatory models, such as evaluative inquiry, necessitate the full involvement and engagement of the organizational members. It is, therefore, vital to ensure that the organization itself is prepared to engage in the process of evaluation that ultimately leads to new insights and inevitable change. Understanding the 'infrastructure' and the reasons that the evaluation is being carried out is essential (Preskill \& Torres, 1999; Chelimsky, 2001). This is critical because "organizations are political entities with individual cultures built upon their past histories and (that) they often possess a self-protective infrastructure that cushions them splendidly against demands for change" (Chelimsky, p. 17).

\footnotetext{
${ }^{5}$ Summative evaluation provides a "definitive judgments about effectiveness" whereas formative evaluation focuses on "ways of improving and enhancing programs" (Patton, 1997, p. 67). In explaining the difference, Patton cites Stake (1991): "When the cook tastes the soup, that's formative, when the guests taste the soup, that's summative" (p. 69, quoted from Scriven, 1991).
} 


\subsection{PROBLEM STATEMENT}

How is possible to ensure that efforts to improve overall organizational capacity help a nonprofit organization to develop a "generalized coping mechanism" to deal with change, unpredictability and instability (Garvin, 2000, p. 126)? This "generalized coping mechanism" for an organization has been described as 'adaptive capacity' in the nonprofit sector (Letts, Ryan \& Grossman, 1999). It is adaptive capacity that supports evaluation practices and organizational learning, and ensures that the nonprofit is fulfilling its organizational mission. Figure 1 helps to place adaptive capacity in the overall context of nonprofit organizational capacity building.

Figure 1: Preconditions for Influencing Social Change

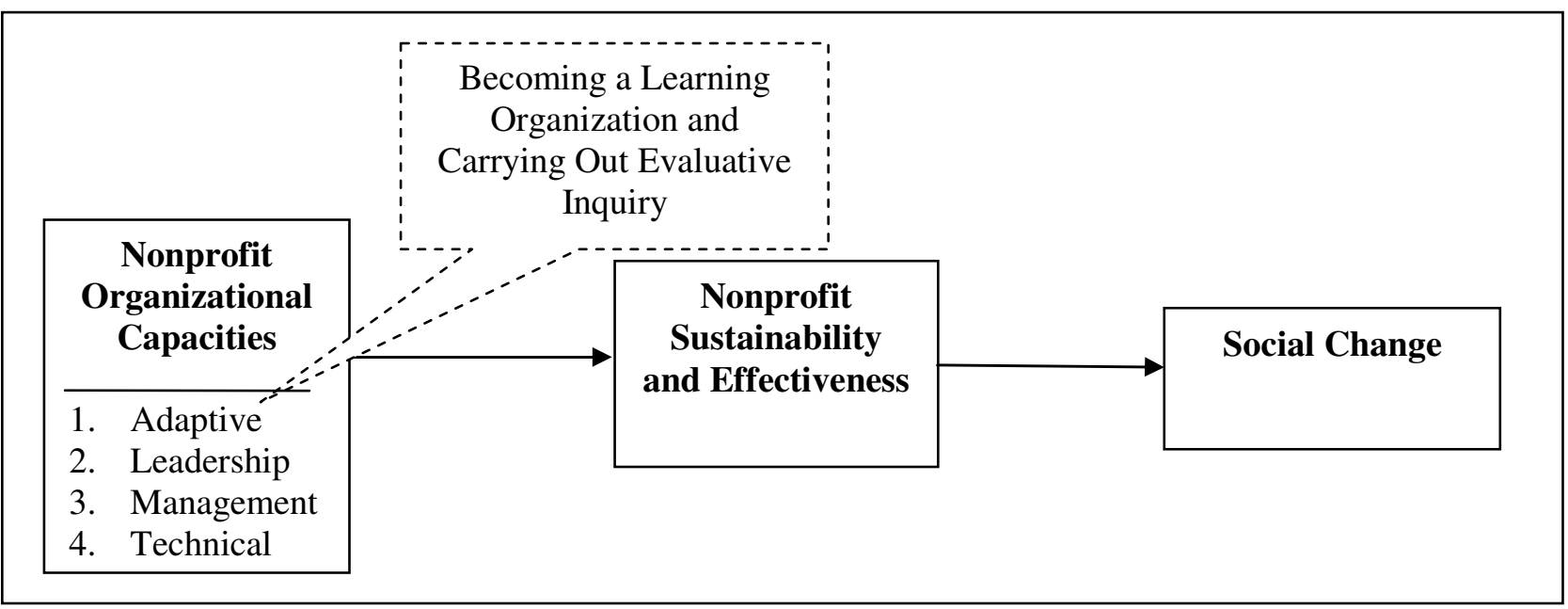

As illustrated in Figure 1, the ultimate goal of the nonprofits is to create social value and bring about positive social change. In order to maximize their social impact, nonprofits need to be able to sustain themselves and continually enhance their organizational performance and effectiveness. To do so, nonprofits should have four core capacities: adaptive capacity, leadership capacity, management capacity and technical capacity (Connolly \& York, 2003). While each organizational capacity plays a vital role in ensuring long term success for 
nonprofits, adaptive capacity is considered as the most important dimension of organizational capacity (Connolly \& York, 2003, p. 2). Working in conjunction with the other fundamental organizational capacities, adaptive capacity requires nonprofits to act as learning organizations and to use evaluation as a tool to enhance organizational learning and performance (Letts, Ryan \& Grossman 1999; Sussman, 2003).

In spite of its importance, research findings conclude that adaptive capacity tends to be the weakest organizational capacity among nonprofits (Connolly \& York, 2003). Two essential aspects of adaptive capacity, the creation of a learning organization and the implementation of evaluative practices, both present significant organizational challenges. Moreover, when nonprofit organizations do receive help in building particular aspects of organizational capacity, they do not necessarily focus on strengthening their adaptive capacity (Connolly \& York, 2003). These particular challenges to effective adaptive capacity building among nonprofits are outlined in Figure 2.

Figure 2: Barriers to Influencing Social Change

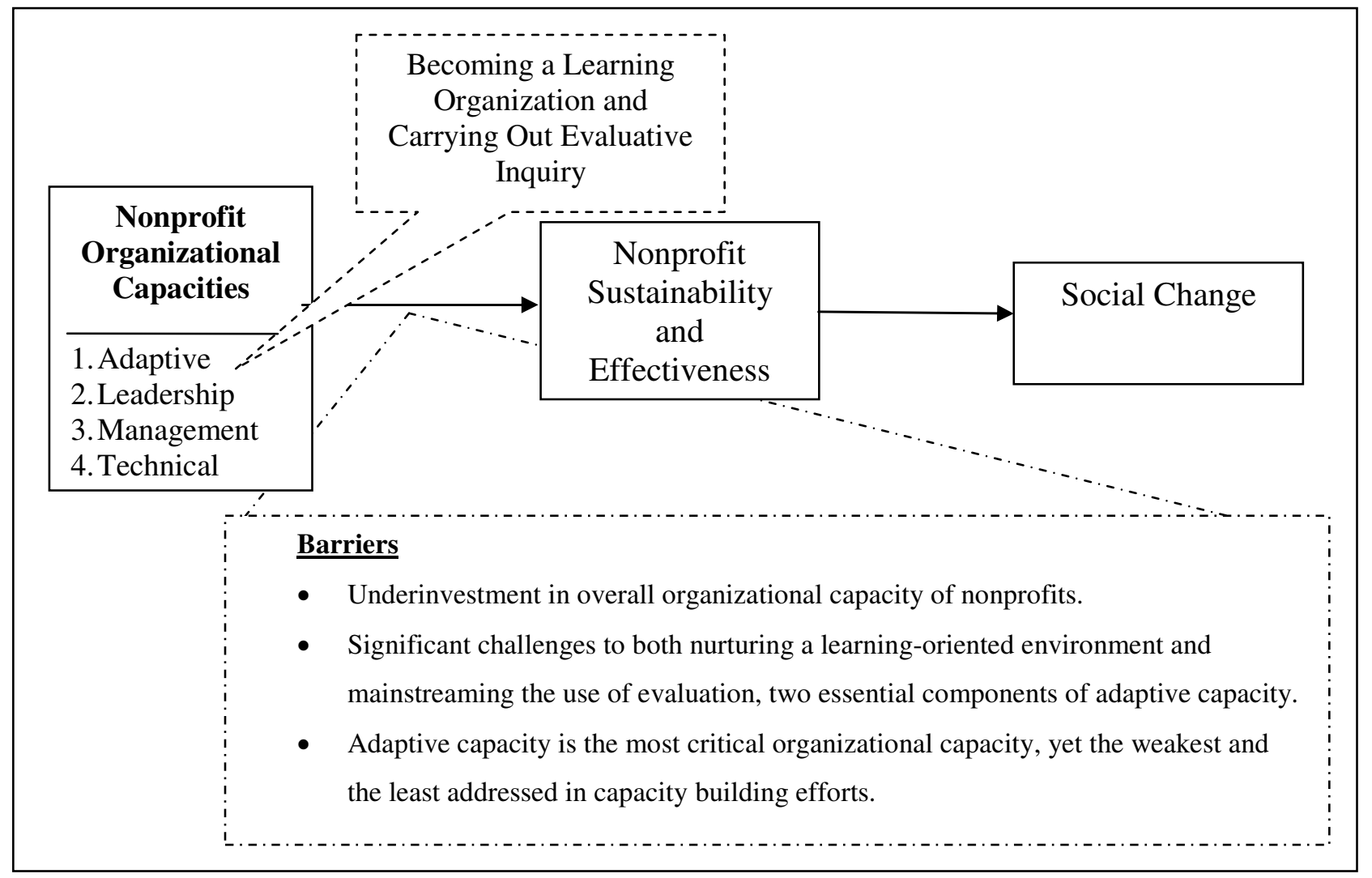


If adaptive capacity is so essential to nonprofits, why does it not receive greater emphasis among those seeking to enhance overall organizational performance? Highlighting a mismatch that occurs between the types of capacity building efforts provided and the actual needs of the nonprofits, research findings cite a lack of organizational readiness as one of the major reasons for this resulting mismatch (Connolly \& York, 2003, p. 39). The importance of gauging the level of organizational readiness prior to engagement in all types of capacity building efforts is highlighted in the research (Blumenthal, 2003; Connolly \& York, 2003; Fine, Kopf \& Thayer, 2001). Yet, at the same time, there is little understanding in the field regarding what makes a nonprofit 'ready' to engage in the process of capacity building (Connolly \& York, 2003). At a minimum, nonprofits need to be open to the idea of change and be willing to invest the necessary time in the long-term process of building their capacity. With particular regard to building adaptive capacity, the requirements placed on the nonprofit for successful engagement may be more demanding. Ensuring adaptive capacity appears to be much more complicated than upgrading a nonprofit's computer system or improving the coordination of its fundraising efforts. The organizational culture, and whether or not it is conducive to learning and the use of evaluation, should continually be assessed throughout the process of improving adaptive capacity. In determining readiness, it is imperative to explore the organization's current learning capabilities and gain more insight into how the organization functions in terms of its

'organizational culture.' What is learned from the process is continually fed back into the cycle of learning; the nonprofit has the ability to continually evaluate itself, guide change, and adapt to its changing environment.

\subsection{OVERVIEW OF THE STUDY}

Drawing from various fields of inquiry, including organizational learning, evaluative inquiry and nonprofit capacity development, this descriptive cross sectional study sought to develop a conceptual framework for understanding organizational readiness to improve adaptive capacity. Having gained significant momentum only in recent years, capacity building efforts among nonprofits have yet to become the focus of extensive research. As discussed above, there is little 
understanding about what makes an organization 'ready' to engage in the process of capacity building, and there is also a lack of research in terms of understanding adaptive capacity (Connolly \& York, 2003). The research study was designed to make a contribution by exploring the perspectives of the nonprofit regarding their adaptive capacity.

The study therefore addressed the following research questions:

1. How do the nonprofit staff and board members perceive the adaptive capacity of their respective organizations?

2. What are important factors that can be identified as either facilitators or barriers to the ability of these nonprofits to promote their adaptive capacity?

The following chapter, Chapter Two, represents an effort to provide an overall theoretical framework of organizational readiness for adaptive capacity building. First, the literatures about learning organizations and participatory evaluation are reviewed, followed by a review of selected organizational assessment tools. The discussion then turns to the unique context of nonprofit organizations, and factors such as the organizational culture of nonprofits, their funding environment, and the role of capacity building (particularly adaptive capacity building). In the final section of Chapter Two, these various fields of inquiry are synthesized and the implications for the study are discussed. In Chapter Three, the methodology that was used to examine the research questions outlined above is described. The chapter begins by exploring the context of the study and the guiding research questions and their rationale. This is followed by a description of the site selection, the data collection and data analysis techniques, the pilot test, the limitations and strengths of the study, and the presentation of the data. In Chapter Four, the results of the research findings, as according to the theoretical framework of adaptive capacity, are presented. Lastly, in Chapter Five, the purpose and scope of the study are reviewed, and the summary and conclusions, directions for future research, and implications of the study are discussed. 


\subsection{LITERATURE REVIEW}

A nonprofit organization with strong adaptive capacity is an organization that first, understands the ongoing challenge towards becoming a learning organization and, second, appreciates the importance of evaluative thinking. The readiness of an organization to engage fully in these processes is critical, and its success can hinge on the willingness and commitment of the organization to facilitate change. While adaptive capacity is considered to be a fundamental component for enhancing organizational effectiveness, it is a particular aspect of organizational capacity for nonprofits that requires more research (Connolly \& York, 2003). As a relatively new concept, adaptive capacity is generally used in this study in order to refer to a nonprofit's ability to act as a learning organization and to apply evaluative inquiry. Whereas there are very few studies on the topic of adaptive capacity for nonprofits, there is extensive literature on learning organizations and evaluation use; the literature on these topics therefore serve as the main source of developing an overall conceptual framework for organizational readiness.

This chapter helps to frame this study by first exploring in depth the fundamental concepts associated with and the characteristics of a learning organization. A discussion on the principles of participatory evaluation and the various approaches to evaluation as a process to support learning follows. Existing instruments (Davidson, 2001; Watkins \& Marsick, 1999; Preskill \& Torres, 2000b) for assessing an organization's learning culture are then reviewed. The next step is to consider how these general concepts can be applied to the unique context of the nonprofit organization. Certain factors, including the organizational culture of nonprofits, the funding environment, and the role of capacity building (particularly adaptive capacity building), are then examined. The final section of the chapter synthesizes these various fields of inquiry and discusses the implications for this study. 


\subsection{LEARNING ORGANIZATIONS}

It is instructive to begin by creating a conceptual framework of learning organizations, drawing from literature of organizational learning (e.g.; Argyris \& Schon, 1996; Senge, 1990; Garvin, 2000), informal learning (Marsick, Volpe \& Watkins, 1999; Volpe \& Marsick, 1999; Raelin, 1999), social capital (Cohen \& Prusak, 2001; Putnam, 1993), communities of practice (Wenger, McDermott \& Snyder, 2002; 2001) and knowledge management (Davenport \& Prusak, 1998); Seely Brown \& Duguid, 2001). A critical process that need to take place for learning to occur, referred to as 'transformational learning', is first explored. The underlying assumptions identified in the literature as essential to learning organizations and the kind of environment that facilitates organizational learning are then considered.

The majority of organizations certainly would like to consider themselves as learning organizations. Indeed, as Raelin tells us, "any organization that has survived within our current turbulent global environment is already a learning organization in one form or another" (p. 28). While Preskill and Torres (1999) affirm that in many respects all organizations learn, they emphasize that "how they learn, why they learn, and how they apply their learning varies significantly" (p. 44). Learning may be taking place, but the question is: what kind of learning? Is the learning process deliberate? Efficient? Systematic? Does it challenge the organization to collectively assess its underlying assumptions? Does the knowledge lead to a change in how the work gets done? As Coutu (2002) surmises, "most people end up doing the same old things in superficially tweaked ways - practices that fall far short of the transformational learning that most organizational experts agree is the key to competing in the twenty-first century" (p. 100).

How does this process of 'transformational learning', which is described as difficult, time consuming and highly anxiety-provoking, actually take place (Schein, 1992)? This process of transformational learning is discussed in the following section. In doing so, it is important to consider that transformational learning is only the first basic step in the journey toward becoming a learning organization. 


\subsubsection{Exploring Transformational Learning}

What is transformational learning? Transformational learning makes us examine and reassess the underlying assumptions guiding our behavior and practice ${ }^{1}$. As Raelin (2000) explains, it is the kind of learning that "brings us to new meaning" and allows us to recognize the paradigmatic assumptions in our thinking (p. 60). It is worthwhile to briefly consider the role of anthropologists; they 'step out' of their own context and are thereby able to identify a panoply of norms, values, beliefs, worldviews and ideologies (Anderson, 1990). As the anthropologists Monaghan and Just (2000) write, "the outsider is far more likely to notice the tacit understandings that local people take for granted as 'common sense' or 'natural' categories of thought" (p. 30). To achieve transformational learning, we also need to 'step out' of our context. In order to "make the familiar strange" (Schram, 2003, p. 58), we need to understand that the meaning we attach to knowledge depends on our values, beliefs and world view.

How open are we to new ideas? How often do we evaluate new perspectives or dissenting views? Our first challenge is to be able to acknowledge our worldview, commonly termed in the literature as "mental models" (Senge, 1990) or "theories-in-use" (Argyris \& Schon, 1996). This is a very multifaceted and complex process. As Senge informs us, "We do not 'have' mental models. We 'are' our mental models" (Senge, p. xv). Describing our mental models as our "deeply ingrained assumptions, generalizations, or even pictures or images that influence how we understand the world and how we take action", Senge explains how frequently we are unaware that these models exist and that they shape us or our perceptions or behavior (p. 8). In the words of William James, "A great many people think they are thinking when they are merely rearranging their prejudices" (quoted in Garvin, p. 216). We tend to overestimate how 'open' we really are (Garvin, 2000). And while our assumptions may not necessarily be right or wrong, the problem is that they generally remain tacit, and therefore unexamined (Senge, 1990). Transformative learning requires us to confront our beliefs and assumptions in our thinking and reflect on how they influence our actions.

\footnotetext{
1 Citing Mezirow (1981), Raelin (2000), and Ziegler (1999) this type of learning is also described as "transformative". The general term "transformational" is used to refer to the kind of learning that challenges paradigmatic assumptions.
} 
According to constructivist learning theory, individuals learn through a process of meaning making (Mezirow, 1991), interpreting and understanding their experiences in ways that affect their worldview. Constructivist learning, which suggests that "individuals continually create and re-create meaning as a result of their relationship with others in the social environment" (Preskill \& Torres, 1999, p. 19), often leads individuals to be transformed by their experiences (Preskill \& Torres, 2000a, p. 28). Both constructivist learning and transformational learning can be described as a process of 'making meaning', where learners regularly and proactively assess their experiences and their interpretations of 'knowledge'. For this kind of learning to occur, one has to make assumptions about reality explicit and then be willing to challenge one's beliefs. This challenge is "at the heart of change" (Ziegler, 1999, p. 57).

A brief review of Argyris and Schon's (1996) discussion of single loop and double loop learning can provide further insight into the process of confronting one's deeply held assumptions and beliefs. With single loop learning, learning takes place when there is a gap between the intentions and the outcome. An individual will reflect on this mismatch between actions and outcome, and consider a change in tactics. The result in single loop learning is a different strategy or action. What does not take place is a deeper level of reflection, which would lead the individual to reassess the values, beliefs or assumptions that are associated with the action itself (Argyris \& Schon, 1996). Preskill and Torres (1999)'s discussion of single loop and double loop learning at the organizational level describes this type of learning as one that is "used to maintain the status quo and is often employed in crisis situations" (p. 44). Arguing that it generally leads to "short term solutions to organizational problems", the authors cite change efforts such as the Total Quality Management or reengineering as examples of popular fads that leave intact the underlying assumptions that govern organizational behavior (p. 44) ${ }^{2}$. As Argyris and Schon (1996) tell us, only when there is an evidence of a change in an organization's 'theoryin-use', which ultimately shapes its identity, does organizational learning occur.

\footnotetext{
${ }^{2}$ Preskill and Torres (1999) believe that these change efforts, cited as examples of single loop learning, have failed because the organizations "have not factored in organization's culture, the ways in which employees change, and how people learn" (p. 12). The values and norms underlying the operations and behavior of the organization remain unchanged.
} 
Double loop learning, in contrast, includes "follow ups" (Argyris, 2001, p. 91). The individual questions not only the strategy but the reasoning and motives that underlie the action ${ }^{3}$. Only through double loop learning can an organization explore the values and norms that are determining its 'theories-in-use' (Argyris \& Schon, 1996). Members of the organization need to be able to explore the reasons for things, not just the "how" but the "why": "understanding the 'whys' is at the heart of learning" p. 56 (Ziegler, 1999).

In continuing to explore the meaning of transformational learning, it is important to keep in mind that mental models or theories-in-use can be individual or organizational (Ziegler, 1999; Argyris \& Schon, 1996). On an individual level, surfacing and questioning one's mental models or theories-in-use is not a process in which people necessarily take pleasure. As Schein (quoted in Coutu, 2002) reminds us “it's just not a joyful process to give up your values and beliefs" (p. 103); we do not naturally seek to change the attitudes that we hold. Similarly, Senge tells us that it can be both disorienting and frightening to challenge long held beliefs and assumptions. This type of learning "temporarily destabilizes our cognitive and interpersonal world", resulting in high levels of anxiety and defensiveness (Schein, 1992, p. 22). The process of transformational learning can not only frustrate individuals but can affect their self -esteem and, in some cases, even their sense of identity (Schein, quoted in Coutu, 2002). So the question can be asked, is this difficult and complicated process actually necessary? Can it be avoided? In a true learning organization, it is inevitable; organizational learning can only take place when organizational members themselves learn (Senge, 1990, Preskill \& Torres, 1999; Garvin, 2000).

In discussing the question of how it is that organizations actually learn, Argyris and Schon (1996) point to the existence of an "organizational learning system" (Schon, 1983, Argyris \& Schon, 1996). The members of an organization operate within the norms and culture of this 'learning system', which is described as 'the behavioral world 'draped over' its structure, information network and systems of incentives" (Argyris \& Schon, 1996, p. xxiii). A 'learning system' can either foster or inhibit learning at an individual and organizational level. Organizations themselves have the ability to learn when someone, on behalf of the organization, carries out an inquiry addressing the challenges facing the organization (Argyris \& Schon, 1996,

\footnotetext{
3 An example of double loop learning provided by Preskill and Torres (1999) is a nonprofit organization that is experiencing high turnover among volunteer staff. Instead of simply deciding to fix their volunteer training methods, they question if the turnover is a symptom of a larger problem and examine issues such as administrative support, coaching and feedback and workload. This issue is explored further in the section on systems thinking.
} 
Volpe \& Marsick, 1999). After the inquiry, the individual can act on these new insights and share this new knowledge with other members of the organization, thereby creating a "collective learning process" (Volpe \& Marsick, 1999, p. vi). A "collective learning process" that leads to transformational learning will create a willingness on behalf of the organization to be open to innovation (Raelin, 2000; Zeigler, 1999); only through this kind of learning can cultural change take place within in an organization (Schein, quoted in Coutu, 2002). In the following section, the values and norms that characterize a 'learning system' are discussed.

\subsubsection{The "Learning System" of a Learning Organization}

It is possible to extract from the literature a variety of dimensions that are considered as essential for creating a "learning system" that facilitates learning within an organization. The boundaries, a necessity in the creation of a list, are, in a sense, artificial. The categorization is primarily for the sake of discussion, as each of the list items is interrelated, overlapping, and serves to strengthen one other. ${ }^{4}$ As Senge (1990) reminds us in regard to the five disciplines he outlines as fundamental for learning organizations (shared vision, mental models, personal mastery, team learning and systems thinking), "all the disciplines matter" (p. xviii). The following list draws from Senge's five disciplines and other literature on learning organizations, as well as from literature in the field of organizational development, informal learning, knowledge management and communities of practice.

These six essential dimensions of a 'learning system' are each addressed in further detail:

1) Shared Vision/ Shared Meaning

2) Openness and Inquisitiveness

3) Systems Thinking

4) Community of Learners

5) Informal Learning

6) Social Capital

\footnotetext{
${ }^{4}$ For example, a culture that values openness and the accessibility of knowledge will enhance opportunities for informal learning; a community of practice can only thrive in an environment characterized by high social capital; and so on.
} 


\subsubsection{Shared Vision/ Shared Meaning}

Organizations embarking on the journey towards transformational learning encounter an extensive, difficult and anxiety-provoking process (see Section 2.1.1). It takes a great deal for organizations to successfully create an environment that encourages the surfacing of "threatening or embarrassing information that can motivate learning and produce real change" (Argyris, 2001, p. 80). Taking on the conventional wisdom of the organization is inevitably a politically charged process that can expose vulnerabilities at the individual or organizational level. The only predictable part of the process is that it will be not be easy, and "typically, the immediate downside risks and costs of change are more evident than the potential rewards" (Bickel, Millet, $\&$ Nelson, 2002, p. 5). For example, individuals or groups in the organization who do undergo a learning process may not be willing to share their new understandings and potentially create undesired conflict if there is little motivation to do so. Obviously it can be uncomfortable to be disruptive to an organization by questioning accepted norms or challenging the status quo (Garvin, 2000; Raelin, 1999). For a variety of reasons, the forces that seek to maintain the status quo can be overwhelming unless a compelling vision provides a direction (Senge, 1990). This 'shared vision' will foster the risk taking and experimentation necessary to change the status quo of the organization (Senge, 1990). A learning organization needs a shared vision that can encourage the process of transformational learning and act as a "rudder" that keeps everyone on course during an inevitably challenging and frustrating process (Senge, p. 209).

The correct response to a changing environment, according to Collins and Porras (1997), is not to ask, "How should we change" (p. 8)? Instead the question is "What do we stand for and why do we exist" (p. 8)? Shared vision can help provide stability in the midst of organizational adaptation and change. In considering the role of a shared vision, it is important to remember that as, Davenport and Prusak (1998) emphasize, an organization's "agility is necessarily limited" (p. 64). Organizations should undergo transformational learning and continually adapt to change; however they are not "chameleons" (Davenport \& Prusak, p. 64). Change cannot be only for the sake of change. The role of a shared vision can ensure that an organization understands first what is at its core, thereby enabling it to direct the learning process and adeptly respond to changes in its environment. 
Senge writes that "shared vision is vital for the learning organization because it provides the focus and energy for learning" (p. 206). The shared vision of a learning organization naturally should include a vision for the learning process. Garvin (2000) discusses a "learning strategy" that can ensure that learning is purposeful and aligned with the shared vision of the organization. The process needs to be intentional, with the recognition that it will be ongoing and constant. Learning-oriented cultures measure learning processes (Garvin, 2000), and learning is discussed explicitly (Garvin, 2000; Raelin, 1999). According to Garvin, any organization aspiring to become a learning organization should design a strategy with the following three questions in mind:

\section{What are our most pressing business challenges and greatest business opportunities?}

2. What do we need to learn to meet the challenges and take advantage of the opportunities?

3. How should the necessary knowledge and skills be acquired? (p. 15)

As an organization begins to ask these questions and undergoes a process of organizational learning, "shared meaning" emerges as new insights and new understandings come to light. In her case study of organizational change, Ziegler (1999) explores the importance of creating "shared meaning" among members of an organization in the midst of upheaval and change. Shared meaning evolves as "people began to develop new, collective understandings of the organizational world they were shaping" (p. 56). As members of the organization began to reframe their original thoughts or assumptions, gradually the organization began to "operate from a common, collective reference point" (p. 56).

How does an organization construct a "collective reference point"? How does it ensure that each member of the organization can easily answer the question, "What do we want to create?" (Senge, 1990, p. 206). Emphasis needs to be placed on communication, a critical element to an organization's wellbeing (Schein, 1992; Torres et al., 1996) that includes dialogue and reflection to help regularly facilitate learning. In recent years, storytelling, for example, has become increasingly recognized as a powerful mechanism to bring about collective understanding and knowledge exchange (Seely Brown \& Duguid, 2001) ${ }^{5}$. Cohen and Prusak (2001) discuss the

\footnotetext{
${ }^{5}$ Qualitative research on organizations, which is enjoyed a growth in reputability, is considered especially useful for identifying causal links between variable such as learning, knowledge and performance (Wenger, McDermott \& Snyder, 2002, p. 253). For the use of narrative in the field of evaluation, see Costantino \& Green (2003)
} 
importance of "social talk", which includes storytelling as a means for conveying norms and values, strengthening the connection between members of an organization and allowing them to reach shared understandings. More specifically, stories about the organization "help tie together individual identity and organizational identity", and "create a sense of membership and engagement" (p. 116). A shared vision that can engage members of an organization and generate a sense of commitment is essential in the change process, providing an 'anchor' as the change is navigated with a sense of innovation and inquiry. It is this next dimension of learning organizations, openness and inquisitiveness, that is now discussed in the following section.

\subsubsection{Openness and Inquisitiveness ${ }^{6}$}

A spirit of inquiry, the second dimension of a learning oriented culture, helps to reduce "learning anxiety"; "Learning anxiety", as Schein (quoted in Coutu, 2002) explains, originates from "being afraid to try something new for fear that it will be too difficult, that we will look stupid in the attempt, or that we will have to part from old habits that have worked for us in the past" (p. 104). The anxiety inherent in the learning process can be lessened when a "psychologically safe environment" is created that provides a safe space for learning (Schein, quoted in Coutu, 2002, p. 105). An organization that values openness and inquisitiveness can help to mitigate the angst created by the learning process.

Achieving genuine openness, which is necessary if collective inquiry is to take place (Wenger, McDermott \& Snyder, 2002; Senge, 1990), is not easy. This process can be even more difficult in the absence of signs of stress or an impending crisis; "success is often the enemy of innovation" (Davenport \& Prusak, 1998, p. 63). It can be even more challenging to confront cherished beliefs when they have worked so well in the past. In order to create a learningoriented culture, an organization's leadership should nurture an environment of openness in which individuals feel comfortable asking questions and discussing their inadequacies and mistakes (Garvin, 2000; Raelin, 2000). Leaders set the tone by being learners themselves, demonstrating a willingness to recognize their own vulnerabilities (Schein, quoted in Coutu, 2002). A safe environment is essential for organizational members to feel that the reassessment of the organization's theories-in-use is valued and will be rewarded. Curiosity and

6 Garvin (2000) tell us explicitly that "organizational learning demands inquisitiveness and openness" (p. 19). 
experimentation have to be encouraged (Garvin, 2000; Volpe \& Marsick, 1999), and individuals should have the opportunity to meet new people, experience new challenges and be introduced to new ideas (Volpe \& Marsick, 1999).

Garvin (2000), in the context of a discussion of the three stages of organizational learning, (1. acquiring, 2. interpreting and 3. applying information) identifies "learning disabilities" that can inhibit the learning process. In the first stage of learning, the process of acquiring information, there is a challenge in separating the "signals" from the "noise". (Garvin, p.21) Garvin explains the need "to distinguish relevant from irrelevant information, while remaining open to unexpected, and occasionally unwelcome, surprises" (p. 21). One of the learning disabilities during this process of knowledge acquisition is the propensity to search for information that confirms already held viewpoints. Information is collected within an existing framework, resulting in oversights or errors in the data collection process; "much of what people would label as information only reaffirms old news" (Kiesler \& Sproull, 1982, quoted in Garvin, p. 23).

A second "learning disability", occurring in the second stage of the learning processinterpreting information- is the tendency to filter the information through the lens of one's "mental models" or "theories-in-use". While our mental models help us to give knowledge meaning, these interpretative frameworks are "invariably sketchy and incomplete" and can "become obsolete" (Garvin, p. 25). It is much easier to avoid the anxiety inherent in the process of confronting our cherished beliefs and assumptions (see Section 2.1.1). In our effort to have both "stability and meaning", it is more convenient to "to distort new data by denial, projection, rationalization or various other defense mechanisms than to change the basic assumption" (Schein, 1992, p. 27). Ongoing learning requires us to be vigilant about recognizing our "mental models" and their impact on our thoughts and behavior.

To guard against certain learning disabilities, an organization has to actively pursue new sources of information and knowledge, proactively scan its internal and external environment, and ensure that there is an openness to both diverse opinions and discordant information (Garvin, 2000). The importance of posing questions and cultivating a sense of inquiry among organizational members cannot be underestimated. As the "motive and force that gives shape to inquiry" (Garvin, p. 207) questions can spark reflection, helping individuals to pursue new ways of thinking and reassess their interpretative frameworks. Schein (1992) tells us that a "learning culture must contain the shared assumption that solutions to problems derive from a pragmatic 
search for truth and that truth can be found anywhere, depending on the nature of the problem" (p. 366). Knowledge can be found in all places and in all levels of the organization, and learning is considered the responsibility of everyone in the organization. Recognizing that there is not only one source or means for discovering the truth, learning organizations welcome a diversity of thought. They seek out multiple perspectives and do not shy away from potential conflict that may arise as a result. When there is in an environment that is conducive to learning, risk taking and innovation, 'dissension' itself can lead to more productive learning (Wenger, McDermott \& Snyder, 2002).

The third and final stage of the learning process, the application of information, is accompanied by its own challenges and "learning disabilities". According to Garvin (2000), as a result of inertia and avoidance of risk taking, "the primary problem (at this stage) is passivity- an inability or unwillingness to act on new interpretations" (p. 33). To help overcome this disability, an openness to experiment ${ }^{7}$ and to take risks (and to potentially make mistakes) is essential. In learning-oriented cultures, there is importance attached to recognizing, reflecting upon and learning from errors, mistakes and occasional failures that are used as learning opportunities rather than find the 'guilty' or the 'scapegoats' (Garvin, 2000). Error is tolerated as a necessary part of experimentation and innovation, and the lessons learned from the mistakes are valued and shared among the organization's members (Garvin, 2000). Senge (1990) tells us that the practice of forgiveness results in genuine support for risk taking. In learning organization, there is forgiveness; for "making the mistake is punishment enough." (Rollwagen, quoted in Senge, p. 301).

How does an organization convey this openness, encouraging a sense of inquiry and of experimentation among its members? To start, organizational members have to feel confident that the leadership will not, as Garvin (2000) puts it, "shoot the messenger" for bringing bad news (p. 13). They need to feel listened to and have a comfort level to raise problematic issues and to reflect on mistakes.

\footnotetext{
${ }^{7}$ Garvin (2000) distinguishes between processes that have an orientation towards the past, present and future (p. xii). Experimentation pushes an organization to tests potential theories or models for future application. Whereas experimentation is focused on the future, intelligence gathering is aimed at understanding the present - collecting the information that is available at the current time. Experiential learning, on the other hand, explores what has occurred and how the organization can learn from its past.
} 
To create this type of environment Garvin identifies three essential conditions:

1) A culture that does not demand infallibility and perfection; ${ }^{8}$

2)Freedom to fail without punishment or penalty; and

3) Systems or incentives that encourage the identification, analysis and review of errors (p. 40). ${ }^{9}$

In addressing the challenge of learning from our mistakes, Senge (1990) discusses the dilemma that "we learn best from experience but we never directly experience the consequences of many of our most important decisions" (p. 23). The impact of our action can potentially affect something or somewhere else in the 'system' and, to further complicate matter, the impact of the action may not be felt right away. Systems thinking, "a discipline for seeing wholes", acknowledges that we operate in "complex systems" where the consequences of our actions are removed from us in time and space, and the feedback is not rapid and unambiguous (Senge, p. 313). The discipline of systems thinking can help learning organizations to understand how particular decisions and actions affect the 'whole' and how systematic change takes place.

\subsubsection{Systems Thinking}

Systems thinking, which Senge (1990) refers to as "the cornerstone of how learning organizations think about their world" (p. 69), gives us the ability to operate with "a framework for seeing interrelationships rather than things, for seeing patterns of change rather than static "snapshots" (Senge, 1990, p. 68). To gain a very cursory understanding of systems thinking, it is possible to consider key attributes of 'complex systems', while keeping in mind that organizations are defined in these terms as well (Eoyang \& Berkas, 1998; Senge, 1990).

Complex systems are characterized by interdependency; each component influences, and is influenced by, the behavior of other components in the system (Sterman, 2001; Eoyang \& Berkas, 1998). Systems thinking recognizes the interaction and the relationship among the various parts of the system (often referred to a coupling). Rather than operating as a collection of

\footnotetext{
${ }^{8}$ In the context of a discussion of corporate culture at IBM, Garvin explains how its founder, Watson Sr. understood that "demanding infallibility is a prescription for paralysis" (p. 41).

${ }^{9}$ As Garvin (2000) stresses, even when there is a supportive learning environment it is often difficult to isolate variables or understand the nature of cause and effect (p. 100). The meaning of "systems thinking" will be further explored in the upcoming section.
} 
separate, independent parts, the components are interdependent and can lose their capacities if separated from its system. Several old adages convey this perspective such as "if you cut a cow in half, you don't get two cows" or "the whole is greater than the sum of its parts" (cited in Sweeney, 2001, p. 23).

The interactions in a complex system create qualities or properties as a whole, which is very different from adding each component separately. "Emergent property" is the term used to describe "a property or behavior that arises only out of the interactions within a specific set of parts" (Sweeney, 2001, p. 24). The behavior or properties of individual components of the system work together to create the behavior of the whole system. Senge discusses the idea of team synergy, using the Boston Celtics as an example of "alignment", which he defines as occurring "when a group of people function as a whole" (p. 234). Figure 3 illustrates the difference between a group and a team (or system). The quality of the interaction makes the team great, and helps to explain, as Sweeney tell us, "why the all-star team is not always the best team in the league" (p. 24). The sum of the parts is not as relevant as the interaction of the parts and how the system "plays" together. Babe Ruth can summarize this for us: "You may have the greatest bunch of individual stars in the world, but if they don't play together, the club won't be worth a dime" (quoted in Cohen \& Prusak, 2001). ${ }^{10}$

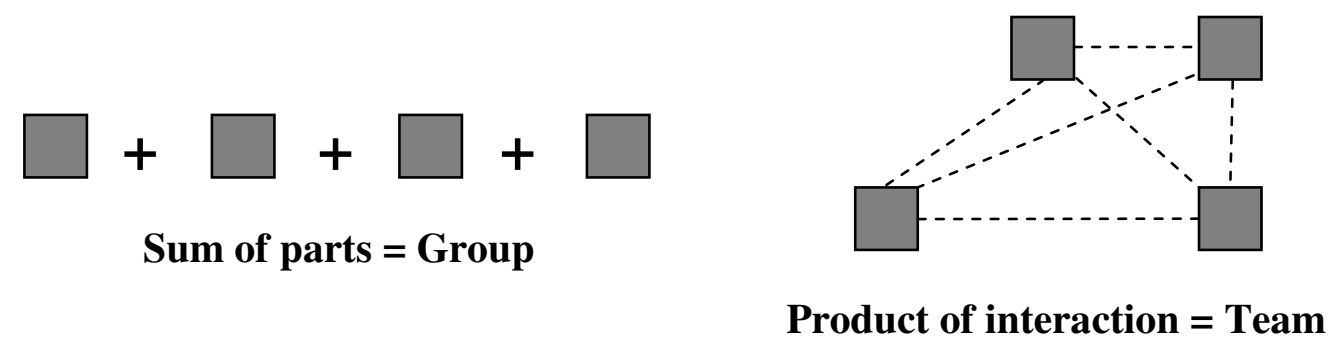

Figure 3: "The Whole is Greater than the Sum of Its Parts" (Sweeney, 2001)

Complex system are also dynamic and nonlinear (Sterman, 2001). When cause and effect are linear, one event directly leads to the next event, like a set of falling dominoes (Sweeney, 2001). In complex systems, on the other hand, the cause and effect is not linear; rather cause and effect

\footnotetext{
${ }^{10}$ The quote is used by Cohen \& Prusak (2001) to illustrate the importance of social capital, a topic that will be discussed more in depth.
} 
is depicted as circular with feedback loops. Feedback loops are described as the tendency of a system to use its own input to make adjustments in its inputs and /or processes (Sterman, 2001; Eoyang \& Berkas, 1998). As "self-organizing and adaptive" (Sterman, 2001), a system has circular causality where "one event causes another event and that second event comes back around to influence the first cause" (Sweeney, 2001). Figure 4 illustrates that, in contrast to cause and effect that is linear (A causes B causes C), systems are characterized by circular cause and effect: A causes B, B causes C and C causes A.

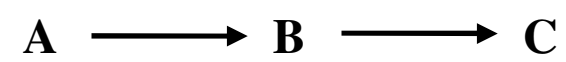

Linear Causality

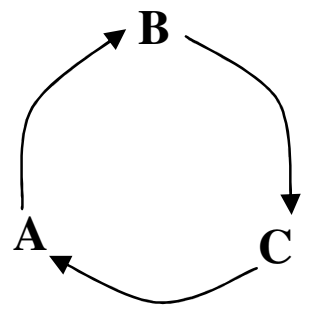

Circular Causality

Figure 4: Illustrations of Cause and Effect (Sweeney, 2001)

"Systems thinking tells us to stop and look below the surface, to see how the structure (the relationship between the parts of the system) drives the patterns of change we see" (Sweeney, 2001, p. 31). As previously discussed in the context of single loop learning versus double loop learning, it is easier to react to events and respond with immediate action (the mentality of "putting out fires"), than to take the time to reflect on how the event fits into a larger pattern. Yet, while it is necessary to address the underlying causes of events and anticipate unintended consequences, our mental models are, according to Sterman (2001), "structurally simplistic." Our mental models have "short term horizons" and suffer from narrow boundaries or "silo" thinking (Sterman, 2001). This tendency to focus on events and look for causes that are closely related in time and space consequently leads to problem solving that is linear. We overlook the fact that "problems don't stand still" (Sweeney, 2001, p. 20) and neither do systems. Sterman (2001) explains how linear problem solving does not work in complex systems, where the decisions we make are affected by the environment that also includes, for example, 'side effects', 
goals of others, actions of others and time delays. ${ }^{11}$ The 'side effects', which are unanticipated, can end up working against the desired outcome, or simply shift the problem to a different part of the system or into the future (Senge, 1990). ${ }^{12}$ What is forgotten is that the system is dynamic, unpredictable and governed by its own feedback.

"Because of the number of agents, their interdependence, and their openness to external influences, a complex (adaptive) system changes constantly and continuously" (Eoyang \& Berkas, 1998, p. 4). Systems thinking requires an analysis of multiple perspectives and on multiple levels in order to gain an understanding of how the relationships between the parts of the system constantly interact, 'self-organize' and create unpredictable change. Since it is impossible to foresee the future state of the system, alternative possibilities for outcomes need to be considered. Furthermore, because "systems don't demonstrate a full 'cycle' of their behavior over short periods" (Sweeney, 2001, p. 34), one has to take a long term view, and understand the 'delays' in the system. Sterman (2001) outlines several suggestions to consider during the decision making process as a means to ensure that one is adopting systems thinking; such as expanding one's mental models, focusing on feedback, understanding dialogue and selfreflection, practicing empathy, continually moving between assessment and action, and seeking multiple points of view. Communities of practice and other networks are considered ideal places for nurturing these types of processes.

\subsubsection{A Community of Learners}

Raelin (1999) tells us that while learning takes place on an individual level, learning in the workplace "requires an extension of learning out to the collective level defined as one's coworkers" (p. 54). Senge (1990) goes even further, arguing that individual learning is practically "irrelevant" (p. 236) to organizational learning. He asserts that team learning is critical because "teams, not individuals, are the fundamental learning unit in modern organization" (p. 10); when teams learn, "they become the microcosm for learning throughout the organization." (p. 236). The variety of names given to groups that facilitate teamwork include communities of interests, communities of practice or knowledge networks (Wenger,

\footnotetext{
${ }^{11}$ Sterman (2001) argues that in reality, there are no "side effects," there are only "unintended effects."

${ }^{12}$ For example, the heavy patrolling of one section of a neighborhood to reduce drug crime simply results in the shifting of the drug crime to a different section that is under less surveillance.
} 
McDermott \& Snyder, 2002; Raelin, 1999; Davenport \& Prusak, 1998). Increasingly, organizations encourage the establishment of these types of social groups as a means for "moving" knowledge throughout the organization (Davenport \& Prusak, 1998). ${ }^{13}$ These communities demonstrate tangible results in knowledge production and transfer, and also lead to intangible outcomes such as the creation of relationships, the strengthening of professional confidence and the nurturing of a sense of belonging and trust among its members (Wenger, McDermott \& Snyder, 2002). Helping to generate a spirit of inquiry, a community of learners can provide its members with an important platform by which to explore assumptions (Wenger, McDermott \& Snyder, 2002). A network of relationships that is characterized by trust and a mutual sense of identity will help to mitigate the effects of "learning anxiety."

Wenger, McDermott and Snyder (2002) discuss how "knowledge involves the head, the heart and the hand; inquiry, interactions and craft. Like a community, it involves identity, relationships, and competence; meaningfulness, belonging and action.”(p. 45). They suggest that a community of practice ${ }^{14}$, an example of an informal network, "matches" the complexity that is associated with knowledge (p. 45). Informal learning and teamwork, which require interface and conversation, tap into the collective nature of knowledge. Knowledge is not only dynamic but is "interactive", and is based on the creation of "shared meaning" (Raelin, p. 11). ${ }^{15}$ In considering the social construction of knowledge, it is important to remember the communal role in influencing our "mental models" or "theories-in-use". Kuhn (1962) highlights the critical

\footnotetext{
${ }^{13}$ Cohen and Prusak (2001) identify the overlap between networks and communities: both can be described as "groups of people brought together by common interests, experiences, goals or tasks; both imply regular communication and bonds characterized by some degree of trust and altruism" (p. 56). While acknowledging differences between them (see Cohen \& Prusak, 2001), the terms are used interchangeably.

14 There is a growing interest in organizational infrastructures such as communities of practice to achieve organizational learning. While the focus is specifically on communities of practice existing in the workplace, these communities are generally defined as "groups of people who share a concern, a set of problems or a passion about a topic, and who deepen their knowledge and expertise in this area by interacting on an ongoing basis." (Wenger, McDermott \& Snyder, 2002, p. 4)

${ }^{15}$ It may be worthwhile, at this stage, for us to distinguish between data, information and knowledge. (Davenport \& Prusak (1998) describe data as a "set of discrete, objective facts about events" and information as "data that makes a difference" (pp. 2-3). As the "raw material," data needs to be given meaning: it must be both interpreted and placed into a context in order to become information and actually inform the recipient. Knowledge is much more complex, "knowledge is a fluid mix of framed experience, values, contextual information and expert insight that provides a framework for evaluating and incorporating new experiences and information" (Davenport \& Prusak, p. 5) Knowledge management is not only about the exchange of data but the exchange of knowledge, a process which is impacted by a variety of human and social factors (Davenport \& Prusak, 1998).
} 
significance of a community in shaping the values and beliefs that are accepted as the current 'paradigm'. As Kuhn explains "observation and evidence cannot alone determine a particular body of such belief. An apparently arbitrary element...is always a formative ingredient of the beliefs espoused by a given scientific community at a given time" (Kuhn, p. 4). This recognition of knowledge as collective is essential in organizational learning. As Zeigler (1999) suggests, the sharing of knowledge leads to the creation of collective meaning, a process that ultimately links individual learning with organizational learning.

The process of collective learning at a group level, where shared meaning is created, begins with dialogue. In dialogue, participants first hold judgment as they are exposed to multiple perspectives. Individuals become "observers of their own thinking" (Senge, 1990, p. 242). The next step is to focus on a "commitment to inquire together" that leads to the construction of new meanings and new insights (Raelin, 2000, p. 81). The importance of dialogue is that "individuals gain insights that simply could not be achieved individually" (Senge, p. 241). Ziegler's (1999) study of organizational change concludes that dialogue at the group level allows knowledge to be shared, leading to the creation of "shared meaning from common interpretation of events", and ultimately to organizational change (p. 56). Without group dialogue, Ziegler explains, "knowledge resides mainly in the heads of individuals" without the incentives to teach one's knowledge or to acquire new learning in order to share with others (p. 56).

Collective opportunities for learning can help organizations ensure that both tacit and explicit knowledge is "institutionalized," thereby becoming codified, dispersed and recognized as the "common property" of the organization (Garvin, 2000, p. 15). "Actual work practices are full of tacit improvisations" that are difficult to articulate (Seely Brown \& Duguid, 2001, p. 50). These "tacit improvisations" stand in contrast to explicit knowledge that is expressed and codified. The gap between our "theory-in-use" and our "espoused theory" (Argryis, 1998; Argyris \& Schon, 1996) poses a significant obstacle to organizations seeking to institutionalize tacit knowledge. Individuals are generally unaware of the inconsistency between their espoused theory and theory-in-use, and the fact that their espoused theory is not necessarily an indication of how the individual actually behaves in practice (Argryis, 1998). There is a dissonance between what individuals "think they do and what they really do" (Seely Brown \& Duguid, 2001, p. 50). The 
challenge of learning organizations is how to tap into tacit knowledge, identify theories-in-use, and get "local knowledge into wider circulation" (Seely Brown \& Duguid, 2001, p. 55). ${ }^{16}$

According to Schon (1983), professional knowledge cannot keep up with the demand of professional practice that is characterized by "complexity, uncertainty, instability, uniqueness and value conflict" (p. 18). The process of reflection is therefore essential. Termed by Schon as "reflection-in-action", this process enables practitioners to tap into the tacit knowledge that they automatically utilize as they carry out their work. The process of reflection provides individuals with insight into their "spontaneous, intuitive performance" (p. 49) While "reflection -in-action" is vital, collective reflection-in-action is also necessary. (p. 243). Without it, learning remains private and inaccessible. As Schon explains, "awareness of one's intuitive thinking usually grows out of practice in articulating it to other" (Schon, p. 243). Interaction with others is essential for surfacing both the explicit and tacit knowledge, and collective inquiry creates the opportunity for both knowledge transfer and knowledge generation. Preskill and Torres (2000a) outline a list of factors that serve to ensure the effectiveness of communities of practice (p. 29), as illustrated in Table 1. The following suggestions to enhance communities of practice are relevant to the success of other forms of collective learning as well:

Table 1: Effective Communities of Practice (Preskill \& Torres, 2000a, p. 29)

\begin{tabular}{|l|}
\hline Have the time and space for learning \\
\hline Pay attention to goals and significant organizational events \\
\hline Have accurate and complete information \\
\hline Are able to weigh evidence and assess arguments \\
\hline Are open to alternative perspectives \\
\hline Reflect critically on presuppositions and their consequences \\
\hline Trust the process of working with others \\
\hline $\begin{array}{l}\text { Are able to accept and informed, objectives and rational consensus as a } \\
\text { legitimate test of validity }\end{array}$ \\
\hline Take action on organizational issues (Marsick and Volpe, 1999) \\
\hline
\end{tabular}

\footnotetext{
${ }^{16}$ Whereas data bases are the "most basic" of knowledge management tools for the dissemination of information, they "are also among the most ignored" (Seely Brown \& Duguid, 2001, p. 56). Increasingly, the field of knowledge management has placed emphasis on the importance of human interaction (Davenport \& Prusak, 1998).
} 
It is important to remember, however, that the organizational strengths of teams or informal networks like communities of practice can also be their weaknesses (Wenger, McDermott \& Snyder, 2002). Communities, a forum for collective inquiry, can also act as a barrier to learning when jointly held assumptions are no longer examined; "communities can dig you in deeper or pull you out" (Wenger, McDermott \& Snyder, 2002, p. 157). Wenger, McDermott and Snyder (2002) warn us that the closeness of a community can create bonds that may lead its members to stop seeking external input or initiating explorative efforts. Without continued vigilance, members can continue to reinforce each other beliefs and attitudes, thereby making it difficult to seek new perspectives. Yet, at the same time, communities "create a potential for organized change far beyond the individual capacity to change" (Wenger, McDermott \& Snyder, 2002, p. 157). As discussed earlier, group learning that takes place through dialogue can generate collective understandings and new meanings. A community that embraces the need to change can help its individual members overcome inertia and imbue each other with sense of inquiry (Wenger, McDermott \& Snyder, 2002, p. 157). Networks such as communities of practice represent an example of informal phenomena at the workplace that are increasingly seen as essential for the cultivation of a learning organization.

\subsubsection{Informal Learning}

A "learning system" that facilitates learning will view inquiry as part of everyday work and as integrated into the "doing", mirroring a perspective discussed in theories of informal and workbased learning (Raelin, 2000; Volpe \& Marsick, 1999). Learning organizations attach great significance to the learning process, rejecting the common perception that "learning is of questionable value because it diverts individuals' attention away from the 'real work'" (Garvin, 2000, p. 4). Organizational environments that inhibit learning perceive a disconnect between learning and action, and regard with suspicion those activities that do not "produce immediate, tangible results" (Garvin, p. 4). Indeed, Davenport and Prusak (1998) argue that the next challenge in the knowledge management movement is to ensure that knowledge is not viewed as something separate, but rather as integrated into all the aspects and activities of the organization. Engaging in the learning process becomes part of the work - to "create, gather, store, share and 
apply knowledge" and it becomes the responsibility of everyone in the organization (Davenport \& Prusak (1998, p. xi). ${ }^{17}$

As Raelin (2000) explains, a traditional separation exists between theory and practice, where theory is associated with "the world of thought" and practice represents "the world of action" (p. 50). With a more dynamic view of knowledge and learning, "work-based learning" suggests that learning arises from the work itself; practice and theory merge, and knowledge becomes intertwined with experience (Raelin, 2000). Informal learning becomes central to the experience of work-based learning and knowledge is recognized as something that is drawn from the experiences of everyday life (Raelin, 2000; Wenger, McDermott \& Snyder, 2002). Knowledge is not perceived as an "object" or a "static body of information" but as a "living process" (Wenger, McDermott \& Snyder, 2002, p. 9). Formal training programs or the teaching of theory separate from its context or practice do not necessarily capture the dynamic nature of knowledge and are increasingly viewed as insufficient (Volpe \& Marsick, 1999; Raelin, 2000). Understanding specific tasks or skills is not as significant as being able to adapt in today's organizational environment where jobs can change unpredictably and flexibility is a necessity (Volpe \& Marsick, 1999; Preskill \& Torres, 2000a). There is a growing awareness that the most important skill is not a particular skill set, but rather the ability to adapt and learn in a dynamic context.

Whereas efforts to enhance organization performance have focused traditionally on formal training and classroom instruction, a rising number of organizations are emphasizing the importance of fostering informal learning (Raelin, 2000; Volpe \& Marsick, 1999). Informal networks in organizations are increasingly recognized as critical for knowledge production and knowledge transfer within organizations (see Subsection 2.1.2.4). In fact, Volpe and Marsick (1999) contend that "informal learning has always been the most pervasive type of learning in the workplace" (p. 3). A recent study by the U.S. Department of Labor concluded that over $80 \%$ of learning at the workplace is informal learning (cited in Cohen \& Prusak, 2001, p. 170).

\footnotetext{
${ }^{17}$ The idea of each organizational member as involved in knowledge production and dissemination is referred to by Senge (1990) as "localness"; individuals are given the "freedom to act, to try out their own ideas and be responsible for producing results" (p. 288).
} 
Indeed, Wenger, McDermott and Snyder (2002) argue that "informal phenomena professional passion, relationship and identity - are now the frontiers of management" (p. 217). Increasingly, organizations are recognizing the importance of encouraging their members to identify their own learning goals and help them see how those goals are integrated into the goals of the organization (Volpe \& Marsick, 1999). There is a growing recognition that personal fulfillment and professional fulfillment are intertwined and interdependent.

One of Senge's (1990) five disciplines, "personal mastery" refers specifically to the importance of individual growth and learning as a key component of a "learning organization". Personal mastery is defined as involving two steps: understanding what is most important to us and comprehending what is the truth. A commitment to the truth, essential in achieving the discipline of personal mastery, is not necessarily a trivial task. As Winston Churchill is purported to have said: "Sometimes we stumble over the truth but most of the time we pick ourselves up and move on" (quoted by Sterman, 2001). Those who benefit from personal mastery do not just "move on," but they stop, seeking out the evidence and the varying perspectives in order to understand what is really happing. These individuals are perpetual learners, enjoying the 'creative tension' that results from an awareness of their personal vision and how it meshes with reality. Senge explains, "the juxtaposition of the vision (what we want) and a clear picture of current reality (where we are relative to what we want) generate a creative tension"; it is the understanding of how to create and sustain this 'creative tension' that is the essence of personal mastery (p. 142).

Organizational environments conducive to informal learning recognize the importance of investing in both the personal and professional development of individuals, a critical step in enhancing the learning process. As Senge tells us, "organizations intent on building shared visions continually encourage members to develop their personal visions" (p. 211). Not surprisingly, there is true commitment to an organization's goals when the shared vision reflects one's own personal vision (Senge, 1990). Table 2, presented on the following page, provides a comprehensive picture of informal learning in the workplace. In Table 2, Volpe and Marsick (1999) identify particular characteristics of informal learning and how this type of learning can be encouraged (p. 5): 
Table 2: Informal Learning in the Workplace (Volpe \& Marsick, 1999)

\begin{tabular}{|l|l|}
\hline Informal Learning & What Enhances or Improves Learning \\
\hline Is integrated with work and daily routines & Making time and space for learning \\
\hline Is triggered by an internal or external jolt & Scan of external and internal environment \\
\hline Is not highly conscious & Heightened consciousness or awareness \\
\hline Is haphazard and influenced by change & Attention to goals and turning points \\
\hline $\begin{array}{l}\text { Is an inductive process of reflection and } \\
\text { action }\end{array}$ & Inductive mindset and reflective skills \\
\hline Is linked to the learning of others & Dependent on collaboration and trust \\
\hline
\end{tabular}

While the process of informal learning should not be controlled by the organization, a working environment to enhance informal learning can be proactively encouraged (Volpe \& Marsick, 1999). As demonstrated in Table 2, there are a variety of ways to develop an environment that is conducive to learning and to the production and dissemination of knowledge. An organization can foster informal learning by creating opportunities for its members to regularly interact with one another and work collaboratively, defining "productive" work to include "casual conversations, periods of reflection and learning" (Davenport \& Prusak, 1998, p. 93). "Slack" time should be set aside for "spontaneous, unstructured knowledge transfer" (Davenport \& Prusak, p. 89). A shared space, either electronic spaces or meeting spaces (a water cooler, for example, is listed as a prime location) can encourage individuals to informally exchange information and reflect together (Davenport \& Prusak, 1998). Mechanisms that enable knowledge sharing such as networks among colleagues, mentoring relationships, or even the practice of storytelling should be recognized or rewarded. ${ }^{18}$ In addition to enhancing informal exchange among its members, the organization needs to provide time for learning, which

\footnotetext{
18 "Learning histories", based on the tradition of community storytelling, provides an illustration of a mechanism to capture learning from experience and to circulate knowledge throughout the organization (Kleiner \& Roth, 1998). In fact, Davenport and Prusak (1998) define the knowledge of a organization as "a social construct built out of the collective stories of its workforce, the talents it rewards, and the shared stories of the firm's triumphs and mistakes" (Davenport \& Prusak, p. 64).
} 
includes time for knowledge creation and for the acquisition of new knowledge. Organizations have to be willing to value investments in learning and to reward experimentation and curiosity (see Subsection 2.1.2.2).

The type of environment that enables individuals to feel comfortable pursuing informal learning is, by its nature, characterized by a feeling of trust and collaborative spirit; individuals enjoy a "sense of community" and are rewarded for their efforts both in terms of job enrichment and personal development (Marsick, Volpe \& Watkins, 1999). While the "unstructured, experiential and noninstitutional" (Volpe \& Marsick, 1999, p. 4) nature of informal learning does not allow it to necessarily be 'managed', it can be nurtured and enhanced. In their discussion of communities of practice, Wenger, McDermott and Snyder (2002), intentionally using the word "cultivate", explain that organizations can create an environment for informal entities to prosper within the formal structure of the organization; the process is compared to the creation of a healthy environment for a plant to successfully grow on its own (p. 12). The kind of 'learning system' that successfully fosters both formal and informal learning is, by necessity, characterized by high levels of social capital. In the following section, the sixth and last characteristic, social capital, is discussed. Many of the features of social capital, such as the importance of trust, shared meaning and cooperation, further illustrate the interrelated nature of the various dimensions of a learning organization.

\subsubsection{Social Capital}

"Emergent properties," briefly addressed in the section on systems thinking, signify ways in which the behavior of the system does not represent a summation of the behavior of each part; rather "systematic behavior emerges from the interdependent activities of the parts" (Eoyang \& Berkas, 1998, p. 4). The 'systematic behavior' of a learning organization can be characterized as one that exhibits a high level of social capital. Defined as "features of social organization, such as networks, norms and trust that facilitate coordination and cooperation for mutual benefit" (Putnam, 1993, p. 1), social capital is a critical component of organizational learning (Cohen \& Prusak, 2001). The term "social capital", taking its place next to physical capital and human capital, has increasingly been recognized in recent years as contributing to (or in its absence, negatively affecting) everything from the social fabric of local communities (Putnam, 2000), the effectiveness of regional governments (Putnam, 1993), and even the economic progress of 
nations (Fukayama, 1995). Cohen and Prusak's (2001) experiential research explores the stock of social capital in organizations, reminding us that at the workplace we require "the same social needs and responses as other parts of our lives: the need for connection and cooperation, support and trust, a sense of belonging, fairness and recognition" (p. x).

In today's highly dynamic, unpredictable environment, "one needs to value relationships in order to make joint problem solving and solution implementation possible" (Schein, 1992, p. 371). Cohen and Prusak (2001) explain that "judgment, persuasiveness, shared decisions, the pooling of knowledge and the creative sparks people strike off one another all depend on engagement with the work and with one another" (p. 17). Without a minimal level of trust among members of an organization, collaborative work cannot effectively take place. Trust is "at once a precondition, an indication, a product and a benefit of social capital, as well as a direct contribution to other benefits" (p. 29). Trust in an organization is a basic, fundamental requirement for learning and for the successful exchange of knowledge (Garvin, 2000; Davenport \& Prusak, 1998). As an essential component of learning organizations, trust has been raised as an issue in earlier sections. For example, members of organizations have to trust their colleagues and leadership before they can believe that the information they bring to light will not be used against them or that they can openly talk about their lack of knowledge or mistakes without being penalized. In their discussion of the 'knowledge market', Davenport and Prusak (1998) emphasize the importance of additional factors related to trust such as reciprocity. As Putnam (2000) tells us, the concept of social capital is based on generalized reciprocity; "I'll do this for you now, in the expectation that down the road you or someone else will return the favor" (p. 1). Cooperation and knowledge sharing depend on a sense of mutuality and trust. Emphasizing the importance of a high level of trust, Schein (1992) explains that in learning organizations, "anyone must be able to communicate with anyone else and (that) everyone assumes that telling the truth as best one can is positive and desirable" (p. 370).

“Trustworthiness can only be demonstrated, not asserted" (Cohen \& Prusak, 2001, p. 45). Cohen and Prusak discuss several important ways that organizational leadership can foster an environment characterized by a high level of trust. For example, the reward and promotion system signals to members of the organization what type of behavior is valued and what kind of approaches to work are prized by the organization. An organization can ask itself how it signals to its members that knowledge sharing is rewarded more than "knowledge hoarding" (Davenport 
\& Prusak, 1998, p. 29): Are individuals who take the time to share their knowledge valued? If they collaborate jointly in a project will they receive shared credit for doing so? "Openness and trust are tightly coupled" and the organization should operate with a high level of transparency (Cohen \& Prusak, p. 47). Addressing various dimensions of transparency, Cohen and Prusak discuss how every organization member should know "what's going on" and be able to contribute and to access the knowledge base of the organization. The authors continue, "physically transparent spaces literally create organizational transparency: open access to information about what the organization is doing" (p. 91).

One of the fundamental principles of social capital is that social networks have value (Putnam, 2000). Preceding discussions on informal phenomena in organizations addressed the idea that the personal benefit to individuals (e.g. sense of belonging and identity, professional satisfaction) to, for example, a community of practice, can also enhance their learning in the workplace. Social networks, while fostering knowledge sharing and opportunities for collective learning, help to maintain organizational knowledge by cultivating a sense of loyalty among members who are consequently less likely to switch jobs (Cohen \& Prusak, 2001). The authors explain how social capital provides of sense of 'stability' and 'connection': "We all use our established personal networks and communities as tools for making sense of the world, and sense making is one of the most difficult and important activities people and organizations undertake in a changing and uncertain world" (Cohen \& Prusak, p. 153).

Putnam (1993) tells us that "stocks of social capital... tend to be self-reinforcing and cumulative" (p. 2). Likewise, it is possible to conclude that the various dimensions of a learning organization are "self-reinforcing and cumulative." Organizational investment in social capital is similar to the investment in other essential aspects of learning organizations such as the encouragement of interpersonal interaction through the support of communities and the allocation of time and space for learning. As illustrated throughout the discussion on fundamental values of learning organizations, learning organizations are cognizant of the fact that "things happen as they do (or fail to happen at all) largely because of the ways those human beings in the workplace relate to each other" (Cohen \& Prusak, 2001, p. 12). 


\subsubsection{Summarizing a Theoretical Framework for a Learning Organization}

Following this brief review of the literature on learning organizations, which draws from the fields of organizational learning, social capital, knowledge management and informal learning, six essential dimensions of learning organizations can be identified. These six dimensions of a learning organization are reviewed in Table 3 , along with accompanying actions that can help to facilitate learning in the workplace. The following section explores the critical role that evaluation can play in enhancing and facilitating the learning process.

Table 3: A Theoretical Framework for a "Learning Organization"

\begin{tabular}{|c|c|}
\hline $\begin{array}{l}\text { Dimensions of Learning } \\
\text { Organizations }\end{array}$ & Steps to Facilitate Organizational Learning \\
\hline Shared Vision/ Shared Meaning & $\begin{array}{l}\text { O Emphasizing communication } \\
\text { o Creating shared understanding } \\
\text { o Constructing meaning through storytelling } \\
\circ \text { Encouraging group dialogue and collective reflection }\end{array}$ \\
\hline Inquisitiveness and Openness & $\begin{array}{l}\text { o Willingness to question underlying assumptions } \\
\text { o Rewarding curiosity, risk taking and experimentation } \\
\text { o Nurturing a safe environment for 'failure' } \\
\text { o Learning collectively from past mistakes } \\
\text { o Scanning of internal and external environment } \\
\text { o Embracing 'dissension' and diversity of thought }\end{array}$ \\
\hline Systems Thinking & $\begin{array}{l}\text { O Adopting a long term approach to solving problems } \\
\circ \text { Recognizing patterns of change } \\
\circ \text { Addressing underlying causes of events } \\
\circ \text { Acknowledging the nature of unpredictability }\end{array}$ \\
\hline Community of Learners & $\begin{array}{l}\text { ○ Tapping into collective nature of knowledge } \\
\circ \text { Cultivating a collaborative work environment } \\
\circ \text { Understanding the importance of tacit knowledge }\end{array}$ \\
\hline Informal Learning & $\begin{array}{l}\circ \text { Redefining the definition of "productive" work } \\
\text { O Investing in personal and professional development } \\
\circ \text { Creating opportunities for interaction (providing both } \\
\text { time and space) }\end{array}$ \\
\hline Social Capital & $\begin{array}{l}\circ \text { Supporting the creation of social networks } \\
\circ \text { Ensuring that organizational policies nurture trust } \\
\circ \text { Signaling the importance of knowledge sharing and } \\
\text { importance of reciprocity }\end{array}$ \\
\hline
\end{tabular}




\subsection{EVALUATION IN ORGANIZATIONS}

In turning to the question of evaluation's role in promoting learning and adaptability in organizations, it is important to keep in mind that the field of evaluation is characterized by a range of multiple perspectives and an absence of agreement on a particular 'paradigm'. Donaldson and Scriven (2003) note that, "There is a smorgasbord of options in evaluation, some containing fundamental differences that cannot be reconciled or integrated" (p. 15). The specific focus of the study is on the role of evaluation in enhancing learning, that, as Rossman and Rallis tell us, leads to the reconceptualizing of evaluation use "as continual and collective knowledge generation and application" (Rossman \& Rallis, p. 59). In the following sections, "coevaluation" (Gray, 1998) is briefly addressed, and a specific participatory approach to evaluation, known as evaluative inquiry (Preskill \& Torres, 2000a; 1999), is examined in detail.

\subsubsection{Evaluation and Organizational Learning}

"To a large extent, a 'learning organization' is very much an 'evaluating organization"” (Davidson, 2001, p. 9). ${ }^{19}$ A learning organization, as discussed earlier, proactively collects and interprets information on a regular basis in order to inform its decision making processes. This view of evaluation, termed by Sanders (2002) as "mainstreaming evaluation", refers to the process of making evaluation an integral part of "the organization's work ethic, its culture and job responsibilities at all levels" (Sanders, 2003, p. 3). Addressing the challenge of mainstreaming evaluation, Sanders (2002) concedes the marginal role that evaluation plays in most organizations, explaining that at the present time it "is ignored, given lip service, delegated to external consultant, or left to autocratic leaders"(p. 254). In reviewing recent efforts to enhance the use of evaluation in organizations, he differentiates the goal of mainstreaming evaluation from "institutionalizing" evaluation or "building capacity in evaluation" (p. 254). The

\footnotetext{
${ }^{19}$ It should be noted that Davidson (2001) believes that it is necessary to complement "organizational development or participative inquiry intervention" (e.g., Preskill \& Torres, 1999) with "systematic evaluation from fully external (outside the organization) and semi-external (internal to the organization but external to the evaluand) sources" (p. 9).
} 
author distinguishes mainstreaming evaluation as a process that "depends in part on capacity building in evaluation, but it also depends on evaluation being internalized as a value throughout the organization and on an infrastructure that supports and maintains evaluation" (p. 254).

Co-evaluation, developed by Gray (1998), is cited by Sanders (2002) as an example of an approach that emphasizes the mainstreaming of evaluation in order to enhance organizational learning and effectiveness (Sanders, 2002). As Gray (1998) explains, the process of coevaluation, which is carried out by the organization itself on a routine basis, is characterized by three steps: "asking good questions, gathering and reviewing information and sharing the information to foster good decision making" (p. 4). Recognizing evaluation as an ongoing process that creates multiple learning opportunities, co-evaluation pushes evaluation beyond its original boundaries that have traditionally given the impression of evaluation as a 'report card' (Gray, 1998, p. xv). In the Foreword to Gray's (1998) Evaluation With Power, Patton outlines the key distinguishing features of co-evaluation and articulates how its principles differ from the traditional view of evaluations in five ways; "1. organizational effectiveness 2. evaluation is ongoing 3. evaluation as learning 4. evaluation as internal 5. evaluation as doable" (p.xii).

Co-evaluation, as Patton (1998) notes, emphasizes the use of evaluation in developing organizational effectiveness and excellence. Continuous evaluation to support learning focuses on "organizational effectiveness", with an emphasis on the assessment of the entire organization versus the assessment of a particular project or program. The second distinguishing feature of coevaluation is that "evaluation is ongoing". Whereas traditional evaluation is episodic and designed to provide summative reports at the end of the project, evaluation to support organizational learning is formative in its nature and integrated in the everyday activities of the organization $^{20}$. As Patton explains in outlining the third attribute of "evaluation as learning", traditional summative reports came to be feared by organizational members as a "singular, onetime judgment - it worked or didn't work" (p. xii). In contrast, evaluation that focuses on learning emphasizes how to improve rather than making a summative judgment of merit or

\footnotetext{
20 Summative evaluation provides a "definitive judgments about effectiveness" whereas formative evaluation focuses on "ways of improving and enhancing programs" (Patton, 1997, p. 67). In explaining the difference, Patton cites Stake (1991): "When the cook tastes the soup, that's formative, when the guests taste the soup, that's summative" (p. 69, quoted from Scriven, 1991).
} 
worth. The fourth characteristic of co-evaluation is "evaluation as internal". ${ }^{21}$ Rather than being determined by external demands for accountability, "the new vision of evaluation is that the highest form of accountability is self-accountability" (p. xii). Last, the view of "evaluation as doable" departs from the traditional social science approaches that have made evaluation "inaccessible, academic and often irrelevant" (p. xiii).

Overall, this conceptualization of evaluation as learning "simplifies the process, identifies key questions, and opens up the basic logic and potential of evaluation at a commonsensical level" (Patton, p. xiii). In further exploring the role of evaluation in promoting organizational learning, the overall framework of practical participatory evaluation is briefly reviewed, followed by discussion on the specific approach of evaluative inquiry.

\subsubsection{Evaluative Inquiry as Participatory Evaluation}

The literature on participatory evaluation, in highlighting its emphasis on fostering a learningoriented organizational culture, often addresses the role of evaluation in creating a learning organization (Patton, 1997). Evaluative inquiry, which is examined in this section, falls under the

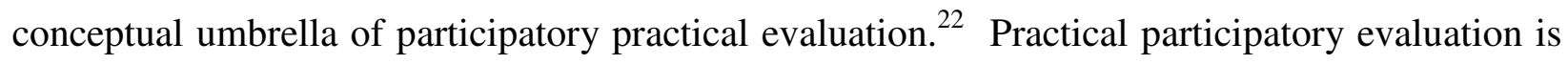
based on the belief that involvement among stakeholders in the evaluative process will increase its relevance and generate a sense of ownership among its participants, ultimately leading to the utilization of the findings (Cousins \& Earl, 1995; Cousins \& Whitmore, 1998). By engaging stakeholders in practical problem solving in their organizational settings, the "primary users" become involved in the 'nuts and bolts' of the process (Cousins \& Earl, 1995; Cousins \& Whitmore, 1998). The long term implications of the process are recognized, and the inquiry seeks to develop an organization's evaluative capacity that is self-sustaining. While providing a forum for dialogue among stakeholders (Cousins \& Earl, 1995), participatory evaluation helps to encourage staff development, a feeling of empowerment and a sense of competence with

\footnotetext{
${ }^{21}$ Throughout this discussion, there is not a distinction made between internal evaluators and evaluators who are hired as consultants to the organization. The general definition of an evaluator will be one who is "a collaborator, facilitator, interpreter, mediator, coach, and educator of learning and change processes" (Preskill \& Torres, 1999, p. 186).

${ }^{22}$ There are two streams of participatory evaluation, practical participatory evaluation and transformative evaluation (whose rationale and goals are associated with empowerment and social change). This discussion is referring to practical participatory evaluation. See Cousins \& Whitmore (1998).
} 
evaluation issues (Lafleur, 1995). Ultimately the process helps to promote organizational learning and change (Cousins \& Earl, 1995).

With its focus on evaluation practices that promote transformational learning in organizations, evaluative inquiry (Preskill \& Torres, 1999) is considered as an example of the practical participatory approach. Placing evaluative inquiry into Kirkhart's conceptual framework of evaluation use, a key objective (or 'intention' in Kirkhart's words) of the process is to facilitate learning ${ }^{23}$. With primacy placed on the process of evaluative inquiry rather than on the results, the learning itself becomes an essential outcome. The long term influence of evaluation is acknowledged and emphasized throughout the process, a perspective that aligns with Preskill and Torres's understanding of the incremental nature of learning (Kirkhart, 2000). Evaluative inquiry seeks to "mainstream" evaluation, a process that occurs when evaluation becomes a "core value" for the organization (Sanders, 2002, p. 257). In exploring evaluative inquiry, it becomes clear that this approach shares the values of 'co-evaluation'; evaluation is ongoing", 'doable', and is designed to enhance organizational effectiveness and learning by its integration into the organization's everyday activities.

\subsubsection{Rationale of Evaluative Inquiry}

Evaluative inquiry, which captures the concept of evaluation as a "catalyst for transformative learning" (p. 34), can provide an important tool for organizations that seek to become learning organizations. Preskill and Torres (2000a) believe that "learning from evaluation and from organization members' subsequent use of what they learn will most likely occur when evaluation is collaborative, is grounded in constructivist and transformational learning theories, and builds communities of practice" (p. 29). The specific term "evaluation," Preskill and Torres (2000a) point out, is not used by authors who write about constructivist and transformative learning or informal learning phenomena when referring to the process of "information gathering and problem solving." However, the authors believe that "the implications for evaluation are clear" (p. 29) and present a process of evaluation that draws on the values and principles that have been previously discussed in the section on learning organizations.

${ }^{23}$ Kirkhart's (2000) theory of evaluation use provides a framework to capture the multifaceted nature of the evaluation process. The theory has three dimensions: 1. Sources of influence (results-based and process-based); 2.Intention (intended and unintended influences of evaluation); and 3.Time (immediate versus long term impacts). 
Preskill and Torres (1999) outline four characteristics of evaluative inquiry that distinguish it from other learning-oriented evaluation approaches:

1. Evaluative inquiry is integrated into the work processes and performed primarily by organization members.

2. Evaluative inquiry is ongoing; it is not episodic or event-driven... both iterative and selfrenewing.

3. Evaluative inquiry strongly relies on the democratic process of asking questions and exploring individuals' values, beliefs, assumptions, and knowledge through dialogue and reflection.

4. Evaluative inquiry contributes to a culture of inquiry and occurring within an infrastructure that values continuous improvement and learning (pp. 184-185).

Preskill and Torres (1999) stress that the ability to successfully implement evaluative inquiry depends on the 'infrastructure' of an organization, defined as the culture, leadership, forms of communication, and systems and structures of the organization (p. 2). Understanding the 'infrastructure' and the reasons that the evaluation is being carried out is essential (Preskill \& Torres, 1999; Chelimsky, 2001). This is critical because organizations, like individuals, not only resist change, but also suffer from a particular predicament as outlined by Schon (1983); organizations can only survive when they are willing to adapt and adjust to the changing environment, while at the same time, they "have a powerful interest in the stability and predictability of organizational life" (p. 327). Chelimsky tells us that while organizations may change and use evaluation as part of the process, "it is important to understand what has triggered the change, what the change is and whether it is real or not..." (e.g., if change is driven by public accountability or public criticism; new leadership; growing competition in market, etc.) (p.17). An important objective of evaluative inquiry is to make these types of issues explicit, a necessary step for building an organizational culture that "mainstreams" evaluation.

Evaluative inquiry's three stages of 'focusing the inquiry', 'carrying out the inquiry' and 'applying learning' are accompanied by learning processes to ensure that intentions and assumptions are discussed openly. ${ }^{24}$ The four learning processes central to inquiry and integrated into each of its phases are: 1) Dialogue; 2) Reflection; 3) Asking questions and; 4) Identifying and clarifying values, beliefs, assumptions and knowledge. The structured process

\footnotetext{
${ }^{24}$ For a detailed description of the three processes, see Preskill and Torres (1999).
} 
enables those carrying out the inquiry to continually assess their actions and ensure that a shared understanding exists between the participants. For example, the team of 'inquirers' does not begin designing evaluative questions before exploring the existing motivations for learning and the desire to bring about change. As an introduction to the process, everyone is asked to answer questions such as:

- Why is it important that we develop new insights into this problem/issue at this time?

- What is the purpose of the evaluative inquiry?

- Why are these evaluative questions important?

- What do we hope will happen by answering these questions? (Preskill \& Torres, 1999, pp. 193195)

These types of questions help team members, who are drawn purposely from various departments/units of the organizations, to situate the inquiry within the larger context of the organization and gain a diversity of perspectives. Potential barriers to carrying out the inquiry and politically charged organizational issues are addressed, ultimately resulting in a more relevant and productive inquiry process. Emphasis is placed on involving all possible stakeholders and being sensitive to their varying needs for information and resources. This learning process is intentional and guided, as the design of the inquiry process ensures the existence of a 'learning strategy' (Garvin, 2000). Additional questions that enable this to take place include:

- What do we know about this problem/issue? What don't we know?

- What are the organizational variables that are affecting the context of the problem/issue?

- Why do we believe that each of these individuals or groups are stakeholders?

- How might each of these individuals or groups be affected by the outcomes of this inquiry?

- What type of data already exists that might address the evaluative inquiry questions?

- Where does the information reside? (Preskill \& Torres, 1999, pp. 193-195)

The evaluative process builds on the organization's existing 'knowledge capital' by locating where the knowledge resides and identifying learning opportunities (Preskill \& Torres, 1999). Preskill and Torres tell us that evaluative inquiry subscribes to the "philosophy of democratic accountability" and the recognition that "change starts at the individual level, with individuals taking responsibility for the collective outcomes of their own and the organization's practices" 
(p. 15). Throughout the evaluative inquiry process, the team (or 'community of inquirers') continually gauges the current level of skills, knowledge and experience, while identifying potential gaps.

The organization's receptivity to various means of communicating information is also taken into consideration. For example, during the design of the data collection phase, the team asks itself: What kind of data does the organization typically respond to? What does it ignore? (Preskill \& Torres, 1999, p. 195). Understanding that "the entry point for any learning to occur is communication of information" (p. 4), evaluative inquiry recognizes individual learning styles and the role that reflection and dialogue play in linking individual learning with organizational learning (Torres, Preskill, and Piontek, 1996). Posing various questions throughout the process facilitates dialogue and helps identify the most effective means for conveying new knowledge and insights to diverse audiences. "Social constructivist learning often happens when individuals are reviewing information together" (Preskill \& Torres, 2000a, p. 31). This process of reflection is the kind fostered in leaning organizations through informal networks such as communities of practice. As such, evaluative inquiry provides an opportunity for collective learning, emphasizing the process that takes place when members of organization are able to interpret information as a group and build communal knowledge together.

With a focus on organizational processes as well as on organizational outcomes, evaluative inquiry helps to educate and train organizational members in inquiry skills. An "evaluative attitude," where members of an organization understand the "principles and benefits of evaluation," is essential among learning organizations, and processes such as evaluative inquiry are important to help build organizational capacity (Davidson, 2001, p. 50). Involvement in the inquiry can help to spark organizational members' interest and ability to use evaluation to explore organizational issues, ultimately leading to organizational learning. As Preskill and Torres (2000a) summarize for us, "transformative learning can be facilitated when employees seek to understand something, address critical organizational issues, and improve their work through participatory, dialogic, reflective and inquiry-oriented approach to evaluation and the use of findings" (p. 29). 


\subsubsection{Evaluation as Transformational}

We know that transformational learning, a fundamental step towards becoming a learning organization, begins with the acknowledgement and examination of 'mental models'. Evaluative inquiry helps individuals uncover how their mental models or 'theory-in-use' (Argyris \& Schon, 1978) influence their thinking and actions. For example, during the data analysis and subsequent development of recommendations, the team asks itself questions such as:

- What values, beliefs, assumptions and knowledge are reflected in the choice of a particular analytic or reporting framework?

- What particular values, beliefs or assumptions are reflected in each recommendation? (Preskill \& Torres, 1999, pp.197-198)

Evaluative inquiry provides a structured framework for organizational members to explore underlying assumptions and how perceptions and actions are influenced by their values and beliefs. This is a significant step in organizational learning; otherwise transformational learning and the subsequent application of new knowledge cannot take place. As Schein (1992) cautions, "If leaders are not aware of the cultural underpinnings of what they are doing or the assumptions of the group on which they are imposing new solutions, they are likely to fail" (p. 373). Recognizing the critical role that our worldview plays in shaping the construction of knowledge, evaluative inquiry seeks to make mental models explicit through dialogue, reflection and the asking of questions.

Preskill and Torres (2000a) write that "the most significant transformative learning occurs when the purpose is to understand what others mean and to make ourselves understood" (p. 28). With an emphasis placed on creating an atmosphere of openness, the team of 'inquirers' nurtures an environment where individuals feel comfortable addressing difficult issues and voicing dissenting viewpoints. The ongoing dialogue that characterizes the process helps to brings out multiple points of view, make "hidden agendas visible", and address the "undiscussables", thereby leading to a shared understanding (Preskill \& Torres, 1999, p. 55). This shared understanding does not come instantly. "Processes that lead to transformative learning are both incremental and iterative" (Preskill \& Torres, 2000a, p. 30). Evaluative inquiry takes place over time as it continually incorporates past learning and experiences while allowing the opportunity to integrate new insights. 


\subsubsection{Evaluation as Dynamic}

Eoyang and Berkas (1998) tell us that evaluations have traditionally been based on the assumption that the organization under assessment is "closed, stable and predictable" (p. 1). Yet, as they explain, for the evaluation to be effective, it "must match the dynamics of the system to which it is applied" (p. 1). Systems thinking requires a holistic approach that recognizes, for example, the existence of feedback loops and the interdependency of different organizational components (see Subsection 2.1.2.3). Adopting a systems perspective, evaluative inquiry does not assume that any outcome is predictable, and continually reassesses the process to meet the changing reality. Examples of questions that can foster this type of thinking during the data design, collection and interpretation include:

- What type of data will indicate if the activities, procedures, or policies in question are being implemented as intended or will inform us of any unanticipated outcomes?

- How is the organization reacting to carrying out the inquiry?

- What adjustments to the data collection plan need to be made?

And, for example, in the consideration of action alternatives:

- To what extent will the organization's existing infrastructure either support or undermine implementation of this action alternative?

- Conversely, what impact will it likely have on any elements of the infrastructure? (Preskill \& Torres, 1999, pp. 195-199)

Organizations, as complex systems, are characterized by interconnectedness and unpredictability (Eoyang \& Berkas, 1998, p. 1). Recognizing the unpredictable nature of change and the potential impact of the evaluative process on the organization, evaluative inquiry helps the 'inquirers' to anticipate possible consequences or outcomes. Schein (1992) tells us that "the optimal time orientation for learning appears to be somewhere between far future and near future. One must think far enough ahead to be able to assess the systemic consequences of different courses of action, but one must also think in terms of the near future to assess whether or not one's solutions are working" (p. 369). Evaluative inquiry seems to achieve this balance. Building on the past experiences of the organizations, it recognizes the importance of looking towards the future while continually evaluating the current actions of the organization. 


\subsubsection{Evaluation as Action}

In the earlier discussion on informal learning, the notion that individuals learn new ideas most effectively when they are "linked to everyday challenges" was raised (Garvin, 2000, p. 117). In learning organizations, knowledge production and dissemination take place as part of the everyday work, and the learning process is iterative and ongoing. Recalling the writings of John Dewey, Garvin (2000) stresses the link between learning and experience; the application of new knowledge needs to be accompanied by "a process that includes pauses along the way to evaluate progress, share leanings, and make midcourse corrections" (p. 117). Evaluative inquiry interweaves the learning process with ongoing dialogue and action that feeds back into the process itself, resulting in continuous learning. By doing so, the process helps to overcome the problem of "inertia" and "passivity" that characterizes the last stage of the learning process, the application of information (Garvin, 2000). Upon completing the first two steps of the learning process - the acquisition and interpretation of information - the final step of applying information is not ignored. Team members design specific actions that are monitored and continually reassessed to see if the implementation is taking place as planned. The 'theory of action' helps to translate the new knowledge into specific organizational practices and accomplishments; the 'vision' turns into an action plan with questions such as "What does it look like? How will it feel? How do we get there? (p. 163). The 'theory of action' underlying the process is continually reassessed throughout the evaluative inquiry.

Using the 'theory of action' as its framework, the process of evaluative inquiry helps to sheds light on potential gaps between the program's 'espoused theory' and its 'theory-in-use' (Preskill \& Torres, 2000a). In practice, we modify our espoused theory, either consciously or unconsciously, and employ our 'theory-in-use' (Argyris \& Schon, 1996). It is critical to recognize the gap between our espoused theory (what we say) and what we do, for learning is not just about new ideas or information but about translating these new insights into new behavior (Zeigler, 1999). The process of evaluative inquiry consciously seeks to bring out their 'alignment' (Preskill \& Torres, 2000a). Questions such as the following facilitate this process:

- How will we know if the action is being implemented successfully?

- How is actual implementation paralleling intended implementation and what can we learn from any discrepancies? (Preskill \& Torres, 2000a, pp. 199-200) 


\subsubsection{Evaluation as Routine}

In the preceding discussion on learning organizations, several authors emphasized the importance of allocating time and space for learning, including time for dialogue and reflection. Using the U.S. Army as an example, Garvin (2000) discusses its success in integrating reflection and review processes to the extent that it has become "second nature" (p. 115). An organizational commitment to factors such as openness, broad participation and the availability of objective data help to ensure that the process is productive and valued by participants. Perhaps most important, Garvin explains, is the notion that repetition allowed the process to become routine ("consistency breeds comfort and acceptance", p. 115). He describes the process as follows: "A new mindset develops in the organization, a recognition that no activity is truly complete until participants have reflected on their experiences and understood the reasons for success or failure. Then and only then, has learning been incorporated into daily work" (p. 115).

Evaluative inquiry's overall structure builds in time for reflection and dialogue throughout all phases of the learning process. The question of whether or not to make time for learning no longer is relevant because it is considered part of how work is done (Preskill \& Torres, 2000a). Evaluative inquiry's conceptual framework and the 'naming' of the process grants a legitimacy to its activities and "encourages busy people to give priority to the time that organizational learning requires" (p. 32)

In evaluative inquiry, the process of evaluation has built-in steps to facilitate learning. The next section considers the possibility of monitoring the 'process of learning' and of measuring an organization's learning culture. Efforts to evaluate the current status of an organization and its capacity for learning is explored in brief.

\subsection{PRACTICAL METHODS OF ASSESSMENT}

As tempting as it may be, Ziegler (1999) cautions us that one can never really ask the question, "Are we there yet?"(p. 64). "There is no there" when it comes to building learning organizations (Senge, p. xv). Ziegler (1999) reminds us that with transformational learning, "a destination or goal will always by necessity be changing and thus remain beyond reach" (p. 64). Keeping in mind that change will not be revolutionary - not "one big discontinuous, shattering break" 
(Collins, 2002), efforts can be made to measure this type of gradual and ongoing change. Continual assessment allows one to see if the learning is becoming part of the organization's theory-in-use and "embedded in the organizational environment - its epistemological artifacts (the maps, memories, and programs)" (Argyris \& Schon, 1996, p. 16).

While acknowledging the inherent difficulty in seeking to measure progress of this type of change and the "fuzzy edges" of organization learning (Argyris \& Schon, 1996, p. 16), it is possible to consider the significance of assessment in the process of change and review available tools for helping an organization to identify if it enjoys a learning-oriented culture or a "learningimpaired culture" (Davidson, 2001). Understanding the organizational work environment and the willingness of its members to engage in change efforts and evaluative processes is considered as an essential first step in moving towards becoming an organization with a high 'learning capacity' (Davidson, 2001). Several, brief litmus tests designed by Garvin (2000) are examined in the

following section. A review of three assessment tools designed to provide insight into the learning culture of an organization (Preskill \& Torres, 2000b; Davidson, 2001; Watkins \& Marsick, 1999) are then discussed in detail.

\subsubsection{Litmus Tests}

As discussed earlier, there are certain characteristics that foster an environment conducive to learning and are therefore present in learning organizations. Garvin (2000) designed a few litmus tests for organizations that are looking for "obvious clues" as to whether they are acting as a learning organization (p. 13). The five questions are the following:

1. Does the organization have a "defined learning agenda?"

2. Is the organization "open to discordant information?"

3. Does the organization "avoid repeated mistakes?"

4. Does the organization "lose critical knowledge when key people leave?"

5. Does the organization "act on what it knows?" 
We can briefly review each of the five questions:

\section{Does the organization have a "defined learning agenda?"}

Does the organization have an awareness of what it needs to know? Is it clear what knowledge is needed to successfully pursue the organization's strategic goals? The organization can consider if it explicitly acknowledges and encourages knowledge-sharing activities. It should also ask itself if gaps in knowledge are identified and the organizational approach to achieving this knowledge is articulated. Does the organization fall into the trap of underestimating its knowledge and informational needs? Do they keep in mind that "it is what we think we know already that often prevents us from learning" (Claude Bernard, quoted in Raelin, p. 27)?

\section{Is the organization "open to discordant information?"}

In posing this question, Garvin asks if organizations are willing to hear the unwanted news or if they "shoot the messenger," an indication that the organization is hostile to learning (Garvin, p. 13). Is the leadership prepared to listen to information that may contradict current practices or beliefs? Is there a readiness to ask questions and challenge the status quo? Is knowledge that is held viewed as provisional (Garvin, 2000)? The way in which inquiry and openness to diversity of opinion are perceived in the organization constitutes a strong indicator of whether learning will take place. Is the asking of questions that explore underlying assumptions in the decision making process encouraged or is that seen as evidence of a "challenge, threat, or insubordination" (Bickel, Millet, \& Nelson, p. 3, 2002)? In a learning environment, trust allows individuals to feel comfortable speaking openly and truthfully; there is a shared understanding that it is more important to ask the hard questions than to avoid controversy. Openness to contradictory information ultimately results in deeper learning and "conflict becomes, in effect, part of the ongoing dialogue" (Senge, 1990, p. 249).

\section{Does the organization "avoid repeated mistakes?"}

Great organizations are characterized by "how quickly bad news travels upward" (Forrester, quoted in Senge, 1990, p. 226). A learning-oriented culture will enable individuals to feel

comfortable discussing mistakes (see Subsection 2.1.2.2). Without a psychologically safe 
environment, problems will not be identified and addressed; "fear does little to encourage learning" (Garvin, p. 41). Acknowledged as a necessary part of experimentation and innovation, error is tolerated by organizations who strive to facilitate the learning process. An approach to learning that recognizes the importance of sustaining creative tension, as discussed earlier in the context of "personal mastery" (Senge, 1990), transforms the perception of failure; "Failure is, simply a shortfall, evidence of the gap between vision and current reality" (Senge, p. 154). Mistakes and failures are considered as opportunities for learning; an approach that is captured by a quote that hung on the wall of the company Polaroid's founder: "A mistake is an event, the full benefit of which has not yet been turned to your advantage" (quoted in Senge, p. 154).

\section{Does the organization "lose critical knowledge when key people leave?"}

In other words, how often does knowledge "walk out the door?" (Davenport \& Prusak, 1998, p. 44). Organizations have to create the time and space for their members to exchange knowledge, learn collectively and work in cooperation. Only through the sharing of knowledge can knowledge become part of the organization's "values and norms and operating practices", and ultimately, the "common property" of the organization (Garvin, p. 15). Learning organizations ensure that both tacit and explicit knowledge is 'institutionalized' through a variety of formal and informal mechanisms.

\section{Does the organization "act on what it knows"?}

Does the organization "know what it knows" 25 and use this knowledge accordingly? In posing this final question to organizations, Garvin (2000) addresses the difficulty of knowledge application. This "inability or unwillingness to act on new interpretations" (Garvin, 2000, p. 33) raises the issue of a gap between 'espoused theories' versus 'theories-in-use'. Translating a changed assumption into action is difficult, but without changed behavior, is it possible to conclude that we have learned? As Ziegler tells us, "The inability to convert new convictions into practice is a critical juncture in the learning process." (p. 62). She continues, "It is possible to be exposed to new ideas, to enthusiastically adopt new ideas, and still be unable to apply new ideas" (p. 63). Despite the difficulty of judging the value of new knowledge and making the link

\footnotetext{
${ }^{25}$ This is a rephrasing of Hewlett-Packard CEO Lew Platt' statement "if HP knew what HP knows, we would be three times as profitable" (quoted in Davenport \& Prusak, 1998, p. xxi).
} 
between knowledge and decision making, "knowledge can and should be evaluated by the decisions or actions to which it leads" (Davenport \& Prusak, 1998, p. 6).

Garvin (2000) tell us that the existence of the traits outlined in the litmus tests does not guarantee a learning organization; however, "their absence certainly raises grave doubts" (p. 13). If the organization does not have a learning agenda, is not open to discordant information, does not avoid repeated mistakes, loses critical information in turnover, and does not "act on what it knows," it is safe to assume that it is not heading in the direction of becoming a learning organization. Assessment surveys, the topic of the next section, are considered as an important tool for organizations who are interested in systematically checking if they are heading in the right direction. Assessment is essential for both determining the level of organizational readiness for initiating change efforts and acting as guide throughout the process.

\subsubsection{Assessment Tools}

In gaining a sense of whether the organization enjoys a high or low level of social capital, a critical element in learning organizations, Cohen and Prusak (2001) discuss the "feel" of an organization. Gaining insight into the "feel" of an organization can help provide an understanding of the organization's daily reality that go beyond the picture of an organization that is portrayed in its formal literature, mission statements or organizational charts (Cohen \& Prusak, 2001). Assessment tools, based on the responses of organizational members, can grant access to the day-to-day realities of organizational life and provide a window into the organizational culture. Three assessment tools developed by Watkins and Marsick (1999), Preskill and Torres (2000b) and Davidson (2001) are briefly profiled in this following section. All three tools seek to assess the 'learning capacity' of an organization - defined by Davidson (2001) as "the extent to which the organization's culture creates a work environment that is conducive to organizational learning and effectiveness" (p. 96). 


\subsubsection{Dimensions of the Learning Organization Questionnaire (DLOQ)}

The Dimensions of the Learning Organization Questionnaire (DLOQ), developed by Watkins and Marsick (1999), can help organizations to gain a greater insight into their current condition as an organization and to more effectively manage the change process. The DLOQ, drawn from the authors' own research and practice in the field of learning organizations and human resource management, is designed both as a diagnostic tool and as a comparative measurement among organizations (Marsick and Watkins, 2003). Examining the organization's 'climate, culture, systems and structures', the DLOQ is based specifically on seven dimensions of learning organizations:

1. Creation of continuous learning opportunities

2. Promotion of inquiry and dialogue

3. Encouragement of collaboration and team learning

4. Creation of systems to capture and share learning

5. Empowerment of people toward a collective vision

6. Connection of the organization to its environment

7. Provision of strategic leadership for learning (Marsick and Watkins, 2003)

The DLOQ is divided into four distinct sections: individual learning, team learning, organizational learning, knowledge performance and financial performance. In its first three sections, the DLOQ questionnaire asks its respondents to consider how their organization supports and uses learning at an individual, team, and organizational level. The last section is designed to help organizations examine the connection between organizational learning and organizational performance (Watkins \& Marsick, 2003). Respondents are therefore asked questions related to the knowledge performance (or level of 'knowledge capital') and the current financial performance of their organization (i.e., In my organization, the number of individuals learning new skills is greater than last year, and In my organization, return on investment is greater than last year) (p. 145).

The DLOQ is accompanied by a Participant's Guide for Interpreting Results of DLOQ (O'Neil, 2003) which helps an organization to interpret the questionnaire results, identify its organizational strengths and pinpoint the areas of greatest strategic leverage to become a learning organization. In the Participant's Guide, the sections on individual, group and organizational learning are consequently divided into subsections based specifically on the seven dimensions of learning organizations listed above. In accordance to the Participant's Guide, Table 4 illustrates 
how the categories are divided for the purpose of analysis, and lists sample questions for each section (respondents answer on a 1-6 point scale ranging from "almost never" to "almost always"). The results of the three sections that are briefly reviewed in Table 4 are then compared to the two organizational performance measurements (knowledge and finance performance), that are self-reported in the final section of DLOQ.

Table 4: Dimensions of the Learning Organization Questionnaire (DLOQ)

\section{The Individual Level}

Creating continuous learning opportunities (e.g.):

- In my organization, people openly discuss mistakes in order to learn from them.

○ In my organization, people identify skills they need for future work tasks.

Promotion of Inquiry and Dialogue (e.g.):

○ In my organization, people give open and honest feedback to each other.

- In my organization, people spend time building trust with each other.

\section{The Team or Group Level}

Encouraging collaboration and team learning (e.g.):

○ In my organization, teams/groups have the freedom to adapt their goals as needed.

- In my organization, teams/groups are rewarded for their achievements as a team/group.

\section{The Organizational Level}

Creating systems to capture and share learning (e.g.):

○ My organization enables people to get needed information at any time quickly and easily.

- My organization makes its lessons learned available to all employees.

Empower people toward a collective vision (e.g.):

- My organization invites people to contribute to the organization's vision.

- My organization gives people control over the resources they need to accomplish their work.

Connect the organization to its environment (e.g.):

- My organization encourages everybody to bring the customers views into the decisionmaking process.

○ My organization encourages people to get answers from across the organization when solving problems. 
Table 4: Dimensions of the Learning Organization Questionnaire (DLOQ), continued

Provide strategic leadership for learning (e.g.):

- In my organization, leaders share up-to-date information with employees about competitors, industry trends and organizational directions.

○ In my organization, leaders ensure that the organization's actions are consistent with its values.

As a tool to measure the perceptions of organizational members towards their organization's capacity for learning, the DLOQ offers organizations the opportunity, with the help of the Participants' Guide, to improve their ability to assess and eventually enhance their learning environment (O'Neil, 2003). Each question of the DLOQ is interpreted for the organization in the Participants' Guide. For example, if an organization scores low on the question: in my organization, people help each other learn, the Participants' Guide suggests that the organization consider whether individual success is encouraged at the expense of investment in the organization's collective knowledge. The Participant's' Guide then recommends strategies to encourage collective learning, such as providing greater rewards for team and organizational achievement and recognizing employees who serve as mentors and help others learn. ${ }^{26}$

The DLOQ is based on self-reporting of employees, and, as Marsick and Watkins (2003) stress, it is "just a snapshot of perceptions of change at the time the instrument is taken" (p. 138). ${ }^{27}$ The authors recognize limitations associated with self-reporting, emphasizing that it can be particularly problematic in the measurement of the knowledge and financial performance indicators (See Marsick \& Watkins, 2003). While keeping in mind the limitations of DLOQ, Marsick and Watkins identify patterns that they have found in the results of the DLOQ. The information gathered from the over two hundred organizations that have taken the DLOQ point to a correlation between learning organization dimensions and knowledge and financial performance. ${ }^{28}$ The authors note that tools such as DLOQ can help organizations understand the importance of having systems in place to promote learning and to allow for the scanning of both the organization's internal and external environment.

\footnotetext{
${ }^{26}$ For additional examples, see Participants Guide to Interpreting Results on the DLOQ (O'Neil, 2003)

${ }^{27}$ For more information on the validity of the instrument, see Marsick \& Watkins (2003) p. 136.

${ }^{28}$ For further about the research findings, see Marsick \& Watkins (2003) p. 136.
} 


\subsubsection{The Readiness for Organizational Learning and Evaluation Instrument (ROLE)}

Based on their conceptualization of evaluative inquiry (Preskill \& Torres, 1999), Preskill and Torres (2000b) have designed an assessment tool that can be used to measure organizational readiness to carry out participatory evaluation and foster organizational learning. ${ }^{29}$ Similar to DLOQ, the Readiness for Organizational Learning and Evaluation Instrument (ROLE) is designed to help an organization assess its own learning culture as well as to continue tracking its progress over time. Yet, ROLE places much greater emphasis on the importance of evaluation and, in contrast to the DLOQ, does not explore a correlation between learning organization dimensions and outcome measurements. As experts in the field of evaluation, Preskill and Torres stress the role of evaluation in the organization learning process and design their assessment instrument accordingly. The ROLE questionnaire is based on their belief that certain elements of an organization's 'infrastructure' need to exist in order to support organizational learning and encourage the process of evaluative inquiry. The questionnaire consequently is divided into six sections. The first four dimensions of the questionnaire measure the organization's 'infrastructure' (culture, leadership, forms of communication and systems and structures), while the last two sections are teams and evaluation.

The ROLE questionnaire is designed to help organizations determine the extent to which there are practices and systems in place to support evaluation and organizational learning. Understanding the importance of organizational support for carrying out evaluative efforts, Preskill and Torres (2000b) have developed an organizational assessment based on the opinions and experience of employees that examines the capacity for learning from evaluative inquiry. As illustrated in Table 5 on the following page, each of the six aspects of organizational readiness is divided into subsections and has sample questions. Respondents are asked to respond to a Likert scale item on a scale of 1 to 5, with 1 meaning "strongly disagree", and 5 meaning "strongly agree".

\footnotetext{
${ }^{29}$ The questionnaire and a brief explanation of the instrument was published as an appendix in Russ-Eft and Preskill (2001).
} 
Table 5: Readiness for Organizational Learning and Evaluation Instrument (ROLE)

\section{Culture}

Collaboration and Problem Solving (e.g.):

○ Employees often stop to talk about the pressing work issues they're facing.

- When trying to solve problems, employees use a process of working through the problem before identifying solutions.

- Employees operate from a spirit of cooperation, rather than competition.

Risk Taking (e.g.):

- Employees are committed to being innovative and forward looking.

- Employees are confident that mistakes or failures will not affect them negatively.

Participatory Decision Making.

- Asking questions and raising issues about work is encouraged.

- I feel safe explaining to others why I think or feel the way I do about an issue.

○ Employees are encouraged to offer dissenting opinions and alternatives.

\section{Leadership (e.g.)}

- Managers and supervisors admit when they don't know the answer to a question.

- Managers and supervisors model the importance of learning through their own efforts to learn.

- Managers and supervisors are open to negative feedback from employees.

\section{Systems and Structures (e.g.)}

Open and accessible work environment

- There is little bureaucratic red tape when trying to do something new or different.

- Workspaces are designed to allow for easy and frequent communication with each other.

- There are few boundaries between departments/units that keep employees from working together.

\section{Systems and Structures (e.g.)}

Rewards, and recognition system and practices

- The current reward or appraisal system recognizes, in some way, team leaning and performance.

○ Employees are recognized or rewarded for helping each other learn.

○ Employees are recognized or rewarded for experimenting with new ideas.

Relationship of work to organizational goals

- Employees understand how their work relates to the goals or mission of the organizations.

- Employees performance goals are clearly aligned with the organization's strategic goals. 
Table 5: Readiness for Organizational Learning and Evaluation Instrument (ROLE), continued

\section{Communication of Information (e.g.)}

- Information is gathered from clients, customers, suppliers or other stakeholders to gauge how well we're doing.

○ There are systems to manage and disseminate information for those who need and can use it.

5. Teams (e.g.) (note: if respondent answers that teams or working groups exist in organization)

○ Team members address both team processes and work content.

○ Team meetings strive to include everyone's opinion.

- Teams are an effective way to meet an organization's goal.

\section{Evaluation (e.g.)}

- The integration of evaluation activities into our work has enhanced (or would enhance) the quality of decision-making.

- There are evaluation processes in place that enable employees to review how well changes we make are working.

The results of the ROLE questionnaire can serve to identify existing characteristics of learning organizations, gauge the level of interest in evaluative inquiry, and help an organization to appropriately design evaluation efforts to support organizational learning (Preskill \& Torres, $2000 b){ }^{30}$

\subsubsection{3 "Learning Capacity" Assessment}

The third and final assessment tool to be reviewed is a questionnaire designed by Davidson (2001) to evaluate an organization's overall ability to facilitate learning. As an evaluation expert, Davidson developed the assessment tool as part of a comprehensive methodology for measuring learning capacity. What distinguishes Davidson's work from ROLE and DLOQ is her effort to convert the results of the questionnaire into an "explicit determination of merit" (p. 85). ${ }^{31}$ Whereas ROLE and DLOQ were primarily developed to help organizations assess their readiness

\footnotetext{
${ }^{30}$ To the author's knowledge, there is not additional research published on ROLE. An assessment scale based on Preskill \& Torres (1999) is discussed in Botcheva, White \& Huffman (2002).

${ }^{31}$ It is beyond the scope of this paper to explore this issue more in detail: see Davidson (2001).
} 
for organizational learning and to guide the change process, Davidson's learning capacity assessment is not designed as a self-administered instrument. ${ }^{32}$

Based on her theoretical framework of organizational learning, Davidson's questionnaire is structured around eight cultural dimensions of organizational learning capacity. These selected dimensions form the 'criteria' that are subsequently used for evaluating organizational learning capacity. The first five dimensions are drawn from Senge's (1990) five disciplines of a learning organization (personal mastery, mental models, shared vision, team learning and systems thinking) and three additional dimensions are added (scanning, experimentation and evaluation). In Table 6, the eight dimensions of learning culture are listed along with the sub-definitions for each dimension and sample questions for each section. ${ }^{33}$

Table 6: Organizational 'Learning Capacity' Assessment

\begin{tabular}{|c|c|c|}
\hline $\begin{array}{l}\text { Learning Culture } \\
\text { Characteristic }\end{array}$ & Sub-Definitions & Examples of Survey Questions per category \\
\hline $\begin{array}{l}\text { 1. Personal } \\
\text { mastery }\end{array}$ & $\begin{array}{l}\text { Pursuit of inspiring } \\
\text { goals, truth about } \\
\text { one's own } \\
\text { performance, and } \\
\text { shared understanding } \\
\text { of gap between } \\
\text { current and desired } \\
\text { performance. }\end{array}$ & $\begin{array}{l}\text { O This organization effectively supports people with } \\
\text { ambitious ideas for improvement. } \\
\text { o People here are resistant to having their work critiqued } \\
\text { by someone else. } \\
\text { o People here have an accurate sense of where the } \\
\text { organization's performance falls short of excellence. } \\
\text { O The people I work with have very different opinions } \\
\text { about where our organization needs to improve. (reverse } \\
\text { coded) }\end{array}$ \\
\hline
\end{tabular}

\footnotetext{
${ }^{32}$ To note again, Davidson believes that it is necessary to complement "organizational development or participative inquiry intervention" (e.g. Preskill \& Torres, 1999) with "systematic evaluation from fully external (outside the organization) and semi-external (internal to the organization but external to the evaluand) sources" (p. 9). Her overall methodology therefore combines elements of internal, semi-internal and external evaluation.

${ }^{33}$ Davidson (2001) focuses on organizational culture versus organizational performance in order to (a) avoid the complexity of attributing causal attributions for performance, (b) provide time for identifying potential problems in order to affect change, and, (c) present an accurate indication of the current ( as opposed to the previous) state of the organizational 'health' (p. 58).
} 
Table 6: Organizational 'Learning Capacity' Assessment, continued

\begin{tabular}{|c|c|c|}
\hline 2. Mental Models & $\begin{array}{l}\text { Diversity of thought, } \\
\text { no "sacred cow," } \\
\text { strong sense of trust. }\end{array}$ & $\begin{array}{l}\text { In this organization, we pay serious attention to the } \\
\text { opinions of people who think differently from everyone } \\
\text { else. } \\
\text { o Newcomers to this organization soon learn that there are } \\
\text { some policies and practices that it is not safe to suggest } \\
\text { changes in. } \\
\text { Anything negative you say around here is likely to have } \\
\text { consequences later on. (reverse coded) }\end{array}$ \\
\hline 3. Shared Vision & $\begin{array}{l}\text { Shared vision, sense } \\
\text { of community and } \\
\text { shared identity, long } \\
\text { term commitment to } \\
\text { organization, people } \\
\text { use own judgment } \\
\text { and common sense } \\
\text { in their work. }\end{array}$ & $\begin{array}{l}\text { I really feel like my work makes a difference in people's } \\
\text { lives. } \\
\text { o People here are deeply committed to the long -term } \\
\text { survival of this organization as a work community. } \\
\text { O Many performance problems here are caused by a lack } \\
\text { of clarity about how the job should be done. (reverse } \\
\text { coded) }\end{array}$ \\
\hline 4. Team Learning & $\begin{array}{l}\text { Team synergy, true } \\
\text { dialogue and good } \\
\text { cross-project } \\
\text { communication. }\end{array}$ & $\begin{array}{l}\text { O When we work together in groups, we often achieve a } \\
\text { 'synergy' that makes us more productive than we would } \\
\text { be as individuals working independently. } \\
\text { o We have fruitful, constructive debates about new ideas. }\end{array}$ \\
\hline $\begin{array}{l}\text { 5. Systems } \\
\text { Thinking }\end{array}$ & $\begin{array}{l}\text { Understanding } \\
\text { interdependence of } \\
\text { parts of organization, } \\
\text { seeking out } \\
\text { systematic causes of } \\
\text { problems and causal } \\
\text { loops. }\end{array}$ & $\begin{array}{l}\text { People hear are very conscious of how changes in one } \\
\text { part of the organization can affect other parts. } \\
\text { When faced with problems, we usually only look for } \\
\text { causes in the obvious places. (reverse coded) } \\
\text { When we have a recurring problem, we look critically at } \\
\text { our processes/systems in order to find where the real } \\
\text { cause lies. }\end{array}$ \\
\hline $\begin{array}{l}\text { 6. External and } \\
\text { Future Scanning }\end{array}$ & $\begin{array}{l}\text { Awareness of the } \\
\text { external environment } \\
\text { and possible changes } \\
\text { in the future, change } \\
\text { considered as } \\
\text { positive and natural. }\end{array}$ & $\begin{array}{l}\text { We are highly sensitive to our external environment, } \\
\text { enabling us to pick up cues about trends and emerging } \\
\text { opportunities. } \\
\text { We seem to spend most of our time on day-to-day } \\
\text { matters, leaving little or no time to think about the } \\
\text { future. (reverse coded) } \\
\text { Most attempts to change things are greeted with } \\
\text { cynicism and resistance. (reverse coded) }\end{array}$ \\
\hline
\end{tabular}


Table 6: Evaluating Organizational Learning Capacity, continued

\begin{tabular}{|c|c|c|}
\hline 7. Experimentation & $\begin{array}{l}\text { Support for risk- } \\
\text { taking, diversity of } \\
\text { practice, } \\
\text { marketplace for } \\
\text { ideas, and } \\
\text { streamlining and } \\
\text { constant } \\
\text { improvement. }\end{array}$ & $\begin{array}{l}\text { O The real 'heroes' in this organization are the ones who } \\
\text { 'stuck their necks out' and took a few calculated risks. } \\
\text { o Even when the accepted method is not optimal, everyone } \\
\text { here still does things in much of the same way. (reverse } \\
\text { coded) } \\
\text { O I often see people in this organization ignore very good } \\
\text { ideas. (reverse coded) } \\
\text { We have excellent new ideas coming from all levels of } \\
\text { the organization. } \\
\text { When developing something new, we tend to make a } \\
\text { detailed plan, and then stick to it quite diligently. } \\
\text { (reverse coded) }\end{array}$ \\
\hline $\begin{array}{l}\text { 8. Systematic } \\
\text { Evaluation }\end{array}$ & $\begin{array}{l}\text { Value focus of } \\
\text { personnel } \\
\text { evaluation, flexible } \\
\text { use of goals, use of } \\
\text { multiple } \\
\text { perspectives, } \\
\text { understanding of } \\
\text { customer. }\end{array}$ & $\begin{array}{l}\text { The way peoples' performance is evaluated accurately } \\
\text { reflects the value they add to the organization. } \\
\text { o In this organization, there is incentive for us to set our } \\
\text { goals low enough to make sure they can be easily } \\
\text { achieved. (reverse coded) }\end{array}$ \\
\hline
\end{tabular}

In an effort to explore the potential connection between learning culture and outcome measures, respondents are also asked in the last two sections to answer several questions related to the variables of individual performance and employee retention. For the first outcome variable, respondents are asked to consider their individual performance and whether their work environment enhances their ability to contribute, rating the "extent to which you are able to impact the performance of the company" (p. 97). Questions on retention, the second outcome variable, are designed to explore the level of organizational commitment (e.g. "I feel a strong sense of loyalty to this organization") and intent to stay with the organization (p. 189). ${ }^{34}$

In an effort to provide an overall evaluative conclusion for each of the three participating companies in her study, Davidson (2001) interviewed the owners of the companies, administered

\footnotetext{
${ }^{34}$ Citing (Senge, 1990), Davidson (2001) highlights the notion that learning organizations provide more fulfilling professional and personal work environments, and therefore retention rates can be an important indicator.
} 
the 'learning capacity' questionnaire to employees and analyzed organizational documentation. Her research findings point to the importance of systematic evaluation and its link to organizational success. As Davidson explains, the findings support the idea that a "learning organization" is, in many ways, an "evaluating organization." As a "key leverage point" for enhancing organizational learning, the use of evaluation is essential in order to become a "metalearning" organization, defined as "those that continually evaluate and improve their own learning capabilities" (p. 7). ${ }^{35}$

The preceding discussions on learning organizations, evaluation, and practical assessment begin to shed light on the complex nature of organizational learning and the significant challenges involved in facilitating the ability of an organization to enjoy a learning-oriented culture versus a "learning-impaired culture" (Davidson, 2001). At this stage it is time to take the general discussion regarding organizations and apply it to the specific circumstances found among nonprofit organizations. In the following section, the unique conditions facing nonprofits as organizations are considered, as well as the role of capacity building in enhancing nonprofits' ability to increase their social impact.

\subsection{THE NONPROFIT CONTEXT}

To provide some insight into the particular context of this study, a very brief overview of the nonprofit sector in Israel is presented. This is followed by a discussion of the overall culture of nonprofit organizations and philanthropic institutions, a culture that has traditionally deemphasized the importance of strengthening organizational capacity. A framework for addressing organizational capacity building for nonprofits is outlined, and more specifically, the meaning of adaptive capacity is then defined. Lastly, the role of organizational readiness for capacity-building efforts is explored.

\footnotetext{
${ }^{35}$ Davidson (2001) discusses the lack of empirical research testing the link between Senge's (1990) five disciplines with organizational outcomes. She raises the question of whether certain disciplines affect organizational effectiveness differently or vary in importance depending on, for example, organizational size, organizational context or the type of industry (p. 12). For more information on her study, see Davidson (2001).
} 


\subsubsection{The Nonprofit Sector in Israel}

Historically, the centralistic nature of governing and the bureaucratic institutions established after 1948 in Israel served the needs of a society undergoing constant pressures of security, economic development, immigration absorption and nation-building. In the early decades of the state, the "bureaucratic hierarchical decision-making structure" (Gidron, 1992) of the Israeli governing institutions existed in the midst of a political climate that emphasized the need for national solidarity and the creation of a national identity (Eisenstadt, 1985). A comprehensive welfare system was developed to further the national commitment to social engineering and equity (Doron, 1985; Cnaan, 1987). Throughout the 1950's, 1960's and early 1970's, the social, economic and political institutions historically discouraged the autonomic participation of citizens in independent action that was not within the framework of the electoral system (Lazin, 1996).

The centralistic nature of the government in Israel began to decline by the 1970's, resulting in a less monolithic system of decision making, a downsizing of the welfare state and a shift in the relationship between the government and its citizens. The growing awareness of the tool of independent collective action and citizen participation is a significant outcome of this process (Gidron, 1992). Indeed, from 1973 to 2005, the number of nonprofits organizations that are registered in Israel grew from 1,000 to 30,000, representing an increase that is considered especially dramatic for a country of six million people (Reardon, 2005). While the majority are providing some type of direct services, approximately one in ten are social change organizations are addressing economic and social justice issues (Reardon, 2005). "Israelis have begun to recognize that change does not just occur through political parties. They see that civil society is now a major force for developing solutions to collective problems and that citizens can bring about social change if they organize" (Liel, quoted in Reardon, 2005). During this period, marked societal trend such as the growing influence of the mass media (Eisenstadt, 1985) and the increased level of citizen participation in the public sphere (Alterman, 1995) accompanied the extensive growth in number and influence of nonprofit organizations in Israel, especially those in 
the field of advocacy and social change. ${ }^{36}$ Major historical events during this period of growth that impacted Israeli society, and more specifically, the development of social change organizations, include the first Palestinian Intifada (Uprising) in 1989-1991, the mass immigration from the Former Soviet Union starting in 1991 (eventually reaching 1 million immigrants) and immigration of over 40,000 from Ethiopia in the 1980 and 1990s, the Oslo peace treaty in 1993, the assassination of Prime Mister Rabin in 1995 and the second Intifada starting in 2000 .

Due to the socio-political nature of Israel, and especially in light of the violence in recent years, Israeli nonprofits play an increasingly critical role in working to strengthen the country's democracy. These social change nonprofits (addressing issues such as civil and human rights, religious tolerance and pluralism, and social, educational, and economic equality) represent the backbone of Israel's civil society. ${ }^{37}$ It is only over the past decade that research efforts have begun to define, for the first time, the very diverse nonprofit sector in Israel (see Gidron \& Katz, 1998), and it continues to be an under-researched field. While keeping in mind the very distinctive political and social circumstances of Israel, it is possible to generally apply the overall contextual framework of American nonprofits to Israeli nonprofits. ${ }^{38}$ Furthermore, as heavily reliant on the contributions from the United States and other foreign countries, Israeli nonprofits operate in a similar funding environment. The following section considers the predominate culture of nonprofits and philanthropic institutions, exploring a variety of factors that have led to an underinvestment in the organizational capacity of nonprofits.

\footnotetext{
${ }^{36}$ This brief discussion of nonprofits in Israel mainly draws attention to nonprofits working towards creating a stronger civil society in Israel. For a much more in depth analysis and attention to the diverse nature and unique history of Israeli nonprofits and their relationship with the state, see Gidron and Katz (1998) or Silber and Rosenhek (1999). See Zeidan and Ghanem (2000) for research on the Palestinian Arab population in Israel.

${ }^{37}$ For a definition of civil society, see Putnam (1993)

${ }^{38}$ Gantz McKay (2005) cites the similarities between the American social change movement and the emerging Israeli social change movement. A main distinction that is made relates to the 'standards' of nonprofit governance. In the U.S., in contrast to Israel, there are groups like the Association of Nonprofits in Maryland and in Minnesota that are setting the standard for expected practices among non-profits in the United States.
} 


\subsubsection{Culture of Nonprofits}

The unique culture of nonprofits around the world is remarkable for its idealism, values and service. Generally coping with complex social issues and taking on significant challenges, nonprofits tend to be a place where a spirit of altruism provides the motivation in the face of minimal resources. This "culture of service," while helping to create mission-driven organizations imbued with a strong sense of commitment, has traditionally led to an underinvestment in organizational capacity (Letts, Ryan \& Grossman, 1999).

Historically, nonprofit leaders have concentrated directly on the delivery of quality services and programs. With the majority of nonprofits founded based on a particular cause, a key priority has been to test and implement an idea that could address a social need (McKinsey \& Co., 2001). Creating social impact through program success has traditionally taken precedence over long term investments in building organizational infrastructures. As part of an effort to ensure that resources were directly devoted to programmatic efforts and often to avoid the impression of wasteful 'overhead' (Sussman, 2004), nonprofits sought to minimize organizational costs. Letts, Ryan and Grossman (1999) point out that, in contrast to the private sector where investment in organizational capacity is considered essential, nonprofits can encounter indifference, if not hostility, to efforts to strengthen their organization capacity. "Programs and organizational capacity are seen as competitors in a zero-sum struggle for limited resources" (p. 32). Considered as a diversion of resources away from the beneficiaries of the service, administrative costs are to be kept to a minimum (Sussman, 2004; McHargue, 2003; Letts, Ryan \& Grossman, 1999).

Generally speaking, the organizational culture of nonprofits, as well as their objective circumstances, provide a series of challenges in terms of investing in overall organizational capacity building. Whether it is the "culture of service" or the simple lack of time that often prevents nonprofits from taking the time to learn, learning and reflection are considered as a luxury that nonprofits cannot afford (Letts, Ryan \& Grossman, 1999). Practices that could potentially take away from the day to day work of providing service are viewed as distracting nonprofits from focusing on fulfilling their mission. Moreover, nonprofits, taking pride in their distinction from the private sector, have traditionally been suspicious of adopting 'business strategies' such as strategic planning or other practices that could prove to enhance performance (Blumenthal, 2003; McKinsey \& Co., 2001). The institutionalization of sound management 
processes and systems is also very difficult in the face of instability and uncertainty regarding sources of funding. Nonprofits can find themselves "often living from hand to mouth", struggling with minimal resources but at the same time trying to spend the majority of time focused on providing their services. (McHargue, 2003, p. 197). The lack of resources also prevents nonprofits from paying commensurate salaries or hiring additional employees to reduce pressures on overburdened staff. Not surprisingly, nonprofits are consequently less able to invest in skill development, reward staff performance or provide monetary incentives to engage in activities that may enhance organizational learning (McHargue, 2003).

\subsubsection{Culture of Philanthropy}

The philanthropic sector, of critical importance to nonprofits in both developed and developing countries, significantly influences the operations of nonprofits as organizations. Unfortunately, the well-intentioned actions of the philanthropic sector have often de-emphasized the importance of organizational capacity; "Donors and funders have traditionally been more interested in supporting an exciting idea than in building an organization that can effectively carry out that idea" (McKinsey \& Co., 2001, p. 19). Historically, foundations have sought to encourage program innovation through grants for short term 'demonstration projects' versus investments in basic organizational needs such as general operating costs (David, 2002, p. 2). ${ }^{39}$

The traditional model of grantmaking, referred to as the "project-funded approach" (David, 2002) or “program-centered approach" (Letts, Ryan \& Grossman, 1999), primarily designates grantee funding for program development and implementation. Often, funders place the greatest emphasis on the creation of new, innovative programs, while leaving questions related to organizational effectiveness and organizational impact unexamined (Firstenberg, 2003). The funding of innovative, pilot programming tends to be, by nature, short term, and does not necessarily foster a nonprofit's ability to build a viable, well-managed institution. ${ }^{40}$ Moreover, it

\footnotetext{
${ }^{39}$ Yet, whereas in the past, new ideas tested by a nonprofit with a foundation's support could lead to government funding, it is less likely to occur today (David, 2002).

40 The emphasis on short term funding for innovative projects creates additional challenges for nonprofits seeking to stabilize their funding sources. Foundations do not like to adopt an idea or program that is already "branded" by support of another foundation (Letts, Ryan \& Grossman, 1999; Weiss et al., 2003). There is the "not invented here" attitude among funders (David, 2002).
} 
is argued that foundations' 'arm's length' oversight role rather than a partnering role with their grantees does not help to ensure that nonprofits focus on improving organizational-wide performance and overall effectiveness (Firstenberg, 2003; Letts, Ryan \& Grossman, 1999). Grantees are not encouraged to assess their overall organizational capacity and are not provided with long-term support that could help them to ensure sustainability. ${ }^{41}$ Table 7 illustrates how grantmaking practices can actually serve to undermine the ability of nonprofits to develop their organizational capacity and long term sustainability (David, 2002; Letts, Ryan \& Grossman, 1999). ${ }^{42}$

Table 7: Traditional Funding Approach to Nonprofits

\begin{tabular}{|l|l|l|}
\hline $\begin{array}{l}\text { Traditional Funding } \\
\text { Approach }\end{array}$ & Rationale of Approach & $\begin{array}{l}\text { Unintended Negative } \\
\text { Consequences }\end{array}$ \\
\hline $\begin{array}{l}\text { Funding programs and } \\
\text { under-investing in } \\
\text { organizational expenses } \\
\text { or administrative costs. }\end{array}$ & $\begin{array}{l}\text { Desire to ensure that the bulk } \\
\text { of resources go directly to } \\
\text { beneficiaries. }\end{array}$ & $\begin{array}{l}\text { Organizations lack resources to } \\
\text { invest in building their capacity. }\end{array}$ \\
\hline $\begin{array}{l}\text { Emphasizing new, } \\
\text { innovative projects. }\end{array}$ & $\begin{array}{l}\text { Fulfilling the unique ability of } \\
\text { the funder to test programs and } \\
\text { design social policy. }\end{array}$ & $\begin{array}{l}\text { Organization can be pushed } \\
\text { beyond their core mission as they } \\
\text { continually "recreate" new } \\
\text { programs to meet funders } \\
\text { interests. }\end{array}$ \\
\hline Investment in short term. & $\begin{array}{l}\text { Ensures that grantees do not } \\
\text { become dependent, while } \\
\text { allowing funders the flexibility } \\
\text { to fund a variety of projects. }\end{array}$ & $\begin{array}{l}\text { Not conducive to long term } \\
\text { planning of organization } \\
\text { capacity. }\end{array}$ \\
\hline Hands off approach. & $\begin{array}{l}\text { Reluctance to "interfere" in the } \\
\text { work of the nonprofit. }\end{array}$ & $\begin{array}{l}\text { Works against the creation of a } \\
\text { partnership where nonprofits can } \\
\text { expose weaknesses and work } \\
\text { with funders to address } \\
\text { organizational challenges. }\end{array}$ \\
\hline
\end{tabular}

\footnotetext{
${ }^{41}$ Letts, Ryan and Grossman (1999) assert that foundations, unlike venture capitalists, do not have "exit strategies" which could help increase the chance of sustainability.

${ }^{42}$ David (2002) poses the question of how do funders improve the "staying power" of their grantees? This question must be asked, as David explains, "We are in the sustainability business, whether we like it or not" (p. 1).
} 
As illustrated in Table 7, the traditional approach of grantmakers has led inadvertently to a lack of investment in nonprofit organizational capacity. For example, funders are reluctant to fund organizational expenses because they want to maximize the amount of resources going directly to beneficiaries. As a result, nonprofit staff may find themselves with sufficient resources to implement a particular project, yet they may have great difficulty finding resources to cover basic organizational expenses such as rent or computer equipment. Under such circumstances, designating funds towards organizational wide-improvement activities can easily feel like an unaffordable luxury. In response to the types of "unintended negative consequences" that are outlined in Table 7 and numerous other challenges facing nonprofits today, efforts to change funding strategies and invest in organizational capacity have gained momentum over the past decade.

\subsubsection{Capacity Building for Nonprofit Organizations}

In recent years, capacity building to enhance organizational performance increasingly has been embraced as a means to help nonprofits better function in an environment that is characterized by both growing needs and shrinking resources. ${ }^{43}$ The nonprofit sector, under great pressure to demonstrate results and enhance accountability (e.g. Light, 2003), has begun to invest in organizational capacity building in an effort to increase effectiveness. Philanthropists and foundations have also been challenged to recognize the changing circumstances and to adopt different grantmaking strategies to improve the organizational performance of their grantees (see David, 2002; Letts, Ryan \& Grossman, 1999; Porter \& Kramer; 1999). While there are certain funders who have always recognized the importance of capacity building, often referred to as 'technical assistance', the overall interest and investment in capacity building has grown significantly in the past decade or so (Light et al, 2004). ${ }^{445}$

\footnotetext{
${ }^{43}$ Based on the adoption of venture capital practices, "venture philanthropy" is gaining increasing attention as a means to promoting nonprofit organizational capacity. Venture philanthropists generally nurture long-term, active partnerships with their grantees, investing a great deal of resources, both financial and other, in helping the grantees to strengthen their organizational capacity (Firstenberg, 2003).

${ }^{44}$ For example, in 2000, American foundations granted \$422 million for management development and technical assistance; in 1994, the amount was $\$ 132$ million (Light et al., 2004).
} 
Touted by nonprofits and funders alike, capacity building has become a 'catch word' in recent years; in fact, McPhee and Bare (2001) argue that it is so "popular and expansive" of a term that there is little agreement on its meaning and " the rhetoric is ahead of the work" (McPhee \& Bare, p. 1).There is a great variety in the types of capacity building programs, with a range of factors shaping each approach such as time frame, focus (i.e., programs designed for individual organizations or for groups of organizations sharing, for example, a geographical or interest community) and type of consulting organization (see Light et al, 2004 or Blumenthal, 2003). ${ }^{46}$ As a result of the exponential growth in the field of capacity building, there is still a great deal of research yet to be carried out regarding its effectiveness and long term impact (Blumenthal, 2003; McKinsey \& Co., 2001). Research efforts represent an attempt over the past few years to provide a conceptualization of capacity building, to evaluate its impact and to greater understand the dimensions of organizational capacity (Light et al, 2004; Blumenthal, 2003; McKinsey \& Co., 2001).

Recent literature in the field of capacity building provides several frameworks that detail the goals of capacity building efforts and the definition of organizational capacity. ${ }^{47}$ Connolly and York's (2003) outline of organizational capacity helps both to provide an overall context and to frame the discussion in the area of adaptive capacity. While keeping in mind the uniqueness of each organizational context and the absence of a 'universal standard' in the long term process of capacity building (Sussman, 2004) four essential organizational capacities can be identified:

1. Adaptive Capacity: the ability of a nonprofit organization to monitor, assess, and respond to internal and external changes.

2. Leadership Capacity: the ability of all organizational leaders to inspire, prioritize, make decisions, provide direction and innovate, all in an effort to achieve the organizational mission.

\footnotetext{
${ }^{46}$ Light et al (2004), for example, separates capacity building programs into three groups: direct response programs, capacity building initiatives and sector-strengthening programs (pp. 24-25). Blumenthal's (2003) typography is divided into capacity grants, development partners and structured programs.

${ }^{47}$ Organizational capacity for nonprofits can be defined in a variety of ways. McKinsey \& Co. (2001), for example, in developing a tool for assessing "capacity" defines capacity in a pyramid of seven elements: 1. Aspirations (mission vision, goals), 2. Strategy (actions and programs), 3. Organizational skills (sum of organization's capabilities), 4. Systems and infrastructure (planning, decision making, knowledge management and administrative systems and physical and technical assets), 5. Human resources (collective capabilities of staff, board, volunteers, etc.), 6.Organizational structure (governance, organizational design, inter-functional coordination, individual job descriptions, etc.), and 7. Culture (shared values, practices, norms and orientation towards performance).
} 
3. Management Capacity: the ability of a nonprofit organization to ensure the effective and efficient use of organizational resources.

4. Technical Capacity: the ability of a nonprofit organization to implement all of the key organizational and programmatic functions (Connolly \& York, 2003, p. 20).

\subsubsection{Adaptive Capacity}

There is often discussion on how nonprofits lack a financial 'bottom line' that exists in the private sector. Yet, in the private sector as Garvin (1999) tells us, "corporate success is best measured by adaptability and flexibility, not the usual short-term measures of profitability and productivity" (p. 9). In both for-profit and nonprofit organizations, organizational learning and ongoing evaluation are significant factors in enhancing overall organizational performance. The ability of any type of organization to continually adapt and to learn from its changing environment is essential for long-term sustainability. For nonprofits, this adaptive capacity is especially vital; David (2002) explains, "to be sustainable in the most fundamental sense (i.e., ongoing demand for its services), a (nonprofit) organization must regularly revisit its mission, strategies and programs to test its continuing efficacy vis-à-vis its intended constituents" (p. 8). While all the core organizational capacities are important for nonprofits, the ability to continually learn from and respond to changes from the external and internal environment is considered the most critical dimension of organizational capacity (Connolly \& York, 2003; Letts, Ryan \& Grossman, 1999). As highlighted previously, organizations face considerable challenges in becoming learning organizations and implementing evaluative practices to enhance learning. Indeed, recent research findings suggest that the weakest component of organizational capacity among nonprofits is their adaptive capacity (Connolly \& York, 2003).

While adaptive capacity is considered to be a fundamental component for enhancing organizational effectiveness, it is a particular aspect of organizational capacity for nonprofits that requires more research (Connolly \& York, 2003). It is important to begin the discussion by reviewing one of the few studies on adaptive capacity that was carried out by Sussman (2004). Sussman identifies four essential characteristics of nonprofit organizations with adaptive capacity: 1) External Focus, 2) Network Connectedness, 3) Inquisitiveness, and 4) 
Innovativeness. ${ }^{48}$ Organizations with an adaptive capacity are "responsive to what is happening outside their organizational boundaries. They consciously interact with their environment which, in turn, provides information-rich feedback, stimulates learning and ultimately prompts improved performance" (p. 3). With an external focus, organizations are "sufficiently porous" to outside perspectives and proactively take steps to ensure that they do not become isolated from their surrounding environment (p. 8). To have a system-wide impact, organizations should be connected to a larger network of organizations. Organizations with this awareness understand that they can more successfully fulfill their mission by developing strategic alliances and interdependent relationships outside of the organization. Inquisitive organizations are "voracious learners," constantly generating and applying new knowledge (p. 16). Acting as "learning organizations," these organizations appreciate the importance of "knowledge management" and "outcome measurement" (p. 16). Lastly, organizations needs to constantly embrace innovation, thereby exhibiting an openness to new ideas, diversity of opinion, and experimentation. Table 8 briefly summarizes the four dimensions of adaptive capacity as defined by Sussman (2004).

Table 8: Four Dimensions of Adaptive Capacity (Sussman, 2004)

\begin{tabular}{|l|l|}
\hline Dimension & Key elements \\
\hline \multirow{1}{*}{ External Focus } & $\circ \begin{array}{l}\text { Awareness of interdependence with surrounding } \\
\text { environment. } \\
\text { Locates resources and capacities from outside of } \\
\text { organization. }\end{array}$ \\
& $\circ \begin{array}{l}\text { Elicits information and ideas from outside the } \\
\text { organization. }\end{array}$ \\
\hline \multirow{3}{*}{ Network Connectedness } & $\circ \begin{array}{l}\text { Construction strategic partnerships. } \\
\text { Board brings in ideas } \\
\text { Affiliations with other organizations and colleagues. }\end{array}$ \\
\hline & $\circ \begin{array}{l}\text { Development of networks to achieve mission } \\
\text { Understanding of potential to create systematic change } \\
\text { through strategic alliances and joint efforts with other } \\
\text { organizations. }\end{array}$ \\
\hline
\end{tabular}

\footnotetext{
${ }^{48}$ Sussman (2004) notes that while the term adapt may suggest "reaction or accommodation to circumstances", the use of adaptive capacity refers to an ability to "generative or initiate change and event to challenge circumstances external to the organization" and not simply react. (p. 4).
} 
Table 8: Four Dimensions of Adaptive Capacity (Sussman, 2004), continued

\begin{tabular}{|c|c|}
\hline 3. Inquisitiveness & $\begin{array}{l}\text { An "appetite for inquiry": seek out data and information } \\
\text { in order to learn, and then apply and share the } \\
\text { knowledge. } \\
\text { Data collection, learning and knowledge development } \\
\text { are an essential, organization -wide effort. } \\
\text { Evaluative activities are considered as a tool for } \\
\text { learning and improving performance. }\end{array}$ \\
\hline 4. Innovation & $\begin{array}{ll} & \text { Creating and implementing new ideas. } \\
\circ & \text { Initiating constant improvement. } \\
\circ & \text { Challenging underlying assumptions and accepted } \\
\text { wisdom. } \\
\circ \text { Rewarding experimentation and risk-taking. } \\
\circ \text { Openness to diversity. }\end{array}$ \\
\hline
\end{tabular}

As illustrated in Table 8, the dimensions of adaptive capacity reflect the concepts associated with a learning organization and the practice of evaluative inquiry. In earlier sections, the challenges involved in applying these principles to all types of organizations was discussed. For nonprofits, research findings suggest that not only is adaptive capacity one of the weakest components of organizational capacity, but that capacity building efforts are not necessarily focusing on strengthening this critical dimension of organizational effectiveness (Connolly \& York, 2003). With a tendency to identify their main organizational capacity needs as related to management or technical issues, nonprofits are not taking a systematic approach to addressing the "underlying problems" or "big picture" issues that can bring about long term improvements in overall capacity (Connolly \& York, p. 29). As reminiscent of earlier discussions on the issue of systems thinking, Fine, Kopf and Thayer (2001) and Blumenthal (2003) also emphasize the vital importance of carrying out capacity building measures that are holistic in their approach to organizational change. Moreover, research has found that the consulting organizations that are providing capacity building assistance are not helping nonprofits to gain a more systematic understanding of how to approach adaptive capacity in particular, and organizational capacity, in general (Connolly \& York, 2003). A specific challenge cited by Sussman (2004 is the 
importance of "capacity balance ${ }^{, 49}$ and the need to find the balance between stability and adaptability. It is suggested that a more holistic approach to nonprofit capacity building and an increased focused on adaptive capacity would take place if consulting organizations invested more effort in assessing the nonprofit's organizational readiness to engage in the capacity building process. A lack of 'organizational readiness' is cited as one of the major reasons for the mismatch that occurs between what type of capacity building efforts are provided and the most pressing needs of the nonprofits (Connolly \& York, 2003).

\subsubsection{Organizational Readiness}

Among the limited number of research studies addressing the effectiveness of capacity building, readiness is considered as one of the main principles of engagement in organizational capacity building (Connolly \& York, 2003; Blumenthal, 2003; Fine, Kopf \& Thayer; 2001). ${ }^{50}$ Fine, Kopf and Thayer (2001) found that the majority of nonprofits, despite their variety (i.e., different stages of organizational life, mission, size and budget), can benefit from some kind of capacity building activities if they demonstrate certain readiness qualities:

1. The organization is open to learning and change.

2. Key organizational members believe that working on organizational issues will contribute positively to the organization's ability to achieve its mission.

3. The organization is able to articulate its mission.

4. The group is prepared to commit the necessary time and resources to the enterprise. (p. 18)

It is valuable to recall from earlier discussions on organizational learning and evaluative practices the influence of culture on change efforts. To survive, an organization needs to be

\footnotetext{
49 In his discussion of capacity alignment and capacity balance, Sussman (2004) refers in general to the categorization of capacity building first developed by Letts, Ryan \& Grossman (1999). This schema divides capacity into three elements: 1. Programmatic Capacity, 2. Organizational Capacity, and 3. Adaptive Capacity.

${ }^{50}$ Fine, Kopf and Thayer (2001) cite eight other principles for effective capacity building: 1 . Adopting a holistic, systems perspective approach 2. Appreciating the organizational context 3. Respecting of the organization's ability to build its own capacity 4. Promoting a culture of ongoing questioning and learning 5. Nurturing team and peer learning 6. Accommodating different styles and incorporating different strategies 7. Ensuring a relationship of trust between the organization and the provider, and 8.Understanding the benefit of a long term approach.
} 
willing to adapt and adjust to its changing environment; yet, at the same time it has a vested interest in maintaining stability and the status quo (Schon, 1983). An organization depends on "the constancies" of organizational life, and "surprise, which is essential to learning, is inimical to smooth organizational functioning" (Schon, p. 327). Any assessment of whether a nonprofit is ready to engage in capacity building efforts, particularly if the goal is to strengthen its adaptive capacity, should first explore the organization's willingness to learn and to change. The 'readiness' of a nonprofit includes the openness of the organization culture. "An organization may seem 'ready' for a specific type of capacity building engagement (as evidenced by strong leadership and adaptability), but the organizational culture may still create challenges to implementation" (Connolly \& York, 2003, p. 41). Too frequently, capacity building efforts begin without a thorough assessment of whether the nonprofit has the commitment to undergo changes or whether the culture is amenable to change.

While further research is needed to understand to a greater extent the factors that make an organization 'ready' to receive all types of capacity building assistance (Connolly \& York, 2003), two studies specifically related to the readiness of nonprofits to engage in evaluative capacity building (Hernandez \& Visher, 2001; Botcheva, White \& Huffman, 2002) can be briefly reviewed. Evaluation is considered a key element of adaptive capacity (see Section 2.4.5). Using evaluation to plan, measure progress, and assess community needs is necessary for ensuring that the organization is relevant to its constituents, effectively meeting its desired outcomes and fulfilling its mission. Hernandez and Visher's study focused on a particular foundation's capacity building initiative to improve grantee performance, an initiative based on the idea that "already successful organizations could be even more effective if they had data that could show what was working and what was not" (Hernandez \& Visher, 2001, p. 5). Their findings underscored that the most critical indicator of success among the participating nonprofits was the level of enthusiasm and motivation among the staff (Hernandez \& Visher, 2001). The 'organizational readiness' and support for evaluation were much more significant than factors such as the organization's size, financial stability, reputation or level of infrastructure. Hernandez and Visher (2001) found that the "mindset mattered as much as tools and techniques"; the establishment of a data collection and measurement system was insufficient alone without an accompanying organizational mindset valuing the process of self-inquiry. 
Another study on evaluative capacity building carried out by Botcheva, White and Huffman (2002), represents an effort on behalf of the researchers to understand the learning culture and outcome measurement practices as indicators of an organization's readiness for research-based evaluation. Twenty-five community agencies completed a survey that included questions about their learning culture and attitudes towards evaluation. The Assessing Learning Culture Scale Items, used to gauge the learning culture of the organization is illustrated in Table 9. ${ }^{51}$ Respondents were asked to rate their organization's learning culture using a 5 point Likert scale (1 "strongly disagree" to 5 "strongly agree").

Table 9: Assessing Learning Culture Scale Items (Botcheva, White \& Huffman, 2002)

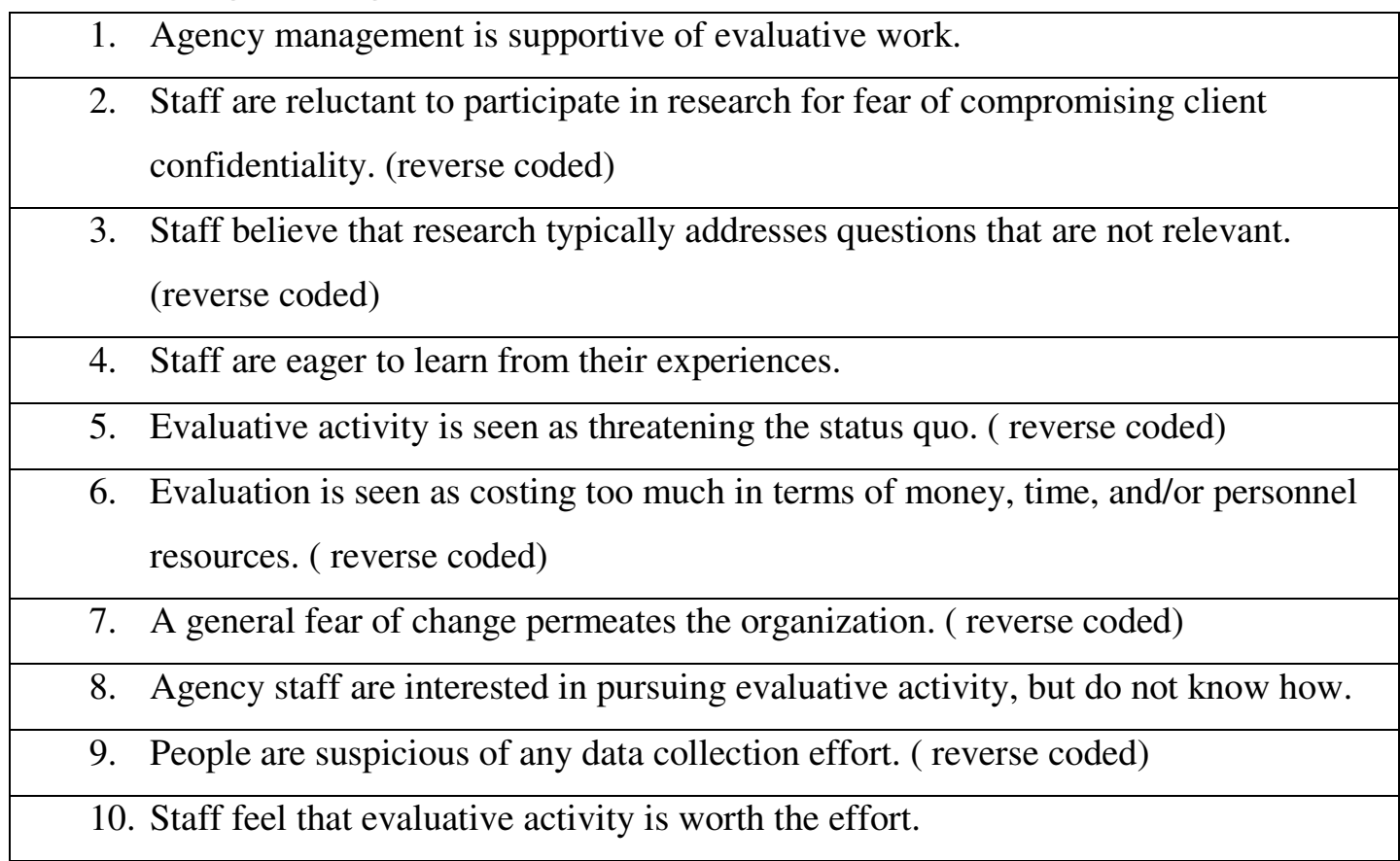

Botcheva, White and Huffman (2002) conclude that the learning culture of an organization is a significant component in the implementation of systematic evaluation. Similar to the conclusions of Hernandez and Visher (2001) and those of Preskill and Torres (1999) (see Section 2.3.2), their findings highlight the importance of factors such as motivation and organizational norms in the success of promoting evaluative capacity.

\footnotetext{
${ }^{51}$ The authors note that the scale was developed based on the work of Preskill \& Torres (1999).
} 
After drawing on various fields of inquiry including organizational learning, evaluative inquiry and nonprofit capacity development, it is possible to begin outlining a conceptual framework for assessing the readiness of an organization to strengthen its adaptive capacity. In this final section of Chapter Two, the implications that the review of the literature hold for the study are considered.

\subsection{IMPLICATIONS FOR THE STUDY}

The sustainability of nonprofits represents one of the most pressing issues facing the public sector today (Wolf, 1999). To remain viable, nonprofits, like all organizations, need to continually adapt themselves to an environment characterized by rapid changes. Certainly for any type of organization, the capacity to learn is considered crucial for ensuring long term organizational stability and productivity (Garvin, 2000; Argyris \& Schon, 1996; Senge, 1990). Evaluation that promotes learning is an essential component; more specifically, participatory models of evaluation can help empower staff, create multiple learning opportunities and promote organizational development (Patton, 1998; Preskill \& Torres, 1999, 2000a; Cousins \& Earl, 1995). Yet, as indicated in the literature, it is often the case that organizations embrace the idea

of learning organizations and evaluation in theory but encounter great challenges in practice (Garvin, 2000; Preskill \& Torres, 1999). In particular regard to nonprofits, organizational capacity building efforts have grown exponentially in recent years. Adaptive capacity, which requires nonprofits to act as learning organizations and to use evaluation as a tool to enhance organizational learning and performance, is considered as the most critical dimension of organizational capacity. Research findings suggest however, that not only is adaptive capacity one of the weakest components of organizational capacity among nonprofits, but that capacity building efforts are not necessarily focusing on strengthening this critical dimension of organizational effectiveness (Connolly \& York, 2003). As first illustrated in Chapter 1, Figure 5, which appears on the following page, outlines some of the challenges that nonprofits face in enhancing their adaptive capacity. 
Figure 5: Review of Barriers to Influencing Social Change

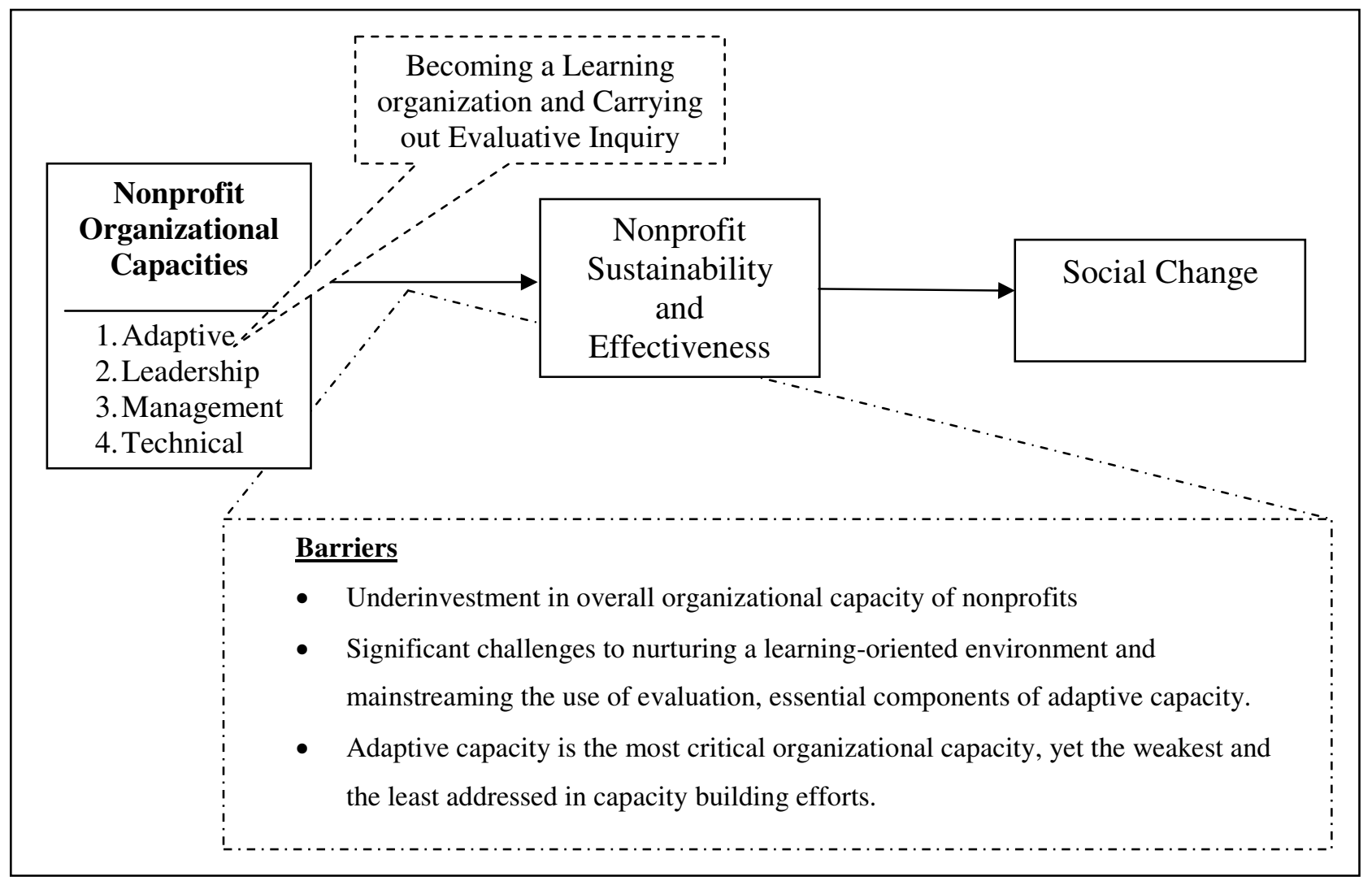

How is it possible to enhance the adaptive capacity of nonprofits? How is it possible to ensure that nonprofits that are receiving help in capacity building sufficiently address the issue of adaptive capacity? It has been emphasized throughout this chapter that the ability to become a learning organization and effectively use evaluation is significantly affected and shaped by an organization's culture that can either inhibit or facilitate learning. Embarking upon organizational change efforts without first understanding the organizational culture will present a considerable barrier to success in the long term. It is necessary to understand the current obstacles to learning or, alternatively, to reinforce the positive aspects of the organization's culture and values. The literature tells us that an essential step towards becoming a learning organization and constructively using evaluation is to assess the current condition of the organization and its readiness for organizational learning (Marsick \& Watkins, 2003; Preskill \& Torres, 2000b; Davidson, 2001). Certainly there is a need for further research in the area of assessment, as the lack of organizational readiness is cited as one of the major reasons for the 
mismatch that occurs between what type of capacity building efforts are provided and the most critical needs of the nonprofits (Connolly \& York, 2003). Strengthening adaptive capacity by nurturing a learning culture and implementing evaluative inquiry are both ongoing, long term processes, and the assessment of the organization's current condition is, of necessity, an important step at all stages of capacity building. Systematic assessment of the organization work environment serves as a guide to change and allows organizations to continually measure their progress.

Drawing from various fields of inquiry including organizational learning, evaluative inquiry and nonprofit capacity development, Table 10 represents a conceptual framework for understanding what makes an organization 'ready' to engage in the ongoing process of adaptive capacity building. The framework is drawn from the two earlier tables, Table 3 and Table 8, which outline the characteristics of a learning organization and of adaptive capacity. The five different dimensions that are delineated below represent a synthesis of these concepts.

Table 10: Understanding Organizational Readiness for Adaptive Capacity Building

\begin{tabular}{|c|c|}
\hline & Description of Concepts \\
\hline Shared Vision & $\begin{array}{l}\text { Creating shared understanding, collectively building a shared purpose. Staff involved in } \\
\text { setting, owning and implementing a joint vision. } \\
\text { Organizational vision integrated with personal vision. Understanding of how job tasks } \\
\text { fulfill organizational goals. } \\
\text { O Articulated learning strategy and investment in long term planning. }\end{array}$ \\
\hline $\begin{array}{l}\text { Inquisitiveness/ } \\
\text { Openness }\end{array}$ & $\begin{array}{l}\text { Embracing 'dissension' and diversity of thought. Willingness to question underlying } \\
\text { assumptions and accepted wisdom. } \\
\text { Rewarding curiosity, risk taking and experimentation. A 'marketplace' for new ideas } \\
\text { with a participatory style of decision making. } \\
\text { Nurturing a safe environment for 'failure'. Learning collectively from past mistakes. } \\
\text { Discussions focus not only on success or noncritical problems. }\end{array}$ \\
\hline $\begin{array}{l}\text { Evaluative } \\
\text { Thinking/ } \\
\text { Systems } \\
\text { Thinking }\end{array}$ & $\begin{array}{l}\text { Understanding interdependence of different parts of organization. } \\
\text { Recognizing patterns of change/ Addressing underlying causes of events/ Acknowledging } \\
\text { the nature of unpredictability. } \\
\text { An "appetite for inquiry": seek out data and information in order to learn, and then apply } \\
\text { and share the knowledge. } \\
\text { Data collection, learning and knowledge development are an essential, organization - } \\
\text { wide effort. Evaluative activities are considered as a tool for learning and improving } \\
\text { performance. }\end{array}$ \\
\hline
\end{tabular}


Table 10: Understanding Organizational Readiness for Adaptive Capacity Building, continued

\begin{tabular}{|l|l|}
\hline Social Capital & $\circ \begin{array}{l}\text { Creating an environment of trust among staff. Ensuring that organizational policies } \\
\text { nurture trust. }\end{array}$ \\
& $\begin{array}{l}\text { Encouraging of group dialogue, communication and collective reflection. Signaling the } \\
\text { importance of knowledge sharing and importance of reciprocity. Rewarding group } \\
\text { success, not just individual. Expectation of staff to work together. }\end{array}$ \\
\hline $\begin{array}{l}\text { Creating opportunities for interaction (providing both time and space). Supporting the } \\
\text { creation of social networks. }\end{array}$ \\
\hline $\begin{array}{l}\text { External Focus/ } \\
\text { Network } \\
\text { Connectedness }\end{array}$ & $\begin{array}{l}\text { Awareness of interdependence with surrounding environment. "Sufficiently porous" to } \\
\text { information and ideas, and locates resources and capacities from outside of organization. } \\
\text { joint efforts with other organizations. / Construction of partnerships or affiliations with } \\
\text { other organizations and colleagues. }\end{array}$ \\
\hline Understanding needs of clients or other organizational stakeholders.
\end{tabular}

In order to gain insight into the adaptive capacity of nonprofits, it is important to assess the current condition of the organization, its work environment and the willingness of its members to engage in learning and change efforts. The detailed framework outlined above serves as the theoretical base for the assessment of adaptive capacity. In the following chapter, the methodology for carrying out the descriptive cross sectional study is explored in depth. 


\subsection{METHODOLOGY AND DATA ANALYSIS}

In this chapter, the methodology used to examine the research questions is outlined. The chapter begins by briefly exploring the context of the study and the guiding research questions and their rationale. This is followed by a description of the site selection, data collection methods, data analysis techniques and the pilot study. The chapter concludes with a discussion of both the strengths and the limitations of the study and an overview of how the research results are presented.

\subsection{CONTEXT OF THE STUDY}

In 1982, an international philanthropic fund, the New Israel Fund (NIF), which is a leading funder of progressive, grass roots nonprofits in Israel, established a capacity building organization to support its grantees. The capacity building organization, entitled Shatil, provides nonprofits with organizational support, training and technical assistance. While there is a rapidly developing national network for nonprofit capacity building in the United States (see Connolly \& York, 2001), Shatil remains a unique type of institution in Israel. Operating in several branches around the country, its target population is social change nonprofits that are working in fields as diverse as civil and human rights, environmental protection, women's issues and social and economic equality.

A particular interest of this study is to focus on social change nonprofits that are creating the base for a civil society in Israel. As the term 'social change' can be interpreted in a variety of ways, it should be noted that the nonprofits under study are seeking to bring about 'social change' by strengthening democracy, equality and human rights in Israel. While recognizing the diversity of nonprofits operating in Israel, this study focuses exclusively on those organizations that can be defined as 'progressive' and 'community-based' or 'grass-roots'. A large number of 
these nonprofits, steadily increasing in number since the 1980's and 1990's, are past and present grantees of NIF and work in some type of cooperation with Shatil. Providing a variety of

services ranging from workshops to individual consultation, Shatil is an important 'address' for these organizations (see Blumenthal, 2004). In an effort to reach social change nonprofits, it was a natural step to contact Shatil and explore the possibilities of designing a study sample. In turn, Shatil was interested in gaining more insight into the process of assessing 'organizational readiness' to engage in capacity building efforts.

\subsection{RESEARCH QUESTIONS AND RATIONALE}

Research findings emphasize the importance of organizational readiness to engage in change efforts; whether the organization is seeking to enhance its learning (Watkins \& Marsick, 1999), use evaluation (Botcheva, White \& Huffman, 2002; Preskill \& Torres, 1999), or strengthen its overall organizational capacity (Blumenthal, 2003; Connolly \& York, 2003). The type of 'readiness' that is the focus of this study is a nonprofit's readiness to engage in adaptive capacity building as reflected in its learning and evaluative culture, and its overall adaptability. The following two research questions seek to address these issues:

\section{$\underline{\text { Research Question \#1 }}$}

How do the nonprofit staff and board members perceive the adaptive capacity of their respective organizations?

What do the nonprofit staff and board members identify as factors that enable them to promote their organization's adaptive capacity, or alternatively, that present ongoing challenges to strengthening their adaptive capacity? The assessment of organizational readiness to engage in change efforts places primary importance on the perspective of the staff regarding their own work environment and learning culture (see Section 2.3.2). Considered an essential step in gauging whether an organization is characterized by a learning environment, staff perceptions shed light on the day to day realities of an organization. Insight can be gained into issues such as 
the level of trust in the organization, the type of communication among staff, the cooperative nature of the workplace, and the openness among leadership to new ideas - all important aspects of adaptive capacity. Recognizing the assessment of organizational stakeholders as a key component in the process towards becoming a learning organization, the study explored the attitudes of both staff and board members towards various dimensions of their respective organization's adaptive capacity.

\section{Research Question \#2}

\section{What are important factors that can be identified as either facilitators or barriers to the ability of these nonprofits to promote their adaptive capacity?}

The study examined factors that may help to explain how these social change organizations are maintaining some form of adaptive capacity. Thus it explored perceptions of how the organizational culture either enhances or inhibits the development of a work environment that is conducive to learning and adaptability. The identification of either facilitators or barriers can ultimately help to shape capacity building efforts; organizations can understand which positive aspects of its learning culture should be reinforced and, alternatively, which challenges to its ability to strengthen adaptive capacity should be addressed.

Investigating the research questions cited above provided insight into how nonprofits are thinking about and are coping with issues related to their learning, adaptability, and ultimately, their sustainability. The research findings with grounded examples can help to inform the discussion among consulting organizations, funders and nonprofits in order to help ensure that capacity building efforts support positive organizational change. 


\subsection{SITE SELECTION}

The ten social change nonprofits organizations participating in the study were drawn from a pool of organizations in contact with a local branch of Shatil. ${ }^{1}$ Organizations were selected based on two criteria. First, the organizations in the sample have been established for at least five years, and they have, at least by one measure, (existence over time), demonstrated some level of adaptive capacity. Second, the first initial step of readiness for any capacity building effort has been taken as a given; the participating nonprofits have determined, at one stage or another, that they could benefit from some type of capacity building assistance, ranging from participation in fundraising workshops to individual consultation, by engaging with Shatil. ${ }^{2}$

Out of the eleven organizations originally identified, ten organizations agreed to participate in the study. The willingness of these organizations to participate in the study also can be considered another indication of their openness to learn as an organization. Effort was taken to select organizations that represent the diversity of the social change movement. Due to the numerically small and intimate nature of the social change nonprofit community in Israel, the confidentiality of the participating organization is maintained. As such, only a very general background of the ten selected nonprofits is outlined in Table 11 on the following page. ${ }^{3}$ The official year of establishment, the mission, activities, scope of engagement (either local, regional or national) and the number of staff are presented.

\footnotetext{
${ }^{1}$ Three of the participating organizations in the sample participated in the pilot study. For this study, there were only minor modifications to the questionnaire and interview process of the pilot study, and the findings of the three pilot study organizations are also included as part of the ten organizations.

${ }^{2}$ There are capacity building programs in the United States where nonprofits are provided with financial incentives to seek out capacity building assistance (see Blumenthal, 2003). The nonprofits working with Shatil do not receive any financial rewards from the New Israel Fund for participation in capacity building efforts.

${ }^{3}$ The research finding are presented across organizations in an effort to ensure confidentiality and to enhance the accessibility of the results. This is discussed further in Section 3.8.
} 
Table 11: Background Information on the Ten Participating Nonprofits

\begin{tabular}{|c|c|c|c|c|}
\hline Founded ${ }^{4}$ & Mission & Activities & $\begin{array}{l}\text { Scope of } \\
\text { Engageme } \\
\text { nt }\end{array}$ & $\begin{array}{l}\text { \# of } \\
\text { Staff }\end{array}$ \\
\hline 1998 & $\begin{array}{l}\text { To promote a civil society by } \\
\text { teaching Russian-speaking } \\
\text { immigrants the principles of } \\
\text { representative democracy and civic } \\
\text { participation. }\end{array}$ & $\begin{array}{l}\text { - Publishing research reports } \\
\text { - Sponsoring courses and } \\
\text { conferences } \\
\text { - Involved in advocacy }\end{array}$ & National & 4 \\
\hline 1999 & $\begin{array}{l}\text { To help low income women, of all } \\
\text { religious and ethnic backgrounds, } \\
\text { to create their own small business }\end{array}$ & $\begin{array}{l}\text { - Teaches business courses } \\
\text { - Provides grants and loans } \\
\text { - Offers ongoing consultation }\end{array}$ & National & 5 \\
\hline 2000 & $\begin{array}{l}\text { To mobilize Arab-Palestinian } \\
\text { young adults (ages 18-30) through } \\
\text { educational programs and cultural } \\
\text { activities. }\end{array}$ & $\begin{array}{l}\text { - Runs youth groups } \\
\text { - Administers a leadership } \\
\text { training course } \\
\text { - Organizes educational and } \\
\text { cultural events } \\
\end{array}$ & National & 4 \\
\hline 1999 & $\begin{array}{l}\text { To help Ethiopian immigrant } \\
\text { families adjust to Israeli life and } \\
\text { integrate children into Israeli } \\
\text { educational system. }\end{array}$ & $\begin{array}{l}\text { - Offers family home visits } \\
\text { - Organizes an after-school } \\
\text { program for Ethiopian } \\
\text { students }\end{array}$ & Local & 6 \\
\hline 1996 & $\begin{array}{l}\text { To discuss the social and human } \\
\text { dilemmas of the Holocaust and to } \\
\text { foster dialogue between Jews and } \\
\text { Arabs based on its universal } \\
\text { lessons. }\end{array}$ & $\begin{array}{l}\text { - Coordinates workshops and } \\
\text { programs }\end{array}$ & Regional & 12 \\
\hline 1996 & $\begin{array}{l}\text { To improve the status of Arab } \\
\text { women by focusing on both } \\
\text { personal and collective } \\
\text { empowerment. }\end{array}$ & $\begin{array}{l}\text { - Offers courses, training, and } \\
\text { mentoring programs } \\
\text { - Provides legal support }\end{array}$ & Regional & 6 \\
\hline 1997 & $\begin{array}{l}\text { To provide a safe haven for } \\
\text { battered women and their children } \\
\text { of all religious and ethnic } \\
\text { backgrounds. }\end{array}$ & $\begin{array}{l}\text { - Provides emergency shelter } \\
\text { - Offers counseling and } \\
\text { services }\end{array}$ & Local & 12 \\
\hline 1999 & $\begin{array}{l}\text { To promote environmental-friendly } \\
\text { policies through advocacy and the } \\
\text { development of community } \\
\text { leadership. }\end{array}$ & $\begin{array}{l}\text { - Organizes community- based } \\
\text { educational events } \\
\text { - Involved in advocacy }\end{array}$ & $\begin{array}{l}\text { Local } \\
\text { branch of } \\
\text { a national } \\
\text { nonprofit }\end{array}$ & 7 \\
\hline $1999^{5}$ & $\begin{array}{l}\text { To meet the social, cultural, legal } \\
\text { and political needs of the gay and } \\
\text { lesbian community. }\end{array}$ & $\begin{array}{l}\text { - Organizes lectures and } \\
\text { organized social activities } \\
\text { - Provides counseling services }\end{array}$ & Local & 1 \\
\hline 1997 & $\begin{array}{l}\text { To promote the social-economic } \\
\text { right of the Arab/Palestinian } \\
\text { community in Israel. }\end{array}$ & $\begin{array}{l}\text { - Involved in advocacy } \\
\text { - Publishes position papers } \\
\text { - Organizes conferences }\end{array}$ & National & 15 \\
\hline
\end{tabular}

\footnotetext{
${ }^{4}$ While this represents the year that the nonprofit was established, many of the nonprofits operated informally for years prior to their official registration with the national government as a nonprofit organization.

${ }^{5}$ The organization was originally established in 1999 as part of a larger community center. In 2004, it became an independent organization run by volunteers and recently received funding for a part-time paid coordinator.
} 


\subsection{METHOD}

Based on the pragmatic approach of utilization-focused evaluation (Patton, 1997), this descriptive cross sectional study employed a mixed-method strategy to gather data. ${ }^{6}$ Prior to carrying out the data collection process, the study received IRB approval (IRB \# 0503217). Both a staff questionnaire and interviews with staff and board members were used to gain insight into the organizations and their work environments. It should be noted that the questionnaire process and the vast majority of the interviews were conducted in Hebrew and consequently translated by the researcher. The researcher, who has a very high level of proficiency in Hebrew, also worked with a native speaker throughout the process of data collection and analysis to ensure that the translations were accurate. The methods employed are discussed below.

\section{Staff Questionnaire}

The staff questionnaire was designed as a data collection tool that would be administered together with in-depth interviews with staff and board members. Used to explore the attitudes of the staff towards their organization's work environment and culture as it relates to adaptive capacity, the questionnaire was considered as the first initial step in the process of assessment (See Appendix A). The qualitative responses, together with the quantitative section of the questionnaire (analyzed with descriptive statistics) thereby provided a preliminary 'picture' of the organization prior to the interview process.

The questionnaire is drawn from the four assessment tools (Watkins \& Marsick, 1999; Preskill \& Torres, 2000b; Davidson, 2001 and Botcheva, White \& Huffman, 2002) that were reviewed in detail in Chapter Two. ${ }^{7}$ Three of the assessment tools were developed for use in forprofit organizations and the researcher therefore modified many of the items in order to incorporate appropriate language for a nonprofit context. Several questions were also developed

\footnotetext{
${ }^{6}$ This study embraces the pragmatic approach towards the use of qualitative and qualitative methods while recognizing that there are those who argue that that both the political nature of evaluation as well as its philosophical underpinnings must be taken into account in determining methodology. For example, see Greene (1998), who states, " it is because evaluation is politically contextualized that constitutive differences in evaluation methodologies extend well beyond alternative methods and also beyond alternative philosophies of science (see Guba, 1990) to incorporate alternative ideologies (Scriven, 1983) and alternative philosophies of ethics, democracy and justice (House, 1980)" (p. 375).

${ }^{7}$ For the origin of each item on the questionnaire see Appendix B.
} 
by the researcher to ensure that the questionnaire touched on various aspects of adaptive capacity that were not addressed in the existing instruments. In addition, open-ended items were added to provide the staff with an opportunity to articulate perspectives on issues in their own words. Divided into the following four sections, the staff questionnaire includes a total of thirty-two close-ended items and eight open-ended items:

1) Organizational Planning - 5 close-ended items (based on a Likert Scale ranging from 1-5, 1 being strongly disagree to 5 strongly agree) and 2 open-ended items (Question \#s 1-7 on the questionnaire).

2) Organizational Work Environment - 11 close-ended items (based on a Likert Scale ranging from 1-5, 1 being strongly disagree to 5 strongly agree), 9 close-ended items (based on a Likert Scale ranging from 1-5, 1 being almost never to 5 almost always), and 4 open-ended items (Question \#s 8-31 on the questionnaire).

3) Organizational Assessment of Progress - 4 close-ended items (based on a Likert Scale ranging from 1-5, 1 being strongly disagree to 5 strongly agree) and 1 open-ended item (Question \#s 32-36 on the questionnaire).

4) Relationship with Other Organizations -3 close-ended items (based on a Likert Scale ranging from 1-5, 1 being strongly disagree to 5 strongly agree) and 1 open-ended item (Question \#s 37-40 on the questionnaire).

In order to provide a logical ordering that could be understood by staff members, the questions were purposely grouped together by the four topics listed above. In the subsequent analysis of the questionnaire findings, the items were categorized according to the five key dimensions of adaptive capacity (1. Shared Vision, 2. Inquisitiveness, 3. Evaluative Thinking/Systems Thinking, 4. Social Capital, 5. Network/ External Connectedness). The issues raised in the questionnaire gave staff members the opportunity to be introduced to these issues and to consider how they apply to their respective organization. 
The questionnaires were administered to the staff by the researcher. Whenever possible ( 8 out of 10 cases), the researcher attended a staff meeting in order to explain the goals of the study and distribute the questionnaire at the end of the meeting. ${ }^{8}$ Staff members then filled out the questionnaire at their work spaces and returned it directly to the researcher. ${ }^{9}$ The staff questionnaire took the participants approximately fifteen minutes to complete. In terms of the response rate, $100 \%$ of the staff filled out the questionnaire in six of the organizations. For the four additional organizations, the response rate was $67 \%, 75 \%, 80 \%$ and $92 \%$. In total, sixty- six questionnaires were filled out (the data collected in summarized in Table 12). The questionnaire was especially important to ensure that the views of the all the staff members were represented, and to provide an initial picture of the organization prior to the interviews. In the four organizations without a $100 \%$ response rate, special effort was taken to interview staff members who were absent when the questionnaires were distributed.

\section{$\underline{\text { Staff and Board Interview Protocol }}$}

To examine in depth various aspects of adaptive capacity, interviews among the staff and board of the participating nonprofits were carried out. The interview protocol addressed a variety of issues that were raised in the questionnaire, including questions relating to the work environment such as the level of cooperation among the staff, the manner in which conflict is handled and the degree to which feedback is encouraged by the organizational leadership. A sample of additional questions is provided below (See Appendix C for the Interview Protocol).

- What are major changes that have occurred in this organization in the last five years?

- What changes do you see coming in the next few years and how do you know they are coming?

- How do you ensure that you are meeting the needs of your community base?

- How would you distinguish your organization's 'character' from other organizations? What makes your organization "special"?

While the protocol provides an overall framework for the discussion, there was flexibility in the sequence and wording of the questions (Patton, 2002). During the qualitative interviews, which

\footnotetext{
${ }_{9}^{8}$ In two organizations, the researcher distributed the questionnaire during regular work hours.

${ }^{9}$ In two organizations, the researcher distributed the questionnaire during staff meetings and the questionnaires were mailed directly back to the researcher. In three organizations, staff members that were not present at the staff meeting where questionnaires were collected mailed them directly back to the researcher.
} 
usually lasted between forty-five minutes to an hour, the researcher was able to probe deeper into the topics that are addressed in the questionnaire.

In smaller organizations (four or less staff members), the entire staff was interviewed. In larger organizations, the executive director and at least three professional staff members were interviewed. The researcher added additional interviews (up to three staff members) when it was deemed necessary in order to try to receive as comprehensive a picture of the organization as possible. Interviews were added when there was not a $100 \%$ response rate on the questionnaires or when the data from the first set of interviews was not reflective of the questionnaire results. Additional staff members were thus interviewed in an effort to ensure that a variety of perspectives was represented in the data. In general, the staff members were chosen according to their availability based on the designated day that the researcher conducted interviews at the selected nonprofit. Effort was taken to interview a variety of staff members in terms of their position and role in the organization. When additional staff interviews were carried out, (either because staff members of organizations under five members were not present or the researcher determined that it was important to interview additional members of the organization), the researcher directly contacted the staff members to coordinate meetings. In addition, one board member was interviewed from each of the ten nonprofits. The board members were chosen as a sample of convenience. The researcher selected the interviewee from a complete list of the board members provided by the organization, and carried out interviews with those who were readily available.

In total, forty-three interviews were carried out face to face with staff members of the ten nonprofits. The interviews generally took place at the location of the nonprofit during regular work hours. The interviews were held in a quiet space that was separated from the work flow of the office and allowed for privacy from other staff members. If privacy was not possible at the workplace, interviews were carried out at a nearby informal setting such as a local coffee shop. With regard to the board members, the manner and location of the interview was arranged at the convenience of the interviewee. Out of the ten board member interviews, seven were carried out over the telephone and three were carried out at local meeting place. 
Summary Table of Study Sample and Data Collected

Table 12 summarizes the data collected from each of the ten participating organizations. For each organization, three 'numbers' ('N') are presented: the number of staff members, the number of staff members who filled out a questionnaire (followed by the response rate in the form of a percentage), and the combined number of staff and board members who were interviewed. ${ }^{10}$

Table 12: Data Collected from the Participating Organizations

\begin{tabular}{|c|c|c|c|}
\hline Organization & $\begin{array}{l}\text { Number of } \\
\text { Staff }\end{array}$ & $\begin{array}{l}\text { Number of } \\
\text { Questionnaires }\end{array}$ & $\begin{array}{l}\text { Number of } \\
\text { Interviews }\end{array}$ \\
\hline $\mathrm{A}$ & $\begin{array}{l}7 \text { staff } \\
\text { members }\end{array}$ & $\mathrm{N}=7(100 \%)$ & $\begin{array}{l}1 \text { Director } \\
3 \text { Staff Members } \\
1 \text { Board Member } \\
\mathrm{N}=5\end{array}$ \\
\hline B & $\begin{array}{l}12 \text { staff } \\
\text { members }\end{array}$ & $\mathrm{N}=8(67 \%)$ & $\begin{array}{l}\text { 1 Director } \\
4 \text { Staff Members } \\
1 \text { Board Member } \\
\mathrm{N}=6\end{array}$ \\
\hline $\mathrm{C}$ & $\begin{array}{l}4 \text { staff } \\
\text { members }\end{array}$ & $\mathrm{N}=4(100 \%)$ & $\begin{array}{l}1 \text { Director } \\
3 \text { Staff Members } \\
1 \text { Board Member } \\
\mathrm{N}=5\end{array}$ \\
\hline $\mathrm{D}$ & $\begin{array}{l}5 \text { staff } \\
\text { members }\end{array}$ & $\mathrm{N}=4(80 \%)$ & $\begin{array}{l}1 \text { Director } \\
3 \text { Staff Members } \\
1 \text { Board Member } \\
\mathrm{N}=5\end{array}$ \\
\hline $\mathrm{E}$ & $\begin{array}{l}4 \text { staff } \\
\text { members }\end{array}$ & $\mathrm{N}=4(100 \%)$ & $\begin{array}{l}\text { 1 Director } \\
3 \text { Staff Members } \\
1 \text { Board Member } \\
\mathrm{N}=5\end{array}$ \\
\hline $\mathrm{F}$ & $\begin{array}{l}15 \text { staff } \\
\text { members }\end{array}$ & $\mathrm{N}=15(100 \%)$ & $\begin{array}{l}1 \text { Director } \\
6 \text { Staff Members } \\
1 \text { Board Member } \\
\mathrm{N}=8\end{array}$ \\
\hline
\end{tabular}

${ }^{10}$ To further protect their confidentiality, the ordering of the organizations in Table 12 differs from the order of organizations presented in Table 11. 
Table 12: Data Collected from the Participating Organizations, continued

\begin{tabular}{|c|c|c|c|}
\hline Organization & $\begin{array}{l}\text { Number of } \\
\text { Staff }\end{array}$ & $\begin{array}{l}\text { Number of } \\
\text { Questionnaires }\end{array}$ & $\begin{array}{l}\text { Number of } \\
\text { Interviews }\end{array}$ \\
\hline $\mathrm{G}$ & $\begin{array}{l}5 \text { staff } \\
\text { members }\end{array}$ & $\mathrm{N}=5(100 \%)$ & $\begin{array}{l}\text { 1 Director } \\
4 \text { Staff Members } \\
1 \text { Board Member } \\
\mathrm{N}=6\end{array}$ \\
\hline $\mathrm{H}$ & $\begin{array}{l}12 \text { staff } \\
\text { members }\end{array}$ & $\mathrm{N}=11(92 \%)$ & $\begin{array}{l}1 \text { Director } \\
3 \text { Staff Members } \\
1 \text { Board Member } \\
\mathrm{N}=5\end{array}$ \\
\hline I & $\begin{array}{l}5 \text { staff } \\
\text { members }\end{array}$ & $\mathrm{N}=5(100 \%)$ & $\begin{array}{l}\text { 4 Staff Members }{ }^{11} \\
1 \text { Board Member } \\
\mathrm{N}=5\end{array}$ \\
\hline $\mathrm{J}$ & $\begin{array}{l}1 \text { staff } \\
\text { member } \\
3 \\
\text { committee } \\
\text { members }\end{array}$ & $\mathrm{N}=3(75 \%)$ & $\begin{array}{l}2 \text { Committee } \\
\text { Members } \\
1 \text { Staff Member } \\
\mathrm{N}=3\end{array}$ \\
\hline & & $\begin{array}{l}\text { Total \# of } \\
\text { Questionnaires: } 66\end{array}$ & $\begin{array}{l}\text { Total \# of Interviews: } \\
53 \\
\text { (45 face to face) } \\
\text { (7 by phone) }\end{array}$ \\
\hline
\end{tabular}

\subsection{DATA ANALYSIS TECHNIQUES}

In this descriptive cross sectional study, data from both the questionnaire and the interviews were analyzed according to the theoretical framework for adaptive capacity outlined below (see Section 2.5 for a detailed description of the framework). The five key attributes of adaptive capacity outlined on the following page are as follows:

\footnotetext{
${ }^{11}$ Both Organizations I and J have flat hierarchical structures and do not have a director.
} 
1. Shared Vision

2. Inquisitiveness/ Openness

- Future Scanning

- Open to New Ideas/ Diversity of thought

- Open to Change

- Challenge assumptions

- Take risks, discuss mistakes

3. Systems Thinking/ Evaluative Thinking

- Systems and encouragement for sharing knowledge

- Interrelatedness

- Evaluative Attitude

4. Social Capital

- Inquiry

- Dialogue

- Critical feedback

- Cooperation

\section{External Focus/ Network Connectedness}

Based on the coding of each question according to theoretical dimensions of adaptive capacity building, the quantitative results of the questionnaire were analyzed. Each question was categorized by the components of adaptive capacity (See Appendix D). Descriptive statistics were calculated for the thirty-two close-ended items (e.g. mean, frequency and standard deviation). The purpose of the statistical analysis of the questionnaire was to provide a general picture of the organizational work environments prior to carrying out the interviews. As such, only the organizational mean is reported in the findings.

The qualitative data from the questionnaire and from the interviews were organized into an analytical framework by issues (Patton, 2002); the results were coded along the dimensions of adaptive capacity and compiled together with the questionnaire results (See Appendix E). By analyzing the results according to the dimensions of adaptive capacity, the study organized the data in order to highlight key issues (Patton, 2002) related to the overall work environment and learning culture. To help strengthen the reliability of the interview data, the researcher summarized the main findings of the discussion upon the conclusion of the interviews for the interviewee. In addition, a colleague of the researcher coded an entire set of interviews from one of the participating organizations. The codings of the researcher and the colleague were 
compared in a further effort to ensure reliability in the coding of interviews. ${ }^{12}$ In general, the interpretations were parallel; using the same data base, similar themes were generated by both sets of codings.

\subsection{PILOT TEST}

A pilot study was conducted with three of the ten nonprofit organizations in the sample. Prior to its implementation, the pilot study received IRB approval (IRB \# 0411023). All the staff members of the three organizations completed the questionnaire (organizations of seven, fifteen and five staff members) (see Appendix F for the pilot questionnaire). Interviews were carried out with four staff members (out of seven), five staff (out of five), seven staff (out of fifteen) and one board member from each organization. The pilot study provided an opportunity for the researcher to receive feedback on the questionnaire and interview process, as well as to test the data collection and analysis plan.

Upon the completion of the interviews in the pilot study, staff members were asked to consider the clarity and relevance of the questions for both the interviews and the questionnaire. With respect to the questionnaire, the Hebrew language version of the questionnaire was considered to be clearly articulated (footnotes defining words such as stakeholder and evaluation had been added to ensure that the translated words were clearly understood). Feedback was received, however, regarding a particular aspect of the questionnaire's design. Several participants reported that the Likert scale of 1-5, strongly disagree to strongly agree did not allow them to provide what they felt was an appropriate response. It was determined that a more fitting response to a specific group of questions would be a Likert scale that is time based versus judgment based. For example, rather than answering the question "my staff is open to feedback" on a Likert scale ranging from strongly agree to strongly disagree, the choice would be modified to a Likert scale ranging almost never to almost always. ${ }^{13}$ By transforming the answer choices to be based in frequency of action, responses could be more grounded in behavior within an organization versus an attitudinal stance.

\footnotetext{
12 For samples of coded interviews, see Appendix E

13 While recognizing that the change of scales from level of agreement to frequency would provide more clarity, the results can be similarly interpreted. Therefore the findings from the pilot study on questions are factored in to the analysis of the findings. Only those questions that did not appear on both the pilot questionnaire and on the study questionnaire will not be reviewed in Chapter Four. For more details on the reporting of the results, see Section 3.8 .
} 
During the analysis of the pilot test results, it became apparent that the study could be enhanced by modifying the questionnaire. For example, the researcher came to the conclusion that one of the questions, drawn from Botcheva, White and Huffman; 2002, was too ambiguous; there were varying interpretations of a response to the question "In my organization, staff are interested in pursuing evaluative activity but do not know how". In addition to removing some items, several questions directly focusing on organizational leadership (regarding issues of feedback and decision-making) were added to the questionnaire. Lastly, five open-ended questions, relating to the concepts raised in the closed ended items, were considered as an important addition to the questionnaire. In the reporting of the results, there is only specific reference to those questions that appeared on both the pilot survey and the study survey. The limitations and strengths of the study are addressed in the following section.

\subsection{LIMITATIONS AND STRENGTHS OF THE STUDY}

The design of this descriptive cross sectional study is based on the premise that adaptive capacity can be assessed in meaningful ways by evaluating the attitudes and perceptions of the nonprofit staff. Assessment processes, considered an essential part of the ongoing process towards becoming a learning organization, place primary importance on the perspective of the staff towards their own work environment (see Section 2.3.2). This study did not connect a 'score' of adaptive capacity to the organization's overall performance or identify a single definition of 'merit' as is the stated goal in the Davidson (2002) study. Empirical data about the extent to which any perceptions of adaptive capacity actually influence the performance of the nonprofit are therefore not provided. Rather, the study examined the perspectives of the staff and board of the nonprofits in order to gain insight into how well the organizations in the sample have developed their adaptability and their capacity for learning.

This study was conducted among a small research sample of social change nonprofits that are, in a certain sense, self-selected. The target population for the study was nonprofits that had already expressed a willingness to be involved in capacity building efforts. By design, the nonprofits under study had already made the decision to enhance their organizational capacities, and as such, the 
sample of nonprofits may exhibit higher levels of adaptive capacity. One of the main objectives of the study was to help focus the discussion between consulting organizations and nonprofits so that capacity building efforts are effective in supporting positive organizational change. Therefore, it is possible that the nonprofits with the greatest need for strengthening adaptive capacity are not under study.

Various limitations to the data collection and analysis process should be noted. First, there is always the possibility that items from the questionnaire were interpreted differently by staff members, or that respondents underreported existing problems in the work environment. The mixed method approach to data collection helped to mitigate this potential limitation through the use of in-depth qualitative interviews. In regards to data analysis, the boundaries of the framework for adaptive capacity building are somewhat artificial. The five major attributes of adaptive capacity interrelate, overlap, and affect one another. While this relationship presents some difficulty in carrying out the analysis of the questionnaire and providing results on specific dimensions of adaptive capacity, this framework provides a highly accessible manner for the readers to understand the research findings. The issues addressed above are further discussed in Chapter Five. The final section of this chapter provides a brief description of the reporting of the research findings.

\subsection{PRESENTATION OF THE RESEARCH FINDINGS}

The research findings from this descriptive, cross sectional study are presented in the following chapter according to the five key dimensions of adaptive capacity. In the reporting of the results, the data from the research will be integrated into the literature on nonprofits and organizational learning. Each dimension of adaptive capacity is discussed in detail by section with a general discussion of the findings across organizations. Both due to the similarities found between the participating organizations and to maintain the confidentiality of the nonprofits, the findings are presented across organizations. The discussion focuses on the overall themes that emerge from the ten nonprofits in the sample, with references to particular organizations in certain circumstances. Accordingly, thematic perspectives of the organizations will be explored. 
Each section begins with a brief introduction of the particular aspect of adaptive capacity, followed by a general characterization of the findings related to the specific attribute. While reporting the results of the study, each section will integrate the research findings into the existing literature on the topic. The first research question of the study is addressed during the discussion of staff and board perceptions of their work environment and learning culture. The second research question is then explored during a discussion of major facilitators and barriers that were identified based on an analysis of the data. A key facilitator and barrier for each aspect of adaptive capacity was selected according to both its degree of significance and its prevalence among the nonprofits in the sample. It is not intended to be a comprehensive list of all the facilitators and barriers that impact on the effectiveness and performance of nonprofits. Current efforts of capacity building that the nonprofits in the sample are undergoing are also be integrated into the discussion.

In reporting the questionnaire results, the organizational mean of all ten participating organizations (which includes the three organizations that participated in the pilot study) for the close-ended items are reported. The questionnaire results by organization are presented in Appendices $\mathrm{G}$ and $\mathrm{H}$ (the organizations are listed in random order in a further effort to protect their confidentiality). If there was wide divergence of an organization from the organizational mean, it is noted. As previously mentioned, only those items on the questionnaire that appeared on both the pilot survey and the study survey are addressed in the results section.

In specific regard to the open-ended items of the questionnaire, the responses of participants are used to illustrate issues raised by the staff. While the open-ended items hold value in highlighting key sentiments of the staff, their value for statistical analysis is limited. There are several reasons why it is not possible to draw conclusions from the quantitative number of the open-ended items: 1) Three of the organizations did not answer open-ended questions; 2) Numerous respondents skipped various open-ended items; and 3) The number of staff per organizations varied and therefore skewed the results. The number of respondents choosing to answer particular items on the open-ended sections (i.e., the number of those who chose to complete the item: "We have a cohesive organizational strategy because..." versus the number of those who chose to complete the item "We would have a more cohesive organizational strategy for the future if...”) are noted in the footnotes only. 


\subsection{RESULTS}

In this chapter, the research findings are presented according to the five key dimensions of adaptive capacity (see Section 2.5). In each of the five sections, the general characteristics of the ten organizations as related to each dimension of adaptive capacity are addressed, followed by a discussion of a key facilitator and a key barrier to enhancing adaptive capacity. Relevant literature will be integrated throughout the discussion. The two research questions, as outlined below, are examined within each section on a particular aspect of adaptive capacity.

\section{$\underline{\text { Research Question \#1 }}$}

How do the nonprofit staff and board members perceive the adaptive capacity of their respective organizations?

\section{Research Question \#2}

What are the important factors that can be identified as either facilitators or barriers to the ability of these nonprofits ability to promote their adaptive capacity?

In addressing the research questions cited above, the findings focus on the overall themes that emerged from the ten nonprofits, and references to particular organizations are only noted in a few circumstances. The rationale for presenting the results in this manner is twofold. First, the nonprofits share much in common in terms of what affects their readiness to strengthen adaptive capacity (if there are particular anomalies, they are noted in the findings). The second rationale for this approach is due to the small, intimate nature of the social change movement in Israel and the possibility of easily identifying participating nonprofits even without names. In an effort to protect their confidentiality, the particular experiences of the nonprofits are conveyed only when the information does not compromise them. ${ }^{14}$

In reviewing the results of the research, it is important to consider once again the unique context of the nonprofits under study. The nonprofits in the sample and other like-minded

\footnotetext{
${ }^{14}$ For more details on how the research results are presented, see Section 3.9.
} 
organizations are at the forefront of the growing social change movement in Israel. Many of these nonprofits are promoting the values of a democratic and civil society, an especially challenging goal in light of the violence and uncertainty that has characterized the Israeli political landscape. Sustaining oneself in this environment, as all of the nonprofits in the sample have done for at least five years, indicates a fundamental level of adaptive capacity. At the same time, these organizations are coping with the typical challenges facing nonprofits, such as a lack of funding or under-investment in organizational infrastructure. While keeping this context in mind, it is informative to explore their experiences and perspective regarding adaptive capacity.

The results are presented in the following discussion according to each of the five dimensions of adaptive capacity. ${ }^{15}$ As such, the five sections are organized as follows:

1. Shared Vision

2. Inquisitiveness/ Openness

3. Systems Thinking/ Evaluative Thinking

4. Social Capital

5. External Focus/ Network Connectedness

\subsection{SHARED VISION}

Why is a shared vision so essential to adaptive capacity? In a learning organization, according to Senge (1990), everyone needs to be able to easily answer the question "What do we want to create?"(p. 206). In organizations that have a shared understanding of how to answer this question, the vision can act as an important 'anchor' that provides stability in the midst of organizational adaptation and change. Each member of the organization should not only easily be able to answer the question: "What do we want to create?" but also be able to answer the question: "How do we want to create it?" 16 Shared vision includes both an understanding of the overall mission and goals of the organization, as well as a shared understanding of the specific actions

\footnotetext{
${ }^{15}$ It is important to note that the distinctions between the five aspects are adaptive capacity are important for the sake of discussion, however they are interrelated and overlapping in nature. For example, if the organization has an open environment characterized by cooperation, it is more likely to have a higher level of social capital (See Subsection 2.1.2).

${ }^{16}$ See Subsection 2.1.2.1
} 
that the organization will take in order to achieve its mission. ${ }^{17}$ Overall, the staff members of the nonprofits in the sample demonstrate a strong commitment to and a clear understanding of their organization's mission. However, at the same time, these nonprofits sometimes encounter difficulties in developing a cohesive strategy and long-term plan for the future.

In general, staff members exhibit a dedication to the mission of their respective organizations. An open-ended item in the questionnaire asked respondents to complete the sentence, "The goal of the organization is to...". There was a strong correlation on the open-ended responses among staff members, thereby indicating a high degree of shared understanding among the staff regarding the vision of their respective nonprofit. Respondents were then asked to mark, on a scale of 1-5, (with 1: strongly disagree and 5: strongly agree), the extent to which they agree with the following statements: "I really feel like my work makes a difference in people's lives" and "In my organization, staff understand how their work relates to the goals or mission of the organization". For the item "I really feel like my work makes a difference in people's lives" the mean score for the ten organizations is 4.3. The organizational mean is 4.2 for the item "In my organization, staff understand how their work relates to the goals or mission of the organization" (For results by organization, see Appendices G and H).

As part of a relatively new social change movement in Israel, the nonprofits in the sample have all been established within the past ten years, and a large number of the current staff are part of the original founders. Many of the staff members worked for years as volunteers prior to the official establishment of the nonprofit and have demonstrated great personal commitment to the vision of the organization. During the interviews, staff members often spoke nostalgically of the early beginnings of the organization and discussed how involved they have felt in both shaping and implementing its vision. A large number of the nonprofits, especially those working with minority or immigrant communities, undoubtedly can be considered as 'pioneers' in the Israeli social change movement, and many of these nonprofits remain unique in their mission. What makes these nonprofits innovative is not just their mission and goals; it is also the very concept of an unaffiliated nonprofit organization working in the field of social change. Not surprisingly, staff members generally exhibit a strong sense of pride in their organizations. As indicated in the interviews, this dedication to the organizational vision among the staff is also accompanied by a

\footnotetext{
${ }^{17}$ For a more detailed discussion on the distinction between organizational vision and organizational strategies, see McKinsey \& Co., (2001).
} 
keen sense of immediacy and urgency about their work. Operating in the highly volatile Israeli social-political context, the staff of these nonprofits have to remain especially motivated and committed to their organizational vision.

The visions or aspirations, an articulation of a nonprofit's "common sense of purpose and direction", should be distinguished from strategy, which is "the coherent set of actions and programs aimed at fulfilling the organization's overarching goals” (McKinsey \& Co., p. 33). While there may be general agreement surrounding the organizational mission, staff members in the sample indicate that views on how to implement the vision do not necessarily enjoy as much of a consensus. For example, staff members were asked to rate on a scale of 1-5 ( with 1 strongly disagree and 5 as strongly agree) the reverse coded question "The staff I work with have very different opinions from each other about where our organization needs to improve". After recoding the responses, the organizational mean was only 3.1. One can gain more insight into this issue by reviewing the responses in the open-ended section of the questionnaire. Respondents were asked to complete one of the following two sentences: "We have a cohesive organizational strategy because... or We would have a more cohesive organizational strategy for the future if..." Those respondents who chose to complete the sentence, "We have a cohesive organizational strategy because" discussed a variety of issues related to shared understandings of the organizational goals. ${ }^{18}$ For example,

- "Our rationale is clear, it is checked every few years, and we proceed cautiously towards our goals." 19

- "We are together ideologically, and socially, we are all here because of our values and beliefs."

- "We know where we are aspiring to, and what are the directions that we want to go."

18 Out of the 31 responses, 15 respondents completed the sentence: We would have a more cohesive organizational strategy for the future if ... and 16 respondents chose to complete the sentence: We have a cohesive organizational strategy because... Please Note: While the open-ended items hold value in highlighting key sentiments of the staff, their value for statistical analysis is limited and will therefore only appear in footnotes throughout Chapter Four. There are several reasons that it is not possible to draw conclusions from the quantitative number of the open-ended items: 1.) Three of the organizations did not answer open-ended questions 2.) Numerous respondents skipped various open-ended items, and 3.) The number of staff per organizations varied and therefore can skew the results (See Section 3.9 for the Presentation of Research Findings).

19 The quotations that appear throughout Chapter 4 are translated from Hebrew by the researcher. 
Yet for those respondents who chose to complete the sentence "We would have a more cohesive organizational strategy for the future if ...", a variety of difficulties were cited:

- " "If our staff would understand that the goals of the organization have to be crystallized."

- "If we could promise budgetary stability or even more, a budgetary plan of the organization. It is impossible to plan the strategy for programs without being sure of the continued existence of our projects."

- "If we would arrive at a better agreement between us on our options to create change and if we would learn more about the practical options that are open to us."

- "If we would define in a more detailed way our target population and the place we see the organization, for example, in the next five years."

- "If there was more facilitation focused on this issue and we were not allowed to avoid discussing it."

Respondents also mentioned the need to simply invest more effort in the process, raising problems such as time restrictions or the inability to focus on long-term strategies.

It is uncertain as to what degree the nonprofits in the sample are successfully considering and mapping a long-term strategy. For example, respondents were asked to indicate how much they agree with the following statement: "We are just trying to survive day to day and do not have the time to consider long term plans for our organization". The question is reverse coded, and with a scale of 1-5 (with 1 strongly disagree and 5 as strongly agree), the organizational mean after recoding was 4.1. On another item with a similar scale based on the level of agreement: "We are actively planning in anticipation of how the external environment (our client base, policy changes, etc.) may change over the next few years," the organizational mean was 3.8.

Strategy should "build on a nonprofit's core competencies, allocate resources to priorities and help delineate its unique point of differentiation" (McKinsey \& Co., 2001, p. 41). Rather than simply a reflection of the organization's activities, strategies should be aligned with an organization's vision and the organization's capacities. (McKinsey \& Co., 2001). Interviews with staff members help provide insight into the issue of long-term strategic planning among the nonprofits. According to staff members, several of the nonprofits are encountering problems in articulating a strategy; they are having difficulties reaching consensus or providing sufficient clarity regarding the specific goals that are designed to achieve the mission. For example, the director of a nonprofit dealing with advocacy voiced his concern over how successfully they are juggling a variety of competing priorities: 
We need to match our strategies for each goal. I feel that the staff is committed and believe in the work, but we are not always checking ourselves and seeing if the investment of our time is worth it. It is very difficult for us to connect the goals of the organization with the investment of time and to understand that if we do A, it will lead to B which will lead to $\mathrm{C}$.

A staff member of the same organization expressed frustration with the lack of strategic planning:

We need to spend more time planning, and not just advance by inertia. If we are not pushed by a dilemma, then we do not ask if our strategy is right or wrong. Otherwise we do not focus on the strategy or ask the hard questions. We never have time for it... We have too many missions. It is like we are standing there holding our finger on a hole in the dam.

Even when nonprofits do have a well-conceived strategy, there is always the challenge of 'staying on mission'. A particular difficulty that was articulated by staff members in the interviews is the ongoing dilemma of remaining true to the vision of the organization. For example, a nonprofit that promotes civil society is regularly 'tempted' to establish other types of educational activities for immigrant communities. The director explained,

It is always easier to receive funding for more 'mainstream' educational activities such as teaching Hebrew or job training skills. So far we have survived the different periods of our establishment and have not lost our vision and we have not given in to the temptation of establishing other activities that we could have received money for such as a food bank. We have seen how other organizations have done so and it has subsequently undermined their vision. So far, we have stayed the line despite the opportunities. But it is a constant struggle and it is very tempting at times.

A staff member of a nonprofit involved in economic development noted the pressures that her organization is constantly under to expand the scope of their mission:

There are always requests for us to be involved in projects that are not necessarily in total sync with our vision. There are things under the umbrella of economic empowerment that pull us from the outside and pull us outside our mission. A government agency asked us to run an employment program for women and we eventually decided that we have to turn down this very appealing offer. While it may help to empower women economically, it does not fall under their own particular vision of helping women to establish their own businesses. We have an ongoing challenge of staying with the organizational vision and we have to be very careful to remember it at all times.

It takes a great deal of discipline, especially for many of these nonprofits that are struggling to secure ongoing funding, not to be diverted from their core mission. Indeed, Wolf (1999) discusses the constant "tug between two competing tendencies" and the frequent dilemma among nonprofits 
of whether to stay within the original mission (despite, for example, lack of funding) or to expand the vision in order to secure organizational survival (p. 27). This "tug" is especially difficult when there is a lack of consensus around the goals and vision of the organization (Wolf, 1999). Especially as an organization continues to grow, a shared 'mental model' that shapes how one views the work and determines a course of action becomes critical (see Section 2.1.1). Creating full alignment between the organization's vision, strategy and capacities is a constant challenge. A staff member of another nonprofit described her experience:

We need to stabilize our growth. It is a difficult transition because we did not invest enough thought in it. We did not want to grow too big but there was pressure: so many people turned to us, the foundations pushed us and the field demanded it. It fits our vision, but the strategy is not controlled by us; the strategy controls us.

How are these nonprofits addressing these issues? Many of the organizations have turned to outside consultants to help them facilitate planning retreats, a common form of capacity building that is generally considered by both staff and board members in the sample as a valuable step in helping to sharpen the organizational vision. The definition of the overall mission, vision and goals, which then serve as the basis for strategy, is an essential process for enabling an organization to establish priorities for action, and to, ultimately, maximize its social impact (McKinsey \& Co., 2001). A staff member of a nonprofit that recently held a retreat talked about what the organization has learned from the experience:

An ongoing discussion of strategy is vital for us. We understood from the retreat that it is necessary to continually ask ourselves questions such as: What is the relationship between the projects? How can the projects support and reinforce one another? How is each project connected to our strategy? Since the retreat we have devoted numerous meetings to these issues.

A board member spoke of the same retreat "We considered strategic planning in the long term, not just per project. We talked about our ultimate, long term goal and asked ourselves what it is that we want for our organization in ten years." 
In a few of the nonprofits in the sample, a turnover in leadership has presented an opportunity for change. For example, one organization operated for many years without any kind of planning according to the budget. As the current director explained,

We used to have one budget for the entire organization. The director took complete responsibility for planning and we never had any of our various projects designed according to a budget. Issues of accountability as to how the money was spent have only recently become an issue and the funders are also demanding this. We now want to make sure that there are only good surprises, not bad surprises. Now the work is designed according to what the organizational budget will allow, and the process is participatory.

Working with a capacity building consultant over the past year, this nonprofit has divided the staff into teams, each of which is responsible for long term planning and the budget of each particular project. There is greater responsibility and accountability on the part of the staff and, not surprisingly, as expressed in interviews, the staff feels much more involved in establishing goals for the organization.

As they cope with various challenges such as pressured work environments and temptations to 'stray' from their mission, nonprofits should be conscious of creating a long-term strategy that is aligned with their vision (McKinsey \& Co., 2001). The following discussion explores this issue further, looking at a key facilitator and a key barrier to developing a shared vision. Among the ten nonprofits, an integration of the organizational mission with the personal goals of the staff is an important facilitator for strengthening the overall shared vision. On the other hand, the unclear role of the board in these nonprofits is a major barrier to ensuring that the organizational vision is aligned with its organizational strategy.

\subsubsection{Key Facilitator to Shared Vision: Integration of Organizational Vision with Personal $\underline{\text { Vision }}$}

A staff member of a nonprofit that was established by university students and works with Arab young adults, made the observation that "There is only a very small gap between our dreams and the dreams of our constituents." In the majority of the nonprofits in the sample, the staff express a very strong personal dedication to the organizational vision. As such, these nonprofits enjoy an important characteristic of learning organizations - personal fulfillment and professional fulfillment are intertwined, and the goals of the staff are consistent and interdependent with the organization's vision. 
According to Senge (1990) "organizations intent on building shared visions continually encourage members to develop their personal visions." Not surprisingly, there is true commitment to an organization's goals when the shared vision reflects one's own personal vision (see Subsection 2.1.2.5.). Several of the organizations are careful to devote time to this process and to consider how the staff members are motivated and inspired to carry out their work. For example, at a nonprofit that works to empower Arab women, the organizational leadership seeks to ensure that every staff member undergoes her own process of self-awareness and empowerment. A staff member explains, "we are regularly encouraged to discuss our own personal views of feminism and to consider how it affects our work here. Our values and assumptions are openly discussed and connected to the everyday." This nonprofit, like many in the sample, recognize the importance of the link between personal and professional fulfillment.

'Shared meaning' is essential to a learning organization; according to Ziegler (1999), members of an organization should strive to successfully construct a 'collective reference point' (p. 56). One of the nonprofits takes the topic of the Holocaust, a subject that is often taboo in co-existence dialogue ${ }^{20}$ as a starting point for a discussion of humanistic values among Jewish and Arab youth. Staff members convey a sense of 'membership' within their organization, as articulated by one of the graduates of the program who returned years later to become a program facilitator. She described her feelings about participating in the workshop and her role at the organization:

The experience changed my world. I became a better person because of my participation in the program. It may not bring peace but it brings peace to the individuals inside their hearts because they are able to learn compassion for the other side ... Now as a facilitator I understand that it is not possible to work with Jews and Arabs who are in conflict and not undergo some kind of internal process. What we require from the participants, we require from ourselves. We build this together. This work places a responsibility on you, that you do not just go home but you live it 24 hours a day. You cannot be a bystander to what is happening around you.

Many of the nonprofits have successfully created an engagement among the staff where individual identity and organizational identity have become tied together. This "spirit" infuses staff members with an energy and a sense of purpose, and is considered a critical piece in helping to ensure the sustainability of an organization (See David, 2000).

\footnotetext{
${ }^{20}$ As explained by a group facilitator of the center, there are sensitivities to the topic because, for the Jews, it is the enormity of the suffering of the Jewish people during the Holocaust, and, for Arabs living in Israel, the Holocaust is associated with the founding of the state of Israel as a Jewish state.
} 


\subsubsection{Key Barrier to Shared Vision: Undefined Role for Organizational Board Members}

Light (2004) discusses fundamental questions that all nonprofits should ask themselves when they are establishing themselves: "How will we make a difference? Who does what in the organization? Why do we exist? How will we know we are successful, if we are?" (p. 141). Among the ten participating nonprofits, there is a question of what concrete role the boards are taking in helping the organizations to ask these types of questions and ensure that there is a coherent vision and strategy.

As part of a movement that is establishing a new tradition in Israel, many of these social change nonprofits' staff, volunteers and board members are learning the dynamics of nonprofits organizations, something that they simply had no exposure to in the past. Several of the nonprofits, when founded, received capacity building assistance in the very essential basics of a nonprofit organization (i.e., the role of the board, organizational structure). The whole notion of 'lay leadership' is a relatively new concept in Israel, and it is especially novel to members of immigrant communities who do not come from a tradition of a thriving nonprofit sector. Not surprisingly, there is often an ambivalence regarding the role of the board among the nonprofits in the sample. There is, for example, minimal if any expectations placed on their board members to take part in the fundraising process. ${ }^{21}$ The legal requirements mandated by the government regarding nonprofit board governance are quite general and there is great variation among each of the nonprofits in terms of board involvement. ${ }^{22} \mathrm{~A}$ capacity building consultant who works with social change nonprofits in the United States and Israel notes that, unlike in the U.S., there are not widely circulated 'standards' in Israel on how a proper board should be functioning (Gantz McKay, 2005). ${ }^{23}$

\footnotetext{
${ }^{21}$ This will be discussed in more detail in the Section 4.5 on 'External Connectedness'.

${ }^{22}$ See Katz, O. (2000) for information about the laws governing nonprofit boards in Israel.

${ }^{23}$ Gantz McKay (2005) cites groups like the Association of Nonprofits in Maryland and in Minnesota that are setting the standard for expected practices among non-profits in the United States.
} 
Often the board's role is to represent the nonprofit organization and to help promote it publicly; however it is often an ambiguous role. One director spoke very frankly about the topic:

Our board is not active, which is a generally the case among nonprofits that I know. In our case, as in many others, the founders became the professional staff instead of the board. After that it is not easy to choose a board that is above you. Essentially you have to invite someone in who can fire you. For this reason, so many of the boards are weak.

When probed, some of the nonprofits in the sample did not have clear standards regarding the board (i.e., the term length for board members, frequency of board meetings). The lack of clarity regarding the role of the board and the procedures can lead to a variety of problems. For example, one nonprofit, with five staff members and only a four-member board, has to reflect on its ability to remain open to a diversity of perspectives and avoid becoming too insular. Another danger is that the board can become 'stacked' with supporters of the director, as recently occurred in another nonprofit that is embroiled in an ongoing conflict between the staff and the director.

Several of the nonprofits are currently working to strengthen the role of the board by increasing its involvement and clarifying board procedures and responsibilities. One nonprofit, which recently underwent a leadership crisis and the subsequent departure of two senior staff members, has greatly benefited from changes in both the make up and level of engagement of the board. According to a staff member, "the previous board approved things that were brought to them, but everything that was brought to them was after the decision had already been made." Another veteran staff member reflected on the previous board; "perhaps they did not have the energy or the strength to argue or they simply wanted to avoid conflict. Either way it was accepted as a given that our former director made all the important decisions basically on her own." The election of new members and the subsequent changes in various rules regarding board governance (for example, there is now a term limit of three years per board member) has empowered the board to a greater degree. A board member considered another one of the many new changes;

We now meet by ourselves once a month, usually without the staff. We have found that when we meet separately, the board members are more inclined to be proactive...We have tried to engage the board and return a balance of power between the staff and board; we are, I hope, more independent.

According to Light (2004), boards evolve as the nonprofit evolves; board members may take time to understand their role and added value to the organization. In one organization that is seeking to 
further engage its board, several of its board members have attended outside workshops on board development and the board has recently decided to begin meeting monthly. A board member reflected on the new changes:

Lots of our board members were heavily involved in the day to day activities of the organization, such as directly supervising the social workers. There are more of us now that are trying to focus on the overall vision, strategy and criteria for success. We have a new chair and things are changing but still the pace is still too slow. I guess because we are there as volunteers and that is the nature of things.

A nonprofit can enhance its development as an organization by "asking the right questions, addressing potential threats, and choosing the right strategies for building organizational capacity" (Light, 2004, p. 140). The board can play a key role in helping a nonprofit to strengthen its adaptive capacity, starting with its ability to help an organization continually shape and reevaluate the overall vision and strategy (see Gray, 1998). As it helps a nonprofit to look beyond the 'putting out of daily fires' (see Bohn, 2001), the board can also help to bring a diversity of perspectives to the organization and ensure that there is a defined learning strategy. It is this next dimension of adaptive capacity, openness and inquisitiveness, that is discussed in the following section.

\subsection{INQUISITIVENESS/OPENNESS}

How does an organization encourage a sense of inquiry and of experimentation among its staff members? Researchers cite the importance of creating an environment of openness in which staff feel comfortable asking questions, raising problematic issues and reflecting on mistakes (Garvin, 2000; Senge, 1990). Overall, the ten nonprofits in the sample are characterized by collaborative work environments that enable open dialogue and feedback. At the same time, these nonprofits need to ensure that, especially in the face of growth, they can provide the staff with opportunities to learn together in order to continuously improve organizational performance and respond effectively to changing needs.

How the openness to diversity of opinion is perceived in the organization can provide a strong indication of whether learning will take place (see Section 2.3.1). According to the results of the questionnaire, staff members generally feel comfortable asking questions, offering alternatives 
and conveying information that may contradict current practices or beliefs. The majority of the organizations scored high in questions such as "We have new ideas coming from all levels of the organization" and "In my organization, staff are encouraged to offer dissenting opinions and alternatives." Respondents were asked on a scale of 1-5 (1: strongly disagree and 5: strongly agree) to rate the extent that they agree with the above statements. The organizational mean for the item: "We have new ideas coming from all levels of the organization" was 4.0, while the organizational mean for the item: "In my organization, staff are encouraged to offer dissenting opinions and alternatives" was 4.3. Similarly, staff gave a high rating to the statement, "In my organization, staff are encouraged to ask 'why' regardless of rank". Respondents were asked to rate the level of frequency (on a scale of 1-5, with 1: almost never and 5: almost always) that "Staff are encouraged to ask 'why' regardless of rank"; the organizational mean was 4.2.

Learning organizations promote inquiry and dialogue, understanding that diverse viewpoints allow an organization to remain flexible, creative and open to innovation (Garvin, 2000; Senge, 1990). For this to happen, learning- oriented work environments support the open exchange of ideas and encourage staff to raise conflicting perspectives (Garvin, 2000; Senge, 1990). There is a shared understanding that it is more important to ask the hard questions rather than avoid controversy. A staff member at one of the nonprofits in the sample touched on this important issue in an interview:

As long as you have the interests of the organization at heart, the leadership is open to suggestions. We can share ideas about work and about the organization, and it is not perceived as an attack or a challenge to power. The open spirit enables this. When you offer alternatives, or even constructive feedback, you feel that it is appreciated. Whether or not everyone agrees is another issue but you can give your opinion and you can actually see how you are able to bring about change.

Respondents were asked on a scale of 1-5 (1: strongly disagree and 5: strongly agree) to rate the extent that they agree with the statement that is reverse coded: "Most attempts to change important things are greeted with resistance". After recoding the results, the organizational mean for this statement was 3.7. Staff members responded in a somewhat ambivalent fashion to the statement: "Any practice or assumption can be challenged, no matter whose it is, or how long it has been in effect". On a scale of 1-5 (1: strongly disagree and 5: strongly agree) to rate the extent that they agree with the statement, the organizational mean was 3.4. 
In an open-ended section on the questionnaire, staff were asked to choose between completing the two following sentences: "Staff would feel more comfortable challenging practices or offering new alternatives if..." or "Staff feels comfortable challenging practices or offering new alternatives because...". A sample of the responses from those who chose to complete the sentence, "Staff would feel more comfortable challenging practices or offering new alternatives if..." is offered below: ${ }^{24}$

- "If we knew that the organization really is able to check its effectiveness and its impact."

- "If I knew more about the work of the staff members."

- "If our director was actually serious about changing the status quo."

A sample of the responses from those staff members who completed the sentence: "Staff feels comfortable challenging practices or offering new alternatives because..." is listed below:

- "It is vital in an organization, it is not possible another way."

- "Since the character of our organization allows for this, and the majority of things that we do are based on discussions among the staff."

- "The director of the organization is committed to the principles of open discussion, selfcritique, and egalitarian structure."

- "There is an open atmosphere and effort to hear the voices of all the staff, even if the ideas are not necessarily accepted as policy."

- "There is an interpersonal atmosphere that is not formal; most of us know each other outside the framework of the organization, and the leadership projects a desire for cooperation."

- "There is working staff environment that is open and there is an atmosphere of equality between the staff."

In considering whether a culture of inquiry is being nurtured, the organizations can ask themselves the question: 'How quickly does bad news travel upward?' (see Section 2.3.1). To create a safe environment for identifying and addressing problems, the organizational leadership needs to model the behavior by expressing a willingness to discuss mistakes and receive critical feedback (Garvin, 2000). Respondents were asked to rate the level of frequency (on a scale of 1-5 , with 1: almost never and 5: almost always) that "Staff openly discuss mistakes in order to learn from them", the organizational mean was 4.0. In an open-ended section on the questionnaire, staff were asked to choose between completing the two following sentences: "Staff would feel more

\footnotetext{
${ }^{24}$ Out of the 30 responses to this item, 5 respondents completed the sentence: "Staff would feel more comfortable challenging practices or offering new alternatives if...". Twenty-five respondents completed the sentence: "Staff feels comfortable challenging practices or offering new alternatives because...".
} 
comfortable discussing mistakes, receiving and/ or giving open and honest feedback to each other if..." or "Staff feels comfortable discussing mistakes, receiving and/ or giving open and honest feedback to each other because..." A sample of the responses from those who chose to complete the sentence "Staff would feel more comfortable discussing mistakes, receiving and/ or giving open and honest feedback to each other if ..." is provided below: ${ }^{25}$

- "If there was more openness and acceptance of criticism."

- "If there was someone that would coordinate the topic of feedback, to sit with each of us and hear about our work and the work of the other staff members."

- "If we would build the staff meetings and work between staff so that a certain amount of time was devoted to the issue of feedback."

Listed below is a sample of responses from those respondents who chose to complete the sentence, "Staff feels comfortable discussing mistakes, receiving and/ or giving open and honest feedback to each other because...":

- "It is vital to the organization and because it is part of the vision of the organization."

- "There is a desire to improve and work together in order to build and be built."

- "There is an organizational culture of feedback and assessment, formal and informal, and that encourages the discussions of mistakes. There is no feeling of sanctions for mistakes."

- "Because if we do not fix our mistakes now, it can continue and hurt others that come to get help; instead of giving help we can harm."

- "Most of the staff receive constructive criticism or give it with the goal of improving our work."

To enhance innovativeness, learning organizations signal to the staff that providing critical feedback and acknowledging problematic issues are actions that are valued (Garvin, 2000; Raelin, 2000). As part of this effort, the organizational leadership sets the tone by being learners themselves and by demonstrating a willingness to recognize their own vulnerabilities (Schein, quoted in Coutu, 2002). This can obviously present difficulties if the leadership does not model the behavior by openly discussing mistakes as opportunities for learning. One nonprofit scored significantly lower than the others on the item "In my organization, staff openly discuss mistakes in order to learn from them" with a score of 2.6 (as compared to the organizational mean of 4.0).

\footnotetext{
${ }^{25}$ Out of the 27 respondents, 8 respondents chose to complete the sentence: "Staff would feel more comfortable discussing mistakes, receiving and/ or giving open and honest feedback to each other if...". Nineteen respondents chose to complete the sentence: "Staff feels comfortable discussing mistakes, receiving and/ or giving open and honest feedback to each other because...".
} 
In the follow up interviews, it became clear that there is an atmosphere of blame and 'finger pointing' that discourages the staff from honestly discussing problems that they may encounter in their work. An interview with the associate director illustrates how the leadership perceives this issue: "we want people to talk about mistakes but not everyone feels comfortable. We ask them 'What do you need not to fail again?' - but people do not always want to evaluate themselves." Only after an organization acknowledges that mistakes can be opportunities for learning, and looks at not only individual but 'systems' problems, will it be able to learn from its past experience and apply its learnings (Senge, 1990).

While nurturing an atmosphere where staff feel comfortable challenging assumptions and discussing mistakes, an organization should also be consciously aware of its 'learning strategy' (Garvin, 2000). Like many social change organizations, the nonprofits in the sample are taking on difficult social problems with limited resources and little ability to sufficiently invest in the professional development of the staff. In general, nonprofits, as compared to for-profit organizations, have fewer resources to invest in skill development, reward staff performance or provide monetary incentives for staff to engage in activities that may enhance organizational learning (McHargue, 2003). As such, these nonprofits have to be very strategic in developing a learning 'agenda' and a shared understanding of what knowledge is needed to successfully pursue the organization's strategic goals.

When asked about overall challenges facing his organization, a director of one nonprofit expressed his concern regarding the organization's ability to absorb knowledge from the field:

I am not sure how much the staff is continually learning, processing new information and seeking examples of similar work in Israel and internationally. I feel that the push for this comes from me. I look for examples and learn from others but I do not do it systematically. We do not do this enough and I am sure that we could learn more from like-minded organizations and the literature and not always reinvent the wheel. ${ }^{26}$

Several staff members of various nonprofits also raised the issue of professional development without prompting during the interviews. It is worthwhile to consider the extent to which these nonprofits are identifying the skills that are required by the staff to effectively carry out their work and enhance the organization's overall capacity. One frustrated staff member reported that "there is no goal of learning and of developing us professionally. We need to spend more time on the

\footnotetext{
${ }^{26}$ The possibility of exchanging knowledge with other like-minded nonprofits will be explored further in the Section 4.5 on External Connectedness.
} 
professional enrichment of the staff and if necessary, then we give up on some of our goals in order to be able to do so." To cultivate adaptive capacity, learning cannot be left to chance. For organizations to remain innovative, knowledge production and knowledge exchange need to continually be an integral part of the part of the organization and the work life of the staff (Davenport \& Prusak, 2000). A staff member at a different organization shared a similar concern about the need to create more learning opportunities for the staff:

We work based on experience, our intuition. We get feedback on what we do but there is not a theoretical way to evaluate ourselves. What we don't know, we don't know. Perhaps there is more that we should bring to our workshops, either from the news or from current research or wherever. We need to have more training for the staff on a regular basis. Maybe a 'learning day' once a month to get outside knowledge to the staff.

According to Volpe and Marsick, (1999), an organization that wants to nurture a sense of inquisitiveness needs to encourage its staff to continually meet new people, experience new challenges and be introduced to new ideas. It is questionable as to what extent the nonprofits are consciously making an effort to provide both learning opportunities and access to resources for skill development. In the following section, the role of the organizational culture in either enhancing or inhibiting a spirit of openness is discussed further. Whereas many of the nonprofits enjoy a culture that values the exchange of ideas over hierarchical status, it is unclear whether their organizational cultures are adapting easily to change and growth.

\subsubsection{Key Facilitator to Openness: Value-Based Organization Culture}

The majority of the nonprofits in the sample, many of whose stated goal is to create a more just and democratic society, are conscious of linking their organizational values to their organizational management style. In general, the staff view themselves as partners in building the organizational vision together through a participatory model of decision making, a model that tends to be favored by nonprofits (Letts, Ryan \& Grossman, 1999). The staff generally take pride in the open, informal spirit that characterizes their organization, where hierarchical staff distinctions are often in name only. This open environment in which staff can pose challenging questions and discuss problematic issues does not come by chance; many of the nonprofits are very purposeful about creating an organizational culture that mirrors the values that they espouse to the outside. Indeed, 
David (2002) argues that "the degree to which it [a nonprofit] is able to articulate, teach and live its core values with integrity constitutes the foundation and structural framework upon which organizational life is built." (p. 2)

How do these organizations 'live their core values with integrity'? During the interviews with staff members, it became clear that a large percentage of the nonprofits emphasize the importance of matching their organizational behavior to their ideology. With a clear articulation of their value set, these nonprofits ensure that the organizational mission is operationalized in the daily life of the organization (see David, 2002). For example, at the nonprofit that promotes civil education among the Russian speaking community, the leadership is deliberate in creating a participatory decision-making structure as part of as a primary value of the organization. The director described their approach:

We are always adapting - the personal traits of the staff and the non-hierarchical structure where everyone can influence policies allow for this. Each voice is equal and this helps enable us to be open to change and remain innovative. These are values that we are promoting outside and we are purposeful about this.

Similarly, at a nonprofit that is involved in humanistic education, a staff member emphasized the connection between the organizational mission and the organizational practices,

The reason why the staff is all comfortable talking about things is the connection to the philosophy of democracy and humanistic thought. We believe in it here. It is what we teach and what we do. People can't work here if they only want instructions on how to do things.

When a group of activists established an organization promoting the economic empowerment of women, they developed a non-hierarchical structure to reflect their values of equality, feminism and empowerment. Together with a very active board, the staff works to ensure that these values infuse all aspects of the organizational structure and culture. Several staff members discussed how they are continually working to match the organizational ideology with the organizational behavior. According to one veteran staff member,

It does not just happen. We have to work at keeping our organizational structure intact and constantly be very aware that we are communicating with one another. It has a disadvantage in that it takes time and you need to understand the role of each one in the organizations. But this model is a good mechanism for preventing arbitrariness: we 
cannot just make decisions but each decision has to be explained and has to have a logic to it. It is built on principles and everyone has to demonstrate leadership for it to succeed.

To ensure that it maintains its non-hierarchical structure, the organization has found it necessary to take a variety of steps over the past year. For example, great effort has been invested in clarifying job roles and responsibilities, and a rotating position as 'staff coordinator' has recently been established. Interestedly, the organization also decided to set up a human resource committee that is made of up several board members and other volunteers who act as 'mentors' to the staff. ${ }^{27}$ While recognizing the ongoing challenges to maintaining its non-hierarchical structure, the staff and board have remained committed to the model. A board member explained,

The organization grew out of feminist ideology and it is still considered as vitally important. It is translated into the practices of our organization. There is a devotion to the values of feminism and equality and to help the weaker population - and this begins at home - these values need to be reflected in the organization.

Are the nonprofits able to maintain a balance between their value-based management styles and performance requirements? A staff member at the nonprofit discussed above remained skeptical of its ability to maximize its social impact under the current structure,

It is nice in theory and seems to be working, but if we grow any further, I am not sure how it is going to work. And we want to grow and there is the potential to grow. The organization is open and willing to change, as long as it doesn't negate basic ideas that it holds on to.

Another organization in the sample underwent a significant transition, redesigning its organizational structure in an effort to enhance overall organizational performance. Originally founded as a collective that promotes the status of Arab women, the nonprofit had embraced a non-hierarchical structure based on its ideological values of full equality. Two years after its establishment, the board determined that, in order for the organization to continue to develop, it needed a director with the authority to take initiative and make decisions. Indeed, the nonprofit literature has cited the experience of organizations that have 'outgrown' their flat leadership structure and have found that more management was necessary in order to boost organizational effectiveness and performance (Light, 2004).

\footnotetext{
${ }^{27}$ This will be discussed in further detail in the Section 4.4 on Social Capital.
} 
As these nonprofits continue to grow and develop, they are often reassessing their values in the face of change. At times, the organizations struggle to balance their organizational culture with the demands for innovativeness and growth. While the values of a participatory structure can serve the organization well in maintaining a diversity of opinion, the organizational 'organic roots' (see Light, 2004) can be challenged when there are demands for change, as is discussed in the next section.

\subsubsection{Key Barrier to Openness: Balancing their 'Organic Roots' with Growth}

All of the nonprofits in the sample have been established over the past ten years and are now reaching similar stages of development. A large number of these organizations have successfully implemented their model or "program theory" 28 and are continuing to grow in size and scope. New areas of specialization are being developed and organizations are creating follow up projects for the graduates of their programs. Other organizations are reassessing their strategies; several of the nonprofits, for example, that primarily focus on education are adding advocacy to their activities. $^{29}$ What is apparent among many of these nonprofits is their ability to continue innovating in new directions while remaining within the framework of the organizational mission. At the same time, however, many staff members reveal a sense of ambivalence regarding the growth of their organization. These nonprofits can ask themselves how their organizational culture and connection to their 'organic roots' impact on their ability to continually adapt and innovate.

Light (2004) writes of the "the spiral of sustainable excellence" and refers to five 'stops ' along the way: "the organic phase, the enterprising phase, the intentional phase, the robust phase and the reflective phase." 30 Several of the nonprofits in the sample seem to be encountering difficulty in

\footnotetext{
${ }^{28}$ For more information about program theory, see Chen (1990).

${ }^{29}$ As the director of an organization that promotes a civil society explains, "The educational investment is a long term goal, but the reality requires action now. The need is too urgent."

${ }^{30}$ Considered as a spiral, where organizations can move up and down the spiral at any time, Light (2004) defines the five stages of organizational life: "the organic phase of life, in which they struggle to create a presence in their environment; 2) the enterprising phase, in which they seek to expand their size and scope; 3) the intentional phase, in which they become focused more tightly on what they do best; 4) the robust phase, in which they strengthen their organizational infrastructure to hedge against the unexpected; and 5) the reflective phase, in which they address longer-term issues of succession and legacy." (p. 136)
} 
moving from the 'organic phase' to the 'enterprising phase' of development. For example, the board member of one nonprofit voiced his strong reservations about the organization's rapid expansion: "We have had too much growth and there is great concern that we will not be able to hold onto our success." A staff member of another nonprofit expressed her concern regarding growth: "We are a small organization doing big things. And you can lose important things when trying to be big." A board member of the same nonprofit articulated similar sentiments:

The strength of our organization is the connection to the goals and vision, and it is comprised of people who bring with them a true ideology and commitment. I fear that as we continue to grow -we will lose this naivety.

What are some of the difficulties that the nonprofits are encountering in the midst of growth? A senior staff member of an organization that grew from seven to fifteen staff members in less than three years reflected on the changes,

We used to have one big space, with a backyard and trees, where everyone would hang out. Since we have moved to this office building we are more separated from each other and have no natural meeting place. When we were smaller, everyone got their hands dirty and we didn't make distinctions between the roles of the staff. It is much different now. Now I feel much more separation.

Even seemingly minor issues can present difficulties for these nonprofits. At one nonprofit that is heavily based on volunteer activity, there is an ongoing discussion of whether it is necessary to call the volunteers by phone or if email notices can suffice. Another nonprofit has reluctantly come to the conclusion that it needs an administrative assistant - a complex decision because it clashes with the image that the organization holds for itself. A staff member explained, "We have an informal, not 'office-like' kind of organization. We have a spirit of looseness and freedom. We want to make sure that the first introduction to our office is not through an administrative assistant."

Several of the nonprofits, originally locally based, are coping with the pressures of 'going national'. One of the nonprofits is currently seeking to open a branch office in another part of the country. A board member considered the difficulties,

We are part of a small network and we are able to receive informal feedback on our programs and on the individuals associated with the organization. We now have to cope with the pressure to expand. There are challenges of where to open a new branch and if it is possible to find people who the organization can trust. 
Another nonprofit, which has grown from a budget of $\$ 30,000$ to $\$ 450,000$ in less than five years, is also seeking to effectively manage its growth. The organization has recently determined that it does not want to grow further but concentrate on stabilizing itself. A staff member reflected on the changes:

We started out at as a small group meeting in each other's homes and now we are working all over the country. But there is a price. We are no longer directly connected to the women. We train the facilitators and develop the training... Now as we get bigger we need to be an institution, more bureaucratic - it makes it feel less personal... We are not spontaneous anymore.

Similarly, the director of another nonprofit who is working with an outside consultant talked about recent changes that are taking place at her organization: "We now have the challenge of moving from 'ad hoc' procedures that were based on ideology and commitment into one that has established roles, and yet at the same time allows flexibility."

Letts, Ryan and Grossman (1999) write of the fear of 'bureaucratization' that nonprofits often see as presenting a threat to their values and organizational culture (p. 53). As indicated in the interviews, the nonprofits in the sample are having difficulties in formalizing processes and systems that may have been less critical prior to their recent growth. Many of these nonprofits are searching for the balance between an organizational culture that prizes informality and openness and the need to establish systems and structures that can provide stability and enhance learning.

As a large number of these nonprofits enter into a new phase of their development, it is essential for them to put systems and processes in place and provide time and space for their staff to exchange knowledge, learn collectively and work in cooperation. Whereas in the past the sharing of knowledge may have come naturally and on an informal basis, these nonprofits need to ensure that both tacit and explicit knowledge is 'institutionalized' in formal mechanisms as well (see Garvin, 2000). Especially when an organization is small, its resources (i.e., staff, finances) determine, to a great extent, the overall organizational capacity (Christensen \& Overdorf, 2001). When an organization grows larger, its capabilities are much more defined by its 'process', a transition that is referred to by Christensen and Overdorf (2001) as "the migration of capabilities" (p. 111). These nonprofits need to ensure that the processes - "the patterns of interaction, coordination, communication and decision making" are well articulated and enable the organization to continually learn and nurture a spirit of innovativeness (Christensen \& Overdorf, 2001). 
One of the nonprofits in the sample stands out as particularly deliberate about institutionalizing its knowledge strategy and making it an integral part of its 'processes'. This organization 'knows what it knows' and ensures that this knowledge is the 'common property' of the organization (see Section 2.3.1). Since its establishment, the director and staff have been meticulous about documentation and meeting protocols. ${ }^{31}$ As a built-in part of the process, the staff sets time aside for reflection upon the completion of each workshop. Then, during the planning phase, the staff reviews the 'debriefing' notes and incorporates its learnings into the design of the workshops. This organization, like all learning organizations, understands that as Garvin (2000) writes, "no activity is truly complete until participants have reflected on their experiences and understood the reasons for success or failure. Then and only then, has learning been incorporated into daily work" (see Subsection 2.2.2.5).

Whereas some of the nonprofits have a well-conceived learning strategy, other organizations in the sample often seem to be struggling to manage their knowledge and make sure that it 'moves' through the organization. Especially as each nonprofit continues to grow, it should ask itself whether it openly articulates its knowledge and informational needs. In essence, organizations need to continually pose the questions: Do we have a defined learning agenda (Garvin, 2000)? Is it an integral part of our organizational 'system' or it is left to chance? The systematic collection and distribution of knowledge is further explored in the following section.

\subsection{SYSTEMS/ EVALUATIVE THINKING}

The intimate and informal nature that characterized the establishment phase of most nonprofits in the sample is slowly being transformed, either intentionally or unintentionally, in order to cope with growth. Many of these nonprofits organizations are now finding that, as they move into a new phase of development, it is necessary to have very clear systems in place for learning and for evaluating performance. In order to continue to enhance their adaptive capacity, they need "the

\footnotetext{
31 As a counterexample, another nonprofit in the sample does not document any of its meetings. In the already contentious environment that exists in the organization, the lack of documentation only serves to exacerbate the problem. There are constant disagreements about lines of responsibility and accountability. Decisions that are made in meetings are not recorded, which inevitably leads to disagreements about what was decided. With specific regard to the director, staff are unable to 'protect' themselves with proof of documentation at a later stage when the director capriciously changes his mind or alters a decision that was made in a meetings.
} 
skill to take the initiative in making adjustments for improved performance, relevance and impact" (Sussman, 2003, p. 1). An essential component of adaptive capacity is the ability of the staff to have an overall understanding of the relationship among the various parts of the organizational 'system' and to systematically evaluate their work. It is an ongoing challenge that organizations are struggling to cope with effectively.

Systems thinking is essential for seeing patterns of change versus static 'snapshots' and for understanding how parts of the 'system' constantly interact with each other, often creating unpredictable change (Senge, 1990). In order to gain insight into how these nonprofits are equipping themselves with a framework for understanding the interrelationships between various aspects of the organization, staff members responded to items in the questionnaire such as "In my organization, staff are very conscious of how changes in one part of the organization can affect other parts" and "When new information that would be helpful to others is learned or discovered, it is shared with those in the organization who need it ". Respondents were asked to rate the level of frequency (on a scale of 1-5, with 1: almost never and 5: almost always) for the two questions cited above. Staff members gave their organizations relatively high marks: the organizational mean for both questions was 3.9.

To effectively evaluate organizational 'systems', it is necessary to have the ability 'to stop and look below the surface, to see how the structure (the relationship between the parts of the system) drive the patterns of change we see" (Sweeney, 2001, p. 31). Are nonprofits, chronically underinvesting in their organizational capacity, able to sufficiently take time "to stop and look below the surface"? "Nonprofits always serve their mission first," which often results in pressured work environments and overburdened staff (Light, 2004, p. 14). This type of work environment can create a culture of 'fire fighting' (Bohn, 2001), a problem that was raised, for example, by a staff member of one of the participating nonprofits:

The problem here is the inability of our organization to do anything that is not in 'crisis mode'. Our time is constantly stressful; people's individual time is not at all respected. Because of the lack of organization, we have to work frantically, come in during the weekends and take care of the big crisis. Then it just starts all over again.

What happens when organizations are constantly in a "crisis mode"? Bohn (2001) writes about the culture of 'fire fighting' in organizations, defining it as collection of symptoms that also can represent a chain of events. 'There isn't enough time to solve all the problems; solutions are 
incomplete, problems recur and cascade, urgency supersedes importance, many problems become crises and performance drops' (p. 163). It is questionable how these nonprofits, understaffed and with few resources, are always able to ensure that they are not jumping from crisis to crisis and not just temporarily solving problems until they recur again. Under pressure, it is easier to react to events and respond with immediate action (the mentality of 'fire fighting'), than to take the time to reflect on how the event fits into a larger pattern. Our 'mental models' already have "short term horizons", and therefore we naturally have a tendency to overlook the underlying causes of events and unintended consequences (Sterman, 2001). By forgetting that the system is dynamic, unpredictable and governed by its own feedback, 'fire fighting' can end up working against the desired outcome or simply shift the problem to a different part of the system or into the future.

Systems thinking requires analysis of multiple perspectives and on multiple levels in order to gain an understanding of how the relationships between the parts of the system constantly interact and create unpredictable change (Sterman, 2001; Senge, 1990). As part of an effort to adopt systems thinking, it is important to expand one's mental models, focus on feedback, and seek multiple points of view (Sterman, 2001). One nonprofit, for example, takes advantage of an important opportunity to receive feedback on its operations and processes. An Arab youth organization regularly hosts European exchange volunteers and international trainers for an average period of three months. Towards the end of their visit, the volunteers or trainers are asked to write an evaluation and develop recommendations for the nonprofit together with the staff. A staff member talked about the value of an outsider's perspective on the implementation of organizational 'systems':

The outsiders have a more objective view of the organization. It gives us the opportunity to see how others view our work, how we communicate with one another, how we handle stress, if we follow up properly.... In return, they understand that they are important to us and that they have contributed to further developing the organization... We do not see ourselves in the final space ( sic). Rather we take the attitude that we are not good enough; we want to improve all the time."

A fundamental component of an evaluative attitude in an organization concerns the desire to "improve all the time". Especially for nonprofit organizations that are founded to respond to a community need, it is critical to continually incorporate outside feedback and to evolve in response to changing circumstances. Using evaluation to plan, measure progress, and assess 
community needs is essential for ensuring that a nonprofit is relevant to its constituents, effectively meeting its desired outcomes and fulfilling its mission (Sussman, 2004; Gray and Associates, 1998).

As it became apparent in the interviews, staff members from those nonprofits with a "culture of inquiry' and a defined approach to evaluation have, not surprisingly, a very clear understanding of their evaluation strategy. Over half of the nonprofits have an evaluation plan that incorporates the use of surveys, focus groups or other forms of eliciting feedback from stakeholders. Staff members of nonprofits with strong evaluative processes referred to it as, for example, something that "is obvious to everyone that that is a station in the process" or "as an integral part of the work." Yet, views on evaluation, more so than the other aspects of adaptive capacity, often diverge among the staff members of nonprofits in the sample. Within the same organization, for example, there are often inconsistent views on whether evaluation is being carried out, and if so, if it is being done satisfactorily. ${ }^{32}$ In an interview, the director of one nonprofit spoke about the importance of evaluation: "It gives us an ability to see the outcome. Each day is so intensive; evaluation makes us stop and look and say "wow, we did it." Yet a board member of the same organization confided, "we have not yet defined our criteria or decided what to check, or what are the indicators... Without criteria, it is difficult to know."

Like many nonprofits, the organizations in the sample are tackling difficult social problems where progress is both hard to make and challenging to measure. As indicated in the interviews, the nonprofits often encounter difficulty in defining and measuring outcomes of the work. To further complicate matters, there tends to be a variety of interpretations regarding the purpose, the process and the use of evaluative results. While the questionnaire results offer some insight into these issues, they also point to the degree of ambiguity regarding evaluation. ${ }^{33}$ Respondents were asked on a scale of 1-5 (1: strongly disagree and 5: strongly agree) to rate the extent that they agree with the following statements regarding evaluation. For example, the organizational mean for the statement: "Information is gathered from stakeholders to gauge how well we're doing" was 3.6. The organizational mean was 3.9 for the questions: "Currently available information tells

\footnotetext{
${ }^{32}$ In the interviews and questionnaire, the process of evaluation was generally defined as "the systematic collection of information in order to better understand the programs, improve effectiveness and make decisions about future planning."

${ }^{33}$ The impressions of the researcher is that there is not necessarily a correlation between the organizations that received 'high scores' and the actual existence of a strong evaluative strategy. Organizations that have the most sophisticated evaluative mechanisms did not rate themselves higher than nonprofits that are lacking a clear evaluation plan.
} 
us that we need to know about the effectiveness of our programs." ; "In my organization, staff continually ask themselves how they're doing, what they can do better, and what is working". Another question that was reverse coded, "Evaluative activity is seen as threatening the status quo", also received an organizational mean of 3.9 after recoding.

In the open-ended section of the questionnaire, respondents were asked to choose to complete one of the following sentences, "We would carry out more evaluation if..." or "We carry out evaluation because...". Many of the respondents who chose to complete the sentence "We would carry out more evaluation if..." cited time or financial restraints. A sample of the other responses is provided below: ${ }^{34}$

- "If there were tools to evaluate the work of the staff."

- "If there was a more clear methodology."

- "At this point, we need to establish a structured evaluation procedure, and to have someone in charge of this process - at this stage, we don't have staff/time to focus enough attention on this."

- "If it was possible to check the changes that we are striving for."

- "If we were aware of its importance to the improvement of the conditions of the organization."

A sample of the responses from those who chose to complete the sentence, "We carry out evaluation because..." is presented below:

- "We want to improve our work all the time, and contribute the best to our target population and therefore systematic evaluation is considered of great importance."

- "It contributes to understanding how we are doing and enables us to reach conclusions about the future."

- "It is built in and institutionalized, and it is accepted by everyone as an organic part of the work."

- "This is the way we work."

- "This leads to identification of relevant needs that make us more efficient."

- "Without this, it is difficult to plan the work and to check the level of success and the need for change, if indeed there is a need for change."

While many of the staff indicated the value that is placed on evaluation, it is questionable as to what extent evaluation is integrated into their planning processes. A notable exception is the

${ }^{34}$ Out of the 27 respondents who answered the question, 15 respondents chose to complete the sentence, "We would carry out more evaluation if...". Twelve of the respondents chose to complete the sentence "We carry out evaluation because...". 
nonprofit that brings together Jewish and Arab youth for dialogue around the Holocaust and humanistic values. The director discussed the approach to evaluation:

We have been evaluating ourselves from the very beginning. Because of the sensitivity of our work we need to be sure that we are impacting positively on our participants and not the other way around. At first we had a professional, external evaluation to check our work and give us feedback on how to improve. Now we have learned how to do our own surveys and focus groups and it has become part of the system of how we do things.

What is more common among nonprofits is to identify a societal need that is not being met and, in an admirable way, jump right into the 'doing'. With a strong commitment and little resources, nonprofit staff often develop a "just do it" attitude that places a priority on the 'service' and not on the 'analysis' or 'measurement' (Letts, Ryan \& Grossman, 1999, p. 33). Frequently during these early stages of organizational development, key decisions are not made about how to track impacts and account for time and money (Light, 2004). Indeed, many of the nonprofits in the sample began evaluating themselves only at a later stage, often at the instigation of the funders. There is not necessarily an awareness that evaluation should be built into the process from the early stages of program development (e.g. needs assessment, formative evaluation; see Patton, 2002). For example, this attitude is reflected in an interview with a staff member at one nonprofit, established five years ago, that is currently developing evaluative tools to measure the impact of the program on its participants.

What enables this is that we are at this particular stage of development - at the point where can that do this. Before we were still formulating the model, networking, etc. We are in a process of growing and developing our strategy and we realize we are at a point where we need to evaluate our work thus far in a honest way in order to take steps forward. It is about timing and about motivation. We are not just giving it lip service... The obstacle is that there are more pressing concerns. But funders are asking about definitive outcomes and we do not have a clear system for measuring outcome.

Rather than perceiving evaluation as a luxury that is only possible after a certain stage of development, learning organizations ensure that evaluation is an integral part of the planning as well as the implementation of organizational activities (Preskill \& Torres, 1999). The staff members of the nonprofits in the sample, however, face numerous challenges in developing and executing an evaluation strategy such as time constraints, minimal resources, and little experience with formal evaluation. While the staff may be have a strong motivation to make a social impact, it is necessary to consider how much the evaluative process of regularly collecting and 
interpreting information to inform decision making has become an integral part of the organizational culture. In the next two sections, a key facilitator and a key barrier to the creation of an effective evaluation strategy are discussed in depth.

\subsubsection{Key Facilitator to Evaluative Thinking: Desire for Impact}

While some of the nonprofits in the sample may lack the ability or the knowledge to effectively carry out systematic evaluation, the majority of staff at these nonprofits displays an obvious commitment to their organization's vision. There is a genuine connection to the goals of the organization and therefore a strong motivation among the staff to consider the impact of their work.

As indicated in the interviews, staff members of the nonprofits, especially those working directly with a defined 'target population' ${ }^{35}$, tend to place a high value on the feedback received. For example, a staff member of an organization that was established by Ethiopian university students discussed the use of evaluation in the home visitation program to newly arrived Ethiopian families,

We know our community well and we are always looking for more feedback informally and through monthly surveys with the families. It is absolutely essential in our work. We need to make sure what we are doing is helpful to the families and not causing any harm.

A staff member at the women's economic independence group expressed a similar opinion towards evaluation that is shared by other staff members at her organization, "If evaluation does not prove that our existence is making a difference, then there is no point that we are around."

With such high levels of personal commitment among the staff, as indicated in the interviews, a key aspect of their professional satisfaction is an overall sense that they are making some kind of impact. Letts, Ryan and Grossman (1999) write that "the problem of burnout, so prevalent among nonprofit employees, is caused not only by difficult work, or even lots of difficult work, but by difficult work that does not seem to produce results" (p. 124). Since so many of the nonprofits are addressing problems that will take years to achieve measurable social impact, it is important for their staff to see how they are advancing their organizational mission. The staff in the sample clearly tend to be motivated by seeing how their work achieves visible results, and

\footnotetext{
35 Those nonprofits working in advocacy, for example, often have a much larger and less defined target population.
} 
because they are often in close contact with their target population, they can receive immediate feedback. For example, a staff member at the women's shelter reflected this sentiment:

We are operating in emergency mode all of the time. The women will leave here if we do not plan ahead and make it work for them. Otherwise they will return to their husbands so it is essential to plan and look at our programs and think about how to make it workable for the battered women and their children. We all really want to make a difference in the lives of these women.

Systematic evaluation that allows a nonprofit to track its progress and to better understand how to enhance its performance can also help to improve the level of job satisfaction and motivation among its staff (see Lafleur, 1995 or Fetterman, 2001).

Still in the early stages of development, some of the nonprofits in the sample lack an evaluative strategy and have yet to make the distinction between structured and informal feedback. For example, one organization working with the gay and lesbian community has operated for years on a volunteer basis and has only recently received funding for a part time coordinator. Yet even though they have not begun to carry out systematic evaluation, there is an ongoing effort to learn about the needs of their community by monitoring their programs through participation levels and ongoing feedback from the intimate network.

Everyone who is here volunteers with all of their heart. They feel discriminated against in their lives and want to do something about it. We are constantly asking people, inviting them in and trying to build this together. ... Because we are such a small community, we receive direct feedback on our events. We are also always getting suggestions on how to shape the programming. We are here for the community, not for ourselves and we are always flexible based on the feedback.

Some of the nonprofits have used creative ways to stay in touch with their target population and continually assess the needs of the community. For example, the organization that works with the Russian-speaking community started a hotline that would operate for a few hours each week. Originally planned as a three-month project, the hotline was designed to enlist feedback and gain insight into the issues facing the community. Due to overwhelming response, the hotline eventually became a regular part of the operations and another tool for civic education and outreach.

While the staff in the nonprofits in the sample clearly demonstrates a strong conviction and desire to make an impact, they do not always have the skills or knowledge to create an effective evaluative strategy. According to the data, it is not always clear to what extent staff members are 
basing their assessments of organizational success on systematic evaluation versus intuition. It is also important to consider how the organizational culture supports the measurement of performance, a topic that is addressed in the next section.

\subsubsection{Key Barrier to Evaluative Thinking: Gap between Organizational Culture and Performance Culture}

As they balance their 'organic roots' with demands for change and growth, many of the nonprofits in the sample are implementing evaluative systems and seeking ways to enhance their overall effectiveness. Yet, at the same time, it is important to ask questions such as whether evaluation is genuinely viewed as an activity that supports organizational learning or if it is driven by external or internal demands for accountability. The following discussion briefly considers how the organizational culture of some of the nonprofits in the sample may inhibit their ability to evaluate themselves and take actions to improve performance.

One of the organizations, for example, was founded by university students who worked for years as volunteers prior to the organization's official establishment. Five years later, its 'organic roots' as volunteer-based and volunteer-driven still permeates the organization. A staff member explained,

From a truly grassroots, volunteer-oriented organization, with a few, very committed paid staff and very dedicated volunteers, we are moving into a new phase now. There is more staff and we are less and less reliant on volunteers. We need to move to another level where the work is different and the expectations need to be higher.

While this nonprofit has a very strong value system, it does not have the history of a reporting culture among the staff that is now required. The staff members, after years of operating in a work environment that was "volunteer-oriented", now need to be held much more accountable for gathering data, documenting their experiences and sharing their learnings.

A critical element in creating a 'culture of inquiry' is not only the establishment of a data collection and measurement system, but also an organizational culture where the process of selfevaluation is valued (see Section 2.4.6). The organization cited above is struggling to create a new culture of reporting and require greater accountability. As the staff member cited above continued to explain: "we are less effective because of our niceness - we do not put our foot 
down enough, but it is changing." Similarly, the director of another nonprofit spoke of the difficulties surrounding a recent effort to encourage staff members to fill out their weekly schedules on a wall calendar at the office. As the organization continued to grow, it was determined that a wall calendar would enhance communication, encourage knowledge exchange and transparency. Yet, after years of operating without any kind of reporting procedures, staff are balking at the effort to account for their time on a regular basis.

In the early stage of their development, a stage often characterized by instability and uncertainty, a number of these nonprofits encountered difficulty in institutionalizing sound management processes or evaluative systems. Many of these nonprofits, now moving up the spiral of development (see Light, 2004), are currently more focused on implementing processes and standardizing procedures that can ultimately enhance performance. One organization, established over five years ago, is changing. "Before everything operated on oral agreement and now we are moving to written, outlined procedures and contracts, and even writing down the number of allocated vacation days or sick days." A veteran staff member explained,

We needed to strengthen the procedures and practices in the organization. Originally, when we were just starting, things were free and equal. There were things that were unwritten but understood- there was an informality to everything. Now we are moving to a more formal phase; we are using reports, setting procedures on how to use money, writing contracts with the staff, etc.

While trying to hold on to elements of its "free and equal" culture, this nonprofit understands that it needs to adapt itself in order to improve its overall effectiveness. It is a challenge that many of the organizations in the sample are struggling with as they move from the 'organic' phase into the 'enterprising' phase of development (See Section 4.2.2). Establishing assessment procedures are especially difficult when there are not any systems in place to help the staff understand how it is possible to evaluate their work. One nonprofit, for example, has not yet articulated how 'success' is defined and there is no shared understanding among the staff on how to measure performance. The staff, which is currently reassessing its operations with an outside consultant, has identified a long list of problematic issues. For example, there is no standardized way to train a new staff member; there are no job descriptions on file, and there are few guidelines for carrying out projects. A staff member explained, "There is an unspoken hierarchy between veteran staff and new staff. We don't receive any regular feedback during the process. Then at the end, under pressure, the director or the more veteran staff members come in to 'save' 
the project." Staff has a difficulty understanding what are the expectations of the work. Another staff member remarked, "It is not that I just don't know what others do, I don't know what I am supposed to do."

Many of the nonprofits in the sample with this 'just do it' attitude have not invested time in clarifying the basic expectations about governing the organization such as reporting routines, staff responsibilities and core administrative systems. With this ambiguity, staff has more difficulty understanding what the expectations for performance are, where they should invest their time, and how they can measure their work. Organizations can continue to achieve program impact under these conditions, but they are not achieving maximum performance and will be unable to sustain themselves over time (Light, 2004). Furthermore, a lack of clarity and shared understanding around work procedures and organizational operations can damage the social capital in the organization, a topic that is discussed in the following section.

\subsection{SOCIAL CAPITAL}

According to Cohen and Prusak (2001), we require crucial elements of social capital in the workplace; we have "the same social needs and responses as other parts of our lives: the need for connection and cooperation, support and trust, a sense of belonging, fairness and recognition" ( $p$. $\mathrm{x})$. For these nonprofit organizations, their stock of social capital and the staff's engagement with the work and with one another is critical. Similar to the staff of other nonprofits, the staff of these organizations are tackling very challenging social problems, and are generally doing so in high stress environments under less than ideal working conditions and with comparatively low compensation. There are certain factors that contribute to the high level of social capital in these nonprofits such as the collaborative work environments and overall commitment to the organization's vision. At the same time, these nonprofits can consider whether they are proactively creating social networks among staff, signaling the importance of knowledge sharing, and establishing organizational policies that nurture a sense of trust.

Social capital is an essential component of becoming a learning organization; knowledge creation and exchange are enhanced when staff feels a sense of trust and belonging to the organization (Cohen \& Prusak, 2001). Informal learning, a key aspect of knowledge production, 
takes place when staff is fulfilled both personally and professionally (Volpe \& Marsick, 1999). A 'sense of community' in an organization provides support to staff members, while positively impacting on their level of motivation (see Subsection 2.1.2.4). For example, as a staff member of one of the nonprofits in the sample who works with battered women explained, "We are part of a very difficult environment. We are dealing with neglect and violence, trauma and tragedy all the time but you can be here and give what you can because of the support you receive." Similarly, a staff member of a nonprofit that facilitates dialogue between Jews and Arabs discussed the challenges of working on complex social and political issues: "Due to the nature of our work, we are always dealing with dilemmas, and it is very difficult in the current reality. We have to continue on with our way despite what happens. This is the place to get a hug and then continue on."

The features of social capital include the "networks, norms and trust that facilitate coordination and cooperation" in an organization (Putnam, 1993, p. 1). Accordingly, the manner in which an organization handles conflict and encourages dialogue are important indicators regarding its level of social capital. The nonprofit staff in the sample were asked to answer a variety of items in the questionnaire that touched on these issues. Overall staff members gave their organizations relatively high scores in these domains. Respondents were asked on a scale of 1-5 (1: strongly disagree and 5: strongly agree) to rate the extent that they agree with the statement, "We have fruitful, constructive debates about new ideas." The organizational mean for this item was 3.9. For the statements "In my organization, whenever staff state their views, they also ask what others think"; "In my organization, staff are resistant to having their work critiqued by someone else", and "In my organization, staff face conflict over work issues in productive ways", respondents were asked to rate the level of frequency (on a scale of 1-5, with 1: almost never and 5: almost always) that this occurs in their organization. The organizational mean for the item "In my organization, whenever staff state their views, they also ask what others think" was 4.3.and for the item, "In my organization, staff face conflict over work issues in productive ways" was 3.6. The organizational mean for the reverse coded statement "In my organization, staff are resistant to having their work critiqued by someone else", after recoding, was 3.7.

In the open-ended section of the questionnaire, respondents were asked to chose between completing the sentence: "Staff would face conflict over work issues in productive ways if ..."or 
"Staff faces conflict over work issues in productive ways because...". A sample of those who chose to answer "Staff would face conflict over work issues in productive ways if..." include: ${ }^{36}$

- "The relationship between the staff was better."

- "If we would have a fruitful dialogue on the source of the position of each one of the staff."

- "If loyalty to the director was not the only thing rewarded here."

- "We were less fearful of a blow up."

A sample of responses who completed the sentence "Staff faces conflict over work issues in productive ways because..." are as follows:

- "The friendships we have."

- "Conversations and willingness to listen to opposing opinions."

- "Maturity, experience, supportive organizational environment."

- "Open dialogue, personal conversations, staff meetings, and teamwork."

- "The evaluation of the organization and projects, weekly staff meetings, strategic planning and social activities for staff and board, an atmosphere of honesty, maturity and loyalty."

In order to continually enhance the learning process, the organizational leadership seeks out multiple perspectives and does not shy away from potential conflict that may arise as a result. Because knowledge is dynamic, collective and 'interactive', dialogue is critical in a learning organization (Ziegler, 1999; Senge, 1990). An organization that enjoys high levels of social capital will benefit from the process of collective learning that takes place with open dialogue, where the sharing of knowledge ultimately leads to the construction of new understandings. For example, one of the nonprofits, under new leadership in the past year, is proactively working to manage conflict and has an outside facilitator present at all staff meetings. As one staff member explained,

There is a lot of tension and the facilitator allows us to work through it together. There is a place for it now, a 'space' to speak out about it, and this helps to reduce the tension and build up the trust between us. Now there is no subject that we can't talk about it. Conflict still exists, and it can still be unpleasant, but there is no longer such a thing as 'forbidden' and the staff feel more comfortable contributing, talking about mistakes and giving new ideas.

\footnotetext{
${ }^{36}$ Out of the thirty respondents to this item, five respondents chose to complete the sentence: "Staff would face conflict over work issues in productive ways if ...". Twenty five respondents chose to complete the sentence: "Staff faces conflict over work issues in productive ways because...".
} 
Another staff member expressed similar sentiments,

The director encourages these discussions and promotes an environment of open dialogue. Unlike in the past, there is not fear that if you express yourself it will affect negatively your position or status. With the help of the facilitator, we are addressing the tensions. We know that without disagreements it is impossible to move forward.

This organization has, to paraphrase Senge's (1990) words, allowed conflict to become "part of the ongoing dialogue" (see Section 2.3.1).

An organization with a high degree of social capital can easily tap into the collective nature of knowledge; staff feel comfortable exchanging information and working together cooperatively (Cohen \& Prusak, 2001). A network of relationships that is characterized by trust is an important factor for supporting cooperation, and, ultimately, organizational learning. Among the nonprofits in the sample, staff members cite numerous opportunities for cooperative work. Staff generally perceived cooperation and team work as critical components of their work. For example, in the interviews, staff made comments such as "the majority of the work is done in teams" or "teamwork is our method of working- every product is the fruit of cooperation."

In the questionnaire, staff members were asked on a scale of 1-5 (1: strongly disagree and 5: strongly agree) to rate the extent that they agree with the statement, "We tend to work collaboratively with each other...". Respondents gave their organization a relatively high score on this item, with an organizational mean of 4.1. In the open-ended section, staff members were asked to choose between completing the sentence "Staff would work more cooperatively together and share more knowledge if..." or "Staff works cooperatively together and shares knowledge because...". A sample of responses by those who chose to complete the sentence, "Staff would work more cooperatively together and share more knowledge if...". 37

- "If there was a better definition of the role of the staff."

- "If there were more meeting points between the majority of the staff. Currently it is spread out and part time."

- "If we succeeded in planning more days that all the staff could be present."

- "If there was not power struggles or ego issues."

- "If we would know better how to manage our time."

37 Out of the 32 responses to this item, 13 respondents chose to complete the sentence "Staff would work more cooperatively together and share more knowledge if...". Nineteen respondents chose to complete the sentence: "Staff works cooperatively together and shares knowledge because...". 
Those respondents who chose to complete the sentence, "Staff works cooperatively together and shares knowledge because..." raise a variety of issues that contribute to the stock of social capital in the organization. For example,

- "Because this is the way a better evaluation and mutual feedback occur."

- "It is part of the ideology of the organization."

- "There are good personal relationships among the staff. There is high motivation and an absence of competition, open or hidden."

- "There is an understanding that the more information that flows, the more efficient the organization will be."

- "There is no choice. We need this in order to carry out our work most effectively. And we are interested in cooperative work."

- "We respect each other's different backgrounds, and we need each other."

While many of the nonprofits are characterized by an atmosphere of trust and cooperation, there are numerous challenges they face in nurturing social capital. For example, one of the difficulties often cited by staff is the logistics of coordinating time together when such a large percentage of the staff work part time. With varying work schedules, communication among the staff becomes especially vital. A staff member at one of the nonprofits emphasized its importance:

We need to be very intentional and proactive in ensuring that the staff is able to connect, to plan, and to reflect on things together. Most staff works part time, three days at most. The only way to work as an organization is to have a high level of cooperation where all of us know what is going on... We consciously keep everyone on the ship because objectively we have little time that we are all together.

At another nonprofit that has grown over the past year, the regular weekly staff meetings were gradually turning into monthly staff meetings. Several staff members expressed concern about the fact that the weekly staff meetings were no longer taking place. One staff member explained, "We are so pressured with work and I miss the discussion at staff meetings. It is a process that is very encouraging for me and it is lacking." Another staff member discussed the problem of irregular staff meetings, "Learning is encouraged here, but the work in the field competes with this. The demands in the field always take precedence and our staff meetings have fallen victim to this." Balancing between allocating time for the 'doing' and for discussion and reflection is a constant dilemma for these nonprofits. 
While some nonprofits are more successful than others, "keeping everyone on the ship" is an ongoing challenge. A number of the nonprofits, for example, have language barriers among staff that inhibit communication, with staff members whose native language is either Hebrew or Arabic or English. In one organization, all staff meetings are held in English because it is the most common language; however there are staff members who are clearly struggling to communicate in English. ${ }^{38}$ It is a problematic issue without an easy solution, especially because staff meetings, together with informal interactions and collaborative work, are important to foster knowledge sharing. How these nonprofits, specifically the organizational leadership, are creating opportunities for collective inquiry to further knowledge production and exchange is explored next. The following two sections, focusing on the role of organizational leadership in nurturing a learning environment, provide more insight into factors that can inhibit or facilitate social capital.

\subsubsection{Key Facilitator for Social Capital: Experience/ Origin of Leadership}

Social capital, which can help to provide a sense of stability and connection among staff to an organization, is especially significant for nonprofits in the sample. Their work is quite difficult, often unpredictable, and the road can be rather bumpy; as one staff member of an Arab women's organization cited the expression in Arabic, "one day is honey, one day is onion." The organizational leadership plays a vitally important role in nurturing the level of trust among staff and providing support for professional and personal fulfillment. Both the value-based organizational structure that is embraced by most of the organization's leadership as well as the fact that so many of the current staff are original founders help to enhance the nonprofits' level of social capital.

A senior staff member who works with humanistic education reflected on her organization:

I believe that in order to continue working in a particular place there is a need to be creative, to always be developing new things and to find an opportunity for selfexpression at work. Innovation gives us the desire to work... Here we are careful to support each other all the time and allow individual staff members to make a contribution. We have all built this together gradually over time. We can't give bonuses or rewards, but we can give the staff a sense of how their work is valued.

\footnotetext{
${ }^{38}$ This is based on observations of the researcher who was present for three staff meetings.
} 
This type of encouragement that is described above positively impacts on the level of morale among the staff, while also enhancing organizational learning. When there is a sense of openness and trust, the staff feel valued and believe that they are making a meaningful contribution to the organization (Davenport \& Prusak, 2000). This sense of valuing the individual contribution of staff members is critical for facilitating social capital. It contributes to sense of "coherence", where staff members feel that they are part of process of setting organizational strategies and have a confidence that decisions are made based on the knowledge that exists in the organization (Davenport \& Prusak, 2000).

At a nonprofit for Arab youth, the staff indicated a strong sense of trust in the leadership of the organization and enjoy being part of a very open environment. As the founder and director reflected on it, "we have an informal atmosphere, without real hierarchy; we are not a big organization but a small team. There is lots of room for imagination and initiative." Another staff member explained, "After all of our growth certain things have not changed. The director stays as modest as he has always been. There is still a great deal of openness. And each of our contributions is valued."

This 'modesty; of the leadership is of significance in nurturing social capital. "Openness and trust are tightly coupled" (Cohen \& Prusak, 2001, p. 47). In the majority of organizations the leadership operates with a high level of transparency. For example, the organization's transparency is reflected in its physical transparency (see Subsection 2.1.2.6); in seven out of the ten organizations, the director or veteran staff members share open office space with other staff members. Both the open physical access, as well as open access to information, helps to nurture trust; every staff member can feel that they know "what's going on" and can contribute to and access the knowledge base of the organization.

Cohen and Prusak (2001) cite longevity as a social capital indicator in organizations: length of tenure at an organization can indicate that a staff member has a level of identification with the organizational values, a sense of loyalty and a close connection with other members of the organization. ${ }^{39}$ As an indicator of social capital, longevity also contributes to the social capital in an organization because "relationships and trust develop over time" (Cohen \& Prusak, 2001, p.

\footnotetext{
39 This is not to minimize the benefits of 'new blood' in an organization. Among several nonprofits in the sample, new staff members have provided the organization with an 'outsider's' perspective, which is of great value.
} 
141). ${ }^{40}$ Among the nonprofits in the sample, the bulk of the leadership and staff have stayed with the organization since its official establishment (the average life span of the nonprofits in the sample is six years, not including the years prior to its official registration as a nonprofit). For example, the director of a nonprofit who works with Ethiopian families was part of an informal group of volunteers for many years. As an immigrant from Ethiopia, she and other volunteers spent over five years on an informal basis helping newly arrived families from Ethiopia. Only after the most recent wave of Ethiopian immigrants in the late 1990's did the seed group decide to formalize their activities and establish an organization. A staff member talked about the director:

She has demonstrated such a long term commitment to the cause; she volunteered for so many years simply because she wanted to help these families. What we do here is essential and can make all the difference in the integration of Ethiopian children into Israeli society...We are all invited to take part in what has been created. We have great respect for her and she also respects our input.

In the few nonprofits that have experienced significant turnover, the leadership has been drawn from the 'rank and file'. At one of the nonprofits, for example, the current director is a former social worker. As a staff member explained, "She does not come from 'management' but is one of us and she understands us. To her, the numbers are not what matters, but the 'how'- how the goals are achieved." In part because the director is viewed as "one of us", she is able to nurture trust by allowing the staff to feel supported and heard. Another staff member commented on her leadership,

The director does not give orders without explanation; she is not telling you what to do but asking you what you think. We feel a respect for our professional experience and our ideas. This of course impacts on our motivation and ability to give.

${ }^{40}$ Cohen and Prusak (2001) also note that low turnover, in addition to being an indication of a higher level of commitment to the organization, also helps the organization by saving resources on recruitment training, maintaining organizational knowledge and ensuring continuity and cohesion among the staff. 
It is important to remember, however, that these organizational strengths can also be their weaknesses (see Subsection 2.1.2.4). Sometimes the collegial atmosphere characterizing many nonprofits in the sample has led to problem of 'authority', as is the case in the nonprofit cited above. The director explained,

I come from the staff, the same, equal background. I work to make it an environment where everyone feels a part of the decision making process. But there is an art to balancing the open conversation with the placing of borders. Some staff can use democracy in not a fair way, to express their anger. This is especially problematic if they do not have the organization's interest at heart. It can sometimes be destructive. I am now working on how to set borders.

This director is currently working with a capacity building consultant on developing her leadership and management style. Another director in the sample has recently completed a leadership training course offered to nonprofit managers and is grappling with similar issues. The leadership of these nonprofits, generally successful in creating very open work environments that are characterized by cooperation, are sometimes challenged to find the balance between acting as a 'colleague' versus a 'director'. A very different type of organizational leadership problem is addressed in the next section.

\subsubsection{Key Barrier to Social Capital: Unaligned Organizational Change}

What happens when there is a lack of organizational policies that nurture trust and efforts to address the problem are not necessarily genuine? In light of how many of the nonprofits in the sample are engaged in efforts to strengthen their capacity, it is an important question to consider. Without the 'readiness' to change, any efforts to enhance effectiveness or performance will encounter strong resistance (see Section 2.4.6).

In one of the organizations, for example, there are a variety of issues that are negatively impacting on the morale of the staff and ultimately affecting overall performance. As one staff member confided: "We are run more like a family than an organization... This makes the environment one of who is 'daddy's favorites' and wanting to please 'dad'. 'Sibling rivalries' ensue." Another staff member described the work environment: "this is not an atmosphere where constructive dialogue can take place. Here it is more like table tennis than dialogue." In an 
organizational retreat that was designed to enhance organizational impact, the problematic work environment unexpectedly became the central focus.

The developments since the retreat illustrate the potential hazards of beginning to engage in some sort of capacity building process that is not holistic or genuinely pushed by the leadership (see Blumenthal, 2004). A long list of measures was identified after the retreat in an effort to address the problems raised by staff members. For example, to encourage more professional interactions among staff, organizational norms were established that included a list of core principles such as respect, compassion, accountability and dignity. The organizational structure was changed on paper in an effort to presumably disperse authority. The organizational leadership promised, among other things, to help clarify job descriptions, standardize both fringe benefits (which were perceived by staff as randomly awarded) and contracts (the lack of contracts were seen as being used to "control" staff).

However, as the organization underwent reorganization, it was unclear as to what were the main forces driving the change and to what extent the staff expectations were managed regarding how fast organizational change was actually possible (see Section 2.2.2.1). Six months after the retreat took place, many members of the staff indicated that their faith in the organization was lower than ever. With the exception of adjustments in the organizational structure, there was no follow up on any of the promised actions on behalf of the leadership. Recommendations such as adherence to the newly defined organizational norms and 'core principles' of respect or dignity had turned into a source of jokes. As a staff member commented, "Maybe we have a desire to be horizontal, but we are vertical. The new organizational structure is not reflective of the current decision making structure." According to another staff member, "The status quo remains and there is no real change. It is only in words that there is any encouragement towards the sharing of information and power." Many of the staff talked about the gap between theory and practice. A frustrated staff member who is considering leaving the organization described his perception of the situation:

The director feels a challenge to the status quo is a challenge to him and there is a threatening, abusive dynamic when mistakes are made. The staff is afraid to do anything wrong because he is like a warden, a bully, and he remains as defensive and evasive as ever. 
According to Cohen and Prusak (2001), trustworthiness, a key aspect of social capital, "can only be demonstrated, not asserted" (p. 45). The reward system in an organization signals to staff members what type of behavior is valued (Cohen and Prusak, 2001). In the example cited above, there is an understanding that the organizational leadership indicates that loyalty to the director takes precedence over the open exchange of ideas. After the organizational leadership did not follow through on promised changes, the faith of many staff members, already wary of existing organizational policies that do not nurture their trust, plummeted even further.

Light (2004) writes that "organizations can be very effective in achieving their mission and not be well managed, meaning that organizational effectiveness is not necessary for high performance.... Yet poorly run organizations cannot produce program impacts for long” (p. 21). While the organization cited above may continue to make a social impact, it is questionable as to how long it will be able to sustain itself in the long term. Especially in the challenging work environments that tends to characterize these nonprofits, it is essential for staff to feel valued, respected and to have a sense of trust in the organization. A staff member of a different organization considered the issue of the staff's personal and professional fulfillment:

I come from the private sector and I see that the nonprofit world can be chaotic at times. People are working for a living. They have ideology and passions but they are also making a living. There is a fine line. Borders get faded and unclear. The more mechanisms that draw lines between work and private are good. We are a resource that makes the organization work - it matters whether we are unhappy, burnt out or frustrated. Everyone is replaceable, but an organization can function better if these issues are taken into consideration.

In the past year, this organization established a human resources committee made of up volunteers and board members who act as 'mentors' to the staff. It was determined that the 'mentors' could play an important role in helping to preserve the non-hierarchical organizational structure. Each staff member chooses their 'mentor' who can help them with personal and professional development. The staff members meet individually with their mentor once a month, and the mentors, who together comprise the human resources committee, also meet as a group once a month. According to interviews with staff and board of the organization, the fact that the human resources committee includes board members helps to ensure continuity, a commitment to the process, and ensures that the problems or ideas that are raised are also addressed by the board. A staff member reflected on the importance of the human resources committee: 
The committee, in addition to the board, helps to monitor the organization. The crisis that we had in the past year occurred because there was no monitoring of the organization or the relationships between staff members. We now receive feedback that we did not have before and the whole process helps to provide us with stability. In the private sector, there is a human resources person ... you have an address. In many nonprofit organizations, this does not exist. The new committee that is set up now gives us a person to take care of your needs and express to the decision makers of the organization, a 'representative' for you.

One of the keys to enhancing performance through capacity building is to "pick the right answer for the right problem at the right time and for the right reason" (Light, 2004, p. 134). When a nonprofit understands its organizational culture and carefully determines the needs and the type of intervention that is required, the chances of success in capacity building will be much greater. Moreover, the capacity building efforts will serve to strengthen rather than harm the existing social capital in the organization. Results of the research concerning the fifth and last dimension of adaptive capacity, the external connectedness of a nonprofit, are discussed in the following section.

\subsection{NETWORK/EXTERNAL CONNECTEDNESS}

Nonprofits with adaptive capacity are very aware of what is occurring outside the boundaries of their organizations. As defined by Sussman (2004), a nonprofit with network connectedness is intent on interacting with its external environment and seeking outside perspectives. There is an understanding that it is possible to more successfully fulfill the organization's mission by developing strategic alliances and interdependent relationships with other organizations. ${ }^{41}$ Nonprofits, including those in the study sample, need to constantly resist a "natural tendency to become organizationally introverted by incessantly pushing the organization to be outwardly directed" (Sussman, 2004, p. 2).

Nonprofits should proactively take steps to ensure that they do not become isolated from their surrounding environment. This is even more of a challenge because "nonprofits often create an unstated but real environment of rivalry and contention among themselves" (McHargue, 2003, p.

\footnotetext{
${ }^{41}$ External connectedness, as defined by (Sussman, 2004) includes the ability of an organization to elicit feedback from outside the organization, an important aspect of evaluative thinking that was discussed in Section 4.3. This discussion focuses primarily on a major aspect of external connectedness, the nature of cooperation with other organizations.
} 
197). Among the nonprofits in the sample, it is difficult to gauge the extent to which they are able to overcome this 'rivalry'. During the interviews, staff members emphasized the importance of their cooperation with other organizations. It is readily apparent that the staff place value on the relationships that have been developed with a variety of institutions, ranging from local universities, ${ }^{42}$ to neighborhood community centers. Several of the nonprofits benefit from being part of umbrella organizations that offer a variety of support. For example, the nonprofit promoting the rights of Arab women, a 'spin-off' of a larger women's organization, shares office space with other feminist nonprofits and is a member of a network of like-minded organizations. Similarly, the nonprofit working on environmental issues shares office space with other environmental organizations and enjoys the benefits of being able to readily exchange information. The personal connections that develop in such circumstances can enhance organizational learning, especially if 'communities of practice' are formed. ${ }^{43}$ Indeed, the intimate nature of the social change movement in Israel is conducive to the development of networks based on the personal as well as professional relationship between staff members (Asa, 2005). The 'networks' of the nonprofits in the sample extend outside of Israel. Several staff members discussed the use of the internet in connecting them to the staff of other nonprofits who are operating in similar fields and the advantages to their participation in an international knowledge network. $^{44}$

Especially for smaller nonprofits, of which the sample is comprised, it is possible to increase their potential for impact through relationships with other organizations. These types of relationships among nonprofits can lead to 'system-level effects' that can help to promote their mission (Sussman, 2004, p. 3). During the interviews, staff members at a variety of the nonprofits emphasized the importance of cooperation in advancing the overall social change movement. For example, one staff member discussed how cooperation is important in helping to maximize his nonprofit's impact, explaining that "cooperation plays a vital role in building democracy. It is

\footnotetext{
42 Many of the organizations, for example, are hosting college students who are carrying out research projects or fulfilling their intern requirements at the nonprofits.

${ }^{43}$ See Subection 2.1.2.4 for more information about 'communities of practice'.

44 The use of the internet was cited as critically important by several organizations. Staff at the organization working with the Russian-speaking community spoke about the impact of the civil society movement that blossomed in Russia in the late 1990s, and the extent to which organizations in Israel learned from their colleagues abroad. Staff at the Arab youth organization also discussed how the internet has enabled them, for the first time, to be in such close contact with their counterparts outside of Israel.
} 
especially critical among social change organizations that should be a model for society." Another staff member at a different organization talked about the significance of cooperation in helping to develop the field and the entire nonprofit sector as a whole; she concluded, "that is the way to social change, and the only way is if we work together."

In the questionnaire, staff members were asked to answer items relating to issues such as networks, partnerships and cooperation with other organizations. Staff overall indicated that their organizations are outwardly directed in the sense that they are somehow connected to their external environment. For example, the majority of the organizations scored relatively high in questions such as "It is worthwhile to work with other organizations because it allows us to achieve more that we could individually as an organization" and "We actively participate in a network of like-minded organizations in order to exchange knowledge." Respondents were asked on a scale of 1-5 (1: strongly disagree and 5: strongly agree) to rate the extent that they agree with the above statements. The organizational mean for the item: "It is worthwhile to work with other organizations because it allows us to achieve more that we could individually as an organization" is 4.1, while the organizational mean for the item: "We actively participate in a network of likeminded organizations in order to exchange knowledge" is 4.0.

In an open-ended item in the questionnaire, staff members were asked to complete one of the following sentences: "We proactively cooperate with other like-minded nonprofits organizations because..." or "We would proactively cooperate with other like-minded nonprofits organizations more frequently if..." A sample of the responses to the sentence, "We proactively cooperate with other like-minded nonprofits organizations because..." includes: ${ }^{45}$

- "It helps us to check ourselves and receive feedback and learn from other organizations."

- "It adds knowledge, information, and strength."

- "It is part of our ideology and strategy."

- "We partner because, to us, the mix of resources and efforts that are directed to the same goal can advance the specific issue much more effectively than the organization itself."

- "To increase our impact."

- "We believe in the exchange of experience, etc. that occurs during this kind of cooperation. We can't all do this work alone."

- "We believe that there is always something to learn, on one hand, and on the other hand, we have expertise that we are happy to share with other organizations."

${ }^{45}$ Out of the 26 responses to this item, 10 respondents chose to complete the sentences: "We would proactively cooperate with other like-minded nonprofits organizations more frequently if...". Sixteen respondents chose to complete the sentence: "We proactively cooperate with other like-minded nonprofits organizations because...". 
A sample of the responses from those who chose to complete the sentence "We would proactively cooperate with other like-minded nonprofits organizations more frequently if..." are presented below:

- "If we saw that it was effective. The majority of time it is lots of time and little practical outcomes."

- "If it was more effective for our fundraising efforts."

- "If there was more time, and put it as a top priority."

- "If we had more money and more resources."

- "If we thought that is our central goal of the organization."

- "If we thought it was effective to further our goals."

- "We need openness among the staff and go down from our ego that we are the best."

Certainly there are many challenges, as cited above, to the nurturing of cooperation between likeminded organizations. For example, nonprofits may perceive the allocation of scarce resources to the creation of networks as a potential 'risk'. As a board member of one of the organizations explained:

Everyone is afraid of cooperation with other organizations because it requires a lot of energy until you can actually see the benefit. Our board needs to consider this issue more. There is not enough thought about what could be the benefits and how it could strengthen the organization and enable it to work better. Right now it is only seen as a risk that may not pay off.

It is possible to distinguish between the type of cooperation that exists with local community organizations versus nonprofits that considered as 'direct competitors'. ${ }^{46}$ The model of an independent nonprofit (unaffiliated with the government, a political party or a religious movement) is relatively new in Israel. Especially among certain communities, old perceptions may hamper efforts towards cooperation. A staff member of an organization that works with the Russian speaking community explained,

We have the ability to choose who we work with; we are independent and we are not connected to the government or political party. But there is a fear among other Russian speaking organization that was brought from Russia. It is the fear that if an organization is successful, it will 'eat' the others instead of helping them. It is based on the dictator model- that someone will take their power and try to take advantage of them. We have tried to convince other organizations that working together would make it easier for us to raise funds, receive publicity, etc. but this mindset persists.

\footnotetext{
${ }^{46}$ Within the sample of ten organizations, there are two nonprofits that are now directly in competition following a recent decision of one of the organizations to expand its scope.
} 
While cooperation may not always be possible, it is critical that organizations are somehow engaged with their external environment. Yet one of the problems cited by a capacity building consultant is that nonprofits are not necessarily carrying out needs assessments of their community and determining how they will distinguish themselves from other nonprofits (Asa, 2005). In the questionnaire, the responses to an item relating to this issue only indicated further ambivalence on this issue. The organizational mean for the item "When we review our programs and policies, we routinely consider what other organizations in our field are doing" was only 3.4. Understanding one's niche and how the organization "fits within its local social ecosystem" is a key factor in ensuring sustainability (David, 2002, p. 4). Operating in an environment that is constantly changing, learning organizations understand that, "no niche is permanently secure" (David, 2002, p. 4). The following two sections discuss a key facilitator and a key barrier to 'network connectedness'.

\subsubsection{Key Facilitator to Network Connectedness: Interdependent Nature of Programs}

For many of the nonprofits in the sample, cooperation with other organizations is a key part of their strategy for project implementation. One aspect of adaptability is the ability to turn a constraint (lack of resources or funds) into a positive. Either out of necessity or ideology, these nonprofits benefit from the partnerships that they nurture with other organizations.

For those organizations that operate on a national level, partnerships with local community organizations are particularly important. The Arab youth organization, for example, has a small headquarters office, and coordinates over twenty youth groups operating around the country. All the workshops, events and activities of the youth groups are carried out though partnerships with other organizations. The director explained,

We hope as much as possible to cooperate with other organizations. It is good for our strategy and also it makes sense practically because we cannot fund all of our activities. We only own this one building, so we work with existing organizations (community centers, schools, etc.) and invite other organizations to take part in our activities and take part in our goal for building youth leadership. We can all benefit. It is not always smooth but it usually works. 
Another example of a nonprofit in the sample that works nationally is the organization that promotes economic sufficiency for women. A staff member emphasized the importance of cooperation as a fundamental component of their strategy:

\begin{abstract}
We cannot implement any of the activities without cooperating with other organizations. We create a coalition of organizations, such as the immigrant absorption office, the municipality's social welfare department, and other community nonprofits, and they are the primary local recruiter and organize it locally. Our local partners are crucial to the success of the program. On principle, we do not do it alone but always build our projects with local partners. We see it as a goal that all of our projects have partners. There is an ideology behind it and an awareness of the importance and advantages of this cooperation. Feminist ideology is built on cooperation, and there is not a lot of ego of the organization. We focus on the goal.
\end{abstract}

As indicated in the interviews as well as questionnaire responses, the ideology of cooperation is a strong motivating force driving efforts to connect with other organizations. While there may be a natural competition that exists or a "tendency to become organizationally introverted" (see above), many of the organizations in the sample are seeking to ensure that they construct interdependent relationships with other organizations. It is important to consider however, the degree to which the nonprofits are staying linked to their community and target population. One aspect of this ongoing challenge is addressed in the following section.

\title{
4.5.2 Barrier to Network Connectedness: Funding Disconnect from Local Community
}

Nonprofits with adaptive capacity are intentional about interacting with their external environment, understanding that this interaction "provides information-rich feedback, stimulates learning and ultimately prompts improved performance" (Sussman, 2004, p. 3). Any nonprofit, however, still has to overcome the fact that, in general, there is not a direct connection between the 'output' or 'product' of the nonprofits and the resources that fund the 'output' (McHargue, 2003). For example, a nonprofit in the sample, which is involved in advocacy efforts to promote environmental-friendly policies and protect open spaces in local neighborhoods, receives the bulk of its funding from several foundations in the United States. In theory, the 'customers' (i.e., the local residents) would 'buy' the product offered by the nonprofit. In contrast to the private sector, the type of situation that is common among nonprofits is problematic because, as McHargue 
(2003) explains, "this disconnect between funding and outcome can convolute accountability, performance and mission" (p. 197). While nonprofits are often forced to overcome this 'disconnect', it can be especially challenging when the nonprofits receive their funds from abroad as tends to be the case with the nonprofits in the sample.

The social change movement in Israel is primarily funded by international foundations and donors. According to an Israeli fundraising consultant, about $80 \%$ to $90 \%$ of the funds are raised abroad (Asa, 2005). There is growing awareness and encouragement among nonprofits to carry out activities such as membership drives and community fundraising events, but this type of fundraising is relatively new to Israel (Asa, 2005). ${ }^{47} \mathrm{~A}$ fundraiser in one of the nonprofits in the sample considered her organization's strategy:

We are facing a serious challenge at this stage of the organization. We have reached the five year point; the foundations that have funded us are now finishing their support and the grants are at their conclusion. It is now an imperative that we consider how to change our strategy. It is no longer feasible for us that $85 \%$ of our funding is from North America. Currently we are working on a variety of efforts to fundraise in Israel, both among Israeli companies and local individuals.

Similarly, a fundraiser at another nonprofit discussed their changing approach to fundraising,

It was considered revolutionary only a few years ago to hold a local fundraising event in our community. We have to begin to work in this direction. Corporate giving is growing all the time. And the mindset towards organized giving is changing among Israelis. They have always been very generous; we just need to learn how to organize the efforts better and be more creative.

While there is a growing trend towards local, community-based fundraising, the bulk of the nonprofits resources still come from foundations or individuals outside of Israel. Asa ( 2005) questions the impact on the development of these nonprofits, a process that can be distinguished perhaps from many nonprofits operating in the United States. The social change nonprofits in Israel, rather than starting out as locally funded, establish themselves by receiving funds from abroad and perhaps only at a later stage begin to connect to their local community for fundraising. On the whole, the nonprofits in the sample are not carrying out 'grassroots' or community-based

\footnotetext{
${ }^{47}$ The development of social change nonprofits is coinciding with the downsizing of a traditionally strong social welfare state (which is accompanied by a very high tax rate) (See Doron, 1985). In recent years, philanthropic efforts have become more organized and modeled on traditional fundraising activities of nonprofits in the United States.
} 
fundraising. As such, their accountability to the community is different than it would be if the local funders were involved in the operations of the nonprofit. Moreover, there is little expectation placed on the board to be involved in fundraising and the 'long distance' funders do not necessarily have a familiarity with the local landscape or an awareness of the community dynamics. It is absolutely essential, therefore, for these organizations to proactively be in dialogue with their external environment and continually assessing their 'niche'. The degree of 'external connectedness' among the nonprofits, as well as the other four attributes of adaptive capacity, is summarized in the following section. 


\subsection{SUMMARY TABLE ON ASPECTS OF ADAPTIVE CAPACITY}

The research findings are summarized below in Table 13. Identifiable patterns among the ten nonprofit organizations are addressed as well as selected key facilitators and barriers. In the next and final chapter, Chapter Five, the conclusions and implications of the study are discussed.

Table 13 : Aspects of Adaptive Capacity for the Participating Nonprofits

\begin{tabular}{|c|c|c|c|}
\hline & Identifiable Patterns & Key Facilitators & Key Barriers \\
\hline Shared Vision & $\begin{array}{l}\text { - Strong on consensus of } \\
\text { mission, difficulties in } \\
\text { coping with the creation of a } \\
\text { cohesive strategy }\end{array}$ & $\begin{array}{l}\text { - Integration of } \\
\text { Organizational } \\
\text { Vision with } \\
\text { Personal Vision }\end{array}$ & $\begin{array}{l}\text { - Unclear role of } \\
\text { Board }\end{array}$ \\
\hline Openness & $\begin{array}{l}\text { - Characterized by } \\
\text { collaborative work } \\
\text { environments that allow for } \\
\text { diversity of opinion, } \\
\text { encountering challenges in } \\
\text { articulating a learning } \\
\text { agenda }\end{array}$ & $\begin{array}{l}\text { - Value-Based } \\
\text { Organization } \\
\text { Culture }\end{array}$ & $\begin{array}{l}\text { - Maintaining } \\
\text { 'organic roots' } \\
\text { in the midst of } \\
\text { growth }\end{array}$ \\
\hline $\begin{array}{l}\text { Evaluative } \\
\text { Thinking }\end{array}$ & $\begin{array}{l}\text { - Mixed on how much } \\
\text { evaluation is 'mainstreamed' } \\
\text { into the organization, } \\
\text { question of the extent to } \\
\text { which evaluation is } \\
\text { integrated into planning and } \\
\text { if there is a clear evaluative } \\
\text { strategy }\end{array}$ & - Desire for Impact & $\begin{array}{l}\text { - Gap between } \\
\text { organizational } \\
\text { culture and } \\
\text { performance } \\
\text { culture }\end{array}$ \\
\hline Social Capital & $\begin{array}{l}\text { Enjoy social networks and } \\
\text { supportive work } \\
\text { environments, challenged to } \\
\text { continually provide } \\
\text { frameworks for knowledge } \\
\text { exchange }\end{array}$ & $\begin{array}{l}\text { - Experience/ } \\
\text { Background of } \\
\text { Leadership }\end{array}$ & $\begin{array}{l}\text { - Unaligned } \\
\text { organizational } \\
\text { change }\end{array}$ \\
\hline $\begin{array}{l}\text { External/ } \\
\text { Network } \\
\text { Connectedness }\end{array}$ & $\begin{array}{l}\text { - Connection to outside } \\
\text { community but questionable } \\
\text { cooperation with other, like- } \\
\text { minded nonprofits. }\end{array}$ & $\begin{array}{l}\text { - Interdependent } \\
\text { Nature of Programs }\end{array}$ & $\begin{array}{l}\text { - Funding } \\
\text { disconnect } \\
\text { from local } \\
\text { community }\end{array}$ \\
\hline
\end{tabular}




\subsection{CONCLUSION AND IMPLICATIONS}

This final chapter begins by a brief review of the purpose and scope of the study. A summary of the findings is then presented, followed by a discussion on directions for future research. Lastly, the chapter concludes by exploring the implications of the research findings.

\subsection{REVIEW AND REFLECTIONS ON THE STUDY}

In an environment that is characterized by both growing needs and shrinking resources, nonprofits around the world are challenged to effectively confront complex social issues and create social value. Their sustainability depends on their ability to change and adapt to varying circumstance; indeed this 'adaptive capacity 'is considered as an essential organizational capacity for nonprofits (Letts, Ryan \& Grossman, 1999). It is adaptive capacity that supports learning, innovativeness and responsiveness (Letts, Ryan \& Grossman, 1999; Sussman, 2003). Yet research findings suggest that not only is adaptive capacity one of the weakest components of organizational capacity among nonprofits, but that capacity building efforts are not necessarily focusing on strengthening this key dimension of organizational effectiveness (see Connolly \& York, 2003).

Due to the socio-political nature of Israel, and in light of the violence in recent years, Israeli social change nonprofits are especially required to display a high level of adaptive capacity. Representing the backbone of Israel's civil society, their ability to adapt and sustain themselves is critical to strengthening the country's democracy. Therefore, to learn more about how these nonprofits create a 'generalized coping mechanism' to deal with change and unpredictability, this study developed and tested a theoretical framework for the assessment of organizational readiness to engage in the ongoing process of adaptive capacity building. This theoretical framework was subsequently applied to ten social change nonprofits in Israel. 
Through the use of a questionnaire and interviews, the study sought to gain insight into the work environments and learning cultures of the organizations.

In reflecting upon the study, the research findings emphasized the extent to which, as noted earlier in Chapter Two, the categorization of the theoretical framework is overlapping in its nature. The 'boundaries' between the aspects of adaptive capacity are somewhat artificial, as each of the five dimensions are mutually reinforcing. While keeping this in mind, the categorization did help to provide a focal point for exploring the issues. It also later facilitated in the analysis of the data. In regards to the methods of the study, the questionnaire was originally developed to be used in conjunction with interviews. As the research got underway, it became clear that the questionnaire can indeed help to provide an overall 'picture' of each organization, but it was of critical importance to interview as many staff members as possible. Ultimately, the richest research findings came from the qualitative interview data. A summary of the findings is provided below.

\subsection{SUMMARY OF THE RESEARCH}

By exploring the perspectives of the staff and board members, the study aimed to understand the organizational culture and the day-to-day realities of organizational life that help to shape the adaptive capacity of the nonprofits in the sample. Overall, the organizations were given rather high marks by staff and board members on four of the five adaptive capacity dimensions, with evaluation of performance as one of the main exceptions. The following discussion summarizes some of the major findings drawn from the data by addressing several specific facilitators and barriers that affect a nonprofit's ability to adapt continually to its environment.

\section{Key Facilitators to Adaptive Capacity}

In general, the staff members of the nonprofits exhibit a strong commitment to their organization's mission and a deep sense of pride in their work. The organizations in the sample tend to be characterized by high levels of social capital and enjoy an open, collaborative work environment. A 'spirit' among these nonprofits, which infuses staff members with an energy and a sense of 
purpose, is an important element contributing both to adaptive capacity and, ultimately, to the sustainability of the organizations (David, 2000). Operating in the highly volatile Israeli socialpolitical context, the staff of these nonprofits remain remarkably motivated and committed to their organizational vision. This dedication to the organizational vision among staff members is coupled with a keen appreciation for the urgency of their work.

Among the ten nonprofits, an integration of the organizational mission with the personal goals of the staff is an important factor in strengthening the overall shared vision, a key component of adaptive capacity. These nonprofits enjoy an important attribute of learning organizations: the intertwining of personal fulfillment and professional fulfillment of the staff. In general, the staff members of the nonprofits in the sample tend to view themselves as partners in building the organizational vision and take pride in the open, informal spirit that often characterizes their work environment. As many of these organizations continue to grow, the full engagement of the staff should serve them well in the ongoing challenge of creating full alignment between the organization's vision, strategy and capacities.

Tackling challenging social problems under typically stressful working conditions, the staff of most of the nonprofits benefit from high levels of social capital, another major dimension of adaptive capacity. The majority of these nonprofits, many of whose stated goal is to create a more just and democratic society, are conscious of linking their organizational values to their organizational management style. Moreover, the organizational leadership tend to operate with a high level of transparency. Both the open physical access in the nonprofits, along with the open access to information, allows staff members to feel that they know "what's going on" and helps to nurture trust. Staff members generally report feeling comfortable asking questions, raising difficult issues and reflecting on mistakes. As such, the nonprofits are therefore better able to tap into the collective nature of knowledge; staff members feel comfortable exchanging information and working together cooperatively. While enhancing organizational learning, their work environments contribute to the generally high level of morale that is maintained among the staff under considerably difficult circumstances. Staff members tend to feel valued for their skills and experience, and have the sense that they are making a meaningful contribution to their respective organizations. This serves to facilitate and reinforce the existing social capital, as staff members have confidence that decisions are made based on the knowledge that exists in the organization. 
The nonprofits in the sample also typically benefit from a degree of external connectedness, another important dimension of adaptive capacity. While it is difficult to gauge the extent to which these nonprofits are able to overcome the inherent competition between them, it is readily apparent that many of the staff members place value on the relationships and partnerships that have been developed with a variety of institutions. For smaller nonprofits in particular that made up the sample, cooperation with other organizations is an essential part of their strategy for project implementation and allows them to increase their overall system-wide impact. Indeed, staff members often emphasize the importance of inter-organizational cooperation in terms of advancing the overall social change movement. The ideology of cooperation is a strong motivating force driving efforts towards cooperation.

All of the organizations, operating for at least five years, have exhibited some type of adaptive capacity in their ability to navigate the dynamic, often turbulent Israeli environment. While seeking to create a presence, these nonprofits generally indicate a strong desire to be constantly improving and finding ways to enhance their social value. As a staff member from a nonprofit in the sample comments, "We are $85 \%$ established. We want to keep $15 \%$ of us always unestablished." This comment is reflective of how most of the organizations view themselves working to create a presence in their environment while constantly developing and growing. Yet many of the nonprofits, as they move into the 'enterprising phase', are encountering difficulty in their reluctance to leave the 'organic phase' behind (see Subsection 4.2.2). The discussion now turns to certain challenges that the nonprofits are facing in enhancing their adaptive capacity.

\section{Key Barriers to Adaptive Capacity}

As many of the nonprofits in the sample move up the spiral of development (see Light, 2004), the intimate and informal nature that characterized the establishment phase of nonprofits in the sample is slowly being transformed. With a strong commitment and few resources, the founders of these nonprofits generally started by identifying a societal need that is not being met and, in an admirable way, jumped right into the 'doing'. Key decisions were not necessarily made about how, for example, to track impacts or establish reporting routines. The majority of these nonprofits are now finding that as they move into a new phase of development, it is essential to have very clear systems in place for learning and evaluating performance. It is an ongoing challenge that organizations are struggling to cope with effectively. 
Among the nonprofits in the sample, there tends to be divergent views, more so than with other aspects of adaptive capacity, regarding the use of evaluation to plan, measure progress, and assess community needs. Taking on difficult social problems where progress is both hard to make and challenging to measure, the nonprofits often encounter difficulty in defining and measuring the outcomes of their work. To further complicate matters, there tends to be a variety of interpretations among the staff and board regarding the purpose, the process and the use of evaluative results. Certain staff members see evaluation as an activity that supports organizational learning, yet others primarily view it as something that is driven by external demands for accountability. Even among those nonprofits that have a clear understanding of their evaluation strategy, staff members face numerous challenges, such as lack of time, resources and experience, in developing and executing an evaluation plan. Funded primarily from abroad, these nonprofits are also coping with the disconnect between the 'output' of the nonprofits and the resources that fund the 'output'. It is therefore essential for them to be proactively in dialogue with their external environment and continually assessing their 'niche,' or they may someday find themselves entirely irrelevant in their current context.

A learning 'agenda', which accompanies an evaluation strategy, is another key element in promoting adaptive capacity. Yet, whereas some of the nonprofits have a well-conceived learning strategy, many in the sample appear to be struggling with managing the knowledge of the organization. Especially in the face of growth, it is increasingly difficult for these nonprofits to provide time and space for their staff to learn together in order to continuously improve organizational performance. Many of these nonprofits are now increasingly challenged, especially with so many part time staffers, to ensure that knowledge becomes 'institutionalized' and continually 'moves' through the organization. Operating in multi-cultural settings and with few resources to invest in skill development, these nonprofits have to be especially strategic in developing a learning agenda and in articulating their knowledge needs.

A well-functioning nonprofit board has the ability to take an organization temporarily out of 'crisis mode', guiding it to focus on ways to improve overall adaptive capacity (see Gray, 1998). Indeed, the boards of the nonprofits in the sample could play a particularly important part in further advancing the use of evaluation. The boards, however, tend to have an undefined role in helping the organizations to articulate what knowledge is needed to successfully pursue the 
organization's strategic goals and to systematically evaluate their work. The ambivalence that often characterizes the board's role presents an important barrier to organizational learning.

As indicated in the research findings, the nonprofits are facing a variety of challenges that can potentially inhibit their ability to engage in adaptive capacity building. They are searching for the right mix between work environments that prize informality and openness with the need to establish systems and structures that can enhance learning and provide stability. A careful balance between various aspects of the organizational culture can ensure that organizational strengths do not paradoxically become weaknesses. For example, some of the leadership of these nonprofits, generally successful in creating open, cooperative work environments, have subsequently encountered difficulties asserting authority and ensuring staff accountability. Similarly, those organizations with high social capital also have to be careful to see if staff only reinforce each others' beliefs and inadvertently prevent the organization from seeking external input. A lack of understanding of how a nonprofit must continue to evolve and adapt to the demands of new realities will ultimately hamper its ability to maximize its social impact. These issues are especially timely in this particular national context, with so many of the Israeli social change nonprofits reaching similar stages of development.

\subsection{DIRECTIONS FOR FUTURE RESEARCH}

The field of nonprofit capacity building and nonprofit organizational learning is a relatively new area of study, and as such, there remain a large number of potential avenues for future research. Research is needed both to contribute to the overall understanding of adaptive capacity building, and to the development of methodologies to assess organizational readiness for capacity building efforts around the world. With specific regard to the context of the study, it is a particularly apt time to carry out this kind of research in Israel.

There are a various aspects to the present study that could be explored in greater depth, by either enlarging the sample or lengthening the time of the nonprofits under study. For example, a natural step would be to expand the population of nonprofits along key dimensions, (i.e., organizations with high growth or low growth; high rate of turnover or low rate of turnover). Or alternatively, it would be interesting to analyze several detailed case studies of a few 
organizations and explore how particular factors impact on their adaptive capacity over a period of time. Another possibility would be to enlarge the research sample to include 'unsuccessful' nonprofits that did not sustain themselves beyond five years. There are valuable insights that could be gained by learning from the experience of organizations that were unable to continue their operations past a certain period of time.

Further research will allow a greater understanding of how Israel's multi-cultural society and political context shape the adaptive capacity of local nonprofits. Indeed, many of the trends that are affecting nonprofits in Israel are also taking place in many countries around the world. A study, for example, that tracks how the changing landscape of local fundraising influences Israeli nonprofits could result in interesting comparisons with other national settings.

A great deal of research remains to be done in analyzing the link between organizational readiness to engage in capacity building and the success of capacity building efforts. The literature suggests that a more holistic approach to nonprofit capacity building and an increased focused on adaptive capacity would take place if consulting organizations invested more effort in assessing the nonprofit's organizational readiness to engage in change efforts (Connolly \& York, 2003). It would therefore be valuable to explore how an assessment of 'organizational readiness' can help to increase the likelihood that capacity building efforts are addressing the most pressing needs of the nonprofits. Moreover, with little research on the evaluation of capacity building efforts (see Blumenthal, 2003; Light, 2004), a study that analyzes their impact could make a meaningful contribution to the nonprofit literature. Drawing on Light (2004), a research study could examine perspectives of the nonprofits towards capacity building while, at the same time, tracking performance data. A related study would be to compare assessment data based on the perspectives of organizational stakeholders with performance data of the nonprofit over a period of time. Similar to the research carried out on for-profit organizations by Davidson (2001), the study could seek to find a determination of 'merit' for adaptive capacity.

Another direction for future applied research would be to accompany several nonprofits through an assessment of their adaptive capacity and then facilitate a reflection process with the organization. Undergoing the assessment process with nonprofits could engage organizational stakeholders, challenging them to ask questions and to understand the current condition of their organization. Indeed, in the present study, the data collection process often led the staff and board members to reflect on the meaning of adaptive capacity in their respective organizations. Due to 
the 'mismatch' that occurs between what consulting organizations provide in capacity building assistance and the actual needs of the organizations (Connolly \& York, 2003), it is especially important for nonprofits to gain an understanding of how to assess themselves and their needs. Furthermore, because the processes of improving organizational learning and developing organizational capacities are ongoing, the applied research could help nonprofit be equipped with the knowledge of how to carry out ongoing self- assessment and incorporate the learnings from the process.

Lastly, a study that analyzes the development of nonprofits and where they stand on a continuum of development in terms of adaptive capacity could contribute to the growing literature in the field of capacity building. Of particular interest would be an exploration of what types of capacity building strategies are of importance to nonprofits as they move up (and down) the 'spiral of sustainable excellence' (See Light, 2004). It would be of value to evaluate what is driving these efforts, as well as the organization's ability to continually adapt itself by both responding to and initiating change. As Light (2004) writes, "until the world stops changing and the future becomes certain, organizations can either change themselves or be changed by others." (p. 57).

\subsection{IMPLICATIONS FOR PRACTICE}

Theoretical implications can be drawn from the development of a conceptual framework for assessing the organizational readiness of nonprofits to engage in adaptive capacity building efforts. The findings from the study suggest that the five key dimensions of adaptive capacity represent a reasonable theoretical framework by which to analyze these issues. Moreover, this framework appears to be applicable for studying various national nonprofit contexts. The findings based on the application of the conceptual framework therefore seem to make a contribution to both the Israeli nonprofit literature as well as to the general nonprofit literature.

There are practical implications to the study as well, with special regard for the role of the funders, capacity building consultants, and nonprofit practitioners. As Light writes, highperforming nonprofits "invest in renewal even during the good times. They do not take success for granted or assume that their organization is working well just because they do not hear any 
complaints" (p. 145). How much the nonprofits in this study chose to invest in their capacity even when they did not "hear any complaints" is not entirely clear from existing data. While most nonprofits engage in capacity building efforts to address a specific problem, opportunity or crisis, (Light, 2004), funders can help organizations by providing resources and encouragement to carry out ongoing efforts to strengthen their overall organizational capacity. It is critically important to help these nonprofits strengthen their capacity while also respecting their organizational values and particular stages of development. It may, for example, be a disservice to push growth on organizations that do not find it suitable for them. ${ }^{1}$ With particular regard to evaluation, funders and consultants should consider how they can help to develop nonprofits' evaluative capacity, rather than simply imposing a framework for measuring program performance on those organizations that have few resources and little experience in the use of evaluation.

In terms of implications for the development of capacity building strategies, this study can shed light on possible focal points for enhancing adaptive capacity. In Table 14, which appears on the following page, a detailed list of potential pitfalls, accompanied by potential remedies, is outlined for each of the five dimensions of adaptive capacity. The potential pitfalls are based on the data from the study, while the potential remedies are drawn from the participating nonprofits, the nonprofit literature and the researchers' experience in the nonprofit sector.

\footnotetext{
${ }^{1}$ In Light's (2004) 'spiral of sustainable excellence,' the spiral upward was not based on growth but based on effectiveness and performance.
} 
Table 14: Potential Pitfalls and Potential Remedies for Enhancing Adaptive Capacity

\begin{tabular}{|c|c|c|}
\hline & Potential Pitfalls & Potential Remedies \\
\hline Shared Vision & $\begin{array}{l}\text { - Lack of consensus on the } \\
\text { implementation of the vision and } \\
\text { what are the specific goals to } \\
\text { achieve the organizational mission. } \\
\text { - Temptation to 'stray' from } \\
\text { organizational vision and widen the } \\
\text { core mission of the organization. } \\
\text { - Insufficient understanding among } \\
\text { staff of how their work relates to the } \\
\text { goals or mission of the organization. }\end{array}$ & $\begin{array}{l}\text { - Reinforce the existing commitment and motivation } \\
\text { by talking about the staff's connection to the vision. } \\
\text { Share up to date information with staff about the } \\
\text { decision making process regarding organizational } \\
\text { directions and engage staff in the process. } \\
\text { - Invest in the board and its understanding of strategic } \\
\text { planning. Make certain that board devotes time to the } \\
\text { discussion of vision and a cohesive organizational } \\
\text { strategy. } \\
\text { - Build consensus on how the organization will } \\
\text { implement its vision and ensure that there is } \\
\text { sufficient clarity of what are the organizational goals } \\
\text { and strategies. Invest time in mapping long -term } \\
\text { strategies and hold periodic planning sessions or } \\
\text { retreats with staff and board. } \\
\text { - Continually work on ensuring alignment between } \\
\text { vision, strategy and organizational abilities. Ensure } \\
\text { that the organization actively plans in anticipation of } \\
\text { how its external environment may change over the } \\
\text { next few years. }\end{array}$ \\
\hline $\begin{array}{l}\text { Innovativeness/ } \\
\text { Openness }\end{array}$ & $\begin{array}{l}\text { - Leaving the process of learning to } \\
\text { chance. Not consciously aware of } \\
\text { an organizational 'learning strategy' } \\
\text { that is essential for innovativeness. } \\
\text { - Insufficient investment in the } \\
\text { professional development of the } \\
\text { staff. Staff is not rewarded for } \\
\text { engaging in activities that may } \\
\text { enhance organizational learning. } \\
\text { - Imbalance between an } \\
\text { organizational culture that prizes } \\
\text { flexibility and informality and the } \\
\text { organizational needs that require } \\
\text { systems and structures to provide } \\
\text { stability and enhance learning. }\end{array}$ & $\begin{array}{l}\text { - As part a defined learning agenda, promote a culture } \\
\text { of documentation and reflection as ways to help } \\
\text { 'move' knowledge and ensure that knowledge is } \\
\text { 'institutionalized' in the organization. } \\
\text { - Provide time and space for the staff to exchange } \\
\text { knowledge, learn collectively and work in } \\
\text { cooperation. } \\
\text { - Proactively create learning opportunities for the staff. } \\
\text { Grant access to resources for skill development. } \\
\text { - Encourage an openness to change. Openly discuss } \\
\text { how to deal with growth and what steps can be taken } \\
\text { to impact on the organization's ability to continually } \\
\text { adapt and innovate. }\end{array}$ \\
\hline
\end{tabular}


Table 14: Potential Pitfalls and Potential Remedies for Enhancing Adaptive Capacity, continued

\begin{tabular}{|c|c|c|}
\hline & Potential Pitfalls & Potential Remedies \\
\hline $\begin{array}{l}\text { Systems/ } \\
\text { Evaluative } \\
\text { Thinking }\end{array}$ & $\begin{array}{l}\text { - Jumping from crisis to crisis (“fire } \\
\text { fighting") and temporarily solving } \\
\text { problems until they recur again. } \\
\text { - Encountering difficulty in defining } \\
\text { and measuring outcomes of the } \\
\text { work, especially when addressing } \\
\text { social problems where progress is } \\
\text { both hard to make and challenging } \\
\text { to measure. } \\
\text { - Evaluation is not viewed as an } \\
\text { activity that supports organizational } \\
\text { learning, but rather as something } \\
\text { that is driven by external demands } \\
\text { for accountability. Challenged to } \\
\text { implement processes and } \\
\text { standardize procedures that support } \\
\text { the use of evaluation. }\end{array}$ & $\begin{array}{l}\text { Emphasize the importance of systematically problem } \\
\text { solving and allocating time for long term planning, } \\
\text { thereby allowing pressing issues to be addressed } \\
\text { before they turn into crises. } \\
\text { - Seek out multiple points of view and feedback from } \\
\text { stakeholders. Continually incorporate outside } \\
\text { feedback through evaluation in an effort to evolve in } \\
\text { response to changing circumstances. Ensure that } \\
\text { evaluation is part of the planning and implementation } \\
\text { of organizational activities and can inform the } \\
\text { decision making process. } \\
\text { Build consensus and clearly articulate the purpose, } \\
\text { the process and the use of evaluation. Enable the } \\
\text { staff to understand how they are advancing the } \\
\text { organizational mission and achieving some type of } \\
\text { social impact. } \\
\text { Clarify staff responsibilities (including what the } \\
\text { expectations for their performance are, where they } \\
\text { should invest their time and how they can evaluate } \\
\text { their work). Make certain that there is no ambiguity } \\
\text { regarding job roles and that there is a shared } \\
\text { understanding around work procedures and } \\
\text { organizational operations. }\end{array}$ \\
\hline Social Capital & $\begin{array}{l}\text { - Tackling very challenging social } \\
\text { problems and are generally doing so } \\
\text { in pressured environments with less } \\
\text { than ideal working conditions; can } \\
\text { result in high stress or burn out. } \\
\text { - Lack of balance between allocating } \\
\text { time for the 'doing' and for } \\
\text { discussion, reflection and shared } \\
\text { learning. } \\
\text { - Logistical difficulty of coordinating } \\
\text { time together with part time staff } \\
\text { and with varying work schedules. }\end{array}$ & $\begin{array}{l}\text { - Address stresses or tensions that exist and build a } \\
\text { support system as part of the work. Nurture a sense of } \\
\text { belonging and connection among staff to the } \\
\text { organization. Create opportunities for teamwork, } \\
\text { social exchange and knowledge exchange. Support } \\
\text { both the formal and informal interaction among staff. } \\
\text { - Ensure that staff feel valued and are able to make a } \\
\text { meaningful contribution to the organization. Engage } \\
\text { staff members in the process of setting organizational } \\
\text { strategies through dialogue. Explain decisions and } \\
\text { ensure that decisions are made based on the } \\
\text { knowledge that exists in the organization. } \\
\text { - Operate with transparency: as reflected in physical } \\
\text { access (such as open office space), as well as open } \\
\text { access to information which helps to nurture a sense } \\
\text { of trust. Allow staff members to know "what's going } \\
\text { on" and to contribute to and access the knowledge } \\
\text { base of the organization. }\end{array}$ \\
\hline
\end{tabular}


Table 14: Potential Pitfalls and Potential Remedies for Enhancing Adaptive Capacity, continued

\begin{tabular}{|c|c|c|}
\hline & Potential Pitfalls & Potential Remedies \\
\hline $\begin{array}{l}\text { Social Capital, } \\
\text { cont. }\end{array}$ & & $\begin{array}{l}\text { - Schedule regular staff meetings to foster } \\
\text { collaborative work and knowledge sharing. Balancing } \\
\text { between allocating time for the 'doing' and for } \\
\text { discussion and reflection. }\end{array}$ \\
\hline $\begin{array}{l}\text { Network/ } \\
\text { External } \\
\text { Connectedness }\end{array}$ & $\begin{array}{l}\text { - Rivalry among nonprofits and } \\
\text { competition for funds and resources } \\
\text { that hamper efforts towards } \\
\text { cooperation. } \\
\text { - Lack of understanding of 'niche' } \\
\text { and how to distinguish organization } \\
\text { from other nonprofits. Becoming } \\
\text { isolated from surrounding } \\
\text { environment. } \\
\text { - Inability to overcome the lack of a } \\
\text { direct connection between the } \\
\text { 'output' of the nonprofit and the } \\
\text { resources that fund the 'output'. } \\
\text { 'Long distance' funders do not } \\
\text { necessarily have a familiarity with } \\
\text { the local landscape or an awareness } \\
\text { of the community dynamics. }\end{array}$ & $\begin{array}{l}\text { Encourage the involvement of the staff in networks } \\
\text { and cooperative activities with other nonprofits. } \\
\text { Actively support relationship building with } \\
\text { communal institutions and the participation in a } \\
\text { network of like-minded organizations. } \\
\text { - Explore opportunities for cooperation that can } \\
\text { enhance the organization's ability to achieve greater } \\
\text { impact. Consider the scope of other organizations in } \\
\text { the field when reviewing programs and policies. } \\
\text { - Investigate opportunities for local, community-based } \\
\text { fundraising. Train and involve the board in } \\
\text { fundraising and networking at the community level. } \\
\text { - Continually assess the organizational 'niche' and } \\
\text { carry out needs assessments of the community when } \\
\text { shaping goals and strategies. }\end{array}$ \\
\hline
\end{tabular}

It is possible to consider the experience of the ten selected nonprofits in the wider context of relatively new social change movement in Israel. Many of the findings from this Israeli sample of nonprofits are supported by the existing literature on nonprofits in the United States, and therefore highlight several basic elements that seem to cut across national boundaries. On the other hand, the findings also point to the particularity of the Israeli context and the fact that so many of the social change nonprofits in the country may be reaching similar stages of development. As such, there is the potential that some of the challenges faced by the nonprofits in the sample may be generalizable issues that need to be confronted by this growing sector in Israel. Certainly, the social change movement in Israel is at a critical juncture, facing a host of challenges, as well as opportunities. Without a doubt, those nonprofits with the adaptive capacity to continually evolve and sustain themselves will make an invaluable contribution to creating a civil society in Israel. 


\section{APPENDICES}

\section{APPENDIX A: ASSESSMENT QUESTIONNAIRE FOR NONPROFITS}

\section{Dear Staff Member,}

You will find enclosed a questionnaire that is part of doctoral research being carried out at University of Pittsburgh in the United States. In this questionnaire you will be asked questions related to your work environment and attitudes towards learning. It is our hope that the results of the research will be of interest to you and your organization. If you have any questions please feel free to call me at xxxxxxx.

The results of the questionnaire will be analyzed and compared across several organizations which will remain unnamed. All responses thus are confidential, both in terms of individual staff members and in terms of the organization.

Thanks you very much for your participation,

Nancy Strichman

\section{Organizational Planning}

In this section, please indicate the degree to which you agree with the following statements.

\begin{tabular}{|c|c|c|c|c|c|}
\hline $\begin{array}{l}\text { 1. We are just trying to survive day to } \\
\text { day and do not have the time to } \\
\text { consider long term plans for our } \\
\text { organization. }\end{array}$ & $\begin{array}{l}\text { Strongly } \\
\text { Disagree }\end{array}$ & Disagree & $\begin{array}{c}\text { Neither } \\
\text { Disagree nor } \\
\text { Agree }\end{array}$ & Agree & $\begin{array}{c}\text { Strongly } \\
\text { Agree }\end{array}$ \\
\hline $\begin{array}{l}\text { 2. I really feel like my work makes a } \\
\text { difference in people's lives. }\end{array}$ & $\begin{array}{l}\text { Strongly } \\
\text { Disagree }\end{array}$ & Disagree & $\begin{array}{c}\text { Neither } \\
\text { Disagree nor } \\
\text { Agree }\end{array}$ & Agree & $\begin{array}{c}\text { Strongly } \\
\text { Agree }\end{array}$ \\
\hline $\begin{array}{l}\text { 3. We are actively planning in } \\
\text { anticipation of how the external } \\
\text { environment (our client base, policy } \\
\text { changes, etc.) may change over the } \\
\text { next few years. }\end{array}$ & $\begin{array}{l}\text { Strongly } \\
\text { Disagree }\end{array}$ & Disagree & $\begin{array}{c}\text { Neither } \\
\text { Disagree nor } \\
\text { Agree }\end{array}$ & Agree & $\begin{array}{c}\text { Strongly } \\
\text { Agree }\end{array}$ \\
\hline $\begin{array}{l}\text { 4. The staff I work with have very } \\
\text { different opinions from each other } \\
\text { about where our organization needs to } \\
\text { improve. }\end{array}$ & $\begin{array}{l}\text { Strongly } \\
\text { Disagree }\end{array}$ & Disagree & $\begin{array}{c}\text { Neither } \\
\text { Disagree nor } \\
\text { Agree }\end{array}$ & Agree & $\begin{array}{c}\text { Strongly } \\
\text { Agree }\end{array}$ \\
\hline $\begin{array}{l}\text { 5. In my organization, staff understand } \\
\text { how their work relates to the goals or } \\
\text { mission of the organization. }\end{array}$ & $\begin{array}{c}\text { Strongly } \\
\text { Disagree }\end{array}$ & Disagree & $\begin{array}{c}\text { Neither } \\
\text { Disagree nor } \\
\text { Agree }\end{array}$ & Agree & $\begin{array}{c}\text { Strongly } \\
\text { Agree }\end{array}$ \\
\hline
\end{tabular}


6. In a sentence or two, please describe what you think your organization is really trying to achieve in the long term.

7. Please mark and complete one of the following sentences (that is most appropriate to your organization):

a. We would have a more cohesive organizational strategy for the future if ...

b. We have a cohesive organizational strategy because...

\section{Organizational Work Environment}

In this section, please indicate the degree to which you agree with the following statements.

\begin{tabular}{|c|c|c|c|c|c|}
\hline $\begin{array}{l}\text { 8. We have new ideas coming from all } \\
\text { levels of the organization. }\end{array}$ & $\begin{array}{l}\text { Strongly } \\
\text { Disagree }\end{array}$ & Disagree & $\begin{array}{l}\text { Neither } \\
\text { Disagree nor } \\
\quad \text { Agree }\end{array}$ & Agree & $\begin{array}{c}\text { Strongly } \\
\text { Agree }\end{array}$ \\
\hline $\begin{array}{l}\text { 9. Feedback from stakeholders in our } \\
\text { organization often lead to changes in } \\
\text { our practices. }\end{array}$ & $\begin{array}{l}\text { Strongly } \\
\text { Disagree }\end{array}$ & Disagree & $\begin{array}{l}\text { Neither } \\
\text { Disagree nor } \\
\text { Agree }\end{array}$ & Agree & $\begin{array}{c}\text { Strongly } \\
\text { Agree }\end{array}$ \\
\hline $\begin{array}{l}\text { 10. Being open to change is viewed } \\
\text { positively in this organization. }\end{array}$ & $\begin{array}{c}\text { Strongly } \\
\text { Disagree }\end{array}$ & Disagree & $\begin{array}{l}\text { Neither } \\
\text { Disagree nor } \\
\text { Agree }\end{array}$ & Agree & $\begin{array}{c}\text { Strongly } \\
\text { Agree }\end{array}$ \\
\hline $\begin{array}{l}\text { 11. In my organization, staff are } \\
\text { encouraged to offer dissenting } \\
\text { opinions and alternatives. }\end{array}$ & $\begin{array}{l}\text { Strongly } \\
\text { Disagree }\end{array}$ & Disagree & $\begin{array}{l}\text { Neither } \\
\text { Disagree nor } \\
\quad \text { Agree }\end{array}$ & Agree & $\begin{array}{c}\text { Strongly } \\
\text { Agree }\end{array}$ \\
\hline $\begin{array}{l}\text { 12. My organization provides } \\
\text { opportunities for staff to work together } \\
\text { and share knowledge. }\end{array}$ & $\begin{array}{l}\text { Strongly } \\
\text { Disagree }\end{array}$ & Disagree & $\begin{array}{l}\text { Neither } \\
\text { Disagree nor } \\
\quad \text { Agree }\end{array}$ & Agree & $\begin{array}{c}\text { Strongly } \\
\text { Agree }\end{array}$ \\
\hline $\begin{array}{l}\text { 13. We tend to work collaboratively with } \\
\text { each other. }\end{array}$ & $\begin{array}{l}\text { Strongly } \\
\text { Disagree }\end{array}$ & Disagree & $\begin{array}{l}\text { Neither } \\
\text { Disagree nor } \\
\quad \text { Agree }\end{array}$ & Agree & $\begin{array}{c}\text { Strongly } \\
\text { Agree }\end{array}$ \\
\hline $\begin{array}{l}\text { 14. We have fruitful, constructive debates } \\
\text { about new ideas. }\end{array}$ & $\begin{array}{c}\text { Strongly } \\
\text { Disagree }\end{array}$ & Disagree & $\begin{array}{l}\text { Neither } \\
\text { Disagree nor } \\
\text { Agree }\end{array}$ & Agree & $\begin{array}{c}\text { Strongly } \\
\text { Agree }\end{array}$ \\
\hline $\begin{array}{l}\text { 15. Most attempts to change important } \\
\text { things are greeted with resistance. }\end{array}$ & $\begin{array}{c}\text { Strongly } \\
\text { Disagree }\end{array}$ & Disagree & $\begin{array}{l}\text { Neither } \\
\text { Disagree nor } \\
\text { Agree }\end{array}$ & Agree & $\begin{array}{c}\text { Strongly } \\
\text { Agree }\end{array}$ \\
\hline $\begin{array}{l}\text { 16. Any practice or assumption can be } \\
\text { challenged, no matter whose it is, or } \\
\text { how long it has been in effect. }\end{array}$ & $\begin{array}{c}\text { Strongly } \\
\text { Disagree }\end{array}$ & Disagree & $\begin{array}{l}\text { Neither } \\
\text { Disagree nor } \\
\quad \text { Agree }\end{array}$ & Agree & $\begin{array}{c}\text { Strongly } \\
\text { Agree }\end{array}$ \\
\hline $\begin{array}{l}\text { 17. My organization's leadership uses } \\
\text { data/information to inform their } \\
\text { decision-making. }\end{array}$ & $\begin{array}{l}\text { Strongly } \\
\text { Disagree }\end{array}$ & Disagree & $\begin{array}{l}\text { Neither } \\
\text { Disagree nor } \\
\text { Agree }\end{array}$ & Agree & $\begin{array}{c}\text { Strongly } \\
\text { Agree }\end{array}$ \\
\hline $\begin{array}{l}\text { 18. My organizational leadership shares up } \\
\text { to date information with staff about the } \\
\text { decision making process regarding } \\
\text { organizational directions. }\end{array}$ & $\begin{array}{l}\text { Strongly } \\
\text { Disagree }\end{array}$ & Disagree & $\begin{array}{l}\text { Neither } \\
\text { Disagree nor } \\
\text { Agree }\end{array}$ & Agree & $\begin{array}{c}\text { Strongly } \\
\text { Agree }\end{array}$ \\
\hline
\end{tabular}


19. Please mark and complete one of the following sentences (that is most appropriate to your organization):

a. Staff would feel more comfortable challenging practices or offering new alternatives if...

b. Staff feels comfortable challenging practices or offering new alternatives because...

20. Please mark and complete one of the following sentences (that is most appropriate to your organization):

a. Staff would work more cooperatively together and share more knowledge if...

b. Staff works cooperatively together and shares knowledge because...

\section{Organizational Work Environment, continued}

In this section, please determine the degree to which this is something that is or is not true of your organization.

\begin{tabular}{|c|c|c|c|c|c|}
\hline $\begin{array}{l}\text { 21. In my organization, staff are } \\
\text { encouraged to ask "why" regardless } \\
\text { of rank. }\end{array}$ & $\begin{array}{l}\text { Almost } \\
\text { Never }\end{array}$ & Infrequently & Sometimes & Frequently & $\begin{array}{l}\text { Almost } \\
\text { Always }\end{array}$ \\
\hline $\begin{array}{l}\text { 22. In my organization, staff are very } \\
\text { conscious of how changes in one part } \\
\text { of the organization can affect other } \\
\text { parts. }\end{array}$ & $\begin{array}{l}\text { Almost } \\
\text { Never }\end{array}$ & Infrequently & Sometimes & Frequently & $\begin{array}{l}\text { Almost } \\
\text { Always }\end{array}$ \\
\hline $\begin{array}{l}\text { 23. In my organization, whenever staff } \\
\text { state their views, they also ask what } \\
\text { others think. }\end{array}$ & $\begin{array}{l}\text { Almost } \\
\text { Never }\end{array}$ & Infrequently & Sometimes & Frequently & $\begin{array}{l}\text { Almost } \\
\text { Always }\end{array}$ \\
\hline $\begin{array}{l}\text { 24. In my organization, staff openly } \\
\text { discuss mistakes in order to learn } \\
\text { from them. }\end{array}$ & $\begin{array}{l}\text { Almost } \\
\text { Never }\end{array}$ & Infrequently & Sometimes & Frequently & $\begin{array}{l}\text { Almost } \\
\text { Always }\end{array}$ \\
\hline $\begin{array}{l}\text { 25. When new information that would be } \\
\text { helpful to others is learned or } \\
\text { discovered, it is shared with those in } \\
\text { the organization who need it. }\end{array}$ & $\begin{array}{l}\text { Almost } \\
\text { Never }\end{array}$ & Infrequently & Sometimes & Frequently & $\begin{array}{l}\text { Almost } \\
\text { Always }\end{array}$ \\
\hline $\begin{array}{l}\text { 26. In meetings at my organization, staff } \\
\text { are encouraged to discuss the values } \\
\text { and beliefs that underlie their } \\
\text { opinions. }\end{array}$ & $\begin{array}{l}\text { Almost } \\
\text { Never }\end{array}$ & Infrequently & Sometimes & Frequently & $\begin{array}{l}\text { Almost } \\
\text { Always }\end{array}$ \\
\hline $\begin{array}{l}\text { 27. In my organization, staff are resistant } \\
\text { to having their work critiqued by } \\
\text { someone else. }\end{array}$ & $\begin{array}{l}\text { Almost } \\
\text { Never }\end{array}$ & Infrequently & Sometimes & Frequently & $\begin{array}{l}\text { Almost } \\
\text { Always }\end{array}$ \\
\hline
\end{tabular}




\begin{tabular}{|l|c|c|c|c|c|}
\hline $\begin{array}{l}\text { 28. In my organization, staff face } \\
\text { conflict over work issues in } \\
\text { productive ways. }\end{array}$ & $\begin{array}{c}\text { Almost } \\
\text { Never }\end{array}$ & Infrequently & Sometimes & Frequently & $\begin{array}{c}\text { Almost } \\
\text { Always }\end{array}$ \\
\hline $\begin{array}{l}\text { 29. My organization's leadership is open } \\
\text { to constructive, critical feedback } \\
\text { from the staff. }\end{array}$ & $\begin{array}{c}\text { Almost } \\
\text { Never }\end{array}$ & Infrequently & Sometimes & Frequently & $\begin{array}{c}\text { Almost } \\
\text { Always }\end{array}$ \\
\hline
\end{tabular}

30. Please mark and complete one of the following sentences (that is most appropriate to your organization):

a. Staff would feel more comfortable discussing mistakes, receiving and/ or giving open and honest feedback to each other if ...

b. Staff feels comfortable discussing mistakes, receiving and/ or giving open and honest feedback to each other because...

31. Please mark and complete one of the following sentences (that is most appropriate to your organization):

a. Staff would face conflict over work issues in productive ways if ...

b. Staff faces conflict over work issues in productive ways because...

\section{Organizational Assessment of Progress}

In this section, please indicate the degree to which you agree with the following statements.

\begin{tabular}{|l|c|c|c|c|c|}
\hline $\begin{array}{l}\text { 32. Information is gathered from } \\
\text { stakeholders to gauge how well we're } \\
\text { doing. }\end{array}$ & $\begin{array}{c}\text { Strongly } \\
\text { Disagree }\end{array}$ & Disagree & $\begin{array}{c}\text { Neither } \\
\text { Disagree nor } \\
\text { Agree }\end{array}$ & Agree & $\begin{array}{c}\text { Strongly } \\
\text { Agree }\end{array}$ \\
\hline $\begin{array}{l}\text { 33. Currently available information tells us } \\
\text { that we need to know about the } \\
\text { effectiveness of our programs. }\end{array}$ & $\begin{array}{c}\text { Strongly } \\
\text { Disagree }\end{array}$ & Disagree & $\begin{array}{c}\text { Neither } \\
\text { Disagree nor } \\
\text { Agree }\end{array}$ & Agree & $\begin{array}{c}\text { Strongly } \\
\text { Agree }\end{array}$ \\
\hline $\begin{array}{l}\text { 34. In my organization, staff continually } \\
\text { ask themselves how they're doing, } \\
\text { what they can do better, and what is } \\
\text { working. }\end{array}$ & $\begin{array}{c}\text { Strongly } \\
\text { Disagree }\end{array}$ & Disagree & $\begin{array}{c}\text { Nisagree nor } \\
\text { Agree }\end{array}$ & Agree & $\begin{array}{c}\text { Strongly } \\
\text { Agree }\end{array}$ \\
\hline $\begin{array}{l}\text { 35. Evaluative activity is seen as } \\
\text { threatening the status quo. }\end{array}$ & $\begin{array}{l}\text { Strongly } \\
\text { Disagree }\end{array}$ & Disagree & $\begin{array}{c}\text { Aisagree nor } \\
\text { Agree }\end{array}$ & Agree & $\begin{array}{c}\text { Strongly } \\
\text { Agree }\end{array}$ \\
\end{tabular}


36. Please mark and complete one of the following sentences (that is most appropriate to your organization):

a. We would carry out more evaluation if...

b. We carry out evaluation because...

\section{Relationship with Other Organizations}

In this section, please indicate the degree to which you agree with the following statements.

\begin{tabular}{|l|c|c|c|c|c|}
\hline $\begin{array}{l}\text { 37. It is worthwhile to work with other } \\
\text { organizations because it allows us to } \\
\text { achieve more that we could } \\
\text { individually as an organization. }\end{array}$ & $\begin{array}{c}\text { Strongly } \\
\text { Disagree }\end{array}$ & Disagree & $\begin{array}{c}\text { Neither } \\
\text { Disagree } \\
\text { Agree }\end{array}$ & Agree & $\begin{array}{c}\text { Strongly } \\
\text { Agree }\end{array}$ \\
\hline $\begin{array}{l}\text { 38. When we review our programs and } \\
\text { policies, we routinely consider what } \\
\text { other organizations in our field are } \\
\text { doing. }\end{array}$ & $\begin{array}{c}\text { Strongly } \\
\text { Disagree }\end{array}$ & Disagree & $\begin{array}{c}\text { Disagree nor } \\
\text { Agree }\end{array}$ & Agree & $\begin{array}{c}\text { Strongly } \\
\text { Agree }\end{array}$ \\
\hline $\begin{array}{l}\text { 39. We actively participate in a network of } \\
\text { like-minded organizations in order to } \\
\text { exchange knowledge. }\end{array}$ & $\begin{array}{c}\text { Strongly } \\
\text { Disagree }\end{array}$ & Disagree & $\begin{array}{c}\text { Disagree nor } \\
\text { Agree }\end{array}$ & Agree & $\begin{array}{c}\text { Strongly } \\
\text { Agree }\end{array}$ \\
\hline
\end{tabular}

40. Please mark and complete one of the following sentences (that is most appropriate to your organization):

a. We would proactively cooperate with other like-minded nonprofits organizations more frequently if...

b. We proactively cooperate with other like-minded nonprofits organizations because...

\section{Additional Comments:}




\section{APPENDIX B: DEVELOPMENT OF ASSESSMENT QUESTIONNAIRE}

\section{Drawn from the following assessment surveys:}

- ROLE - The Readiness for Organizational Learning and Evaluation Instrument (Preskill \& Torres, 2000b)

- ALCS - Assessing Learning Culture Scale Items (Botcheva, White \& Huffman, 2002)

- DLOQ - Dimensions of the Learning Organization Questionnaire (DLOQ), (Watkins \& Marsick, 1999)

- ELOC - Evaluation of Learning Capacity (Davidson, 2001)

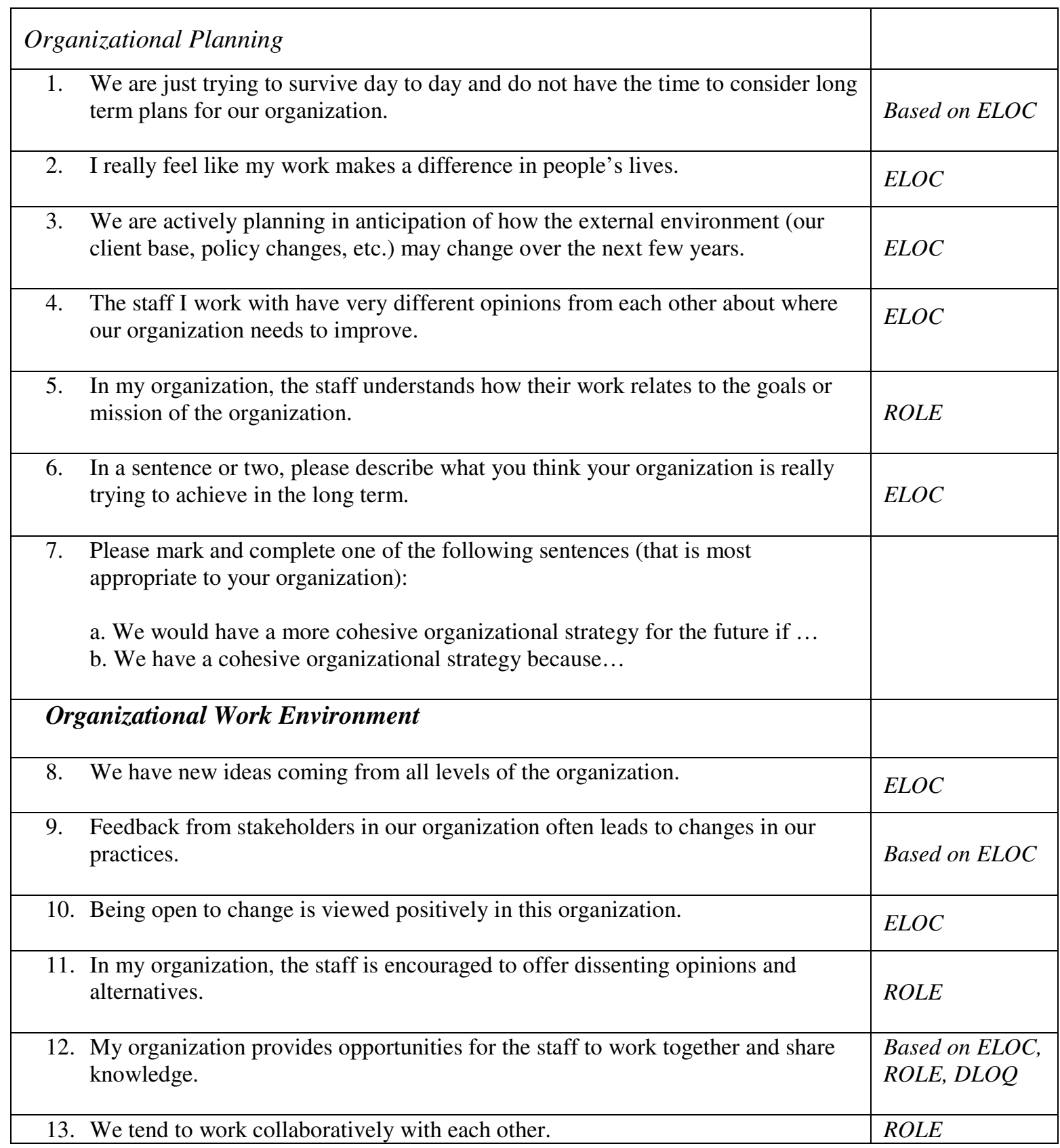




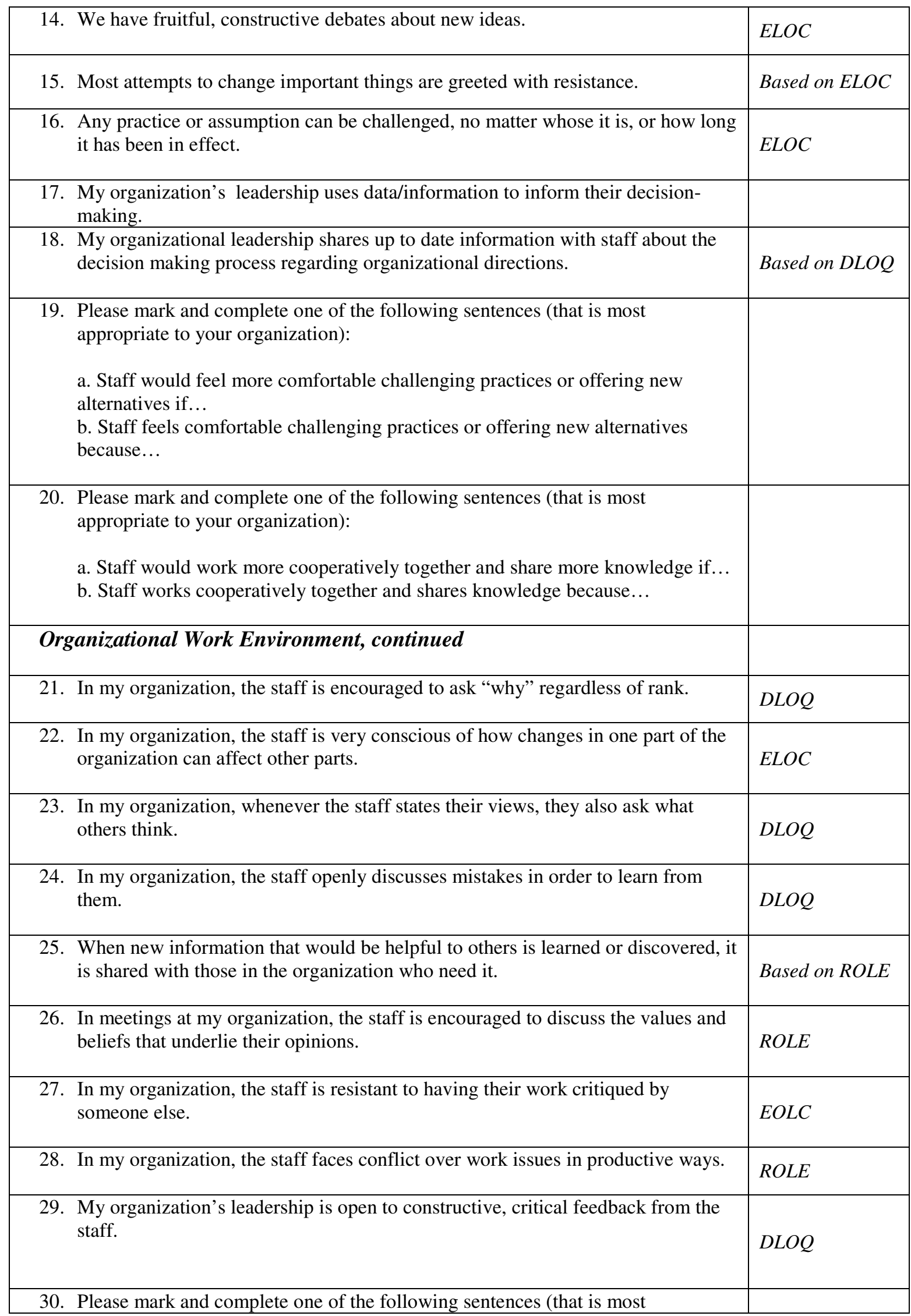




\begin{tabular}{|c|c|}
\hline $\begin{array}{l}\text { appropriate to your organization): } \\
\text { a. Staff would feel more comfortable discussing mistakes, receiving and/ or } \\
\text { giving open and honest feedback to each other if ... } \\
\text { b. Staff feels comfortable discussing mistakes, receiving and/ or giving open and } \\
\text { honest feedback to each other because... }\end{array}$ & \\
\hline $\begin{array}{l}\text { 31. Please mark and complete one of the following sentences (that is most } \\
\text { appropriate to your organization): } \\
\text { a. Staff would face conflict over work issues in productive ways if ... } \\
\text { b. Staff faces conflict over work issues in productive ways because... }\end{array}$ & \\
\hline Organizational Assessment of Progress & \\
\hline 32. Information is gathered from stakeholders to gauge how well we're doing. & Based on ROLE \\
\hline $\begin{array}{l}\text { 33. Currently available information tells us that we need to know about the } \\
\text { effectiveness of our programs. }\end{array}$ & Based on ROLE \\
\hline $\begin{array}{l}\text { 34. In my organization, the staff continually asks themselves how they're doing, } \\
\text { what they can do better, and what is working. }\end{array}$ & $R O L E$ \\
\hline 35. Evaluative activity is seen as threatening the status quo. & $A L C S$ \\
\hline $\begin{array}{l}\text { 36. Please mark and complete one of the following sentences (that is most } \\
\text { appropriate to your organization): } \\
\text { a. We would carry out more evaluation if... } \\
\text { b. We carry out evaluation because... }\end{array}$ & $A L C S$ \\
\hline Relationship with Other Organizations & \\
\hline $\begin{array}{l}\text { 37. It is worthwhile to work with other organizations because it allows us to achieve } \\
\text { more that we could individually as an organization. }\end{array}$ & \\
\hline $\begin{array}{l}\text { 38. When we review our programs and policies, we routinely consider what other } \\
\text { organizations in our field are doing. }\end{array}$ & Based on ELOC \\
\hline $\begin{array}{l}\text { 39. We actively participate in a network of like-minded organizations in order to } \\
\text { exchange knowledge. }\end{array}$ & \\
\hline $\begin{array}{l}\text { 40. Please mark and complete one of the following sentences (that is most } \\
\text { appropriate to your organization): } \\
\text { a. We would proactively cooperate with other like-minded nonprofits } \\
\text { organizations more frequently if... } \\
\text { b. We proactively cooperate with other like-minded nonprofits organizations } \\
\text { because... }\end{array}$ & \\
\hline
\end{tabular}




\section{APPENDIX C: INTERVIEW GUIDE FOR STAFF AND BOARD}

Thank you for agreeing to talk with me about your work environment at your organization. We appreciate the time you are contributing to our research study. Your responses to the questions are confidential. We will not report findings in any way that can identify you. Please feel free to be candid in your responses.

1. Please tell us about your organizations and its goals. What are you trying to achieve?

2. What brought you to this particular organization?

3. Do you feel a strong connection to the vision of the nonprofit? Do you feel that your work makes a difference in people's lives? If so, why, if not, why not?

4. Do you feel that the organization plans for the future? Does your organization have a cohesive organizational strategy? If so, why, if not, why not?

5. Have you set specific targets for these goals of the organization for the coming 1 or 2 years?

6. How would you define success for your organization in relation to each target outcome?

7. How do you check if you are achieving these targets?

8. What are the biggest challenges to achieving the long term goals of the organization?

9. What are major changes that have occurred in this organization in the last five years? How do you think the organization plans for and responds to change?

10. What changes do you see coming in the next few years and how do you know they are coming?

11. How do you ensure that you are meeting the needs of your community base?

12. How would you describe your organization's 'character'? How would you distinguish it from other organizations? What makes your organization "special"?

13. Do you feel encouraged to take initiatives? Do you feel comfortable challenging practices or offering new alternatives? If so, why. If not, why not?

14. Does the staff work cooperatively with each other and share knowledge? If so, why. If not, why not?

15. Do you feel comfortable discussing mistakes? Do you feel comfortable receiving/giving feedback to each other? If so why? If not, why not?

16. Does the staff face conflict over work issues in productive ways? If so, why. If not, why not?

17. Do you collaborate with other organizations? If so why? If not, why not?

18. What do you need to build the capacity of your organization? Are there specific things that could be done internally to strengthen your organization's capacity to fulfill its mission? 


\section{APPENDIX D: FRAMEWORK FOR ASSESSMENT OF ADAPTIVE CAPACITY}

\begin{tabular}{|c|c|}
\hline $\begin{array}{l}\text { Shared Vision/ } \\
\text { Shared } \\
\text { Meaning }\end{array}$ & $\begin{array}{l}\text { I really feel like my work makes a difference in people's lives. (\#2) } \\
\text { The staff I work with have very different opinions from each other about where our organization needs } \\
\text { to improve. (\# 4- reverse coded) } \\
\text { In my organization, staff understand how their work relates to the goals or mission of the organization. } \\
\text { (\# 5) } \\
\text { Our goal as an organization is } \\
\text { My organizational leadership shares up to date information with staff about the decision making } \\
\text { process regarding organizational directions (\#18). } \\
\text { Future Scanning } \\
\text { We are just trying to survive day to day and do not have the time to consider long term plans for our } \\
\text { organization. (\#1 - reverse coded) } \\
\text { We are actively planning in anticipation of how the external environment (our client base, policy } \\
\text { changes, etc.) may change over the next few years. (\#3) }\end{array}$ \\
\hline $\begin{array}{l}\text { Inquisitiveness/ } \\
\text { Openness }\end{array}$ & $\begin{array}{l}\text { Open to Change } \\
\text { Being open to change is viewed positively in this organization. (\#10) } \\
\text { Most attempts to change important things are greeted with resistance. (\#15- reverse coded) } \\
\text { Challenge assumptions } \\
\text { Any practice or assumption can be challenged, no matter whose it is, or how long it has been in effect. } \\
\text { (\#16) } \\
\text { In my organization, staff are encouraged to ask "why" regardless of rank. (\#21) } \\
\text { Take risks, discuss mistakes } \\
\text { In my organization, staff openly discuss mistakes in order to learn from them. (\#24) }\end{array}$ \\
\hline $\begin{array}{l}\text { Systems } \\
\text { thinking } \\
\text { Evaluative } \\
\text { Thinking }\end{array}$ & $\begin{array}{l}\text { Systems and encouragement for sharing knowledge } \\
\text { My organization provides opportunities for staff to work together and share knowledge. (\#12) } \\
\text { When new information that would be helpful to others is learned or discovered, it is shared with those } \\
\text { in the organization who need it. (\#25) } \\
\text { Interrelatedness }\end{array}$ \\
\hline
\end{tabular}




\begin{tabular}{|c|c|}
\hline & $\begin{array}{l}\text { In my organization, staff are very conscious of how changes in one part of the organization can affect } \\
\text { other parts. (\#22) } \\
\text { Evaluative Attitude } \\
\text { Information is gathered from stakeholders to gauge how well we're doing. (\#32) } \\
\text { Currently available information tells us that we need to know about the effectiveness of our programs. } \\
\text { (\#33) } \\
\text { In my organization, staff continually ask themselves how they're doing, what they can do better, and } \\
\text { what is working. (\#34) } \\
\text { Evaluative activity is seen as threatening the status quo. (\#35)- reverse coded } \\
\text { My organization's leadership uses data/information to inform their decision-making. (\#17) }\end{array}$ \\
\hline $\begin{array}{l}\text { A Community } \\
\text { of Learners : } \\
\text { Informal } \\
\text { Learning/ } \\
\text { Social Capital }\end{array}$ & $\begin{array}{l}\text { Dialogue } \\
\text { We have fruitful, constructive debates about new ideas. (\#14) } \\
\text { In my organization, whenever staff state their views, they also ask what others think.(\#23) } \\
\text { In meetings at my organization, staff are encouraged to discuss the values and beliefs that underlie } \\
\text { their opinions. (\#26) } \\
\frac{\text { Critical feedback }}{\text { In my organization, staff are resistant to having their work critiqued by someone else. (\#27)- reverse }} \\
\text { coded } \\
\text { My organization's leadership is open to constructive, critical feedback from the staff. (\#29) } \\
\text { Enhancing Cooperation } \\
\text { In my organization, staff face conflict over work issues in productive ways (\#28). } \\
\text { We tend to work collaboratively with each other. (\#13) }\end{array}$ \\
\hline $\begin{array}{l}\text { External } \\
\text { Focus/ } \\
\text { Network } \\
\text { Connectedness }\end{array}$ & $\begin{array}{l}\text { We are actively planning in anticipation of how the external environment (our client base, policy } \\
\text { changes, etc.) may change over the next few years. (\#3 ) (counted twice) } \\
\text { Feedback from stakeholders in our organization often lead to changes in our practices. (\#9) (counted } \\
\text { twice) } \\
\text { Information is gathered from stakeholders to gauge how well we're doing. (\#32) (counted twice) } \\
\text { It is worthwhile to work with other organizations because it allows us to achieve more that we could } \\
\text { individually as an organization. (\#37) } \\
\text { When we review our programs and policies, we routinely consider what other organizations in our } \\
\text { field are doing. (\#38) } \\
\text { We actively participate in a network of like-minded organizations in order to exchange knowledge. } \\
\text { (\#39) }\end{array}$ \\
\hline
\end{tabular}




\section{APPENDIX E: CODING CHART OF QUALITATIVE DATA AND SAMPLE INTERVEWS}

\begin{tabular}{|c|c|}
\hline Adaptive Capacity & Description of Concepts \\
\hline 1. Shared Vision & $\begin{array}{l}\text { a. Creating shared understanding, collectively building a shared purpose. } \\
\text { Staff involved in setting, owning and implementing a joint vision. } \\
\text { b. Commitment - connect to personal vision and feeling of genuine } \\
\text { excitement or inspired by work } \\
\text { c. Encouraging of group dialogue, communication and collective reflection } \\
\text { d. Question of shared understanding about direction of organization or gap } \\
\text { between current and desired organization performance } \\
\text { e. Understand relationship of work to organizational goals }\end{array}$ \\
\hline $\begin{array}{l}\text { 2. Inquisitiveness/ } \\
\text { Openness }\end{array}$ & $\begin{array}{l}\text { a. Willingness to question underlying assumptions and accepted wisdom. } \\
\text { No 'sacred cows' or undiscussables. (overlap to collective learning) } \\
\text { b. Rewarding curiosity, risk taking and experimentation } \\
\text { c. Nurturing a safe environment for 'failure'. Learning collectively from } \\
\text { past mistakes. Discussions focus not only on success or noncritical } \\
\text { problems. Mistakes not lead to blame. } \\
\text { d. Creating and implementing new ideas, a 'marketplace' for ideas, } \\
\text { e. Embracing 'dissension' and diversity of thought } \\
\text { f. 'Probing' the future, awareness of possible changes in the future } \\
\text { g. Change viewed as positive and natural, scanning, openness } \\
\text { h. Diversity of thought valued, participatory decision making }\end{array}$ \\
\hline $\begin{array}{c}\text { 3. Systems / } \\
\text { Evaluative } \\
\text { Thinking }\end{array}$ & $\begin{array}{l}\text { a. An "appetite for inquiry": seek out data and information in order to learn, } \\
\text { and then apply and share the knowledge. } \\
\text { b. Data collection, learning and knowledge development are an essential, } \\
\text { organization -wide effort. } \\
\text { c. Evaluative activities are considered as a tool for learning and improving } \\
\text { performance. } \\
\text { d. Systems exist to capture and share learning- open access to information. } \\
\text { emphasis on communication and dissemination of information. } \\
\text { e. Understanding interdependence of different parts of organization, } \\
\text { f. Adopting long term approach to solving problems. Recognizing patterns } \\
\text { of change/ Addressing underlying causes of events/ Acknowledging the } \\
\text { nature of unpredictability }\end{array}$ \\
\hline 4. Social Capital & $\begin{array}{l}\text { a. Promotion of inquiry and dialogue. Value of communication and quality } \\
\text { of ideas over hierarchy and status, inclusive decision-making processes. } \\
\text { Encourages the open exchange of ideas and discusses norms and } \\
\text { expectations. } \\
\text { b. Conversations invite challenge or inquiry for understanding, dialogue, } \\
\text { and active listening of views of others. } \\
\text { c. Valuing reflection as well as action. Has time for reflection and feedback } \\
\text { built in to planning. Redefining the definition of "productive" work } \\
\text { d. Continuous opportunities for learning -learning designed into the work } \\
\text { e. Cultivating a collaborative work environment and problem solving that is } \\
\text { valued and rewarded. Signaling the importance of knowledge sharing and }\end{array}$ \\
\hline
\end{tabular}




\begin{tabular}{|c|c|}
\hline & $\begin{array}{l}\text { importance of reciprocity. Rewarding group success, not just individual. } \\
\text { Expectation of staff to work together. } \\
\text { freating opportunities for interaction (providing both time and space). } \\
\text { Supporting the creation of social networks. Investing in personal and } \\
\text { professional development. } \\
\text { g. Creating an environment of trust among staff. Environment to encourage } \\
\text { critical feedback and seek truth about own performance Ensuring that } \\
\text { organizational policies nurture trust }\end{array}$ \\
\hline $\begin{array}{l}\text { 5. External Focus/ } \\
\text { Network } \\
\text { Connectedness }\end{array}$ & $\begin{array}{l}\text { a. Connected to external environment. Awareness of interdependence with } \\
\text { surrounding environment. "Sufficiently porous" to information and ideas } \\
\text { from outside the organization. } \\
\text { b. Locates resources and capacities from outside of organization. } \\
\text { c. Recognition of importance of network and community/ Construction of } \\
\text { strategic partnerships or affiliations with other organizations and } \\
\text { colleagues/ Development of networks to achieve mission } \\
\text { d. Understanding needs of clients or other organizational stakeholders, } \\
\text { Board brings in ideas } \\
\text { e. Understanding of potential to create systematic change through strategic } \\
\text { alliances and joint efforts with other organizations. }\end{array}$ \\
\hline
\end{tabular}

\section{Interview with Director}

Informal atmosphere, not hierarchical, not a big organization but a small team. 4e

The staff believe in our goals, with a connection to the place. Not a classic view of management that workers give reports, but make room for all of the team to be a part and to make sure that the majority builds consensus. There are defined jobs but lots of room for imagination and initiative.

\section{$\mathbf{1 a}, 2 \mathbf{2 h}, \mathbf{4 e}$}

Shared vision, and shared values but there are nuances and differences - all agree to improve the situation, all agree on priorities, lots of discussion on how to achieve this. 1a, 1d

Not easy always to implement changes, receive ideas and suggestions on how to improve and important for the staff to get feedback from those activist in the field but not always able to do what is suggested, the field staff always report back on suggestions. - for example, too focused on Haifa so now opening a branch in the center. $\mathbf{2 d}, \mathbf{2 f}$

Another example - feedback from a foundation to go back to former participants and to check the long term impact of the work. (not just to check the impact at the end of the project but follow up a few years later). Process of dialogue with foundations is good- it does not work when it is an ultimatum from the foundation. $2 \mathrm{~d}, 3 \mathbf{3}, \mathbf{5 a}$

Always working to improve communication but a challenges when the field coordinators is not working here - staff and then the field people are dispersed geographically and there is not enough money to have meetings more frequently then once a month ( and pay for transportation), so by email. Geographical issues is a challenge - there is natural communication between the 3 out of 4 that are here all the time. Always try to have staff meetings once a week. Also language challenge - hold meetings in English - but not all express themselves as well in English. 1c,1d,3e 
Hope as much as possible to cooperate with other organizations, it is good for our strategy and also it makes sense practically because we cannot fund all of our activities. And we only own this one building, so work with existing organizations, community centers, schools, etc. and invite other organizations to take part in our activities, part of our goal for building youth leadership. Not always smooth but it usually works. 5a,5b,5c,5e

\section{Board Member}

Organization changes all the time. All range of political spectrum on board. Started as modest organizations with one room, one computer. $2 \mathrm{~g}$

Sought to be an example of volunteering for our community. 1a

Volunteered as a member and then asked on the on board. - still naïve in a non-naïve world, ideology keeps us together. 1a

A retreat in 2002, given a model of nonprofits - division of authority, roles, of board, staff, structure of organization, what do we want, what are the goals, what are our tools, where see selves in 10 years, became clear to everyone thru this discussion. Retreat - involved, felt connected to the vision, ready to take more responsibility. Why so together? - take things simply, look at whole picture, stay focused on the purpose and goals, great desire to succeed, belief in the goals and being responsible and willing to give in $\mathbf{1 a}, \mathbf{1 c}, 1 \mathrm{1d}, \mathbf{2 a}, \mathbf{4 f}$

Board involved in who leads trips abroad, interview who is hired, supervisor of leadership program for students and secretary. Very involved but not in the everyday work of the staff, think of vision and initiating new ideas. Saw in the results that the retreat helped, continued to grow, become more well known, etc. $\mathbf{1 a}, \mathbf{1 c}, \mathbf{1 d}, \mathbf{4 f}+\mathbf{3 b}$

In 2005, had another retreat, initiated by board. Decided should focus also on vision, and examine existing staff structure. , how to be more efficient, make it better. 1a,1c,1d,4f

Determined that the board needs to initiate more, be more personally involved in activities, asked leadership coordinator to give more reports, monthly newsletter- determined that the editor decides within boundaries, what goes in - discussion that his decision, not the board's, $\mathbf{3 b}, \mathbf{3 d}$

The strength of the organization is its connection to the goals vision, and prose, fear that as continues to grow -will lose this naivety and no longer be the case, people come with a true ideology and commitment. Challenges of where to open a new branch, whom to trust politically complicated to open in certain places. In the past made mistakes trusting people - talk about it - the board hears about things because part of larger network. 1e,5c,2f

$3^{\text {rd }}$ year of project, didn't have a measurement - so is developing a tool to measure impact over time and see feedback of participants and if succeeded - came out of retreat

Board is young and also in spirit - can talk to youth and understand them - see how can change ourselves to help change our communities and families, $\mathbf{3 a}, \mathbf{3 b}, \mathbf{3 c}, \mathbf{3 d}$

\section{$\underline{\text { Staff Member }}$}

"Small organization doing big things". Can lose important things when trying to be big. 
Feedback is welcomed, and constructive criticism of others work. Also a spirit, youth a feeling of looseness and freedom. Close relation with target population. Don't feel like a office - mix social and work.

Not feel being watched or observed or a limit of what can and can't do. 1c,4a,4e,4f,4g,5a

Feel trusted - so makes you want to work more because feel faith in you and a belonging to the organization. There is no gossip - all concerned about the project or work. Work with other orgs, but not feel competition, keeps a positive connection with other NGOs. Has reputation of good, peaceful organization, proud to be a part of it. 1a,1e,4g,5a, 5c, $2 \mathrm{c}$

Share ideas about own work and the whole organization. The open spirit enables it. What is say is important and whether agree or disagree is a different issue but no secrets, feel free to share. 1a,1c,1e,4a

Gives new ideas, feels it is appreciated and not just dialogue but also see impact of ideas, can bring about change. Positive flexibility about meetings $\mathbf{1 a}, \mathbf{4 d}, \mathbf{4 e}, \mathbf{4 f}$

Always wanting to developing the organization, not a final space - ' not good enough'; we want to improve all the time. $2 \mathrm{f}, \mathbf{2 g}, \mathbf{3 f}$

All kinds of feedback - through monthly meetings with the group leaders and coordinators. Know each other - discuss, and at end of years leaders are asked to use evaluation tool with their group and must document it and write evaluation. $\mathbf{3 b}, \mathbf{3 c}, \mathbf{3 d}$

Previous problems of staff instability - coordinator left after 2 months, then 3 months - hurt the project now must write a paper of responsibility, job description, and also for volunteers. A group decision because found difficult to know range of limits of the job

\section{$1 a, 2 c, 2 g, 3 f, 4 a, 4 e$}

Strategic planning - looking as long term, not just per project, according to funding but at ultimate long terms goal. What do we want for ourselves in 10 years - the meetings was very powerful - the retreat. Staff, facilitators, board and community leaders were involved in the planning. And since have had 4 meetings on our strategy. $\mathbf{2 f}, \mathbf{3 f}, \mathbf{4 a}, \mathbf{4 c}, \mathbf{4 f}, \mathbf{5 b}, \mathbf{5 c}, \mathbf{5 d}$

We have suggestion to cooperate in projects - good for networking but not always worth it for us. Responsibility falls on staff. Festival etc. but began to ask how it improves our work, if suggested to us ? emerged from strategic planning, became more focused, now set limits - developing selves. Now at a different phase where these projects can not only distract us but harm us because staff is taken away from doing the work. 1e,2f,2g

We do not feel threatened by other orgs or overprotective of our work. . work is for the community. 1e,5a,5e

Want to keep $15 \%$ of us always stay un-established. We are $85 \%$ established. 1a,2g

Biggest difficulty is funding that restricts our work. Small organization doing major things, need more staff. Lack administrative assistant. , help with auditing. Did not want the face of a secretary because of organization not perceived as an office, but decided that is now necessary. $\mathbf{1 d , 2 c , 2 f}$

Came to projects - saw unprofessional things - information about leaders not provided on time - challenge for next year is to work with existing resources. 1e,2a,2c,2f,3f 


\title{
APPENDIX F: PILOT QUESTIONNAIRE
}

\author{
Dear Staff Member,
}

You will find enclosed a questionnaire that is part of doctoral research being carried out at University of Pittsburgh in the United States. The anonymous survey, which will be asking questions related to your work environment and attitudes towards learning, is being circulated among several social change nonprofits in Haifa. It is our hope that the results of the research will be of interest to you and your organization. If you have any questions please feel free to call me at xxxxxxx. Please notice that at the end of the survey there is extra space for additional comments. The results of the questionnaire will be analyzed and compared across several organizations that will remain unnamed. All responses thus are confidential, both in terms of individual staff members and in terms of the organization. At the same time, each organization will receive its own organizational profile and summary of the results.

Thanks you very much for your participation,

Nancy Strichman

\begin{tabular}{|c|c|c|c|c|c|}
\hline $\begin{array}{l}\text { Organizational Planning } \\
\text { In this section, please indicate the degree to wh }\end{array}$ & you agr & the fo & ing staten & & \\
\hline $\begin{array}{l}\text { 41. We are just trying to survive day to day } \\
\text { and do not have the time to consider long } \\
\text { term plans for our organization. }\end{array}$ & $\begin{array}{c}\text { Strongly } \\
\text { Disagree }\end{array}$ & Disagree & $\begin{array}{l}\text { Neither } \\
\text { Disagree nor } \\
\text { Agree }\end{array}$ & Agree & $\begin{array}{l}\text { Strongly } \\
\text { Agree }\end{array}$ \\
\hline $\begin{array}{l}\text { 42. I really feel like my work makes a } \\
\text { difference in people's lives. }\end{array}$ & $\begin{array}{l}\text { Strongly } \\
\text { Disagree }\end{array}$ & Disagree & $\begin{array}{l}\text { Neither } \\
\text { Disagree nor } \\
\quad \text { Agree }\end{array}$ & Agree & $\begin{array}{c}\text { Strongly } \\
\text { Agree }\end{array}$ \\
\hline $\begin{array}{l}\text { 43. We are actively planning in anticipation of } \\
\text { how the external environment (our client } \\
\text { base, policy changes, etc.) may change } \\
\text { over the next few years. }\end{array}$ & $\begin{array}{c}\text { Strongly } \\
\text { Disagree }\end{array}$ & Disagree & $\begin{array}{l}\text { Neither } \\
\text { Disagree nor } \\
\quad \text { Agree }\end{array}$ & Agree & $\begin{array}{c}\text { Strongly } \\
\text { Agree }\end{array}$ \\
\hline $\begin{array}{l}\text { 44. The staff I work with have very different } \\
\text { opinions from each other about where our } \\
\text { organization needs to improve. }\end{array}$ & $\begin{array}{c}\text { Strongly } \\
\text { Disagree }\end{array}$ & Disagree & $\begin{array}{l}\text { Neither } \\
\text { Disagree nor } \\
\quad \text { Agree }\end{array}$ & Agree & $\begin{array}{c}\text { Strongly } \\
\text { Agree }\end{array}$ \\
\hline $\begin{array}{l}\text { 45. In my organization, staff understand how } \\
\text { their work relates to the goals or mission of } \\
\text { the organization. }\end{array}$ & $\begin{array}{l}\text { Strongly } \\
\text { Disagree }\end{array}$ & Disagree & $\begin{array}{l}\text { Neither } \\
\text { Disagree nor } \\
\quad \text { Agree }\end{array}$ & Agree & $\begin{array}{c}\text { Strongly } \\
\text { Agree }\end{array}$ \\
\hline $\begin{array}{l}\text { 46. In a sentence or two, please describe what } \\
\text { you think your organization is really trying } \\
\text { to achieve in the long term. }\end{array}$ & & & & & \\
\hline $\begin{array}{l}\text { Organizational Work Environment } \\
\text { In this section, please indicate the degree to whi }\end{array}$ & . & we $J$ & ng stalen & & \\
\hline $\begin{array}{l}\text { 47. We have new ideas coming from all levels } \\
\text { of the organization. }\end{array}$ & $\begin{array}{l}\text { Strongly } \\
\text { Disagree }\end{array}$ & Disagree & $\begin{array}{l}\text { Neither } \\
\text { Disagree nor } \\
\quad \text { Agree }\end{array}$ & Agree & $\begin{array}{c}\text { Strongly } \\
\text { Agree }\end{array}$ \\
\hline $\begin{array}{l}\text { 48. Feedback from stakeholders in our } \\
\text { organization often lead to changes in our } \\
\text { practices. }\end{array}$ & $\begin{array}{c}\text { Strongly } \\
\text { Disagree }\end{array}$ & Disagree & $\begin{array}{l}\text { Neither } \\
\text { Disagree nor } \\
\text { Agree }\end{array}$ & Agree & $\begin{array}{c}\text { Strongly } \\
\text { Agree }\end{array}$ \\
\hline
\end{tabular}




\begin{tabular}{|c|c|c|c|c|c|}
\hline $\begin{array}{l}\text { 49. Being open to change is viewed positively } \\
\text { in this organization. }\end{array}$ & $\begin{array}{l}\text { Strongly } \\
\text { Disagree }\end{array}$ & Disagree & $\begin{array}{l}\text { Neither } \\
\text { Disagree nor } \\
\text { Agree }\end{array}$ & Agree & $\begin{array}{c}\text { Strongly } \\
\text { Agree }\end{array}$ \\
\hline $\begin{array}{l}\text { 50. In my organization, staff are encouraged to } \\
\text { offer dissenting opinions and alternatives. }\end{array}$ & $\begin{array}{l}\text { Strongly } \\
\text { Disagree }\end{array}$ & Disagree & $\begin{array}{l}\text { Neither } \\
\text { Disagree nor } \\
\text { Agree }\end{array}$ & Agree & $\begin{array}{c}\text { Strongly } \\
\text { Agree }\end{array}$ \\
\hline $\begin{array}{l}\text { 51. My organization provides opportunities for } \\
\text { staff to work together and share } \\
\text { knowledge. }\end{array}$ & $\begin{array}{l}\text { Strongly } \\
\text { Disagree }\end{array}$ & Disagree & $\begin{array}{l}\text { Neither } \\
\text { Disagree nor } \\
\text { Agree }\end{array}$ & Agree & $\begin{array}{c}\text { Strongly } \\
\text { Agree }\end{array}$ \\
\hline $\begin{array}{l}\text { 52. We tend to work collaboratively with each } \\
\text { other. }\end{array}$ & $\begin{array}{l}\text { Strongly } \\
\text { Disagree }\end{array}$ & Disagree & $\begin{array}{l}\text { Neither } \\
\text { Disagree nor } \\
\text { Agree }\end{array}$ & Agree & $\begin{array}{c}\text { Strongly } \\
\text { Agree }\end{array}$ \\
\hline $\begin{array}{l}\text { 53. We have fruitful, constructive debates } \\
\text { about new ideas. }\end{array}$ & $\begin{array}{l}\text { Strongly } \\
\text { Disagree }\end{array}$ & Disagree & $\begin{array}{l}\text { Neither } \\
\text { Disagree nor } \\
\text { Agree }\end{array}$ & Agree & $\begin{array}{c}\text { Strongly } \\
\text { Agree }\end{array}$ \\
\hline $\begin{array}{l}\text { 54. Most attempts to change important things } \\
\text { are greeted with resistance. }\end{array}$ & $\begin{array}{l}\text { Strongly } \\
\text { Disagree }\end{array}$ & Disagree & $\begin{array}{l}\text { Neither } \\
\text { Disagree nor } \\
\text { Agree }\end{array}$ & Agree & $\begin{array}{c}\text { Strongly } \\
\text { Agree }\end{array}$ \\
\hline $\begin{array}{l}\text { 55. Any practice or assumption can be } \\
\text { challenged, no matter whose it is, or how } \\
\text { long it has been in effect. }\end{array}$ & $\begin{array}{l}\text { Strongly } \\
\text { Disagree }\end{array}$ & Disagree & $\begin{array}{l}\text { Neither } \\
\text { Disagree nor } \\
\text { Agree }\end{array}$ & Agree & $\begin{array}{c}\text { Strongly } \\
\text { Agree }\end{array}$ \\
\hline \multicolumn{6}{|c|}{$\begin{array}{l}\text { Organizational Work Environment, continued } \\
\text { In this section, please determine the degree to which this is something that is or is not true of your organization. }\end{array}$} \\
\hline & $\begin{array}{l}\text { Almost } \\
\text { Never }\end{array}$ & & & & $\begin{array}{l}\text { Almost } \\
\text { Always }\end{array}$ \\
\hline $\begin{array}{l}\text { 56. In my organization, staff are encouraged to } \\
\text { ask "why" regardless of rank. }\end{array}$ & 1 & 2 & 3 & 4 & 5 \\
\hline $\begin{array}{l}\text { 57. In my organization, staff are very } \\
\text { conscious of how changes in one part of } \\
\text { the organization can affect other parts. }\end{array}$ & 1 & 2 & 3 & 4 & 5 \\
\hline $\begin{array}{l}\text { 58. In my organization, whenever staff state } \\
\text { their views, they also ask what others } \\
\text { think. }\end{array}$ & 1 & 2 & 3 & 4 & 5 \\
\hline $\begin{array}{l}\text { 59. In my organization, staff openly discuss } \\
\text { mistakes in order to learn from them. }\end{array}$ & 1 & 2 & 3 & 4 & 5 \\
\hline $\begin{array}{l}\text { 60. When new information that would be } \\
\text { helpful to others is learned or discovered, it } \\
\text { is shared with those in the organization } \\
\text { who need it. }\end{array}$ & 1 & 2 & 3 & 4 & 5 \\
\hline $\begin{array}{l}\text { 61. In meetings at my organization, staff are } \\
\text { encouraged to discuss the values and } \\
\text { beliefs that underlie their opinions. }\end{array}$ & 1 & 2 & 3 & 4 & 5 \\
\hline $\begin{array}{l}\text { 62. In my organization, staff are resistant to } \\
\text { having their work critiqued by someone } \\
\text { else. }\end{array}$ & 1 & 2 & 3 & 4 & 5 \\
\hline
\end{tabular}




\begin{tabular}{|c|c|c|c|c|c|}
\hline $\begin{array}{l}\text { 63. In my organization, staff face conflict over } \\
\text { work issues in productive ways. }\end{array}$ & 1 & 2 & 3 & 4 & 5 \\
\hline $\begin{array}{l}\text { 64. In my organization, staff give open and } \\
\text { honest feedback to each other. }\end{array}$ & 1 & 2 & 3 & 4 & 5 \\
\hline \multicolumn{6}{|c|}{$\begin{array}{l}\text { Organizational Assessment of Progress } \\
\text { In this section, please indicate the degree to which you agree with the following statements. }\end{array}$} \\
\hline $\begin{array}{l}\text { 65. Information is gathered from stakeholders } \\
\text { to gauge how well we're doing. }\end{array}$ & $\begin{array}{c}\text { Strongly } \\
\text { Disagree }\end{array}$ & Disagree & $\begin{array}{l}\text { Neither } \\
\text { Disagree nor } \\
\text { Agree }\end{array}$ & Agree & $\begin{array}{c}\text { Strongly } \\
\text { Agree }\end{array}$ \\
\hline $\begin{array}{l}\text { 66. Currently available information tells us } \\
\text { that we need to know about the } \\
\text { effectiveness of our programs. }\end{array}$ & $\begin{array}{c}\text { Strongly } \\
\text { Disagree }\end{array}$ & Disagree & $\begin{array}{l}\text { Neither } \\
\text { Disagree nor } \\
\text { Agree }\end{array}$ & Agree & $\begin{array}{c}\text { Strongly } \\
\text { Agree }\end{array}$ \\
\hline $\begin{array}{l}\text { 67. In my organization, staff continually ask } \\
\text { themselves how they're doing, what they } \\
\text { can do better, and what is working. }\end{array}$ & $\begin{array}{l}\text { Strongly } \\
\text { Disagree }\end{array}$ & Disagree & $\begin{array}{l}\text { Neither } \\
\text { Disagree nor } \\
\text { Agree }\end{array}$ & Agree & $\begin{array}{c}\text { Strongly } \\
\text { Agree }\end{array}$ \\
\hline $\begin{array}{l}\text { 68. Evaluative activity is seen as threatening } \\
\text { the status quo. }\end{array}$ & $\begin{array}{l}\text { Strongly } \\
\text { Disagree }\end{array}$ & Disagree & $\begin{array}{l}\text { Neither } \\
\text { Disagree nor } \\
\text { Agree }\end{array}$ & Agree & $\begin{array}{c}\text { Strongly } \\
\text { Agree }\end{array}$ \\
\hline $\begin{array}{l}\text { 69. In my organization, staff are interested in } \\
\text { pursuing evaluative activity but do not } \\
\text { know how. }\end{array}$ & $\begin{array}{c}\text { Strongly } \\
\text { Disagree }\end{array}$ & Disagree & $\begin{array}{l}\text { Neither } \\
\text { Disagree nor } \\
\text { Agree }\end{array}$ & Agree & $\begin{array}{c}\text { Strongly } \\
\text { Agree }\end{array}$ \\
\hline $\begin{array}{l}\text { 70. In my organization, staff feel that } \\
\text { evaluative activity is worth the effort. }\end{array}$ & $\begin{array}{l}\text { Strongly } \\
\text { Disagree }\end{array}$ & Disagree & $\begin{array}{l}\text { Neither } \\
\text { Disagree nor } \\
\text { Agree }\end{array}$ & Agree & $\begin{array}{c}\text { Strongly } \\
\text { Agree }\end{array}$ \\
\hline $\begin{array}{l}\text { 71. Evaluation helps (or would help) us } \\
\text { provide better programs, processes, and } \\
\text { services. }\end{array}$ & $\begin{array}{c}\text { Strongly } \\
\text { Disagree }\end{array}$ & Disagree & $\begin{array}{l}\text { Neither } \\
\text { Disagree nor } \\
\text { Agree }\end{array}$ & Agree & $\begin{array}{l}\text { Strongly } \\
\text { Agree }\end{array}$ \\
\hline \multicolumn{6}{|c|}{$\begin{array}{l}\text { Relationship with Other Organizations } \\
\text { In this section, please indicate the degree to which you agree with the following statements. }\end{array}$} \\
\hline $\begin{array}{l}\text { 72. It is worthwhile to work with other } \\
\text { organizations because it allows us to } \\
\text { achieve more that we could individually as } \\
\text { an organization. }\end{array}$ & $\begin{array}{l}\text { Strongly } \\
\text { Disagree }\end{array}$ & Disagree & $\begin{array}{l}\text { Neither } \\
\text { Disagree nor } \\
\text { Agree }\end{array}$ & Agree & $\begin{array}{c}\text { Strongly } \\
\text { Agree }\end{array}$ \\
\hline $\begin{array}{l}\text { 73. When we review our programs and } \\
\text { policies, we routinely consider what other } \\
\text { organizations in our field are doing. }\end{array}$ & $\begin{array}{c}\text { Strongly } \\
\text { Disagree }\end{array}$ & Disagree & $\begin{array}{l}\text { Neither } \\
\text { Disagree nor } \\
\text { Agree }\end{array}$ & Agree & $\begin{array}{c}\text { Strongly } \\
\text { Agree }\end{array}$ \\
\hline $\begin{array}{l}\text { 74. We actively participate in a network of } \\
\text { like-minded organizations in order to } \\
\text { exchange knowledge. }\end{array}$ & $\begin{array}{c}\text { Strongly } \\
\text { Disagree }\end{array}$ & Disagree & $\begin{array}{c}\text { Neither } \\
\text { Disagree nor } \\
\text { Agree }\end{array}$ & Agree & $\begin{array}{c}\text { Strongly } \\
\text { Agree }\end{array}$ \\
\hline
\end{tabular}

\section{Additional Comments:}




\begin{tabular}{|c|c|c|c|c|c|c|c|c|c|c|}
\hline & A & $\mathrm{B}$ & $\mathrm{C}$ & $\mathrm{D}$ & $\mathrm{E}$ & $\mathrm{F}$ & G & $\mathrm{H}$ & I & $\mathrm{J}$ \\
\hline \multicolumn{11}{|l|}{ Shared Vision/ Shared Meaning } \\
\hline I really feel like my work makes a difference in people's lives. (\#2) & 3.5 & 4.2 & 4.3 & 5.0 & 4.4 & 4.6 & 3.8 & 4.7 & 4.3 & 4.4 \\
\hline $\begin{array}{l}\text { The staff I work with have very different opinions from each other about } \\
\text { where our organization needs to improve. (\#4) (reverse coded) }\end{array}$ & 3.5 & 3.6 & 3.8 & 3.3 & 3.3 & 3.9 & 2.5 & 2.4 & 1.7 & 3.0 \\
\hline $\begin{array}{l}\text { In my organization, staff understand how their work relates to the goals or } \\
\text { mission of the organization. (\#5) }\end{array}$ & 4.5 & 4.2 & 4.3 & 4.3 & 4.4 & 4.4 & 3.7 & 4.4 & 3.7 & 3.8 \\
\hline $\begin{array}{l}\text { My organizational leadership shares up to date information with staff about } \\
\text { the decision making process regarding organizational directions (\#18). }\end{array}$ & 4.5 & 4.0 & 3.0 & 4.3 & 3.9 & N/A & 3.7 & N/A & 3.0 & N/A \\
\hline \multicolumn{11}{|l|}{ Encouraging Future Scanning } \\
\hline $\begin{array}{l}\text { We are just trying to survive day to day and do not have the time to consider } \\
\text { long term plans for our organization. (\#1) (reverse coded) }\end{array}$ & 4.3 & 4.8 & 4.3 & 4.7 & 4.3 & 4.6 & 3.8 & 3.6 & 3.3 & 3.7 \\
\hline $\begin{array}{l}\text { We are actively planning in anticipation of how the external environment } \\
\text { (our client base, policy changes, etc.) may change over the next few years. } \\
(\# 3)\end{array}$ & 3.8 & 4.2 & 3.8 & 4.0 & 3.0 & 4.0 & 3.5 & 3.6 & 4.0 & 3.6 \\
\hline \multicolumn{11}{|l|}{ Inquisitiveness/ Openness } \\
\hline \multicolumn{11}{|l|}{ Ensuring Openness to New Ideas/ Diversity of thought } \\
\hline We have new ideas coming from all levels of the organization. (\#8) & 4.5 & 4.2 & 3.3 & 3.7 & 4.3 & 4.4 & 4.0 & 4.0 & 4.0 & 3.9 \\
\hline $\begin{array}{l}\text { Feedback from stakeholders in our organization often lead to changes in our } \\
\text { practices. (\#9) }\end{array}$ & 4.3 & 4.0 & 3.8 & 3.7 & 4.0 & 3.7 & 3.4 & 3.3 & 3.5 & 3.7 \\
\hline $\begin{array}{l}\text { In my organization, staff are encouraged to offer dissenting opinions and } \\
\text { alternatives. (\#11) }\end{array}$ & 5.0 & 4.6 & 4.0 & 4.0 & 4.5 & 4.4 & 3.8 & 4.1 & 4.3 & 3.9 \\
\hline
\end{tabular}




\begin{tabular}{|c|c|c|c|c|c|c|c|c|c|c|}
\hline \multicolumn{11}{|l|}{ Promoting Openness to Change } \\
\hline Being open to change is viewed positively in this organization. (\#10) & 4.5 & 4.0 & 3.8 & 4.0 & 4.3 & 4.1 & 3.4 & 3.6 & 3.7 & 3.5 \\
\hline $\begin{array}{l}\text { Most attempts to change important things are greeted with resistance. (\#15) } \\
\text { (reverse coded) }\end{array}$ & 4.5 & 4.2 & 3.5 & 3.3 & 4.1 & 4.4 & 3.5 & 3.1 & 2.7 & 3.5 \\
\hline \multicolumn{11}{|l|}{ Challenging of assumptions } \\
\hline $\begin{array}{l}\text { Any practice or assumption can be challenged, no matter whose it is, or how } \\
\text { long it has been in effect. (\#16) }\end{array}$ & 3.5 & 3.6 & 3.5 & 3.3 & 3.0 & 3.4 & 3.3 & 3.4 & 4.0 & 3.0 \\
\hline $\begin{array}{l}\text { In my organization, staff are encouraged to ask "why" regardless of rank. } \\
(\# 21)\end{array}$ & 4.8 & 4.0 & 4.0 & 4.5 & 4.0 & 4.0 & 4.2 & 4.0 & 4.5 & 3.7 \\
\hline \multicolumn{11}{|l|}{ Taking risks, Discussing mistakes } \\
\hline $\begin{array}{l}\text { In my organization, staff openly discuss mistakes in order to learn from } \\
\text { them. (\#24) }\end{array}$ & 4.5 & 4.2 & 4.3 & 4.3 & 4.6 & 3.9 & 3.3 & 4.3 & 3.7 & 2.6 \\
\hline \multicolumn{11}{|l|}{ Systems thinking/ Evaluative Thinking } \\
\hline \multicolumn{11}{|l|}{ Systems and encouragement for sharing knowledge } \\
\hline $\begin{array}{l}\text { My organization provides opportunities for staff to work together and share } \\
\text { knowledge. (\#12) }\end{array}$ & 4.8 & 4.6 & 4.3 & 4.3 & 4.4 & 4.3 & 3.7 & 4.1 & 4.0 & 4.1 \\
\hline $\begin{array}{l}\text { When new information that would be helpful to others is learned or } \\
\text { discovered, it is shared with those in the organization who need it. (\#25) }\end{array}$ & 4.3 & 3.8 & 3.3 & 4.5 & 4.4 & 3.6 & 4.4 & 3.3 & 4.0 & 3.5 \\
\hline \multicolumn{11}{|l|}{ Interrelatedness of Organization } \\
\hline $\begin{array}{l}\text { In my organization, staff are very conscious of how changes in one part of } \\
\text { the organization can affect other parts. }(\# 22)\end{array}$ & 4.3 & 4.5 & 3.8 & 4.0 & 3.5 & 4.1 & 3.7 & 4.0 & 4.0 & 3.1 \\
\hline \multicolumn{11}{|l|}{ Evaluative Attitude } \\
\hline $\begin{array}{l}\text { Information is gathered from stakeholders to gauge how well we're doing. } \\
(\# 32)\end{array}$ & 3.8 & 3.8 & 3.3 & 4.3 & 4.3 & 3.7 & 2.9 & 3.1 & 4.0 & 3.0 \\
\hline $\begin{array}{l}\text { Currently available information tells us that we need to know about the } \\
\text { effectiveness of our programs. (\#33) }\end{array}$ & 4.0 & 4.0 & 3.8 & 4.7 & 4.0 & 3.9 & 3.6 & 3.7 & 4.0 & 3.6 \\
\hline
\end{tabular}




\begin{tabular}{|c|c|c|c|c|c|c|c|c|c|c|}
\hline $\begin{array}{l}\text { In my organization, staff continually ask themselves how they're doing, what } \\
\text { they can do better, and what is working. (\#34) }\end{array}$ & 4.3 & 4.2 & 4.0 & 4.3 & 4.3 & 3.7 & 3.6 & 3.4 & 3.7 & 3.3 \\
\hline $\begin{array}{l}\text { Evaluative activity is seen as threatening the status quo. (\#35) (reverse } \\
\text { coded) }\end{array}$ & 4.3 & 4.2 & 4.5 & 4.3 & 4.0 & 3.7 & 3.8 & 3.4 & 3.7 & 2.9 \\
\hline $\begin{array}{l}\text { My organization's leadership uses data/information to inform their decision- } \\
\text { making. (\#17) }\end{array}$ & 4.3 & 3.8 & 3.8 & 3.7 & 4.1 & N/A & 3.8 & N/A & 4.0 & N/A \\
\hline \multicolumn{11}{|l|}{ A Community of Learners: Informal Learning/ Social Capital } \\
\hline \multicolumn{11}{|l|}{ Promoting Dialogue } \\
\hline We have fruitful, constructive debates about new ideas. (\#14) & 4.3 & 4.2 & 4.3 & 3.7 & 4.0 & 4.3 & 3.3 & 3.6 & 3.7 & 3.6 \\
\hline $\begin{array}{l}\text { In my organization, whenever staff state their views, they also ask what } \\
\text { others think.(\#23) }\end{array}$ & 4.5 & 5.0 & 4.3 & 4.3 & 4.4 & 4.1 & 4.3 & 3.9 & 4.3 & 3.5 \\
\hline $\begin{array}{l}\text { In meetings at my organization, staff are encouraged to discuss the values } \\
\text { and beliefs that underlie their opinions. (\#26) }\end{array}$ & 3.5 & 4.6 & 3.0 & 4.3 & 4.6 & 4.3 & 3.8 & 3.6 & 3.7 & 3.3 \\
\hline \multicolumn{11}{|l|}{ Providing Critical Feedback } \\
\hline $\begin{array}{l}\text { In my organization, staff are resistant to having their work critiqued by } \\
\text { someone else. (\#27) (reverse coded) }\end{array}$ & 4.5 & 3.6 & 3.3 & 5.0 & 4.0 & 4.1 & 2.8 & 3.6 & 3.0 & 3.4 \\
\hline $\begin{array}{l}\text { My organization's leadership is open to constructive, critical feedback from } \\
\text { the staff. (\#29) }\end{array}$ & 4.5 & 4.4 & 3.3 & 5.0 & 4.4 & N/A & 3.9 & N/A & 4.0 & N/A \\
\hline \multicolumn{11}{|l|}{ Enhancing Cooperation } \\
\hline $\begin{array}{l}\text { In my organization, staff face conflict over work issues in productive ways } \\
(\# 28) \text {. }\end{array}$ & 4.0 & 3.8 & 2.8 & 4.0 & 4.0 & 3.7 & 3.4 & 4.0 & 3.0 & 2.8 \\
\hline We tend to work collaboratively with each other. (\#13) & 4.8 & 4.2 & 4.5 & 4.0 & 4.3 & 4.3 & 3.7 & 3.9 & 3.7 & 3.6 \\
\hline \multicolumn{11}{|l|}{ External Focus/ Network Connectedness } \\
\hline $\begin{array}{l}\text { We are actively planning in anticipation of how the external environment } \\
\text { (our client base, policy changes, etc.) may change over the next few years. }\end{array}$ & 3.8 & 4.2 & 3.8 & 4.0 & 3.0 & 4 & 3.5 & 3.6 & 4.0 & 3.6 \\
\hline
\end{tabular}




\begin{tabular}{|l|l|l|l|l|l|l|l|l|l|l|}
\hline (\#3 ) (counted twice) & & & & & & & \\
\hline $\begin{array}{l}\text { Feedback from stakeholders in our organization often lead to changes in our } \\
\text { practices. (\#9) (counted twice) }\end{array}$ & 4.3 & 4.0 & 3.8 & 3.7 & 4.0 & 3.7 & 3.4 & 3.3 & 3.5 & 3.7 \\
\hline $\begin{array}{l}\text { Information is gathered from stakeholders to gauge how well we're doing. } \\
\text { (\#32) (counted twice) }\end{array}$ & 3.8 & 3.8 & 3.3 & 4.3 & 4.3 & 3.7 & 2.9 & 3.1 & 4.0 & 3.0 \\
\hline $\begin{array}{l}\text { It is worthwhile to work with other organizations because it allows us to } \\
\text { achieve more that we could individually as an organization. (\#37) }\end{array}$ & 4.0 & 4.6 & 3.5 & 4.0 & 3.8 & 4.1 & 4.3 & 4.7 & 4.7 & 3.7 \\
\hline $\begin{array}{l}\text { When we review our programs and policies, we routinely consider what } \\
\text { other organizations in our field are doing. (\#38) }\end{array}$ & 4.0 & 4.0 & 3.5 & 2.5 & 2.5 & 4.0 & 2.7 & 3.6 & 3.7 & 3.6 \\
\hline $\begin{array}{l}\text { We actively participate in a network of like-minded organizations in order to } \\
\text { exchange knowledge. (\#39) }\end{array}$ & 4.8 & 4.0 & 3.8 & 4.0 & 3.6 & 4.0 & 3.6 & 4.0 & 3.7 & 4.0 \\
\hline
\end{tabular}




\section{APPENDIX H: DESCRIPTIVE STATISTICS BY ORGANIZATION}

Organization \# A

Descriptive Statistics

\begin{tabular}{|l|r|r|r|}
\hline & $\mathrm{N}$ & Mean & Std. Deviation \\
\hline a1 & 11 & 3.82 & 1.168 \\
a2 & 11 & 3.82 & .751 \\
a3 & 11 & 3.45 & 1.128 \\
a4 & 11 & 2.45 & 1.214 \\
a5 & 11 & 3.73 & 1.191 \\
b8 & 11 & 4.00 & .775 \\
b9 & 11 & 3.36 & 1.027 \\
b10 & 11 & 3.36 & .924 \\
b11 & 11 & 3.82 & .751 \\
b12 & 11 & 3.73 & .905 \\
b13 & 11 & 3.73 & 1.009 \\
b14 & 11 & 3.27 & 1.104 \\
b15 & 11 & 3.45 & .934 \\
b16 & 11 & 3.27 & 1.191 \\
b17 & 11 & 3.82 & .874 \\
b18 & 11 & 3.73 & 1.104 \\
c21 & 11 & 4.18 & .982 \\
c22 & 10 & 3.70 & 1.059 \\
c23 & 11 & 4.27 & .905 \\
c24 & 11 & 3.27 & 1.104 \\
c25 & 11 & 4.36 & .809 \\
c26 & 11 & 3.82 & .982 \\
c27 & 11 & 2.82 & 1.250 \\
c28 & 11 & 3.36 & .809 \\
c29 & 11 & 3.91 & .831 \\
c32 & 11 & 2.91 & .944 \\
c33 & 11 & 3.64 & .809 \\
c34 & 11 & 3.64 & 1.286 \\
c35 & 10 & 3.80 & 1.033 \\
d37 & 10 & 4.30 & .483 \\
d38 & 10 & 2.70 & .675 \\
d39 & 3.60 & 1.265 \\
Valid N (listwise) & & \\
\hline & & & \\
\hline
\end{tabular}


Organization \# B

\section{Descriptive Statistics}

\begin{tabular}{|l|r|r|r|}
\hline & $N$ & Mean & Std. Deviation \\
\hline a1 & 5 & 4.80 & .447 \\
a2 & 5 & 4.20 & .447 \\
a3 & 5 & 4.20 & .447 \\
a4 & 5 & 3.60 & .894 \\
a5 & 5 & 4.20 & .447 \\
b8 & 5 & 4.20 & .447 \\
b9 & 5 & 4.00 & .000 \\
b10 & 5 & 4.00 & .000 \\
b11 & 5 & 4.60 & .548 \\
b12 & 5 & 4.60 & .548 \\
b13 & 5 & 4.20 & .837 \\
b14 & 5 & 4.20 & .447 \\
b15 & 5 & 4.20 & .837 \\
b16 & 5 & 3.60 & .894 \\
b17 & 5 & 3.80 & .447 \\
b18 & 5 & 4.00 & .707 \\
c21 & 5 & 4.00 & .707 \\
c22 & 4 & 4.50 & .577 \\
c23 & 5 & 5.00 & .000 \\
c24 & 5 & 1.304 \\
c25 & 5 & .447 \\
c26 & 5 & .548 \\
c27 & 520 & .894 \\
c28 & 5 & .447 \\
c29 & 5 & .548 \\
c32 & 5 & .095 \\
c33 & 5 & .707 \\
c34 & 5 & .837 \\
c35 & 5 & 4.80 \\
d37 & 5 & .548 \\
d38 & 5 & .707 \\
d39 & 5.60 & 1.414 \\
Valid N (listwise) & 5.60 & \\
\hline & 5 & 3.80 & \\
\hline
\end{tabular}


Organization \#C

\section{Descriptive Statistics}

\begin{tabular}{|c|c|c|c|}
\hline & $\mathrm{N}$ & Mean & Std. Deviation \\
\hline a1 & 4 & 4.25 & .500 \\
\hline a2 & 4 & 4.25 & .500 \\
\hline a3 & 4 & 3.75 & 1.258 \\
\hline a4 & 4 & 3.75 & .957 \\
\hline a5 & 4 & 4.25 & .957 \\
\hline b8 & 4 & 3.25 & 1.500 \\
\hline b9 & 4 & 3.75 & .500 \\
\hline b10 & 4 & 3.75 & .500 \\
\hline b11 & 4 & 4.00 & .816 \\
\hline b12 & 4 & 4.25 & .500 \\
\hline b13 & 4 & 4.50 & .577 \\
\hline b14 & 4 & 4.25 & .957 \\
\hline b15 & 4 & 3.50 & 1.291 \\
\hline b16 & 4 & 3.50 & 1.000 \\
\hline b17 & 4 & 3.75 & .500 \\
\hline b18 & 4 & 3.00 & 1.155 \\
\hline c21 & 4 & 4.00 & 1.155 \\
\hline c22 & 4 & 3.75 & .957 \\
\hline $\mathrm{c} 23$ & 4 & 4.25 & .500 \\
\hline c24 & 4 & 4.25 & .957 \\
\hline c25 & 4 & 3.25 & .957 \\
\hline c26 & 4 & 3.00 & 1.414 \\
\hline c27 & 4 & 3.25 & .957 \\
\hline c28 & 4 & 2.75 & 1.258 \\
\hline c29 & 4 & 3.25 & 1.708 \\
\hline c32 & 4 & 3.25 & .500 \\
\hline c33 & 4 & 3.75 & .957 \\
\hline c34 & 4 & 4.00 & .816 \\
\hline c35 & 4 & 4.50 & .577 \\
\hline d37 & 4 & 3.50 & 1.000 \\
\hline d38 & 4 & 3.50 & 1.732 \\
\hline d39 & 4 & 3.75 & .957 \\
\hline Valid N (listwise) & 4 & & \\
\hline
\end{tabular}


Organization \#D

\section{Descriptive Statistics}

\begin{tabular}{|c|c|c|c|}
\hline & $\mathrm{N}$ & Mean & Std. Deviation \\
\hline a1 & 4 & 4.25 & .957 \\
\hline a2 & 4 & 3.50 & 1.000 \\
\hline a3 & 4 & 3.75 & .500 \\
\hline a4 & 4 & 3.50 & 1.291 \\
\hline a5 & 4 & 4.50 & .577 \\
\hline b8 & 4 & 4.50 & 1.000 \\
\hline b9 & 4 & 4.25 & .957 \\
\hline b10 & 4 & 4.50 & 1.000 \\
\hline b11 & 4 & 5.00 & .000 \\
\hline b12 & 4 & 4.75 & .500 \\
\hline b13 & 4 & 4.75 & .500 \\
\hline b14 & 4 & 4.25 & 1.500 \\
\hline b15 & 4 & 4.50 & .577 \\
\hline b16 & 4 & 3.50 & 1.000 \\
\hline b17 & 4 & 4.25 & .957 \\
\hline b18 & 4 & 4.50 & .577 \\
\hline c21 & 4 & 4.75 & .500 \\
\hline c22 & 4 & 4.25 & .957 \\
\hline c23 & 4 & 4.50 & .577 \\
\hline c24 & 4 & 4.50 & 1.000 \\
\hline c25 & 4 & 4.25 & .500 \\
\hline c26 & 4 & 3.50 & 1.732 \\
\hline c27 & 4 & 4.50 & .577 \\
\hline c28 & 4 & 4.00 & .816 \\
\hline c29 & 4 & 4.50 & 1.000 \\
\hline c32 & 4 & 3.75 & .500 \\
\hline c33 & 4 & 4.00 & .000 \\
\hline c34 & 4 & 4.25 & .500 \\
\hline c35 & 4 & 4.25 & .957 \\
\hline d37 & 4 & 4.00 & 1.414 \\
\hline d38 & 4 & 4.00 & .816 \\
\hline d39 & 4 & 4.75 & .500 \\
\hline Valid N (listwise) & 4 & & \\
\hline
\end{tabular}




\begin{tabular}{|l|r|r|r|}
\hline & $\mathrm{N}$ & \multicolumn{1}{|c|}{ Mean } & Std. Deviation \\
\hline N1 & 7 & 3.57 & .787 \\
N2 & 7 & 4.71 & .488 \\
N3 & 7 & 3.57 & 1.134 \\
N4 & 7 & 2.43 & .976 \\
N5 & 7 & 4.43 & .787 \\
N7 & 7 & 4.00 & 1.000 \\
N8 & 7 & 3.29 & 1.380 \\
N9 & 7 & 3.57 & 1.272 \\
N10 & 7 & 4.14 & 1.069 \\
N11 & 7 & 4.14 & 1.069 \\
N12 & 7 & 3.86 & 1.069 \\
N13 & 7 & 3.57 & .787 \\
N14 & 7 & 3.14 & 1.069 \\
N15 & 7 & 3.43 & .976 \\
N16 & 7 & 4.00 & 1.155 \\
N17 & 7 & 4.00 & 1.000 \\
N18 & 7 & 3.86 & .900 \\
N19 & 7 & 4.29 & 1.113 \\
N20 & 7 & 3.29 & .951 \\
N21 & 7 & 3.57 & 1.134 \\
N22 & 7 & 3.57 & 1.272 \\
N23 & 7 & 4.00 & .577 \\
N24 & 7 & 3.71 & .951 \\
N25 & 7 & 3.14 & .900 \\
N26 & 7 & 3.71 & .756 \\
N27 & 7 & 3.43 & .976 \\
N28 & 7 & 3.43 & 1.134 \\
N29 & 7.29 & .488 \\
N30 & 7 & 3.71 & .756 \\
N31 & 7.86 & .488 \\
N32 & 7.069 \\
N33 & 7.00 \\
N34 & 7.00 \\
Valid N (listwise) & & 1.155 \\
\hline
\end{tabular}




\section{Descriptive Statistics}

\begin{tabular}{|l|r|r|r|}
\hline & $\mathrm{N}$ & \multicolumn{1}{|c|}{ Mean } & Std. Deviation \\
\hline N1 & 15 & 3.73 & 1.223 \\
N2 & 15 & 4.40 & .737 \\
N3 & 15 & 3.60 & 1.056 \\
N4 & 15 & 3.00 & 1.069 \\
N5 & 15 & 3.80 & .775 \\
N7 & 15 & 3.93 & .884 \\
N8 & 15 & 3.73 & 1.033 \\
N9 & 15 & 3.53 & 1.060 \\
N10 & 15 & 3.87 & .915 \\
N11 & 15 & 4.07 & .704 \\
N12 & 15 & 3.60 & .828 \\
N13 & 15 & 3.60 & .828 \\
N14 & 15 & 3.53 & .915 \\
N15 & 15 & 3.00 & 1.363 \\
N16 & 15 & 3.67 & 1.234 \\
N17 & 15 & 3.13 & 1.125 \\
N18 & 15 & 3.47 & .915 \\
N19 & 15 & 2.60 & .828 \\
N20 & 15 & 3.53 & .990 \\
N21 & 15 & 3.27 & 1.163 \\
N22 & 15 & 3.40 & .986 \\
N23 & 15 & 2.80 & .676 \\
N24 & 15 & 3.07 & .961 \\
N25 & 15 & 3.00 & 1.134 \\
N26 & 15 & 3.60 & .986 \\
N27 & 15 & 3.27 & 1.033 \\
N28 & 15 & 2.93 & 1.100 \\
N29 & 15 & 3.67 & .724 \\
N30 & 15 & 3.73 & .594 \\
N31 & & 3.47 & .834 \\
N32 & 3.73 & 1.163 \\
N33 & 3.60 & 1.183 \\
N34 & 4.00 & .535 \\
Valid N (listwise) & & \\
\hline
\end{tabular}




\section{Descriptive Statistics}

\begin{tabular}{|l|r|r|r|}
\hline & $N$ & Mean & Std. Deviation \\
\hline n1 & 7 & 4.57 & .535 \\
n2 & 7 & 4.00 & .816 \\
n3 & 7 & 4.57 & .535 \\
n4 & 7 & 3.86 & .900 \\
n5 & 7 & 4.43 & .535 \\
n6 & 7 & 4.43 & .535 \\
n7 & 7 & 3.71 & .488 \\
n8 & 7 & 4.14 & .690 \\
n9 & 7 & 4.43 & .535 \\
n10 & 7 & 4.14 & .378 \\
n11 & 7 & 4.00 & .577 \\
n12 & 7 & 4.29 & .488 \\
n13 & 7 & 4.43 & .535 \\
n14 & 7 & 3.43 & .535 \\
n15 & 7 & 4.29 & .756 \\
n16 & 7 & 4.14 & .690 \\
n17 & 7 & 4.29 & .488 \\
n18 & 7 & 3.86 & .690 \\
n19 & 7 & 3.57 & .535 \\
n20 & 7 & 4.29 & .756 \\
n21 & 7 & 4.14 & .378 \\
n22 & 7 & 3.86 & .690 \\
n23 & 7 & 3.86 & .378 \\
n24 & 7 & 3.71 & .488 \\
n25 & 7 & 3.86 & .378 \\
n26 & 7 & .951 \\
n27 & 7 & .756 \\
n28 & 7.71 & .756 \\
n29 & 7 & .816 \\
n30 & 4.71 & .787 \\
n31 & 7.00 & .378 \\
n32 & 4.43 & .577 \\
n33 & 7.000 \\
Valid N (listwise) & & \\
\hline & 7 & & \\
\hline
\end{tabular}


Organization \#H

\section{Descriptive Statistics}

\begin{tabular}{|l|r|r|r|}
\hline & $\mathrm{N}$ & \multicolumn{1}{|c|}{ Mean } & Std. Deviation \\
\hline a1 & 3 & 3.33 & 1.155 \\
a2 & 3 & 4.33 & .577 \\
a3 & 3 & 4.00 & .000 \\
a4 & 3 & 1.67 & .577 \\
a5 & 3 & 3.67 & .577 \\
b8 & 2 & 4.00 & .000 \\
b9 & 2 & 3.50 & .707 \\
b10 & 3 & 3.67 & .577 \\
b11 & 3 & 4.33 & .577 \\
b12 & 3 & 4.00 & .000 \\
b13 & 3 & 3.67 & .577 \\
b14 & 3 & 3.67 & .577 \\
b15 & 3 & 2.67 & .577 \\
b16 & 3 & 4.00 & .000 \\
b17 & 2 & 4.00 & .000 \\
b18 & 2 & 3.00 & 1.414 \\
c21 & 2 & 4.50 & .707 \\
c22 & 3 & 4.00 & 1.000 \\
c23 & 3 & 4.33 & .577 \\
c24 & 3 & .577 \\
c25 & 3 & 1.000 \\
c26 & 3 & .577 \\
c27 & 3 & .000 \\
c28 & 3 & 1.000 \\
c29 & 3 & .000 \\
c32 & 3 & .000 \\
c33 & 3 & .000 \\
c34 & 3 & .577 \\
c35 & 3 & .577 \\
d37 & 3.60 & .577 \\
d38 & 3.67 & .577 \\
d39 & 3.00 & .577 \\
Valid N (listwise) & 3.00 & \\
\hline & 3 & 3.67 & \\
\hline
\end{tabular}


Organization \# I

\section{Descriptive Statistics}

\begin{tabular}{|l|r|r|r|}
\hline & $\mathrm{N}$ & \multicolumn{1}{|c|}{ Mean } & Std. Deviation \\
\hline a1 & 4 & 4.67 & .577 \\
a2 & 4 & 5.00 & .000 \\
a3 & 4 & 4.00 & .000 \\
a4 & 4 & 3.33 & .577 \\
a5 & 4 & 4.33 & .577 \\
b8 & 4 & 3.67 & 1.155 \\
b9 & 4 & 3.67 & 1.528 \\
b10 & 4 & 4.00 & 1.000 \\
b11 & 4 & 4.00 & 1.000 \\
b12 & 4 & 4.33 & 1.155 \\
b13 & 4 & 4.00 & 1.000 \\
b14 & 4 & 3.67 & 1.528 \\
b15 & 4 & 3.33 & 1.528 \\
b16 & 4 & 3.33 & 1.528 \\
b17 & 4 & 3.67 & 1.155 \\
b18 & 4 & 4.33 & 1.155 \\
c21 & 3 & 4.50 & .707 \\
c22 & 3 & 4.00 & .000 \\
c23 & 4 & 4.33 & 1.155 \\
c24 & 4 & .577 \\
c25 & 4.33 & .707 \\
c26 & 4 & .577 \\
c27 & 4 & .000 \\
c28 & 4 & 1.000 \\
c29 & 4 & .000 \\
c32 & 4 & .157 \\
c33 & 4 & .577 \\
c34 & 4 & 4.35 \\
c35 & 4 & 4.33 \\
d37 & 4.000 \\
d38 & 4 & .707 \\
d39 & 4.00 & .000 \\
Valid N (listwise) & 4.00 & \\
\hline & & & \\
\hline
\end{tabular}




\section{Organization \# $\mathbf{J}$}

\section{Descriptive Statistics}

\begin{tabular}{|c|c|c|c|}
\hline & $\mathrm{N}$ & Mean & Std. Deviation \\
\hline a1 & 8 & 4.25 & 1.035 \\
\hline a2 & 8 & 4.38 & 1.061 \\
\hline a3 & 8 & 3.00 & 1.069 \\
\hline a4 & 8 & 3.25 & 1.165 \\
\hline a5 & 8 & 4.38 & .518 \\
\hline b8 & 8 & 4.25 & .463 \\
\hline b9 & 8 & 4.00 & .926 \\
\hline b10 & 8 & 4.25 & .707 \\
\hline b11 & 8 & 4.50 & .535 \\
\hline b12 & 8 & 4.37 & .744 \\
\hline b13 & 8 & 4.25 & .886 \\
\hline b14 & 8 & 4.00 & 1.069 \\
\hline b15 & 8 & 4.13 & .354 \\
\hline b16 & 8 & 3.00 & 1.195 \\
\hline b17 & 8 & 4.13 & .641 \\
\hline b18 & 8 & 3.88 & .641 \\
\hline c21 & 8 & 4.00 & .756 \\
\hline c22 & 8 & 3.50 & .926 \\
\hline c23 & 7 & 4.43 & .535 \\
\hline c24 & 8 & 4.63 & .518 \\
\hline c25 & 8 & 4.38 & .744 \\
\hline c26 & 8 & 4.63 & .518 \\
\hline c27 & 8 & 4.00 & .535 \\
\hline c28 & 8 & 4.00 & .756 \\
\hline c29 & 8 & 4.38 & .518 \\
\hline c32 & 8 & 4.25 & .463 \\
\hline c33 & 8 & 4.00 & .756 \\
\hline c34 & 8 & 4.25 & .463 \\
\hline c35 & 8 & 4.00 & .926 \\
\hline d37 & 8 & 3.75 & .707 \\
\hline d38 & 8 & 2.50 & 1.069 \\
\hline d39 & 7 & 3.57 & .976 \\
\hline Valid N (listwise) & 7 & & \\
\hline
\end{tabular}




\section{BIBLIOGRAPHY}

Alterman, R. and Vraneski, A. (1995). "Who's not Afraid of Public Participation?" City and Region. 23: 123

Anderson, W. T. (1990). Reality Isn't What It Used to Be. San Francisco, CA: Harper and Row.

Argyris, C. (1998). "Teaching Smart People How to Learn” In Harvard Business Review on Knowledge Management. Boston, MA: Harvard Business School Publishing. p. 81-108.

Argyris, C. (2001) "Good Communication that Blocks Learning.” In Harvard Business Review on Organizational Learning. Boston, MA: Harvard Business School Publishing. P. 87-109.

Argyris, C., \& Schon, D. (1978) Theory in Practice: Increasing Professional Effectiveness. San Francisco: Jossey-Bass.

Argyris, C., \& Schon, D. (1996) Organizational learning II: Theory, method, and practice. Reading, MA: Addison-Wesley.

Asa, L. (2005) Personal Interview with the Researcher, May 22 ${ }^{\text {nd }}, 2005$.

Ben Elia, N. (1993). "Policymaking and Management in Israeli Local Government: Evolving Trends and Strategic Challenges." Policy Studies Journal. 21, (1): 115-125.

Blumenthal, B. (2003). Investing in Capacity Building: A Guide to High-Impact Approaches. New York: The Foundation Center.

Bickel, W. E., Millett, R., and Nelson, C. A. (February, 2002). "Challenges to the role of evaluation in supporting organizational learning in foundations," (www.foundationnews.org/webextra/learning web.htm). Washington, D. C.: Council on Foundations.

Bohn, R. (2001) "Stop Fighting Fires”. In Harvard Business Review on Organizational Learning. Boston, MA: Harvard Business School Publishing. 161- 183.

Botcheva, L, White, C.R. and Huffman, L. (2002) "Learning Cultures and Outcomes Measurement Practices in Community Agencies". The American Journal of Evaluation. (23) (4) 421-434.

Campobasso, L. and Davis,D. (2001) Reflections on Capacity Building. Woodland Hills, CA: California Wellness Foundation. (http://www.tcwf.org/reflections/2001/april/)

Chelimsky, E. (2001)"What Evaluation Could Do To Support Foundations." The American Journal of Evaluation. (22) (1). 13-28.

Chen, H.T. (1990). Theory-Driven Evaluations. Thousand Oak, CA: Sage.

Christensen, C and Overdorf, M. (2001). Meeting the Challenge of Disruptive Change". ." In Harvard Business Review on Innovation. Boston, MA: Harvard Business School Publishing. P. 103-130. 
Cnaan, R. A. (1987). “The Evolution of Israel's Welfare State.” In R. R. Friedmann, N. Gilbert and J. Sherer (eds.) Modern Welfare States. Brighton, England: Weathsheaf: 174-207.

Cohen, D. and Prusak, L. (2001). In Good Company: How Social Capital Makes Organizations Work. Boston, MA: Harvard Business School Press.

Collins, J and Porras, J. (1997) Built to Last: Successful Habit of Visionary Companies. New York: Harper Business

Collins, J. (2001) Good to Great: Why Some Companies Make the Leap and Others Don't. New York: Harper Business

Connolly, P. and York, P. (2003). Building the Capacity of Capacity Builders to Last: A Grantmaker's Guide to Strengthening Nonprofit Organizations. Philadelphia, PA : The Conservation Company. (http://www.tccgrp.com/ pdfs/buildingthecapacityofcapacitybuilders.pdf)

Cooperider, D. L. and Srivastva, S. (1987). “Appreciative Inquiry in Organizational Life.” Research in Organizational Change and Development, 1, 129-169.

Cousins, J.B. and Earl, L. (1995). Participatory Evaluation in Education. Washington, D.C.: Falmer Press

Cousins, J.B. and Whitmore, E. (1998) "Framing Participatory Evaluation". In E. Whitmore (ed.) New Directions for Evaluation. 80: Winter 1998, San Francisco: Jossey-Bass Publishers.

Coutu, D. 2002. Interview with Schein, The Anxiety of Learning. Reprint R0203H, March 2002. Boston, MA: Harvard Business School Press. p. 100-106.

Davenport, T and Prusak , L. (2000) Working Knowledge: How Organizations Manage What They Know. Boston, MA: Harvard Business School Press.

David, T. (2002) Reflections on Sustainability. Woodland Hills, CA: California Wellness Foundation. (http://www.tcwf.org/reflections/2002/feb/index.htm)

Davidson, J. E. (2001). The Meta-Learning Organization: A Model and Methodology for Evaluating Organizational Learning Capacity. (Doctoral dissertation, available from UMI Microform, \#3015945).

Donaldson, S.I. and Scriven, M. (2003). "Diverse Visions for Evaluation in the New Millennium: Should We Integrate or Embrace Diversity?" In S.I. Donaldson and M. Scriven (Eds), Evaluating Social Programs and Problems: Visions for the New Millennium. (pp. 3-18). London: Lawrence Erlbaum Associates

Doron, A. (1985). “The Israeli Welfare State at Crossroads.” Journal of Social Policy. 14, (4): 513-526.

Eisenstadt, S.N. (1985). "The Israeli Political System and the Transformation of the Israeli Society." In E. Krausz (ed.), Politics and Society in Israel. New Brunswick, N.J. : Transaction Books.

Eoyang, G. and Berkas, T. (1998). Evaluation in a Complex Adaptive System. (http://www.winternet.com/ eoyang/Complexity_Intro.htm) 
Fetterman, D.(2001) Foundations of Empowerment Evaluation. Thousand Oaks, California: Sage Publications.

Fine, A., Kopf, N. and Thayer, C. (2001). Echoes from the Field: Proven Capacity-Building Principles for Nonprofits. Washington, D.C. Environmental Support Center. (http://www.envsc.org/bestpractices.pdf)

Firstenberg, P. B. (2003) Philanthropy's Challenge: Building Nonprofit Capacity Through Venture Grantmaking. New York: The Foundation Center.

Fukuyama, F. (1995) The Social Virtues and the Creation of Prosperity. London: Penguin

Gantz McKay (2005). Personal Interview with Researcher. June $12^{\text {th }}, 2005$

Garvin, D. (1998) “Building a Learning Organization”. In Harvard Business Review on Knowledge Management. Boston, MA: Harvard Business School Publishing. P. 47-80

Garvin, D. (2000) Learning in action: A guide to putting the learning organization to work. Cambridge, MA: Harvard Business School Press.

Gidron, B. (1992). “A Resurgent Third Sector and its Relationship to Government in Israel." In Gidron, B. et. al. Government and the Third Sector. San Francisco: Jossey -Bass: 176-195

Gidron, B. and Katz, H. (1998) Defining the Nonprofit Sector: Israel. Working paper No. 26, Baltimore, MD: Johns Hopkins University

Gray and Associates. (1998) Evaluation with Power. Washington, D. C.: Independent Sector.

Greene, J. C. (1998). "Qualitative Program Evaluation: Practice and Promise”. In N. Denzin and Y. Lincoln (Eds), Collecting and Interpreting Qualitative Materials. Thousand Oaks, CA: Sage. Publications. p. 372-399.

Hernandez, G. and Visher, M. G. (2001) Creating a Culture of Inquiry: Changing Methods- and Mindson the Use of Evaluation in Nonprofit Organizations. San Francisco: James Irvine Foundation. (http://www.irvine.org/pdfs/Creating_a_Culture.pdf)

Heuer, M. (1999). Nonprofit Organizational Effectiveness: A Review of the Literature. Washington, D.C.: Innovation Network. (http://www.innonet.org/resources/lcplitReview.pdf)

House, E. (2001). "Responsive Evaluation (and Its Influence on Deliberative Democratic Evaluation)." New Directions For Evaluation, 92: 23-30.

Katz, O. (2000). Nonprofit Legal Guide. Published by Shatil. Jerusalem, Israel: New Israel Fund ( in Hebrew).

Kiesler, S. and Sproull, L. (1982). “Managerial Response to Changing Environments.” Administrative Science Quarterly. Vol. 27: 559

King, J. (1995) "Involving Practitioners in Evaluation Studies." In Cousins, J.B. and Earl, L. (Eds.) Participatory Evaluation in Education. Washington ,D.C.: Falmer Press 
Kirkhart, K. (2000). " Reconceptualizing Evaluating Use: An Integrated Theory of Influence.” In V. Caracelli and H. Preskill (eds.) The Expanding Scope of Evaluation Use. New Directions for Evaluation, no. 88. San Francisco: Jossey-Bass, 2000 (55-70).

Kleiner, A. and Roth, G. (1998) "How to Make Experience Your Company's Best Teacher" In Harvard Business Review on Knowledge Management. Boston, MA: Harvard Business School Publishing. p. $137-152$.

Kuhn, T. (1996). The Structure of Scientific Revolutions. 3rd Edition. Chicago and London: The University of Chicago Press.

Lafleur, C. (1995) “A Participatory Approach to District-Level Program Evaluation.” In Cousins, J.B. and Earl, L. (Eds.) Participatory Evaluation in Education. Washington, D.C.: Falmer Press

Lazin, F. (1996). “Citizen Participation: Project Renewal in Israel.” Journal of Urban Affairs. 18,(3):307-321.

Letts, C.W., Ryan, W. and Grossman, A. (1999). High Performance Nonprofit Organizations: Managing Upstream for Greater Impact. New York: John Wiley and Sons, Inc.

Light, P. (2004) Sustaining Nonprofit Performance. Washington, DC: Brookings Institution Press.

Light, P. Hubbard, E. T., and Kibbe, B. (2004). The Capacity Building Challenge. New York: The Foundation Center. (http://fdncenter.org/for_grantmakers/practice_matters/practicematters_07_part2.pdf)

Marsick, V., Volpe, M. and Watkins, K. (2003) Demonstrating the Value of an Organization's Learning Culture: the Dimensions of the Learning Organization Questionnaire.” In Watkins, K and Marsick, V. (Eds.) Advances in Developing Human Resources, 5 (2) p. 132-151.

Marsick, V., Volpe, M. and Watkins, K. (1999) Theory and Practice of Informal Learning in the Knowledge Era. In Informal Learning on the Job Volpe, M. and Marsick, V. (Eds.) Advances in Developing Human Resources, 3, p. 80- 95.

McHargue, S. (2003) "Learning for Performance in Nonprofit Organizations." In Watkins, K and Marsick, V. (Eds.) Advances in Developing Human Resources, 5 (2) p. 196-204.

McKinsey and Company, (2001) Effective Capacity Building in Nonprofit Organizations. Reston, VA: Venture Philanthropy Partners. (http://www.venturephilanthropypartners.org/usr_doc/full_rpt.pdf)

McPhee, P. and Bare, J. (2001) "Introduction." In De Vita, C. J. and C. F. (Eds.) Building Capacity in Nonprofit Organizations. Washington, D.C.: Urban Institute, p.1- 5. (http://www.urban.org/pdfs/building_capacity.pdf)

Monaghan, J. and Just, P. (2000). Social and Cultural Anthropology. Oxford; Oxford University Press.

O'Neil, J. (2003) "Participant's Guide for Interpreting Results on the Dimensions of the Learning Organization Questionnaire." In Watkins, K and Marsick, V. (Eds.) Advances in Developing Human Resources, 5 (2) Appendix A. 
Patton, M.Q. (1980). Qualitative Evaluation Methods. Beverly Hills, CA: Sage.

Patton, M.Q. (1997). Utilization- Focused Evaluation. 3rd Ed. Thousand Oaks, CA: Sage Publications.

Patton, M.Q. (1998). Cited in Gray and Associates. (1998) Evaluation with Power. Washington, D. C.: Independent Sector.

Patton, M.Q. (2002). Qualitative Evaluation Methods, Third Edition. Beverly Hills, CA: Sage.

Porter, M. and Kramer, M. (1999). "Philanthropy's New Agenda: Creating Value." Harvard Business Review, Nov.-Dec.

Preskill, H and Torres, R. (2000)a. “The Learning Dimension of Evaluation Use.” In V. Caracelli and H. Preskill (eds.) The Expanding Scope of Evaluation Use. New Directions for Evaluation, no. 88. San Francisco: Jossey-Bass, 2000 (55-70).

Preskill, H and Torres, R. (2000) b. "The Readiness for Organizational Learning and Evaluation Instrument (ROLE)" In Preskill, H. and Russ-Eft, D. Evaluation in Organizations. Cambridge, Perseus Publishing. p. 421-434.

Preskill, H. and Torres, R. (1999) Evaluative Inquiry for Learning in Organizations. Thousand Oaks, CA: Sage.

Preskill, H. and Coghlan, A. (2003). "An Overview of Appreciative Inquiry in Evaluation” In H. Preskill and A. Coghlan (eds.) Using Appreciate Inquiry in Evaluation. New Directions for Evaluation, no. 100. San Francisco: Jossey-Bass, 2003 (5-22).

Putnam, R. (1993) “The Prosperous Community Social Capital and Public Life” The American Prospect. Vol 4, no. 13, March 21, 1993. http://www.prospect.org/print/V4/13/putnam-r.html

Putnam, R. (2000). Bowling Alone: Collapse and Revival of American Community. New York: Simon and Schuster.

Raelin, J. (2000) Work-Based Learning: The New Frontier of Management Development. New Jersey: Prentice Hall.

Rossman, G. and Rallis, S. (2000). "Critical Inquiry and Use as Action”. In V. Caracelli and H. Preskill (eds.) The Expanding Scope of Evaluation Use. New Directions for Evaluation, no. 88. San Francisco: Jossey-Bass, 2000 (55-70).

Reardon, C. (2004). "Civil Society: Israel's Third Sector Comes of Age”. Ford Foundation Report. Winter, 2004. http://www.fordfound.org/publications/ff_report/view_ff_report_detail.cfm

Sanders, J. (2002). "Presidential Address: On Mainstreaming Evaluation.” The American Journal of Evaluation. (23) (3) 253-261.

Sanders, J. (2003). "The Mainstreaming of Evaluation.” In J.J. Barnette and J. Sanders (eds.) The Mainstreaming of Evaluation. New Directions for Evaluation, no. 99. San Francisco: Jossey-Bass, $2003(3-6)$.

Schein, E. (1992). Organizational Culture and Leadership. $2^{\text {nd }}$ Edition. San Francisco: Jossey-Bass. 
Schon, D.(1983). The Reflective Practitioner. New York: Basic Books.

Schram, T. (2003). Conceptualizing Qualitative Inquiry. Upper Saddle River, N. J.: Merrill Prentice Hall

Scriven, M. (1991) "Beyond Formative and Summative Evaluation." In M.W. McLaughlin and D.C. Phillips. Evaluation and Education: At Quarter Century. Chicago: University of Chicago press. $(18-64)$

Seely Brown, J and Duguid, P. (2001). Balancing Act: How to Capture Knowledge Without Killing It" In Harvard Business Review on Organizational Learning. Boston, MA: Harvard Business School Publishing. p. 45- 59.

Senge, P. (1990). The Fifth Discipline: The art and practice of the learning organization. New York: Doubleday.

Silber, I. and Rosenhek, Z. (1999) The Historical Development of the Israeli Third Sector. Beer Sheva, Israel: Ben Gurion University of the Negev.

Sterman, J. (2001). Lecture on Systems Thinking. American Evaluation Association Conference, November, 2001; Washington, D.C.

Sussman, C. (2003). "Making Change: How to Build Adaptive Capacity." Managing the Mosaic. Winter 2003, Volume 10:4.

Sussman, C. (2004). "Building Adaptive Capacity." Paper prepared for Management Consulting Service: Boston, MA.

Sweeney, L. B. (2001). When a Butterfly Sneezes. Waltham, MA: Pegasus

Torres, R. T., Preskill, H. S., and Piontek, M. E. (1996). Evaluation Strategies for Communicating and Reporting: Enhancing Learning Organizations. Thousand Oaks, CA.: Sage Publications.

Volpe, M. and Marsick, V. The nature of and Need for Informal Learning. In Informal Learning on the Job Eds. Volpe, M. and Marsick, V. Advances in Developing Human Resource, 3. 1999 p. 1-9.

Watkins \& Marsick (1999). Dimensions of the Learning Organization Questionnaire (DLOQ). http://www.partnersforlearning.com/instructions.html

Wenger, E, McDermott, R. and Snyder, W. (2002). Cultivating Communities of Practice.

Wenger, E. and Snyder, W. (2001) "Communities of Practice: The Organizational Frontier". In Harvard Business Review on Organizational Learning. Boston, MA: Harvard Business School Publishing. 1-20.

Wolf, T. (1999). Managing a Nonprofit Organization in the Twenty-First Century. New York: Simon and Schuster. 
Zeidan, E. and Ghanem, A. (2000). Patterns of Giving and Volunteering of the Palestinian Arab Population in Israel. Ben Gurion University, Beer Sheva, Israel: Israeli Center for Third Sector Research.

Ziegler, M. “Awakening”: Developing learning Capacity in a Small Family Business. In Informal Learning on the Job Eds. Volpe, M. and Marsick, V. Advances in Developing Human Resource, 3. 1999 P. 52-65. 